\title{
Rapidly Accelerated Synchronous Generators in \\ CAES Systems for Frequency Support in Power Grids
}

by

\section{Efim Sturov}

\author{
A thesis \\ submitted to the Victoria University of Wellington \\ in fulfilment of the \\ requirements for the degree of \\ Doctor of Philosophy \\ in Engineering.
}

Victoria University of Wellington 



\begin{abstract}
Modern electrical networks are transformed through the use of intermittent sources of energy, such as small-scale photovoltaic installations and wind turbines. By reducing the carbon footprints associated with centralised power grids, they are made more vulnerable to contingent under-frequency events. The renewable energy sources can't provide the required rotational inertia to make the power grid's frequency stable and to be able to assist in restoring the frequency. In New Zealand, Transpower (system operator) is responsible for normalising the frequency in case of contingent events to avoid blackouts in the networks.

In case of contingent events in power grids, additional power must be delivered to the networks with the use of primary frequency support systems. Internationally these systems are represented by under loaded power plants, where power output can be adjusted by controlling the primary governor output. This approach incurs no-load running costs and to avoid these costs generation units should be maintained at rest. The most efficient and technically feasible solution is to use synchronous generators that are already present in the power grids or can be additionally delivered to the grids as stand-alone units. However, with the use of the traditional synchronisation method, the generators cannot be synchronised with power grids in a short timeframe (up to $10 \mathrm{~s}$ in some countries).

To overcome this disadvantage, a novel synchronisation approach should be designed to synchronise synchronous generators from rest of the electrical networks. This thesis proves that it can be achieved by a ballistic synchronisation approach (and then the improved 2-stage ballistic approach), which computes and follows an acceleration trajectory which simultaneously synchronises both phase and frequency. To achieve this fast acceleration a novel environmentally friendly small-scale compressed air energy storage (ss-CAES) system has been designed. This system utilises a hydraulic drivetrain which transmits very high torque directly to the shaft of a synchronous generator, thus enabling its rapid acceleration.

The hydraulic drivetrain is composed of a proportional throttle valve and a variable-displacement hydraulic motor. The central controller from National Instruments outputs a voltage that controls the opening of the proportional
\end{abstract}


valve. It changes the flowrate in the main hydraulic circuit, meaning that it is possible to control the output torque and velocity of the hydraulic motor. Since it is coupled to a synchronous generator, the control system can control the dynamics of the drivetrain by changing its voltage output.

Computer simulations indicate that this approach enables very rapid synchronisation of a model system to the grid in $<1.5 \mathrm{~s}$ at a $100-\mathrm{kW}$ scale. The modelling of the prototype helped to verify the control parameters of the system before the implementation of the algorithm built into the hardware. It should be noted that this model was simulated with the use of the corresponding manufacturer's data. To increase the accuracy of the mathematical model and verify the control parameters, the system components were experimentally characterised with the use of a ubiquitous high-speed data acquisition system.

It resulted in a realistic and accurate mathematical model of the complex electro-hydraulic system, despite the well-known challenges of modelling the hydraulic domain. This model was utilised for the tuning of the control parameters of the system before its experimental testing. Experimental runs confirmed the feasibility of the proposed acceleration and synchronisation approach for synchronisation from the rest of the generator in $<4 \mathrm{~s}$. 


\section{Papers Published From This Thesis}

1. Efim Sturov, Chris W. Bumby, Ramesh Rayudu and Rodney A. Badcock, "Rapid synchronisation procedure for a pneumo-hydraulically driven synchronous generator," 2017 IEEE Innovative Smart Grid Technologies Asia (ISGT-Asia), Auckland, New Zealand, 2017. doi: 10.1109/ISGTAsia.2017.8378373

2. Efim Sturov, Chris W. Bumby, Ramesh Rayudu and Rodney A. Badcock, "Influence of fluid selection on synchronous generators power output in compressed air energy storage systems," 2017 IEEE Innovative Smart Grid Technologies - Asia (ISGT-Asia), Auckland, New Zealand, 2017. doi: 10.1109/ISGT-Asia.2017.8378471

3. Efim Sturov, Ramesh Rayudu, Rodney A. Badcock, Yi Yu (Patrick) Chen, Alan R. Wood and Chris W. Bumby, "Rapid synchronization procedure for a synchronous generator employing ballistic trajectory control," 2016 IEEE Innovative Smart Grid Technologies - Asia (ISGT-Asia), Melbourne, VIC, 2016. doi: 10.1109/ISGT-Asia.2016.7796438

4. Efim Sturov, Chris W. Bumby, Ramesh Rayudu and Rodney A. Badcock, "Performance and stability analysis of hydraulically driven synchronous generators in power engineering," 2018 IEEE International Conference on Power System Technology (POWERCON), Guanzhou, China, 2018. doi: 10.1109/POWERCON.2018.8602006

5. Efim Sturov, Chris W. Bumby, Ramesh Rayudu, Rodney A. Badcock and Alan R. Wood, "Demonstrated rapid ballistic synchronisation from rest of a hydraulically-driven synchronous generator," IEEE Access, DOI:10.1109 / ACCESS.2018.2883110

6. Efim Sturov, Ramesh Rayudu and Chris W. Bumby, "Modelling and experimental validation of electro-hydraulic drivetrain for synchronous generators in power engineering," ISA Transactions (under review) 


\section{Acknowledgments}

In the beginning, I want to thank my supervisors Dr Ramesh Rayudu and Dr Chris W. Bumby for their guidance, help and valuable discussions. Their knowledge, motivation and extreme patience have given me the power to excel in the research writing.

Apart from my supervisors, I won't forget to express my gratitude to Dr Rodney A. Badcock for giving encouragement and sharing insightful suggestions. Special thanks go to Gennady Sidorov for continuous discussions and engineering assistance. Also, I would like to thank the Victoria Doctoral Scholarship for financial support, which has allowed me to devote time to my research.

I am also pleased to say thank you to Peter Jannings, Derek Shotbolt, Murray Pierce and the whole Engineering and Management team at EHL Group (New Plymouth, New Zealand). They allowed me to access their research facilities and gave an opportunity to become part of their team as a visiting researcher. It wouldn't have been possible to conduct this research without their precious support.

I want to thank the entire Robinson Research Institute team. I also want to thank Joseph Bailey and Mike Davies for technical assistance, and finally Jeannie Redman and Ursula Muavae for all the administrative work, that always frees up our time to do research.

In the end, I am grateful to my family, friends and colleagues for their amazing support, encouragement and motivation over the last three years, which helped me to pursue my doctoral degree. I owe special thanks to a very special person, my wife for her continued love, support and understanding during my pursuit of the PhD that made the completion of this journey possible. 


\section{Contents}

$\begin{array}{lll}1 & \text { Introduction } & 1\end{array}$

1.1 Motivation . . . . . . . . . . . . . . . 2

1.1.1 Concept modular ss-CAES system for FIR frequency support ....................... 3

1.2 Approach . . . . . . . . . . . . . . . . 6

1.3 Research Statement and Objectives . . . . . . . . . . . . . 7

1.4 Previous work on the project . . . . . . . . . . . . 9 9

1.4 .1 Progress compared to the previous work . . . . . . . . 11

1.5 Structure of the thesis . . . . . . . . . . . . . . . . . . 12

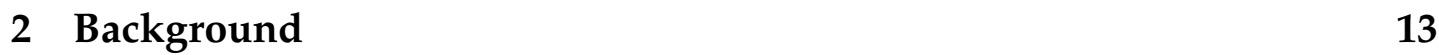

2.1 Grid Energy Storage Systems . . . . . . . . . . . . . . . . . . 13

$2.1 .1 \quad$ Pumped Hydro Energy Storage Systems . . . . . . . . . 14

2.1 .2 Electrochemical energy storage systems . . . . . . . . . . 15

2.1 .3 Flywheels . . . . . . . . . . . . . . . . 16

2.1 .4 Other Energy Storage Systems . . . . . . . . . . . . . . . . . . . . . . . . 16

2.1 .5 Compressed Air Energy Storage Systems . . . . . . . . . 17

2.2 Synchronisation Philosophies . . . . . . . . . . . . . 20

2.3 Mathematical Modelling of Hydraulic Systems . . . . . . . . . . 21

2.3 .1 Generic simulation models . . . . . . . . . . . . 22

2.3.2 Mathematical Modelling of Hydraulic Drivetrains . . . . 23

2.3 .3 Modelling of electrical components . . . . . . . . . . 26

2.4 Controller Encoder Applications in Hydraulic Drivetrains . . . 27

2.5 Chapter Summary . . . . . . . . . . . . . . . . 28

$\begin{array}{lll}3 & \text { ss-CAES System Synchronisation } & 29\end{array}$

3.1 Synchronisation Approach . . . . . . . . . . . . . . . . 30

3.1.1 Mathematical model of the proposed system . . . . . . 36

3.2 Preliminary Simulation of Synchronisation . . . . . . . . . . . 39

3.2.1 Original Ballsitic Synchronisation Approach . . . . . . . 39

3.2.2 Improved (2-stage) Ballistic Synchronisation approach . 42 
3.3 Chapter Summary . . . . . . . . . . . . . . . . . . . . . . . . . . . 43

4 Hardware for ss-CAES Prototype Experimentation 45

4.1 Hardware for Experimental Testing of the prototype . . . . . . . 45

4.1 .1 DAQ system . . . . . . . . . . . . . . . . . . . . 46

4.1 .2 Low voltage controller cabinet $\ldots \ldots \ldots \ldots \ldots$

$4.1 .3 \quad$ Electro-hydraulic drivetrain . . . . . . . . . . . . . 56

4.1.4 Mechanical limitations of the designed drivetrain . . . . 65

4.1 .5 Design of the hydraulic drum brakes . . . . . . . . 70

$4.1 .6 \quad$ Heat sink thermal analysis $\ldots \ldots \ldots \ldots$. . . . . . 72

4.2 Chapter Summary . . . . . . . . . . . . . . . . . . . . 74

5 Hydraulic valve characterisation $\quad 75$

5.1 Characterisation of the valve control stage . . . . . . . . 75

5.2 Characterisation of the valve power stage $\ldots \ldots \ldots \ldots$. . . . 81

$5.2 .1 \quad$ Hysteresis effect in hydraulic valves . . . . . . . . . . 83

5.2 .2 Hydraulic effects in the hydraulic circuit . . . . . . . . 88

5.2 .3 Hydraulic nonlinearities in the drive train . . . . . . . 89

5.3 Chapter Summary . . . . . . . . . . . . . . . . . . . . 94

6 Hydraulic motor parameterisation $\quad 95$

6.1 Signal processing and instrumentation . . . . . . . . . . . 95

6.2 Drive train acceleration measurement . . . . . . . . . . . . 98

6.3 Inertia calculation of the drivetrain . . . . . . . . . . . 102

6.4 Chapter Summary. . . . . . . . . . . . . . . . . . . . . . . . 104

7 Demonstration and Evaluation of the Synchronisation Approach $\quad 105$

7.1 Calibration of the electrical components . . . . . . . . . . 106

$7.1 .1 \quad$ Sensitivity analysis of the control system . . . . . . . . 107

7.2 Tuning of the hardware controller. . . . . . . . . . . . . . . 109

7.3 Demonstrated slow synchronisation scenario . . . . . . . . . . 115

7.3.1 Comparison of the experimental and simulated results . 117

7.4 Modelled fast synchronisation scenario . . . . . . . . . . . 120

7.4.1 Operation of the prototype at different frequency droop rates . . . . . . . . . . . . . . . . . 122

7.4.2 Robustness of the designed control system . . . . . . . . 124

$7.4 .3 \quad$ Stability analysis of the system employing the mathematical model . . . . . . . . . . . . . . . . . . 126

7.4.4 Influence of the temperature and viscosity of the fluid on the synchronisation process $\ldots \ldots \ldots \ldots 131$ 
7.5 Chapter Summary . . . . . . . . . . . . . . . . 136

8 Conclusions 139

$8.1 \quad$ Future Work . . . . . . . . . . . . . . . . . . . . . . . . . 142

8.1.1 Health and safety concerns and improvements . . . . . 144 


\section{Chapter 1}

\section{Introduction}

Renewable energy sources have the potential to reduce carbon footprints; however, they increase the pressure on the frequency of power networks, due to their intermittent nature. It has the most noticeable effect during contingent (under-frequency) events in electric networks since the renewable generation units do not create the required inertia for grid stability [1]. From this perspective, energy storage systems are the most cost-efficient way to improve this situation. This study investigates the use of rapidly accelerated synchronous generation units driven by hydraulic drivetrains to reduce the effects of intermittency in power grids, by providing a short duration - high power discharge into the electrical network for its frequency recovery. A novel acceleration and synchronisation control strategy is developed to reduce the synchronisation time of synchronous generators, forming a unique energy storage system - an ss-CAES system.

Existing power systems are facing serious transformations to deliver sustainable energy in the most environmentally friendly manner. It is mainly done with the use of intermittent sources of energy, such as solar panels (PV) and wind turbines [2, 3]. Inevitably it affects the currently installed grids in terms of components and most importantly stability. The main disadvantage of modern generation units is that they make power grids more vulnerable to frequency instabilities (due to their low inertia [1]). It can lead to a quick cascade failure (blackout) during an under-frequency event in the network. Power companies are responsible for frequency restoration in power grids and have to provide additional power to them [4]. In New Zealand, Transpower (system operator) is responsible for the use of Instantaneous Reserves (IR) to provide frequency support [5].

Modern IR systems incur idle costs since they operate all the time, and mainly not under the most optimum conditions [6]. In order to make these systems more efficient and cost-effective, it is required to find the best solution 
for IR systems that are at rest until called. Depending on the country, the time requirements for these systems are different, but in New Zealand, IR systems should start up within 1-6 s [7].

Another option to restore the frequency of the grid is the use of Interruptible Load systems (IL). Frequency stability in the grid means that demand is equal to generation. IR systems are employed to increase generation, whereas IL are used to decrease demand. Fast response IL systems can be disconnected within 1s from the grid, to initialise its frequency recovery.

To increase generation and restore the frequency, a possible solution for electrical engineers and power companies is the use of synchronous generators that can be delivered to a power grid, or that are already in the system. In this case, the primary challenge is their start-up and synchronisation time. With the use of the conventional drivetrains and synchronisation approach, their start-up time will exceed the time requirements for IRs [8]. Thus this gap in the existing generation systems can be investigated further.

\subsection{Motivation}

In New Zealand, the power frequency is equal to $50 \mathrm{~Hz}$ with a $\pm 0.2 \mathrm{~Hz}$ band, which plummets below the lower boundary in case of a contingent event [9] (Fig. 1.1). At this moment, the System Operator (Transpower) calls upon IR to restore generation and equalise it with a load. The synchronous torque drives the power grid frequency back to $50 \mathrm{~Hz}$ level while the governor control prevents frequency overshoot [10]. This approach requires the generation unit to operate below its maximum capacity, hence geothermal, solar, wind, or tidal sources could not be used as spinning reserves.

Transpower New Zealand employs two types of IR in order to respond to contingent events in the network [11]. The first one is Fast Instantaneous Reserves (FIR) which should start up within $6 \mathrm{~s}$ and generate power for $60 \mathrm{~s}$, while the second one is Sustainable Instantaneous Reserves (SIR) which should start up within $60 \mathrm{~s}$ and be able to maintain their power supply for up to 15 mins. Not every existing approach for IR is suitable for Interruptible Load (IL) application since their response times vary from $\sim 20 \mathrm{~s}$ (pumped hydro systems) to $\sim 10 \mathrm{~min}$ (gas-turbines systems) [12].

It is well known that the overall stability of a power grid containing renewable energy sources, can be improved by incorporating electrical energy storage (EES) systems within the network [13-16]. For instance, one of the technologies available in this market is Compressed Air Energy Storage (CAES). Such plants represent "mega-engineering" projects (> $100 \mathrm{MW})$, require the 


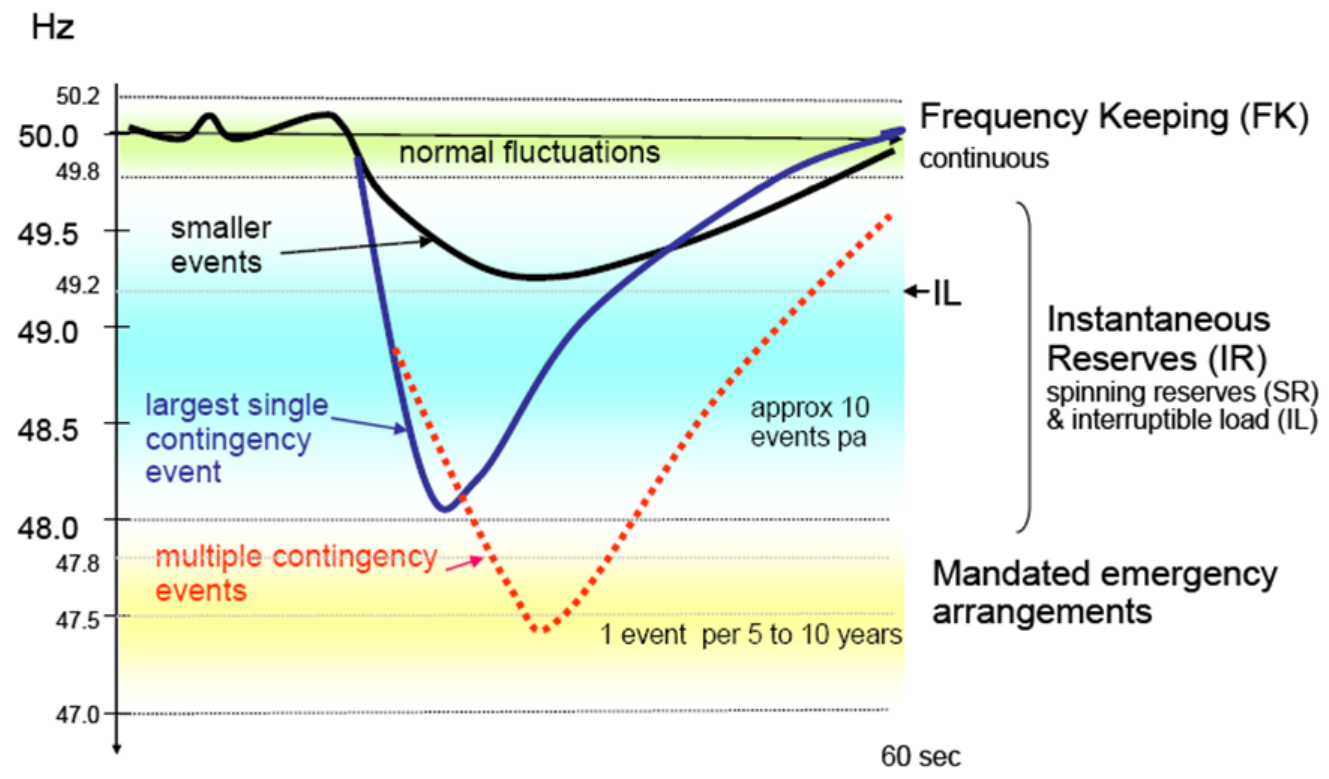

Figure 1.1: Summary of the current arrangements in NZ for frequency recovery following an under frequency event [7]

availability of vast underground caverns, and exhibit start-up times of $\sim 5-10$ mins. However, the first problem can be solved by using above-ground storage vessels, which make this approach suitable for mobile energy storage systems.

Small-scale EES technologies like supercapacitors and electrochemical storage systems have a response time of less than $1 \mathrm{~s}$. However, their installation in a power grid requires expensive power inverters [15, 17]. The alternative approach is to employ a conventional three-phase synchronous generator coupled to a high-torque fast start drivetrain, which will directly generate AC bypassing a DC-AC conversion process.

\subsubsection{Concept modular ss-CAES system for FIR frequency sup- port}

In this PhD project, a novel modular ss-CAES system will be investigated as the most suitable solution for the above-described challenge. Taking into account the required rapid start-up of the system, a hydraulic motor was chosen as a prime mover for a three-phase synchronous generator. There are multiple advantages of employing a hydraulic motor-pump, such as its availability "off-the-shelf", very high power density ( $1 \mathrm{MW}$ per litre), high conversion efficiency $(>92 \%)$, rapid start-up $(<0.1 \mathrm{~s})$, long service lifetime ( $>10$ years), and low cost $(\mathrm{NZ} \$ 15 \mathrm{k}$ per MW). The prototype was built in cooperation with EHL Group, Taranaki, New Zealand and Australia.

Fig. 1.2 depicts a block diagram of the $100 \mathrm{~kW}$ prototype ss-CAES system 
employed in this project, which employs a pneumohydraulic accumulator to store energy in the form of compressed gas. When triggered, this stored energy is released, and pressure drives fluid through the hydraulic circuit. This accelerates the hydraulic motor which is directly coupled to the shaft of a 4pole 3-phase synchronous generator. The acceleration profile is controlled using a hydraulic proportional throttle valve (Parker TDA016). This is a poppet valve, in which the orifice size is controlled by the solenoid drive current. Such proportional valves are relatively low cost but do not provide servo-control of the orifice size. Instead, our approach employs feedback control based on the generator's phase, speed, and acceleration, which are measured using a quadrature incremental position encoder installed on the shaft of the electrical machine. A three dimensional model of the prototype system is shown in Fig. 1.3 .

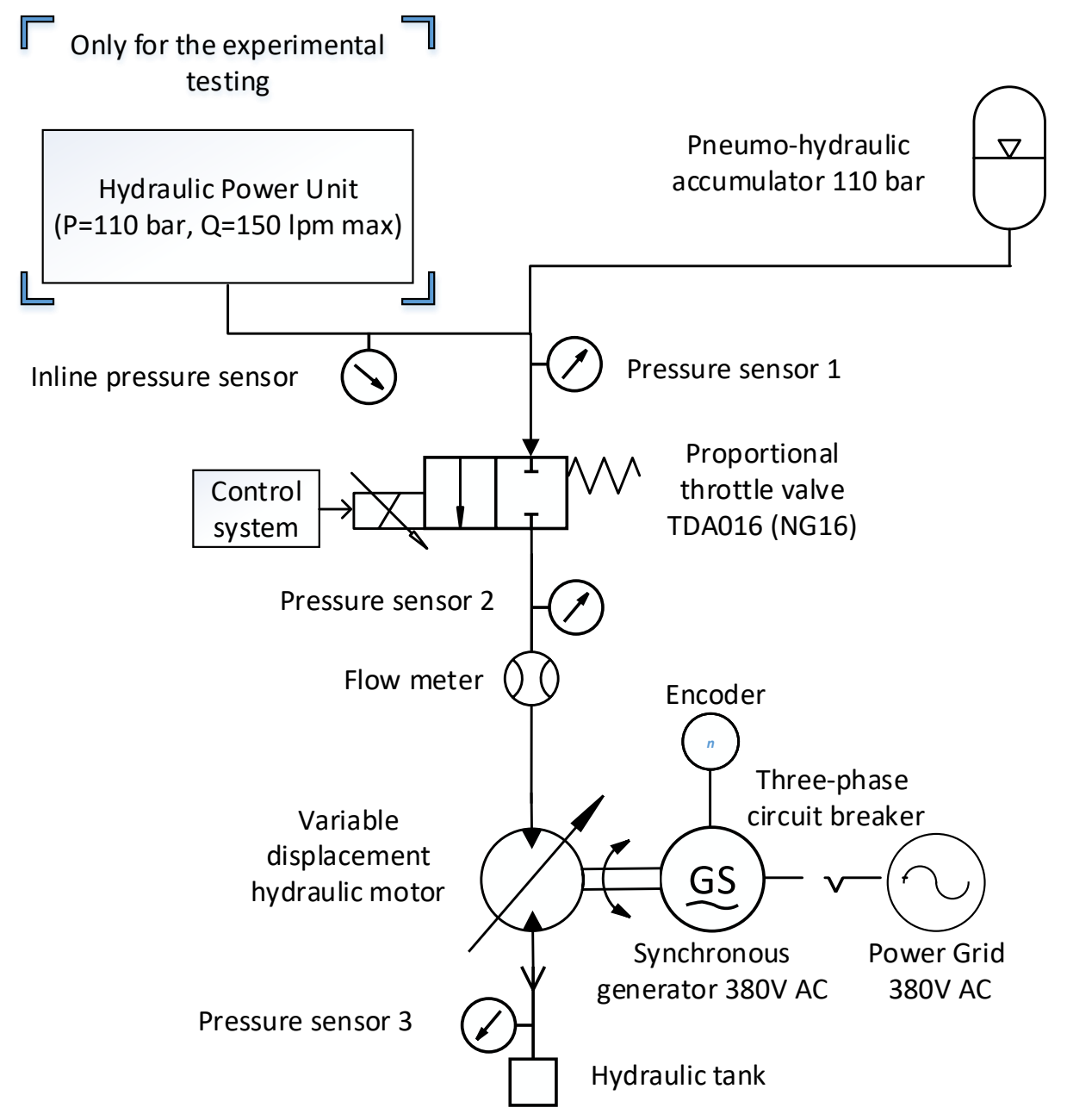

Figure 1.2: Schematic of the ss-CAES prototype.

The power capacity of this system is defined only by the sizing of the hydraulic motor and generator, which is different from other Energy Storage sys- 


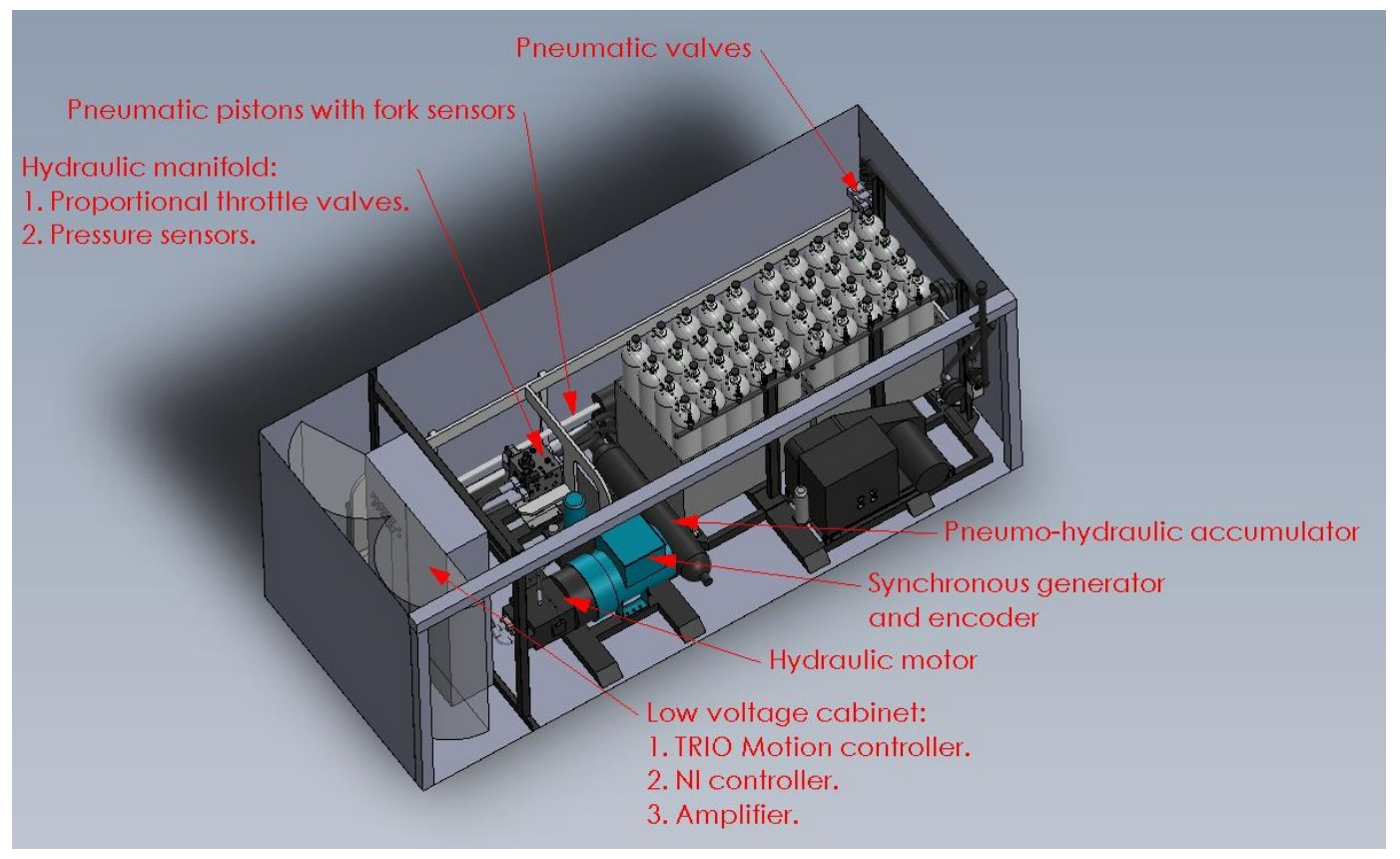

Figure 1.3: A three dimensional model of the ss-CAES prototype.

tems (ESSs). These single components represent a small fraction of total system cost, so high power short time discharge systems can be implemented at a significantly lower cost than parallel banks of batteries, super-capacitors, or flywheel approaches. Very high pressures can be achieved in small pneumohydraulic accumulator vessels, which leads to very high power density [18]. Hydraulic accumulators store energy by using a "liquid piston" to compress a volume of gas [19].

The original idea was to design and build a 1MW CAES system. This system will be significantly larger and more expensive than the presented $100 \mathrm{~kW}$ prototype. However, some differences between these two systems exist. First of all, a 1MW generator might not be financially and technically feasible to employ in the system, but rather two $500 \mathrm{~kW}$ generators is a possible solution. In terms of the powertrain, it will require to use four $250 \mathrm{~kW}$ hydraulic motors and gearboxes to transmit their torque to the generators. The amount of stored air (gas) will also determine the size of the system, which will be a plant size level, rather than being a mobile transportable solution. Considering the cost implications, a decision was made to built a $100 \mathrm{~kW}$ prototype to verify the main principles of its operation.

To reduce the synchronisation time of the proposed electro-hydraulic drivetrains with synchronous generators, it is necessary to develop a new synchronisation approach. The first step was made with the method called the ballistic synchronisation approach [20, 21] by Dr Alan Wood and an MSc student Patrick Chen from the University of Canterbury. However, the proposed 


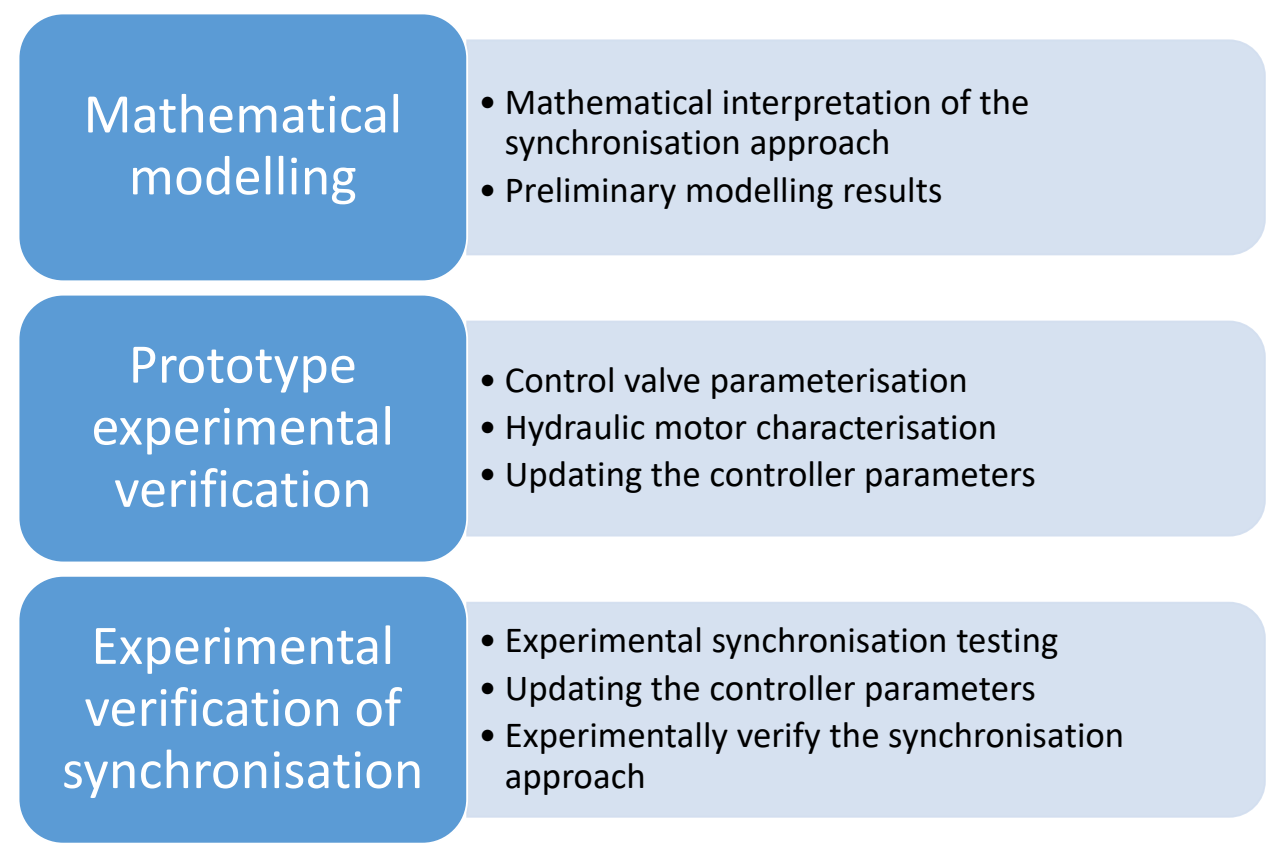

Figure 1.4: Testing approach for the verification of the synchronisation approach by employing the ss-CAES prototype.

method could not synchronise the synchronous generation unit with the power grid, since the synchronisation windows were too short $(5-8 \mathrm{~ms})$. The current research focuses on the improved ballistic method (also called the quasiballistic or 2-stage ballistic approach) and will be further verified in the presented work.

\subsection{Approach}

Since there are two main components of the proposed frequency support system, it is necessary to study both the electro-hydraulic drivetrain and the synchronisation approach. The approach for the testing of the system is summarised and presented in Fig. 1.4 .

The approach verification starts with its mathematical modelling. The model at this stage is based on the written acceleration and synchronisation algorithm and also includes the electro-hydraulic drivetrain for the synchronous generator. All components in the model are based on the available manufacturer's data. It makes it possible to mainly verify the feasibility of the derived mathematical approach, rather than any control parameters of the system.

The next step in the experimental verification of the approach is drivetrain characterisation. It starts with the experimental verification of the valve (described in detail in Chapter 5), which took place in New Plymouth, Taranaki, New Zealand. It was followed by updating the mathematical model of the pro- 
totype in order to estimate the dynamics of the motor before its experimental testing.

The motor's parameterisation took place at the EHL site in New Plymouth, New Zealand (see Chapter 6). It is followed by the next stage of updating the mathematical model to tune the central controller's parameters to perform the next synchronisation testing.

The first synchronisation test was done with the use of the logged voltage profiles from the mathematical model that were uploaded to the central hardware controller. During this testing, there was no feedback fed from the electro-hydraulic drive to the control system. These tests were performed to verify the accuracy of the controller's parameters.

The final testing of the synchronisation approach was performed with the full control loop, including dynamic feedback from the generator to the control system.

The system's parameterisation comprises two steps - preliminary modelling and its experimental verification. With a newly obtained experimental dataset, it is required to update the model and rerun the simulations in order to predict the behaviour of the next tested component. With the continually updated mathematical model, it is possible to update the controller parameters of the system and utilise them later in the hardware built.

\subsection{Research Statement and Objectives}

The goal is to develop a cost-effective, environmentally friendly energy storage system at $100 \mathrm{~kW}-1 \mathrm{MW}$ scale that can provide frequency support during contingent events in a grid. The presented system is an ss-CAES system which employs a hydraulically driven synchronous generator. So far the primary application for an ss-CAES system is an energy storage system for remote areas, which is quite often combined with small wind or solar farms. These systems are not employed for frequency support in power grids due to their long synchronisation time ( $>6 \mathrm{~s}$ for a 100-kW system); hence it is the novel and promising application of this type of systems. In order to synchronise the proposed device with a power grid, a novel acceleration technique will be utilised. It is based on the original ballistic synchronisation approach modified to provide long enough synchronisation windows for a generator. This system will provide high power - short duration discharge, with a response time $<1 \mathrm{~s}$ (6 s as an FIR system) and maintain its power supply for $1 \mathrm{~min}$ (FIR) or up to $15 \mathrm{mins}$ (SIR).

The design of hardware elements and control algorithms for such systems 
requires the comprehensive initial modelling and analysis of a combination of electrical, control, hydraulic, and pneumatic systems. The conventional approach for the design of an ss-CAES system is the calculation of their energy capacity and simple electrical modelling of a DC-AC conversion process. This thesis highlights a unique approach for modelling and design of a pneumohydraulic system which drives an electrical system. Furthermore, it starts from the described isolated system (energy storage), and connects it to a power grid, when this system becomes a generation unit for frequency support of the network.

The biggest challenge is to invent a control approach that can rapidly accelerate and connect the system to a power grid in less than $6 \mathrm{~s}$.

Our proposed acceleration technique fulfils the requirements for synchronisation with a power grid (phase angle and frequency of the generator). The control strategy is verified by the use of a computer model, followed by the experimental validation of a $100 \mathrm{~kW}$ hardware prototype. The system is built in collaboration with our industrial partner, EHL Group (Taranaki) [22].

To achieve this research statement, the following set of research objectives was studied:

1. The proposed synchronisation approach (improved ballistic trajectory/ 2-stage ballistic approach) of the ss-CAES prototype should be verified with the use of simulation tools. The model should be able to simulate all transients in the system, and this data should ensure the safe operation of the prototype before and during its experimental testing.

Tasks: Considering the proposed prototype, its cost, and potential danger, related to non-reliable control techniques or mistakes in the design, a reliable mathematical model should be developed for analysis. Since this prototype combines different fields of electrical, automation control and mechanical engineering, the most widely used multi-domain software MATLAB Simulink is utilised for the simulation studies.

Outcome: The project will investigate different approaches to the modelling of a multi-domain system with an interconnection between various fields of study. The outcome is a comprehensive mathematical model that is extensively tested and verified.

2. To improve the accuracy of the designed mathematical model and make it more realistic, it is required to characterise the main components of the hardware prototype experimentally. This can be done with the use of the data acquisition system built within the experimental setup. To achieve 
the most reliable and feasible results, step-by-step (e.g., component-bycomponent) experimental verification is needed.

This method significantly increases the overall accuracy of the engineered mathematical model and creates an opportunity to simulate and study in depth every characterised device or component.

Tasks: A ubiquitous data acquisition system should be developed to ensure that the main components of the system can be verified in a time efficient manner with the required accuracy.

The designed mathematical model should be modified in order to either utilise the experimental data directly or accurately mimic them.

Outcome: This study proposes a data acquisition system that leads to the design of an accurate and realistic mathematical model of the complex electro-hydraulic prototype.

3. To implement, in hardware, the proposed 2-stage ballistic acceleration approach (by utilising a high-speed industrial controller), so the prototype can successfully start up and accelerate from rest in less than $6 \mathrm{~s}$.

Such testing can be performed after the characterisation process is completed and the controller in the mathematical model is tuned for stable synchronisation. This process also increases the overall safety of the experimental runs by knowing the system stability boundaries and estimated controller parameters.

Tasks: It can be achieved via the programming, testing, and troubleshooting of a 2-stage ballistic synchronisation algorithm that will be uploaded to the central high-speed controller.

Outcome: This study focuses on the programming of the employed central controller and the extensive testing of the main components of the prototype, to ensure its robust operation.

\subsection{Previous work on the project}

The original ballistic mathematical approach was designed by Dr Alan Wood from the University of Canterbury. The experimental part of the project was done by an MSc student from the University of Canterbury - Yi-Yu (Patrick) Chen [20, 21].

The first version of the mathematical model was designed at the UoC and employed the ballistic method. The model comprised a few main components, 
which were either removed or modified during the last three years of the current research.

- The Power network is modelled as a three-phase voltage source. This part of the model has been changed during the current research. Remembering that the current experimental testing is focused on the proposed synchronisation approach, it is possible to use only the frequency and phase outputs from this subsystem.

- The Phase Locked loop system was fully described in Chen's MSc Thesis [20]. Since there is no actual power grid employed for the experimental testing, the PLL system is not included in the final version of the control system.

- The Acceleration Derivation system. This mathematical block includes the main acceleration algorithm that was initially designed at the UoC. During the presented research, this script has been modified in order to improve the ballistic approach and make it possible to run the 2-stage ballistic algorithm. Also, different control parameters have been changed in order to control the synchronisation time and acceleration/phase profiles of the generator.

- The Controller. This system is used for the calculation of the control voltage that has to be sent to the proportional throttle valve (TDA016) in order to make the generator follow a computed acceleration trajectory. The main components of the controller are a feedforward controller and a PIcontroller. These subsystems have been changed a few times throughout the testing and modelling of the prototype. The final values of the controller parameters lead to the system's stable synchronisation.

- The Hydraulic drivetrain. This part of the mathematical model simulates the real hardware drivetrain. The main components of it are a proportional throttle valve (TDA016) and a variable displacement hydraulic motor M14. The main hydraulic circuit was assembled in the prototype a few years ago; but this hardware was neither experimentally characterised nor accurately modelled. The original mathematical mode designed at the UoC was based on the manufacturer's data of the main components, thus was not accurate for the control system's tuning. It should be noted that the model utilised a wrong size proportional valve (TDA032). 


\subsubsection{Progress compared to the previous work}

To highlight the achieved progress during this $\mathrm{PhD}$ it is beneficial to compare what was achieved before the start of this $\mathrm{PhD}$ program with the progress during it.

- Synchronisation approach. Chen et al.'s [20] mathematical model showed that the proposed synchronisation approach could accelerate and synchronise a synchronous generator with a power grid. However, the presented results in [20, 21] show that this method is not capable of achieving long enough synchronisation time windows to close a circuit breaker to connect a generator to the grid (time response $>50 \mathrm{~ms}$ ). They report that the maximum synchronisation time window is around $8-9 \mathrm{~ms}$, which is not long enough for mechanical circuit breakers to operate. The research presented in this thesis improves the synchronisation approach by extending the synchronisation windows up to $500-800 \mathrm{~ms}$, which is long enough for any circuit breakers to trigger and connect a generator to the grid.

- Improved data acquisition system. Chen et al. [20] experimentally verified the main control hydraulic valve with the use of a test rig (not the real ss-CAES system hardware setup). The setup employed in that data acquisition system was designed around TRIO motion controllers, making it challenging and time-consuming to use the obtained data in the main controller from National Instruments that is utilised in the real ss-CAES system hardware prototype. This thesis presents experimental data obtained from a data acquisition system within the real prototype. The logging of all channels and outputs through one high-speed controller made the data analysis process fast and reliable.

- Component-based verification and refinement of the mathematical model. The mathematical model from [20] was based on the manufacturer's data and some experimental data acquired through the test rig. Step by step experimental verification showed that the same components in the prototype perform drastically differently. Only full experimental testing of the hardware setup with the constant tuning of the mathematical model and the central NI controller can give accurate and realistic results.

A summary of the progress during the work on this project is available in Table 
Table 1.1: Preliminary work on the project vs. the current progress

\begin{tabular}{|l|l|l|}
\hline & Ballistic synchronisation approach & 2-stage ballistic synchronisation approach \\
\hline Synchronisation approach & $\begin{array}{l}\text { The proposed approach does not lead } \\
\text { to the connection of a synchronous } \\
\text { generator to a power grid, due to its } \\
\text { short synchronisation windows }\end{array}$ & $\begin{array}{l}\text { The improved ballistic approach increases } \\
\text { the synchronisation windows up to 500-800 ms } \\
\text { making it possible to connect a generator } \\
\text { to a power grid }\end{array}$ \\
\hline Mathematical model & $\begin{array}{l}\text { The designed mathematical model } \\
\text { is based on the manufacturer's data } \\
\text { of the main components }\end{array}$ & $\begin{array}{l}\text { Every component of the hardware prototype was } \\
\text { experimentally characterised to create a realistic } \\
\text { mathematical model }\end{array}$ \\
\hline Hardware prototype & $\begin{array}{l}\text { The hardware prototype was not } \\
\text { ready for its experimental testing }\end{array}$ & $\begin{array}{l}\text { The assembling of the hardware prototype was } \\
\text { finished }\end{array}$ \\
\hline Data acquisition system & $\begin{array}{l}\text { DAQ system based on a TRIO motion } \\
\text { controller in a test rig, making the use } \\
\text { of the logged data limited }\end{array}$ & $\begin{array}{l}\text { A ubiquitous DAQ system based on a high-speed } \\
\text { National Instruments controller was designed }\end{array}$ \\
\hline
\end{tabular}

\subsection{Structure of the thesis}

The thesis is organised as follows:

Chapter 2 gives a literature review and presents background knowledge: existing literature regarding energy storage systems utilised electric and hydraulic components.

Chapter 3 discusses the mathematical background of the proposed synchronisation approach and the initial simulation results.

Chapter 4 presents the detailed description of the main components of the system with the corresponding data acquisition (DAQ) system.

Chapter 5 highlights the experimental characterisation process of the proportional throttle valve that is the primary control device in the discussed electro-hydraulic drivetrain.

Chapter 6 focuses on the experimental verification of the variable displacement hydraulic motor that accelerates and drives the synchronous generator for its synchronisation with the power grid.

Chapter7depicts the final experimental testing of the proposed synchronisation approach by utilising a synthetic hardware-in-the-loop system.

Chapter 8 summarises the work presented in this thesis, draws a conclusion and discusses potential, future work. 


\section{Chapter 2}

\section{Background}

In this thesis, the primary focus of the proposed system is frequency support in power grids being utilised as an energy storage system. An overview of different energy storage solutions used as spinning reserves is presented in this chapter. The chapter covers the range of different energy storage systems available in the market and the main advantages of the proposed ss-CAES system.

One of the significant contributions of this research is a novel 2-stage ballistic synchronisation approach for synchronous generators driven by an electrohydraulic drivetrain. Hence the significant trends and advances in electrical, control and hydraulic systems are discussed in the chapter. Other synchronisation methods are presented in this chapter to allow comparison to the proposed method. From the hardware implementation perspective, it is required to review the literature on data acquisition systems (National Instruments).

\subsection{Grid Energy Storage Systems}

A serious disadvantage of sustainable energy resources is their variable and intermittent nature [23]. Hence the generation units employing these sources cannot fulfil the requirements to operate as FIR systems. Overall, fluctuations in the output power of these systems can cause serious voltage sags in a power grid, which potentially can be compensated through the use of energy storage systems [24-28].

A brief analysis of the field shows that pumped-hydro systems cover the majority of energy storage capacity. However, they heavily depend on a geographical site where they are built. Moreover, due to the high inertia of these systems, they cannot be synchronised with a power grid within $1 \mathrm{~s}$. A short comparison of different energy storage systems is presented in Table 2.1] [29- 
31].

Table 2.1: Energy Storage Systems

\begin{tabular}{|c|c|c|c|c|c|}
\hline Type & $\begin{array}{l}\text { Energy } \\
\text { Effi- } \\
\text { ciency } \\
(\%)\end{array}$ & $\begin{array}{l}\text { Energy } \\
\text { Density } \\
(\mathrm{Wh} / \mathrm{kg})\end{array}$ & $\begin{array}{l}\text { Power } \\
\text { Density } \\
(\mathrm{W} / \mathrm{kg})\end{array}$ & $\begin{array}{l}\text { Cycle Life } \\
\text { (cycles) }\end{array}$ & $\begin{array}{l}\text { Self Dis- } \\
\text { charge }\end{array}$ \\
\hline Pb-Acid & $70-80$ & $20-35$ & 25 & $200-2000$ & Low \\
\hline $\mathrm{Ni}-\mathrm{Cd}$ & $60-90$ & $40-60$ & $140-180$ & $500-2000$ & Low \\
\hline $\mathrm{Ni}-\mathrm{MH}$ & $50-80$ & $60-80$ & 220 & $<3000$ & High \\
\hline Li-Ion & $70-85$ & $100-200$ & 360 & $500-2000$ & Med \\
\hline $\begin{array}{l}\text { Li- } \\
\text { polymer }\end{array}$ & 70 & 200 & $250-1000$ & $>1200$ & Med \\
\hline $\mathrm{NaS}$ & 70 & 120 & 120 & 2000 & - \\
\hline VRB & 80 & 25 & $80-150$ & $>16000$ & Negligible \\
\hline EDLC & 95 & $<50$ & 4000 & $>50000$ & Negligible \\
\hline $\begin{array}{l}\text { Pumped } \\
\text { hydro }\end{array}$ & $65-80$ & 0.3 & - & $>20$ years & Negligible \\
\hline CAES & $40-50$ & $10-30$ & - & $>20$ years & Negligible \\
\hline $\begin{array}{l}\text { Flywheel } \\
\text { (steel) }\end{array}$ & 95 & $5-30$ & 1000 & $>20000$ & Very High \\
\hline $\begin{array}{l}\text { Flywheel } \\
\text { (compos- } \\
\text { ite) }\end{array}$ & 95 & $>50$ & 5000 & $>20000$ & Very High \\
\hline
\end{tabular}

Some papers employ a Ragone plot [32] which represents the relationship between power and energy density of different energy storage systems as it is shown in Fig. 2.1] [31].

\subsubsection{Pumped Hydro Energy Storage Systems}

Pumped Hydro Energy Storage (PHS) is the most widely used and the oldest energy storage system. They employ the potential energy of the water pumped to an upper reservoir, which will be transformed into kinetic energy of the falling water in a lower reservoir (by a turbine) when the water is released during peak demand periods [28, 33]. Equation 2.1] shows that the energy stored in this system is proportional to the difference in the height between the reservoirs and the volume of the stored water [33].

$$
E_{\text {storage }} \alpha V\left(H_{\text {upper }}-H_{\text {lower }}\right)
$$




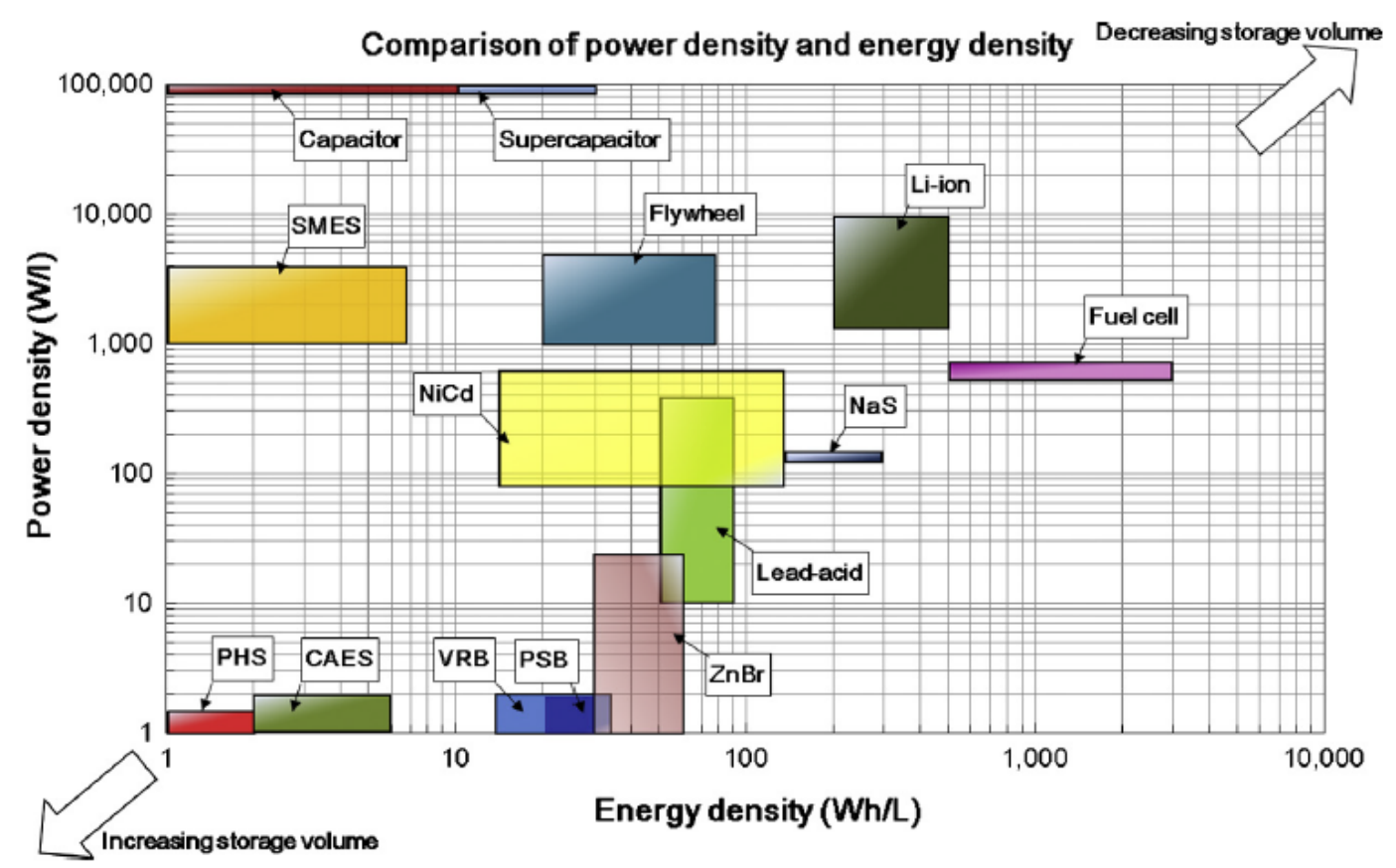

Figure 2.1: Comparison of energy density and power density [31]

where $E_{\text {storage }}$ is the generated energy $(\mathrm{kW}), \mathrm{V}$ is the volume of the stored water $\left(m^{3}\right), H_{\text {upper }}$ is the height of the upper reservoir $(\mathrm{m})$ and $H_{\text {lower }}$ is the height of the lower reservoir $(\mathrm{m})$.

The main advantages of PHS systems:

1. High efficiency of the system (around 75\%) [24].

2. Relatively low capital cost per unit of energy [34].

3. Long storage period (hours-months).

4. Long lifetime (40-60 years) [33].

Regarding the disadvantages of the system we can highlight the following:

1. The system heavily relies on a geographical site since it requires high elevation to create the height difference between the reservoirs [24].

2. Long lead time and the high cost of construction and environmental issues (removing trees from the land) [34].

\subsubsection{Electrochemical energy storage systems}

The main advantage of these systems is their fast installation, even when combined into a large battery system. The major concern of such systems is a batteries' life cycle, which depends on their Depth of Discharge (DoD). There are 
a variety of different technologies. However, some of them are not fully presented in the market, while others are expensive making them unreliable for installation [10, 24, 25, 30, 33, 34].

\subsubsection{Flywheels}

This system stores energy in the form of kinetic energy, by spinning a mass during an excess energy period [15, 24, 32, 35, 37]. During this time, a flywheel is spun by a motor, whereas during its discharge, the same motor acts as a generator, producing electricity by employing a flywheel as a prime mover. The total amount of energy stored in a flywheel depends on its size and the speed of its rotor [34]. The most significant advantage of flywheels is that they have a long life cycle and are capable of providing hundreds of thousands of full charge-discharge cycles (unlike batteries) [33], [36]. Their efficiency is also in the range of 90-95\% [34]. However, a flywheel has a very high capital cost, ranging from 1,000 to $5,000 \$$ per $\mathrm{kWh}$, and a high rate of discharge in the range of $50-100 \%$ per day [25].

\subsubsection{Other Energy Storage Systems}

There is a significant number of other technologies that are used for energy storage. However, their efficiency is either too low, or their manufacturing process is too complicated. The list includes, but is not limited to, the following options:

1. Capacitors. They store electricity at a fast rate and are suitable for stability voltage applications. Nevertheless, they have high power density but lower energy density. This downside triggered research regarding electrochemical capacitors, also known as supercapacitors [16, 38-43].

2. Superconducting magnetic energy storage. This method employs direct current flowing through a superconducting coil, so the energy is stored in the magnetic form until it is needed. The main components of the system are a superconductor, a cryogenic refrigerator, a power conversion system (PCS) and a control system. This approach has exceptional power density and an expected lifespan of more than 20 years. However short duration discharge, high cost, and environmental concerns about a strong magnetic field make this system rarely used in the industry [16, 26, 33, 37, 40, 44, 45]. 


\subsubsection{Compressed Air Energy Storage Systems}

The main idea behind this concept dates back to the 1940s when the patent application "Means for storing fluids for power generation" was submitted by F.W.Gay to the US patent office [46]. The principle of its operation is storage of compressed air that can be converted to electricity using a turbine generator [17, 27, 43, 46-54]. Short descriptions of some of the different approaches are presented in this section.

\section{Conventional or adiabatic CAES systems (D-CAES)}

The two most well-known D-CAES plants were constructed in Huntorf (Germany) and McIntosh (Alabama, USA) which store cooled air at 44-70 bar of pressure in underground caverns. During the generation phase, this air is preheated through a heat recuperator, mixed with natural gas and burned in a combustion chamber $\left(550 \mathrm{C}^{\circ}\right)$ and expanded through a multistage-coupled turbine generator [27, 34, 51, 53].

\section{Hybrid compressed air and thermal storage systems}

To move away from the use of fuel in the classic CAES system design, alternative, fuel-free CAES systems have been investigated, with the idea of storing heat generated at a compression stage that is used later to reheat high-pressure air before expansion [27, 49, 54].

\section{Advanced Adiabatic - compressed air energy storage systems (AA-CAESS)}

The basic idea of the AA-CAES concept is the use of a heat storage unit as the central element of the plant. During the charging phase, compressed air must be cooled to the temperature of $50 \mathrm{C}{ }^{\circ}$ for storage in salt caverns. At the discharge stage, this air is heated up [27, 45].

\section{Uncooled compressed air energy storage systems (U-CAESS)}

This concept represents a system where compression heat is stored together with compressed air in an isolated reservoir. However, because of the storage vessel's non-ideal insulation, the storage time is limited. Meaning that this technology is only suitable for short-time storage applications [27, 46].

\section{Hybrid thermal and compressed air energy storage systems (TACASS)} This mechanism is essentially a standalone and smaller version of the classic CAESS with the following differences [27, 39, 55]: 
(a) TACAS systems use conventional high-pressure cylinders to store the compressed air.

(b) TACAS systems use a low-cost flywheel to maintain power quality and provide a few seconds of bridging power during operation of the system [27].

\section{Isothermal compressed air energy storage systems (I-CAESS)}

These systems attempt to achieve near-isothermal compression in situ, with the improved system efficiency (70-80 \%), which provides fuel-free operation and reduces thermal stress on the utilised equipment. A few patented I-CAESS technologies are under development so far [17, 48].

\section{Small-scale compressed air energy storage systems (ss-CAESS)}

These are fully mobile systems that can provide a longer lifespan and can be integrated with existing heat and cooling sources available on a site in a cogeneration application [46].

1. Hydro-pneumatic energy storage systems.

Hydro-pneumatic systems have better heat exchange and a lower risk of leakage and friction losses in comparison to purely pneumatic systems [10, 36, 38, 39, 46]. It has led to the development of two hydro-pneumatic storage systems depicted in Fig. 2.2 that employ the acronym "BOP" Batteries with Oil-hydraulics and Pneumatics.
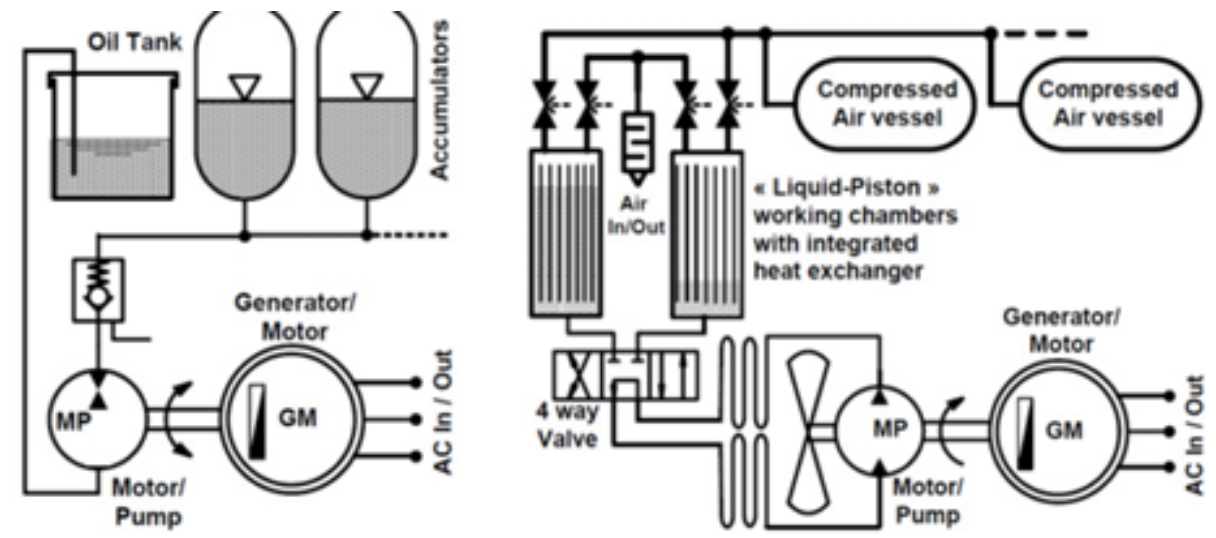

Figure 2.2: Schematic diagram of pneumatic storage systems with oilhydraulic conversion [38, 39]

The type A systems employ industrial pneumohydraulic accumulators as storage and separation devices between air and oil which helps to provide high instantaneous power release. 
Hydraulic motors have exceptional energy-efficiency performance. A combination of these systems can help to achieve high efficiency when operating in isothermal cycles. It is achieved when the charging time of an accumulator is much higher than the time-constant needed for the thermal exchange between the surrounding and the accumulator's internal chamber. The main disadvantage of these systems is their low power density due to the fixed amount of the stored gas.

The type B system is better in this respect since it uses a reduced amount of oil in a closed circuit to compress fresh air from the outside. The primary approach is to employ a converter to compress and expand air with high-efficiency to maintain almost constant temperatures during compression or expansion. However, it requires an oil-to-air "liquid piston" interface which is difficult to implement [38, 39].

Rufer and Lemofouet proposed a Maximum Efficiency Point Tracking Method for their BOP design to vary the output power, because of the continuous changes in pressure and load affecting the pneumatic machine's performance. Their approach is based on the measurements of pressure, flow rate, and speed, which ae used to calculate the optimal speed for the maximum efficiency. However, this method is not applicable to the proposed ss-CAES system, because the employed synchronous generator rotates at a constant velocity determined by the power grid frequency.

2. Compressed air with wind and wind-diesel hybrid systems.

These systems are used mainly in remote areas to improve the exploitation of diesel generators or small wind farms [44, 45]. During periods of strong wind, energy is used to compress air via a compressor and store it. Afterwards, this air is used to turbocharge a diesel engine and decrease its fuel consumption [27].

By summarising the review, we can state that the most efficient solution is the use of technology which can generate AC voltage to a power grid directly. If battery systems are used, expensive and complicated inverters should be employed for the conversion from DC to AC [26, 35, 42, 56, 57].

Potentially, flywheels could be connected to a synchronous machine, which can be used efficiently in either a motor or generator mode. However, the fast self-discharge of these systems makes them unsuitable for long-term storage systems [15]. Nevertheless, CAES systems can provide high power density, a great number of recharge cycles, and a long lifespan [33, 46, 51]. The main 
disadvantage is the same as for PHS systems - dependency on their geographical location [24, 25, 34, 36]. However, it is possible to solve this issue by using aboveground storage vessels [15, 49, 50], which could provide an opportunity to use a fuel-free mobile system as an IL for power grids. Hence they can be delivered and installed either on distribution or transmission sides or operated as emergency response units for remote areas [24, 35, 36, 45, 54].

\subsection{Synchronisation Philosophies}

The approach of employing a synchronous generator was chosen for this study as the most feasible solution for the Energy Storage System, therefore, it is necessary to review a process of its connection to a power grid [58, 59]. A research group from Russia and Taiwan published a paper focused on a synchronisation technique based on a reference model. The approach is similar to the ballistic approach, however, has some weak points which will be highlighted later. Fig. 2.3 shows a block diagram of the studied prototype.

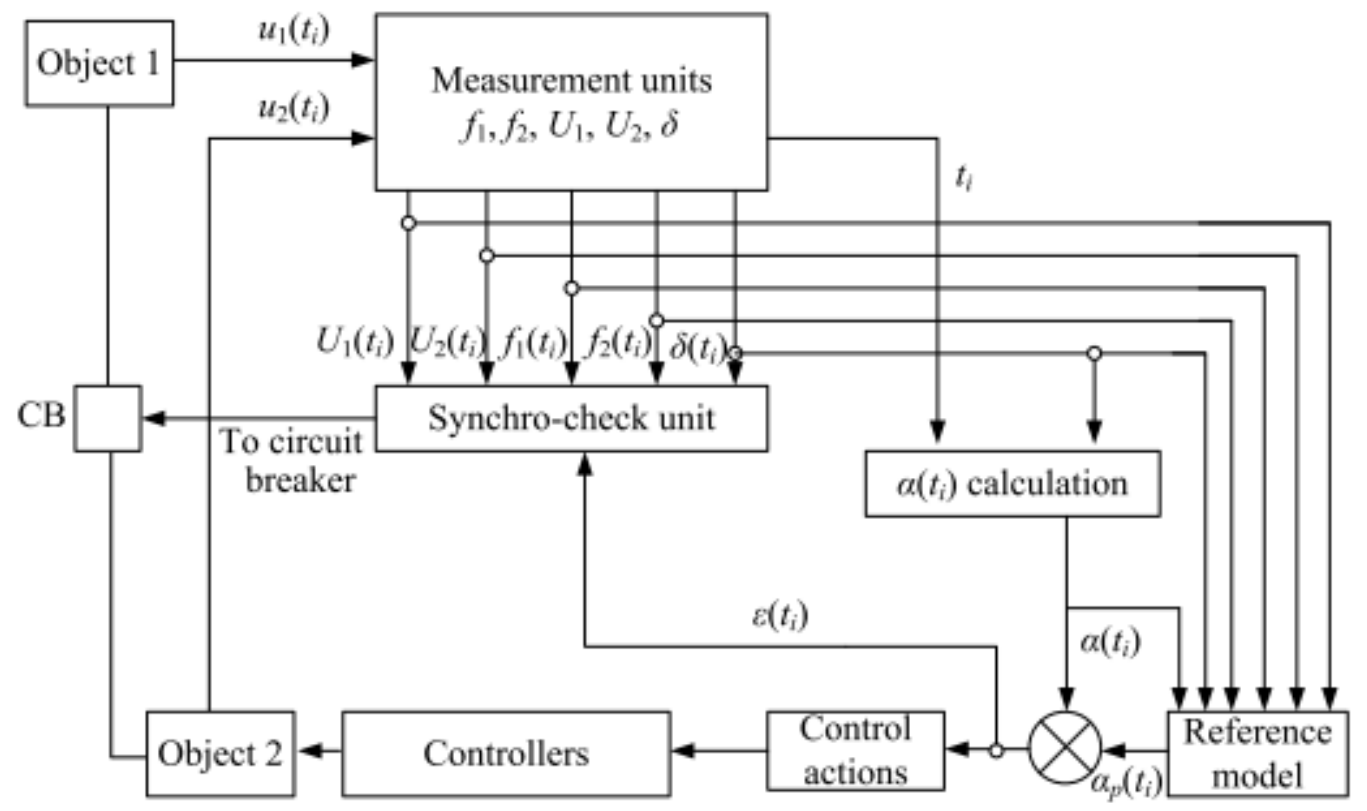

Figure 2.3: Function block diagram of adaptive synchronisation [59]

Terminal and grid voltages are fed to the Measurement Unit (MU) for the evaluation of the voltage magnitudes, frequencies, and phase angles. Then these parameters are sent to the reference model block, where an acceleration trajectory is computed. There is no information about the topology of the reference model system, whereas in the system studied in the thesis a PLL module is used as the synchronisation unit. 
One of the main disadvantages of the method presented in the paper [59] is that Automatic Voltage Regulator (AVR) operation is not reviewed "since AVR usually provides the voltage magnitude without any complications". In this case, it is worthwhile to provide excitation and terminal voltage plots as part of the described synchronisation process. The main idea behind the acceleration philosophy is to obtain the following conditions for synchronisation (equations 2.2, 2.3, 2.4):

$$
\begin{gathered}
\delta_{T}=\delta_{2 T}-\delta_{1 T}=2 \pi n \\
v_{T}=\omega_{2 T}-\omega_{1 T}=0 \\
\alpha_{T}=\alpha_{2 T}-\alpha_{1 T}=0
\end{gathered}
$$

where $\delta_{1 T}, \delta_{2 T}$ - phase angles ( $\left.\mathrm{rad}\right), \omega_{1 T}, \omega_{2 T}$ - cycling frequencies and $v_{T}$ - rotor speed ( $\mathrm{rad} / \mathrm{s}), \alpha_{1 T}, \alpha_{2 T}$ - accelerations $\left(\mathrm{rad} / \mathrm{s}^{2}\right)$ of the synchronisation objects one and two at the moment $t_{T}, \mathrm{n}$ - synchronisation objects voltage phasors relative cycle number.

After computing an acceleration value in the control block, it is sent to the reference model block where a trajectory is derived. A power imbalance trajectory that is used to provide the final synchronisation parameters at the instant of time is calculated according to 2.5 .

$$
\Delta P\left(t_{i}\right)=P_{m}-P_{e}-P_{\text {loss }}=\varepsilon\left(t_{i}\right) \frac{T_{j}}{\omega_{g}}
$$

where $P_{m}$ - generator mechanical power (pu); $P_{e}$ - generator electric power $(\mathrm{pu}) ; P_{\text {loss }}$ - power losses (pu); $T_{j}$ - inertia constant (s) and $\omega_{g}$ - generator cycling frequency $(\mathrm{rad} / \mathrm{s})$.

Damping control is not reviewed in [59] since an AVR system is not presented and according to the authors "a damping control is a duty of a power system stabiliser and is not a responsibility of the proposed technique". Simulation of a contingent event is presented; however, it shows a frequency droop from $50.03 \mathrm{~Hz}$ to $49.8 \mathrm{~Hz}$. Since it is the only presented example, it is difficult to verify the feasibility and robustness of the described approach.

\subsection{Mathematical Modelling of Hydraulic Systems}

Many hydraulic systems, actuators, and simulation models are described in the literature surrounding the studied topic [40, 60, 67]. The following section highlights the main features of the reviewed models and approaches to find the most suitable solution to model the built prototype mathematically. 
There are two main methods for modelling hydraulic systems: employing Simulink toolbox (MATLAB) and corresponding transfer functions [64, 66, 67], or using Simscape (MATLAB) for higher level simulation [56, 63, 68, 69]. Overall, the use of these modelling tools helps users with different competency levels to modify a computer model by changing its parameters via the software's graphical user interface (GUI).

There are a few assumptions regarding hydraulic models in Simscape and other simulation tools [70]:

1. Fluid compressibility is negligible.

2. Leakage of a pump or a motor is proportional to the differential pressure. A mathematical model of a gas-charged accumulator is based on the following assumptions [56]:

1. The gas in the accumulator is ideal.

2. No inertia, friction, or load on the separator or the bladder.

3. Fluid is incompressible.

Nevertheless, it is possible to simulate system losses [71] by incorporating relevant equations and correlations. Meanwhile, the behaviour of a gas charged accumulator heavily depends on the employed gas model, since real gas cannot be modelled as an ideal when its pressure is higher than $100 \mathrm{bar}$ (due to the gas compressibility) [72].

Another difference between different modelling approaches is the use of linearised or non-linear models [56, 61, 63, 66, 68, 69]. The former sometimes do not represent the behaviour of a system correctly when it is subject to large amplitude inputs. For instance, when there are few sources of non-linearity in hydraulic systems, including the relationship between pressure and flow, flow deadband and saturation, change of fluid volume during different stages of a stroke, temperature changes in the bulk modulus of hydraulic fluid and so on [64, 67, 73].

\subsubsection{Generic simulation models}

There are no mathematical models of the drivetrains similar to the system employed in the prototype. Meaning that it is required to review its hydraulic components and then its electric components separately. Firstly, the models presented in the literature that are based on Simscape libraries are reviewed in this section. 
Boya Zhang in her thesis [56] describes a hydraulic energy storage system for hybrid locomotives. Two main points regarding its model could be used for our proposed model. The first relates to the modelling of a bladder accumulator discharge process. The second one is a control mechanism for the swash-plate system in a variable displacement hydraulic motor. This information can be used for an improved mathematical model of the prototype.

The acceleration and speed of the hydraulic motor and the generator in the prototype are controlled through the use of the employed proportional throttle valves. The proposed technique in our project can be modelled by using a proportional throttle valve model, powered by a constant pressure source. However, there are few methods of designing a variable-speed hydraulic drivetrain. For instance, another approach is based on the use of a variable-displacement hydraulic pump which pumps hydraulic fluid through a hydraulic motor. These systems can be modelled as an open-loop [66] or a closed-loop system [63].

A nonlinear approach to the modelling of similar systems is presented in the paper by Ayman A. Aly [64]. Two significant nonlinearities are considered in the system: pressure/flow characteristics and Coulomb-friction. Closedloop control of the system is obtained by comparing the angular position of a motor shaft with the input signal by a control circuit. A tachogenerator in this prototype is used to measure an angular velocity, which can be utilised as a feedback signal to the input of a servo valve drive amplifier. Concerning the development of a closed-loop approach, the model presented in the paper employs similar technique to ours and can be used as a reference during the modelling stage.

\subsubsection{Mathematical Modelling of Hydraulic Drivetrains}

The prototype's hydraulic drivetrain is composed of two main components a proportional throttle valve (TDA016) and a variable displacement hydraulic motor (M14 Goldcup). To fully understand the operation and behaviour of the main components, it is required to research the literature surrounding these components.

Saber Madanipour [65] presented a model of a hydraulic servo motor while mentioning that there are some different types of nonlinearities presented in these devices [61]. On top of that, this paper fully supports the idea that there is no need to implement a PID controller in a speed control loop, while overall stability and behaviour of the system could be enhanced when it is implemented in a fluid control loop [74]. 
The main issue with all hydraulic drives is their nonlinearity and low damping, so it is difficult to control these systems fully [74-76]. In most of the cases, a control system compares the error signals with the target velocity, position, force, pressure, and other parameters [62]. Abdul's [62] main approach was to develop a control mechanism that can adapt to the nonlinear behaviour of a system by continuously tracking its velocity and position.

Apart from the generic models of hydraulic and hydro-mechanical drivetrains, many researchers focus on the realistic modelling of proportional and servo valves [67, 75,78], since they are subject to different types of non-linearities.

\section{Mathematical modelling of proportional throttle valves}

A research team from the University of Poznan [77] highlighted that there are two main schemes of controlling a hydraulic actuator, i.e. the use of a servo valve or a proportional valve [62]. The first one is more expensive and, usually, is employed in high-accuracy applications. Meanwhile, proportional valves respond acceptably to the imposed requirements in most of the applications throughout different industries.

Flow rate through a valve with sharp-edged nozzles is turbulent, which can be described using the following equations [62, 65, 77]:

$$
Q=K_{Q} \sqrt{\Delta p} x
$$

where $K_{Q}$ is a flow coefficient, $\Delta \mathrm{p}$ is a pressure drop on a valve (bar), and $x$ is a spool displacement $(\mathrm{mm})$. The flow coefficient $K_{Q}$ depends on the following parameters:

$$
K_{Q}=\mu_{d} \sqrt{\frac{2}{\rho}} w
$$

Where $\mu_{d}$ is the discharge coefficient, $\rho$ is oil density $\left(\mathrm{kg} / \mathrm{m}^{3}\right)$, and $w$ is the width of the gap (mm). Furthermore, for $x>0$ :

$$
\begin{gathered}
Q_{a}(t)=K_{Q} \sqrt{p_{0}-p_{a}(t)} x(t) \\
Q_{b}(t)=K_{Q} \sqrt{p_{b}(t)} x(t)
\end{gathered}
$$

for $x<0$ :

$$
\begin{gathered}
\left.Q_{a}(t)=K_{Q} \sqrt{p_{a}(t)} x(t)\right) \\
\left.Q_{b}(t)=K_{Q} \sqrt{p_{0}-p_{b}(t)} x(t)\right)
\end{gathered}
$$


Where $\mathrm{x}$ is a spool displacement $(\mathrm{mm}), Q_{a}$ flow through gap $\mathrm{A}\left(\mathrm{dm}^{3} / \mathrm{min}\right)$, $Q_{b}$ flow through gap B $\left(\mathrm{dm}^{3} / \mathrm{min}\right), K_{Q}$ is the flow coefficient, $p_{0}$ is the supply pressure $(\mathrm{Pa})$ and $p_{a}, p_{b}$ the pressure in chambers $\mathrm{A}$ and $\mathrm{B}(\mathrm{Pa})$.

Overall, the system presented in the paper represents an attractive alternative to servo-valves. It helps to achieve better dynamic characteristics than the standard electro-hydraulic proportional valves with solenoids.

In general, a model of a proportional or servo valve is divided into two parts: a control stage and a power stage. The former depicts the dynamics of the valve's spool, and the latter describes a flow rate through a valve at a certain differential pressure across the valve at a specific opening [78].

The modelling process of proportional and servo valves is challenging, because of the lack of standardisation among manufacturers. So the most accurate approach to the determination of valve parameters is experimental validation [64, 68, 75].

One of the most noticeable effects in the valve's operation is hysteresis that occurs in the coil being energised by a DC voltage. It changes the openingclosing flow rate through a valve, hence the speed of a generator and a motor. It happens within the whole operation range of the valve, hence affects the whole range of the dynamics of a drivetrain. It should be noted that there is no literature covering this effect regarding control and acceleration of electrical machines (like synchronous generators) [79].

To derive the flow rate profiles within the range of 0-350 bar, it is required to calculate the flow coefficients of the TDA016 (also known as a valve sizing coefficient). In general flow, coefficients might differ for the same size of valve, only due to the different geometrical shape of the valve's poppet [80].

\section{Modelling of hydraulic motors}

In most of the cases, hydraulic motors are modelled as a first-order transfer function, in the same manner as DC machines are simulated. There are two main parameters of a hydraulic motor (electro-hydraulic drivetrain): displacement and inertia. The displacement of a hydraulic motor can be determined as [81]:

$$
V_{D}=\frac{Q}{N}
$$

where $\mathrm{N}$ is the motor's velocity in RPM, $\mathrm{Q}$ is the flow rate through the motor in lpm, and $V_{D}$ is the motor's displacement in $1 / \mathrm{rev}$.

From here the displacement determines the maximum speed of the motor at a particular flow rate through it. This parameter can be changed in variable displacement hydraulic motors (due to the presence of a swash plate). The 
displacement should be at a certain level that allows the motor to accelerate to the required speed (synchronisation speed or $50 / 60 \mathrm{~Hz}$ ).

The inertia of the hydraulic drivetrain determines how fast the system can accelerate to a certain velocity, i.e., the system's dynamics. In the case of the proposed system, it determines the synchronisation time with the power grid due to different derived acceleration trajectories, since

$$
T=J \cdot \frac{d \omega}{d t}
$$

where $\mathrm{J}\left(\mathrm{kg} / \mathrm{m}^{2}\right)$ is the combined inertia of the motor and the generator, $d \omega / d t$ $\left(\mathrm{rad} / \mathrm{s}^{2}\right)$ is the motor's acceleration, and T is the drivetrain's torque in $N \cdot m$.

The torque can be measured by the use of torque sensors that are installed in the motor. The test system has only an incremental encoder to measure the drive train's position, velocity and acceleration. Based on these variables, the torque can be found as [82]:

$$
T=\frac{\eta_{o \cdot \Delta P \cdot Q}}{N_{R P M}}=\frac{\Delta P \cdot V_{D_{c}}}{62.83}
$$

where $\eta_{o}$ is the overall efficiency, $\Delta P$ is the differential pressure across the motor (bar), and $V_{D_{c}}$ is the volumetric displacement of the motor in $c c / r e v$.

\subsubsection{Modelling of electrical components}

Since the key electrical component of the prototype is a three-phase synchronous generator, it is imperative to review the literature regarding its modelling. The mathematical representation of a synchronous machine has been a subject for constant research for the last century since the first description of a D-Q model was presented by Park [83], [84] in 1929 and then modified by Demello and Concordia in 1969 [85]. The latter is also a reference point for all studies focused on the operation of a synchronous generator connected to an infinite bus since it focuses on the stability's phenomenon of a torque-angle loop, hence the behaviour of the rotor angle and speed, due to mechanical torque disturbances.

Later, the most comprehensive model was described in 1994 by Saidy and Hughes [86], which includes damper windings on both $\mathrm{d}$ and $\mathrm{q}$ axes. Moreover, their next paper [87] focused on a model which includes field and one damper winding along the d-axis and two damper windings along the q-axis.

Since 1991 the most widely used method for modelling synchronous generators has been described in the current IEEE Standard [88] and one of the most employed models of type 3.3. Nowadays researchers concentrate more on different approaches to identifying parameters of a model [79, 89-91]. For instance, in 2002 a research group from TU Delft (Netherlands) [92] described 
the time-domain identification of synchronous generator's transfer functions, which helps to modify a model structure so that it can be used for simulation. From this perspective, all generators have similar representations and differ only concerning some parameters, for example, the difference between rotor and silent pole machines. This is the main approach to the modelling of these machines in Simulink/Simscape.

Some studies focus on the comparison of different synchronous generator models connected to an infinite bus [93-97]. An interesting point that applies to modern modelling techniques is highlighted in the studies. AVR systems and governor / turbine control cannot be modelled accurately due to their complexity. In this case, it is worthwhile to use the ideal sources of an excitation voltage or current, and an ideal source of mechanical power or a speed source as a governor. At the conclusion of the paper, it is stated that AVR and governor effects are significant when the rotor oscillations are large [98].

\subsection{Controller Encoder Applications in Hydraulic Drivetrains}

There are various applications for the use of encoders in electro-hydraulic and hydraulic drivetrains. Many sources reference the use of encoders only as a preparation step for the implementation of different Kalman filtering techniques. The controllers that are usually utilised for such studies are used only for filtered velocity and acceleration measurements [99-101]. Whereas in the case of the studied system, the controller serves as a data acquisition unit for various signals and calculates the required acceleration trajectory at the same time. This is why the acceleration filtering was performed with the use of classic techniques such as Mean and Savitzky-Golay [102]. Kalman filtering would introduce a significant burden on the controller.

To successfully implement Kalman filtering, researchers focus on the applications with the use of low-resolution encoders [100, 103]. In this case, the use of Kalman filters is imperative.

Mean filtering is one of the oldest technologies in signal processing and calculates the mean value of data points over the specified window sample length. Savitzky-Golay filtering also known as a least square method is the next stage in "moving average" filtering [102]. In some cases, this approach can work better and faster (due to a smaller time delay) and mean filtering. This statement was proven during the experimental testing of the system to find the most suitable filtering techniques. 


\subsection{Chapter Summary}

The chapter presents an overview of the majority of existing energy storage systems, highlighting the advantages of the use of the proposed ss-CAES system. The proposed synchronisation approach for the rapid acceleration of synchronous generators does not have any alternatives in the literature surrounding this topic. Since this acceleration and synchronisation approach is verified with the use of an electro-hydraulic drivetrain, an overview of the challenging hydraulic domain and its mathematical modelling is provided. 


\section{Chapter 3}

\section{ss-CAES System Synchronisation}

This chapter focuses on the mathematical description of the proposed synchronisation approach and its verification via simulations with the employed manufacturer's data for the main components of the system. To summarise, the main steps for the system's verification are presented in Fig. 3.1 .

A mathematical model was initially designed by the UoC in MATLAB to simulate the proposed acceleration approach. The main limitation of this approach is the use of the manufacturer's data and ideal models of the electrohydraulic components. It verifies the mathematical basis of the control approach, however, does not help to find the correct control parameters for the system.

Hence, to verify the synchronisation approach prior to hardware implementation, it is required to have a verified and accurate mathematical model. Meaning that the preliminary experimental testing and modelling conducted by the UoC did not give any feasible and accurate information about the operation of the system. To experimentally characterise the proportional valve and the motor, a data acquisition system was implemented into the main hydraulic circuit. It is based on a National Instruments controller with a net of different sensors, components and input and output controller modules.

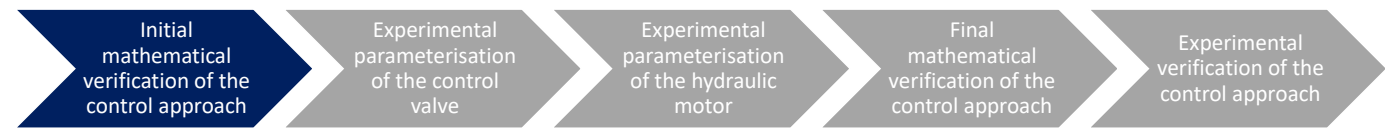

Figure 3.1: Verification steps of the proposed synchronisation approach with current step presented in the chapter. 


\subsection{Synchronisation Approach}

Design of the acceleration and synchronisation approach is the most critical and difficult part of the commissioning of the proposed device. To be employed as a FIR system, the system should be connected to a power grid within $1-6 \mathrm{~s}$. In this scenario, the " $6 \mathrm{~s}$ requirement" is the time threshold for generating FIRs, whereas ILs require the trigger time of $1 \mathrm{~s}$.

To synchronise a generator with a power grid, the following requirements must be met:

1. The sequence of the three phases of the generator must be the same as of the power grid.

2. The frequency of the sinusoidal voltage produced by the generator must be equal to the frequency of the sinusoidal voltage produced by the grid.

3. The magnitude of the sinusoidal voltage produced by the generator must be equal to the magnitude of the sinusoidal voltage of the grid.

4. The phase angle between the voltage generated by the generator and the voltage generated by the grid must be zero.

Historically, the conventional synchronisation procedure is employed to synchronise a generator to a power grid, and it is shown in Fig. 3.2 [104].

The phase sequence is managed during the installation of any electrical system, and it is the responsibility of a project engineering team. Hence, this part of the synchronisation procedure will not be mentioned further in the thesis. The use of the classic method means that it is possible to reach the best moment for synchronisation; however, it does not minimise the time to reach this state; thus the modified synchronisation procedure was engineered. In theory, the implementation of the proposed approach can minimise the synchronisation time by $50-70 \%$ (from 6-10 s following the conventional approach to 1-4 s according to the 2-stage ballistic approach).

By employing the proposed strategy, it is possible to fulfil the requirements 2 and 4 of the conventional synchronisation procedure. The equality of the voltage magnitudes of a grid and a generator (requirement 3 ) is achieved through the excitation system which is utilised in the prototype. This is a simple process; therefore it will not be mentioned further in this thesis. The assembled hardware prototype for these studies is presented in Fig. 3.3 .

To synchronise a synchronous generator (up to $100 \mathrm{~kW}$ ) with the power grid, it is required to fulfil the synchronisation requirements presented in Ta- 


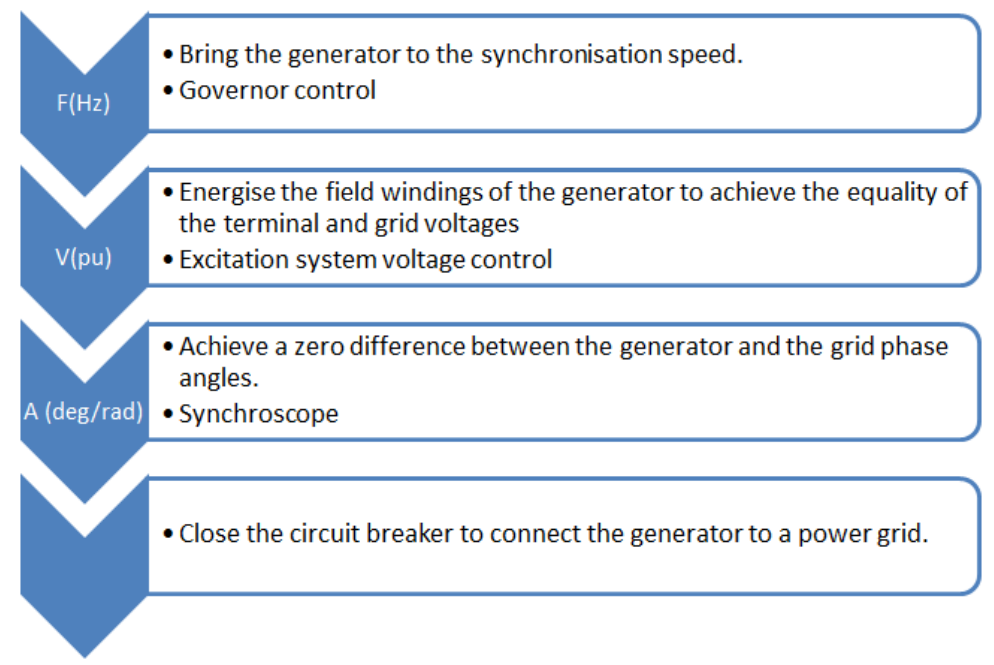

(a) Conventional synchronisation procedure.

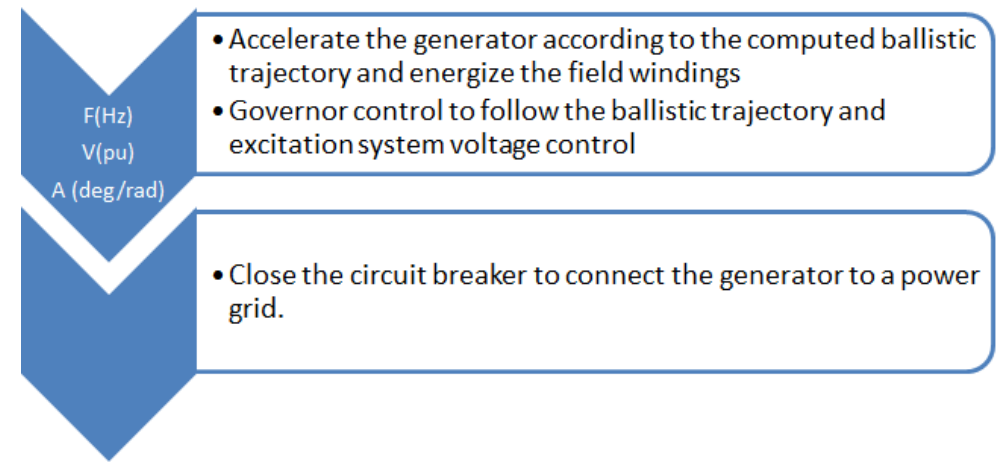

(b) Proposed improved ballistic synchronisation approach.

Figure 3.2: Comparison between the conventional synchronisation approach (a) and improved (2-stage) ballistic synchronisation approach.

ble 3.1. However, the manufacturer of the prototype's generator (ABB) also provides another set of synchronisation limits, combined in Table 3.2 .

The rapid start-up is challenging, due to the required acceleration of the entire rotor inertial mass. Feedback control should be implemented, which will minimise the start-up time from the "at rest" initial condition and avoid current-spiking from the generator. The ability to start up from "at rest" would enable lower operating costs for a grid-deployed hydraulic-EES, as this will not incur inherent $I^{2} R$ and other losses that occur during condenser operation.

Control of synchronous operation includes the implementation of a governor to provide over-frequency control. Overall, the acceleration demand trajectory (ballistic and 2-stage ballistic) is based on calculating a fixed shaft torque such that the error between the grid and the generator phase angles is zero at the time when the generator frequency matches the grid frequency. This synchronisation philosophy was firstly introduced in the MSc project which was part of the earlier preliminary work done on this prototype in 2014-2015 [20]. 


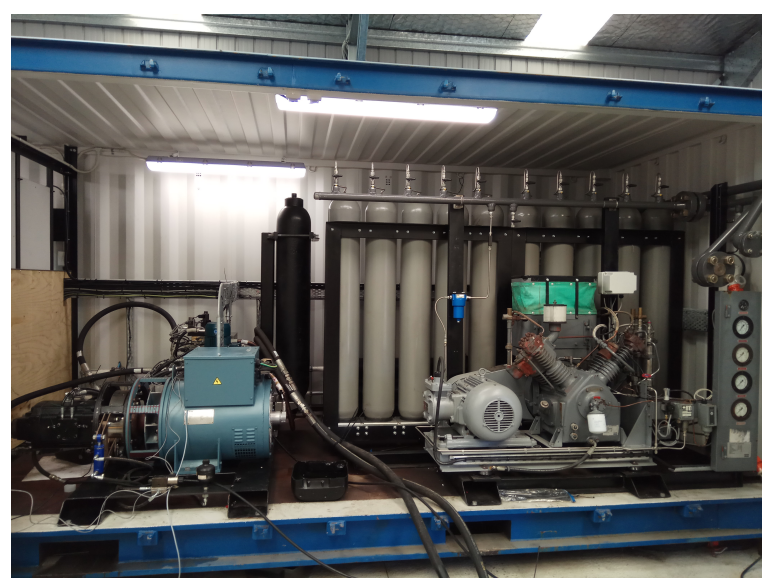

Figure 3.3: Assembled prototype is undertaking its experimental testing in New Plymouth, New Zealand (2017-2018).

Table 3.1: IEEE 1547 Synchronisation boundaries for generators $<100 \mathrm{~kW}$

\begin{tabular}{lll}
\hline Parameter & Recommended & Absolute \\
\hline \hline Voltage & $15 \%$ & $40 \mathrm{~V}$ \\
Frequency & $0.3 \mathrm{~Hz}$ & $0.3 \mathrm{~Hz}$ \\
Phase angle & $20^{\circ}$ & $20^{\circ}$ \\
\hline
\end{tabular}

The visual depiction of this method is presented in Fig. 3.4

The basic principles behind the ballistic synchronisation approach are described in [21, 105]. It can be summarised as the process of synchronisation by controlling the acceleration trajectory of the generator to target a future moment in time at which both the generator phase and frequency simultaneously match the grid. Feedback control of the trajectory of $\theta_{\text {gen }}$ is enabled through real-time measurement of the angular position of the generator shaft.

To implement this method in hardware, a linear interpretation of the generator and grid phases is used [106].

$$
\left.V_{\text {gen }}=V_{\text {gen }} \cdot \sin \theta_{\text {gen }}(t)\right)
$$

Table 3.2: Synchronisation boundaries for the employed ABB AMG-0250 Synchronous generator

\begin{tabular}{lllll}
\hline Parameter & Recommended & Absolute & Maximum & Absolute \\
\hline \hline Voltage & $2 \%$ & $8 \mathrm{~V}$ & $4.5 \%$ & $18 \mathrm{~V}$ \\
Frequency & $0.7 \%$ & $0.35 \mathrm{~Hz}$ & $4 \%$ & $2 \mathrm{~Hz}$ \\
Phase angle & $<15 \%$ & $<15 \%$ & Unspecifed & Unspecified \\
\hline
\end{tabular}




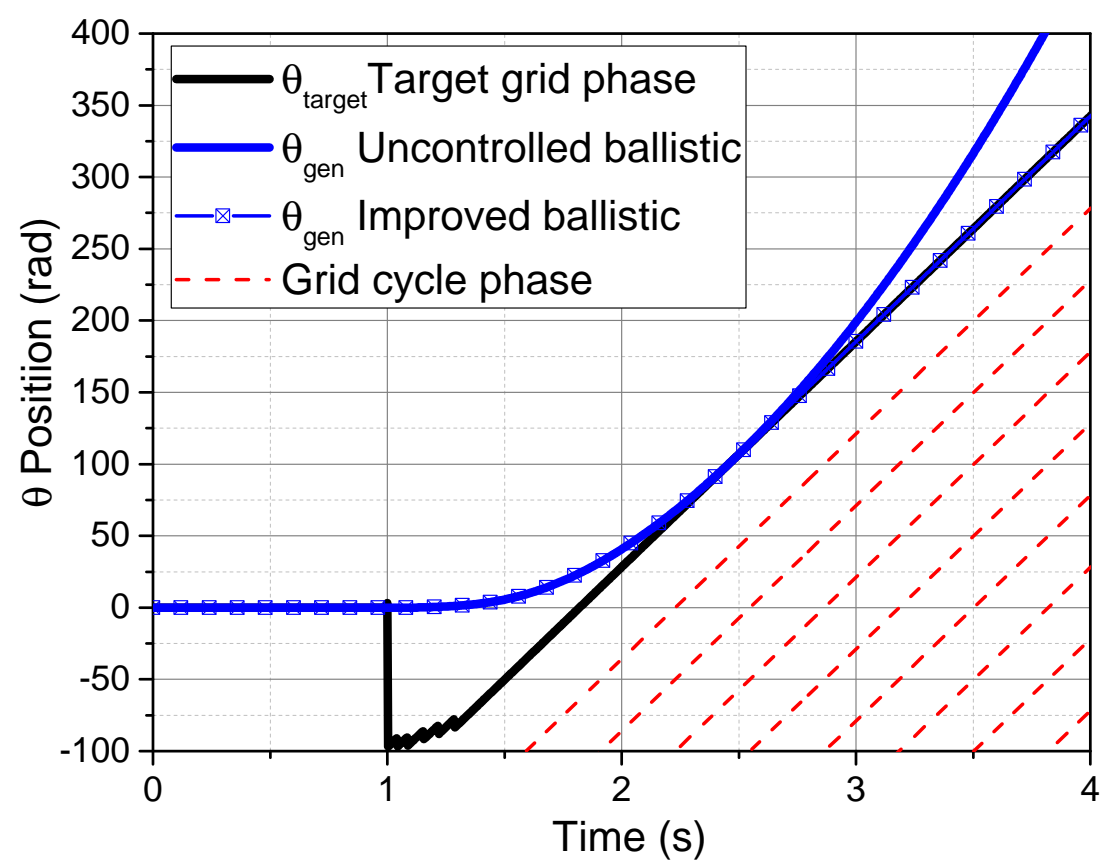

Figure 3.4: Proposed synchronisation philosophy for synchronous generators simulated in Simulink - comparison of the ballistic and improved ballistic approaches.

$$
\omega_{g e n}(t)=\frac{\mathrm{d} \theta_{g e n}(t)}{\mathrm{d} t}
$$

where $V_{\text {gen }}$ is the voltage waveform of the generator $(\mathrm{V}), V_{g e n_{m}}$ is the amplitude of the generator voltage cycle $(\mathrm{V}), \theta_{\text {gen }}$ is the generator phase (position) (rad) and $\omega_{g e n}$ is the angular frequency of the generator ( $\left.\mathrm{rad} / \mathrm{s}\right)$.

Analogous equations define the grid phase, $\theta_{\text {grid }}$, and angular frequency, $\omega_{\text {grid }}$. Note that here we consider the electrical phase of the generator such that a $2 \pi$ cycle represents a half revolution of the generator's 4-pole rotor.

As the generator is accelerated from rest, its angular speed (frequency) increases rapidly, such that:

$$
\begin{gathered}
\theta_{\text {gen }}(t)=\theta_{\text {gen }}(0)+\int_{t_{0}}^{t} \omega_{\text {gen }}(t) \mathrm{d} t \\
\omega_{\text {gen }}(t)=\int_{t_{0}}^{t} \alpha_{g e n}(t) \mathrm{d} t
\end{gathered}
$$

where $\alpha_{g e n}$ is the acceleration of the generator phase angle $\left(\mathrm{rad} / \mathrm{s}^{2}\right)$ and $t_{0}$ is the current sample time (s).

The phase acceleration of the generator, $\alpha_{g e n}$, is proportional to the torque exerted on the generator shaft by the prime mover (i.e. hydraulic motor). 
At the moment of synchronisation, $t_{\text {sync }}$ the position and speed of the generator rotor must exactly match a grid cycle, such that the following conditions must be met:

$$
\begin{gathered}
\Delta \omega=\left|\omega_{\text {gen }}-\omega_{\text {grid }}\right|<\delta_{\omega, \text { sync }}=0.94 \mathrm{rad} / \mathrm{s} \rightarrow 0.3 \mathrm{~Hz} \\
\Delta \theta=\left|\theta_{\text {gen }}-\left(\theta_{\text {grid }}-2 \pi n\right)\right|<\delta_{\theta, \text { sync }}=20^{\circ}
\end{gathered}
$$

where $n$ is an integer, $\omega_{\text {grid }}$ is the angular frequency of the grid ( $\left.\mathrm{rad} / \mathrm{s}\right), \theta_{\text {grid }}$ is the grid phase (rad).

A visual representation of this approach is depicted in Fig. 3.4. The diagonal lines each shifted by $2 \pi$ represent equivalent phase angles occurring in sequential $50 \mathrm{~Hz}$ grid cycles. In this plot, the system is triggered at $1 \mathrm{~s}$. To achieve ballistic synchronisation, the generator must accelerate so that its phase trajectory will tangentially intersect one of the diagonal grid cycle lines. Tangential intersection indicates the synchronisation point where both the frequency and phase of the generator and the grid are equal. This can be achieved by following the acceleration trajectory given in (7.2) [20]:

$$
\alpha_{\text {optml }}=\frac{1}{2} \frac{\omega_{\text {gen }}^{2}}{\theta_{\text {gen }}-\left(\theta_{\text {grid }}-2 \pi n\right)}
$$

where the $2 \pi n$ term determines the target grid cycle for synchronisation and is chosen so that the computed acceleration does not exceed a safe maximum upper limit. The control algorithm is executed at a timestep of $1 \mathrm{~ms}$ in the main controller, and the choice of target cycle is continuously recomputed. The target grid cycle is identified by its absolute phase, $\theta_{\text {target }}$. This is shown as the black line in Fig. 3.4. It is common for the controller to repeatedly recompute the target grid cycle during the early stages of acceleration (when the relative phase velocity is largest). This can be observed as the series of $2 \pi$ jumps in $\theta_{\text {target }}$ which occur between 1.0 and $1.3 \mathrm{~s}$ in this plot.

Fig. 3.4 also shows two different computed acceleration trajectories. The blue line shows a continuous acceleration trajectory in which the generator maintains constant acceleration so that it accelerates up to and then past the grid cycle. In this case, the circuit breaker must be closed quickly, before the generator accelerates past the grid and exits the synchronisation window. However, the synchronisation window for this approach is very short when rapid acceleration trajectories are employed, being typically $<20 \mathrm{~ms}$ when targeting synchronisation in $<6 \mathrm{~s}$ [105]. This window is too short to close many mechanical type circuit breakers. To address this, we have developed and implemented a new improved ballistic control approach which follows the accel- 

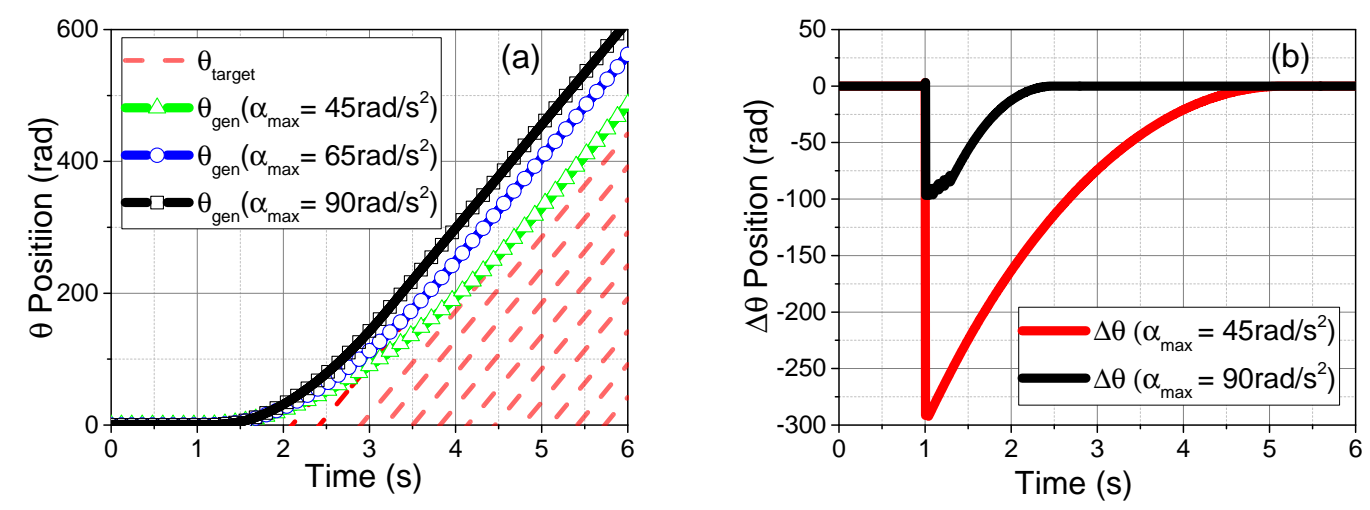

Figure 3.5: Plots showing simulated example profiles for the 2-stage ballistic synchronisation approach. (a) Generator phase acceleration obtained under different limiting maximum acceleration values. (b) Computed phase difference between the generator and target grid phase for two different limiting acceleration values

erating trajectory of (7.2) until the generator enters the synchronisation window, at which point the feedback control then changes to track the grid phase and frequency seamlessly. The line with symbols shows this improved control trajectory in Fig. 3.4. The transition to a 'grid - following' control mode now indefinitely extends the available synchronisation window, providing ample time for circuit breakers to be closed and hence connect the generator to the grid.

When the phase of the generator reaches the upper limit of a synchronisation window, the controller switches from the ballistic acceleration loop to the tracking loop. This loop is a PID loop with the grid's frequency (speed) used as a set point for the PID controller. The upper limit trigger for the tracking loop was easier to implement in the hardware controller and achieve simple switching between the ballistic and tracking loops.

Fig. 3.5 shows the effect of limiting the maximum acceleration rate on the computed acceleration trajectory. In each case, the control system can find the optimum target phase grid cycle. The effect of decreasing the maximum permissible acceleration merely is that the controller targets and achieves synchronisation in successively later grid cycles.

In order to finalise the depiction of the synchronisation picture, the phase difference $\left(\Delta \theta=\theta_{\text {gen }}-\theta_{\text {target }}\right.$, where $\left.\theta_{\text {target }}=\theta_{\text {grid }}-2 \pi \cdot n\right)$ is shown in Fig. 3.5(b) with slow and fast acceleration of the generator. 


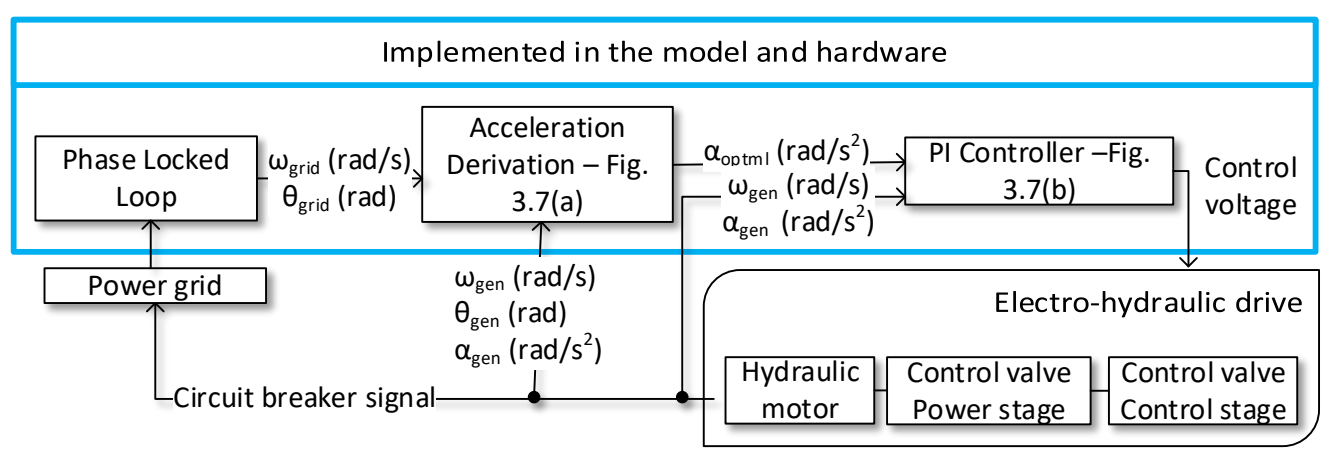

Figure 3.6: Block diagram of the mathematical model of the proposed ss-CAES system in Simulink.

\subsubsection{Mathematical model of the proposed system}

A mathematical model that simulates the proposed acceleration approach was designed in the MATLAB/Simulink environment by the UoC (based on the manufacturer's data) and then modified with the experimental data by the $\mathrm{PhD}$ author. Its block diagram is presented in Fig. 3.6

- The power grid is modelled as a three-phase voltage source with variable frequency and phase. This allows modelling of contingent events that are characterised by phase and frequency fluctuations (ramp and step droops). For the later experimental testing of the prototype, this grid was replaced with a simulated frequency signal in the Labview environment.

- The phase locked loop (PLL) system is used to determine the phase and the frequency of the power grid. The full description of the system and also its simulated behaviour can be found in [21]. In order to reduce the computation burden on the controller during the later experimental testing, this system was replaced with a simplified version based on an integrator for ramping the grid phase. Since the frequency and phase signals are simulated, there is no need for filtering and other mathematical derivation that generally take place in the actual PLL structure.

- The Acceleration Derivation system calculates the required acceleration of the generator. To derive it, the system inputs the grid frequency and phase, the generator's phase, velocity, and the acceleration feedback measurements. Figure 3.7(a) shows a flowchart summarising the logic used to determine an acceleration trajectory and the transition from ballistic acceleration to the grid-following mode (tracking loop) (2-stage ballistic approach). In Labview, this algorithm was implemented with the use of 
the Mathscript toolbox which enabled the use of this script (with some corrections) directly from the designed mathematical model.

- The optimal acceleration output from the Acceleration Derivation system inputs to the controller block shown in Fig. 3.7 (b). The Acceleration Derivation system accelerates the generator according to the calculated ballistic approach. When the generator reaches the upper limit of a synchronisation window $\left( \pm 0.3 \mathrm{~Hz}, \pm 15^{\circ}\right)$, the controller switches to the tracking loop to track the grid's frequency. At this moment the Acceleration Derivation system stops transmitting any voltage to the control valve.

The tracking loop (hysteresis compensation loop) reduces the speed of the generator, so it would match the reference speed of the grid. Since the control loop continuously tracks the frequency as its setpoint, it is possible to significantly increase the synchronisation window. It allows enough time to close the circuit breaker and connect the generator to the grid.

It is observed that some discontinuity occurs during a transition between the ballistic acceleration loop and the tracking loop. The discontinuity is due to the time loop rate of the hardware controller and is typically around $1 \mathrm{~ms}$. The faster the controller loop, the shorter the discontinuity. The influence of the controller parameters of the synchronisation process is described in Section 6.1.1. The controller system was implemented in hardware along with the main acceleration derivation system.

Parameter $G_{1}$ shown in Fig. 3.7 and it describes the gain controller:

$$
\text { Output }=\left(\alpha_{\text {optml }}-\alpha_{\text {gen }}\right) \cdot 0.0023 \cdot \omega_{\text {gen }}+0.2
$$

- The electro-hydraulic drivetrain model simulates the hardware part of the project, which mainly comprises a proportional throttle valve (TDA016) and a variable displacement motor. The primary challenge for this part of the project is the empirical parameterisation of the components that are required in order to update the controller parameters and reach synchronisation.

The most common practice for modelling a proportional valve is the use of two transfer functions. The first (control stage) describes the dynamics of a valve, i.e., how fast the valve can be opened to a specific orifice area. This can be described as a second-order transfer function [78]:

$$
H(s)=\frac{1}{1 / \omega_{n}^{2} \cdot s^{2}+2 \cdot \zeta / \omega_{n} \cdot s+1}
$$



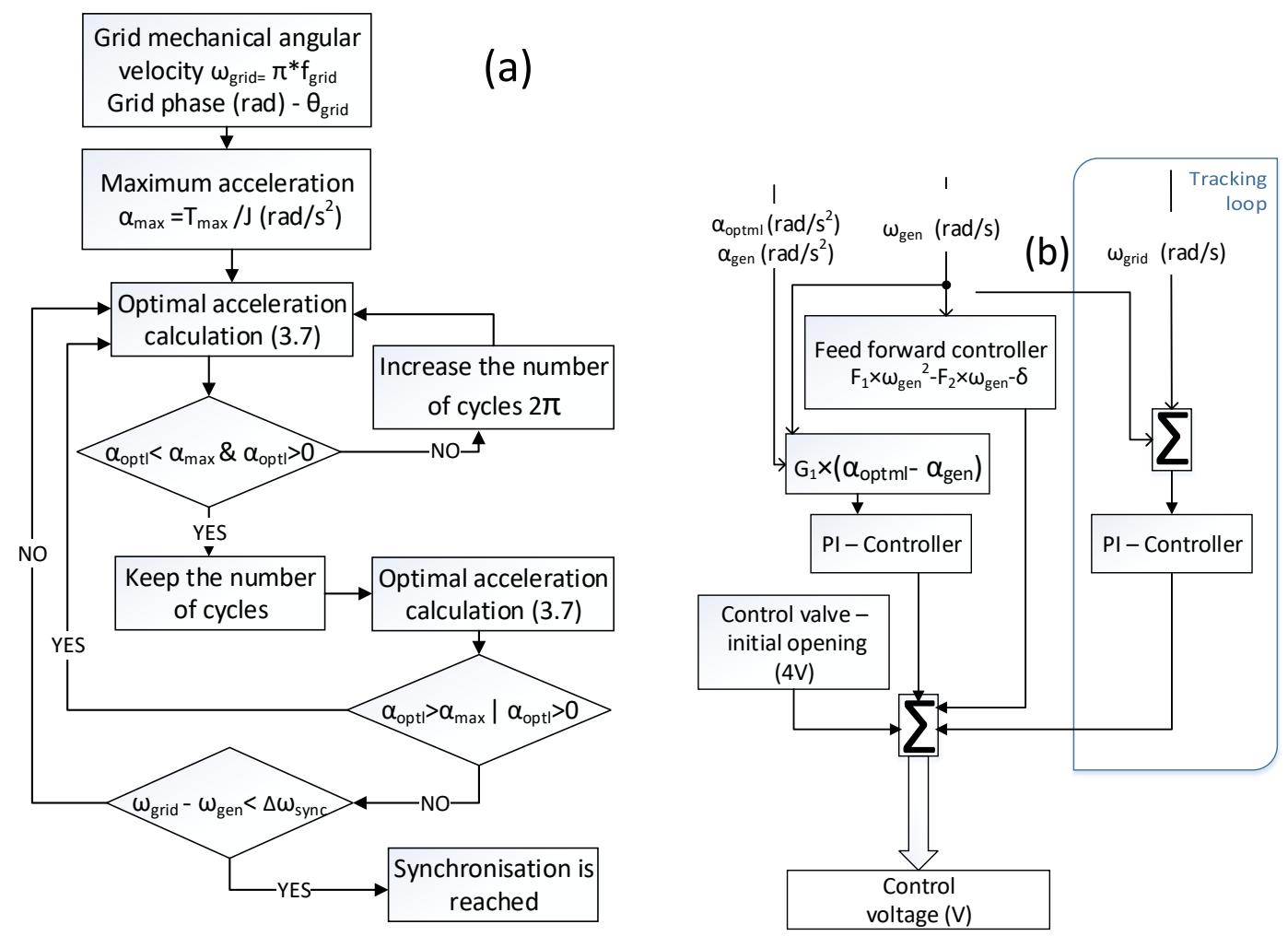

Figure 3.7: Control system. a) Flow chart of the acceleration derivation algorithm - Acceleration Derivation system in Fig. 3.6. (b) Controller's block diagram for derivation of the control voltage during the acceleration and synchronisation - PI - Controller in Fig. 3.6.

where $\omega_{n}$ is the natural frequency of the valve and $\zeta$ is the damping ratio. The second part of the model (power stage) can be implemented as a $2 \mathrm{D}$ lookup table with the flow rate, differential pressure across the valve and its openings [78]. The use of this two-stage model helps to achieve the most accurate modelling of the valve's behaviour [78].

In most of the cases the process of a valve's parameterisation is based on its manufacturer's data, however, this can be inaccurate due to the high complexity and non-linearity of hydraulic components [107]. In this case, the TDA016 should be characterised experimentally with the use of the designed data acquisition, which is described in Chapter 5 . The hydraulic motor's characterisation is presented in Chapter 6.

The designed mathematical model can be verified only with the experimental testing of the built prototype. 


\subsection{Preliminary Simulation of Synchronisation}

\subsubsection{Original Ballsitic Synchronisation Approach}

The presented results in this section were peer-reviewed and accepted for publication and presentation at ISGT PES IEEE Asia 2016 and 2017 conferences, which were hosted in Melbourne, Australia and Auckland, New Zealand respectively [105, 108]. They represent the initial modelling results that were achieved during the first 6-9 months of this PhD project.

A mathematical model's block diagram of the proposed system is presented in Fig. 3.6. All the components are fully described in the afore mentioned MSc thesis [20]. However, it is worth mentioning that the acceleration derivation block is used to compute the required acceleration trajectory by using the output values from the power grid and the generator. In this system, the feedforward block is used to maintain a constant valve pressure loss employing a flow rate as a variable, hence maintaining a constant acceleration.

Fig. 3.8 shows the simulated behaviour of the system during its rapid startup and synchronisation. The generator is at rest until $t=1 \mathrm{~s}$, at which it is triggered.

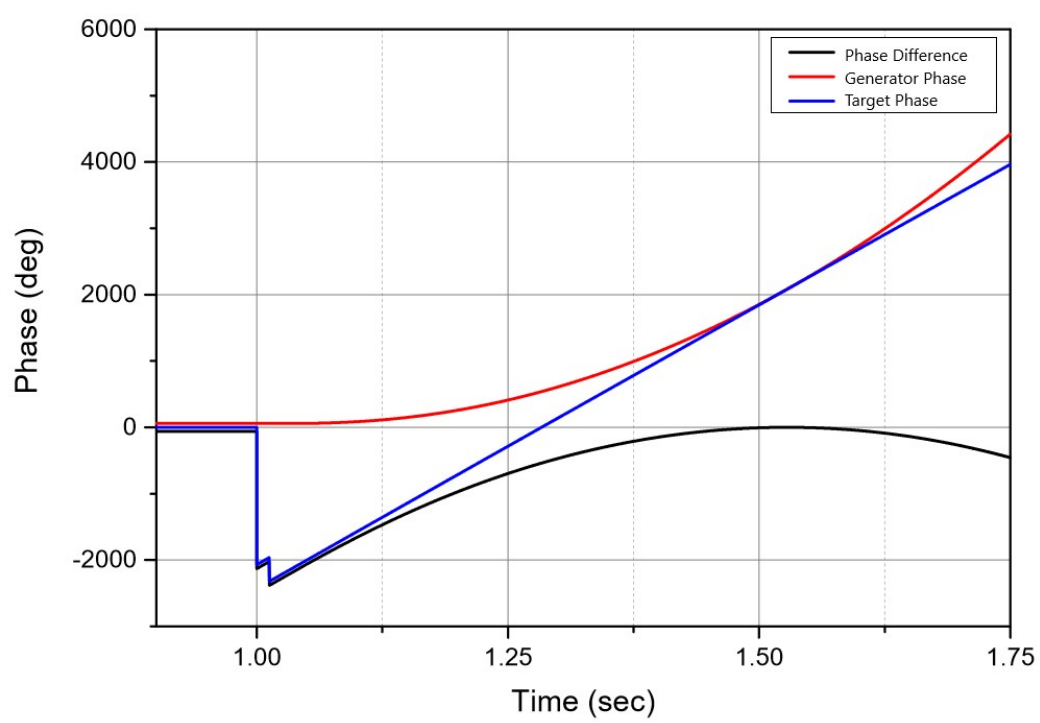

Figure 3.8: Simulink modelling results of the generator and the grid phases following an event to trigger rapid start-up and grid synchronisation

The control system computes the phase error, and it is the difference between the generator phase and the grid cycle that was chosen for synchronisation. The synchronisation moment occurs when the error is equal to zero. When the system is triggered, the control system computes the optimal phase at which synchronisation can occur, taking into consideration the computed 


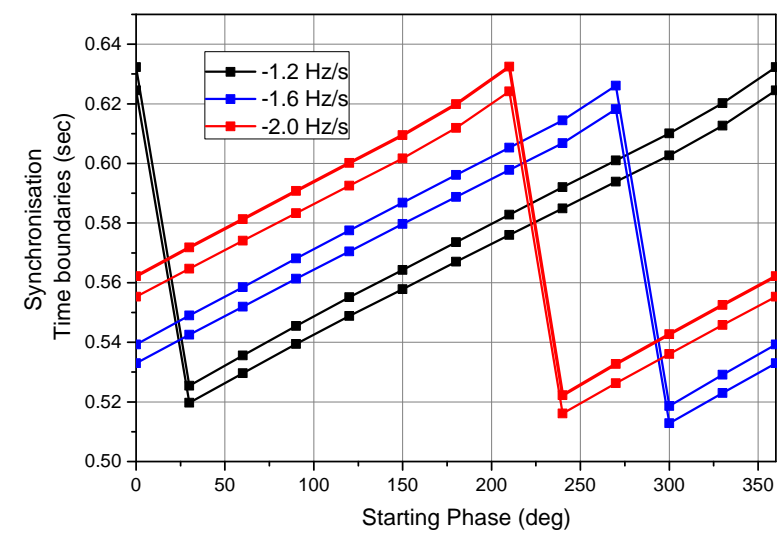

Figure 3.9: Lower and upper time boundaries for synchronisation within the different frequency decrease rates

rotor's acceleration trajectory. It then sets the phase error to a negative value with magnitude $>>2 \pi$, taking into consideration that the grid must complete several extra cycles during the rotor's acceleration process. It can be seen that Fig. 3.8 shows a discontinuity in the phase error at $1-1.15 \mathrm{~s}$, where the computed error drops by $\sim 2 \pi$. This is caused due to the continuous re-computing of the target phase for synchronisation, and it is based on the actual generator's acceleration. When the control system predicts that the measured acceleration cannot fulfil all the requirements regarding phase and frequency matching, the target phase is recomputed such that it "slips back" by another cycle. The same behaviour continues until the measured acceleration trajectory is predicted to meet the criteria required to achieve synchronisation at the target phase.

Throughout the simulation of this model with different frequency droop rates and the initial phases of the generator's shaft, a number of graphs were plotted to verify its robustness.

Fig. 3.9 portrays the time for synchronisation of the generator with a power grid. The lower boundary represents the moment when the generator phase hits the lower synchronisation boundary of $-0.3 \mathrm{~Hz}\left(-20^{\circ}\right)$. Respectively the upper boundary is the time when the generator phase passes by the synchronisation window with its upper limit $+0.3 \mathrm{~Hz}\left(+20^{\circ}\right)$ [109].

The phase increment for the simulation has been chosen to equal $30^{\circ}$, in order to speed up the simulation and still correctly calculate the synchronisation times throughout a $360^{\circ}$ cycle. When the increment equals $1^{\circ}$, the plot will be slightly different, this is depicted in Fig. 3.10. The vertical negative slope represents the time which is required for the control system to slip back a full cycle $(2 \pi)$, in order to find the next target grid cycle for synchronisation.

The exact synchronisation times could be obtained for various frequency 


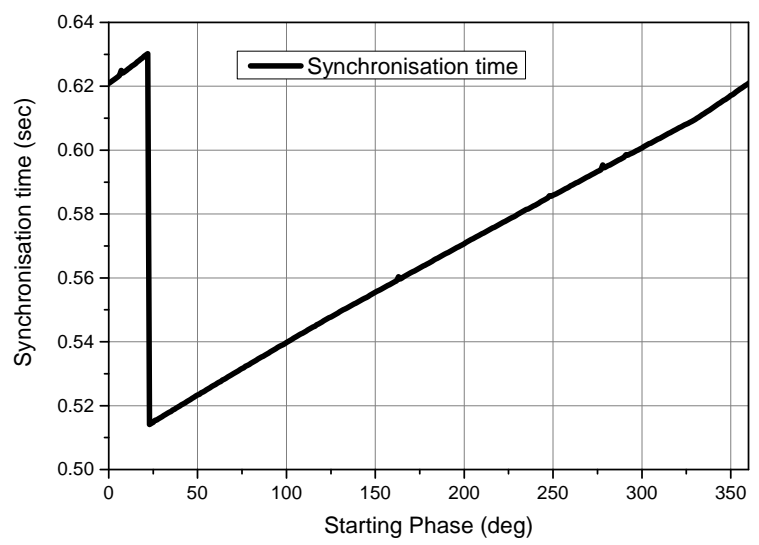

Figure 3.10: Simulation results of synchronisation time with the increment equals $1^{\circ}$

droop rates from Fig. 3.9 and plotted in Fig. 3.11 .

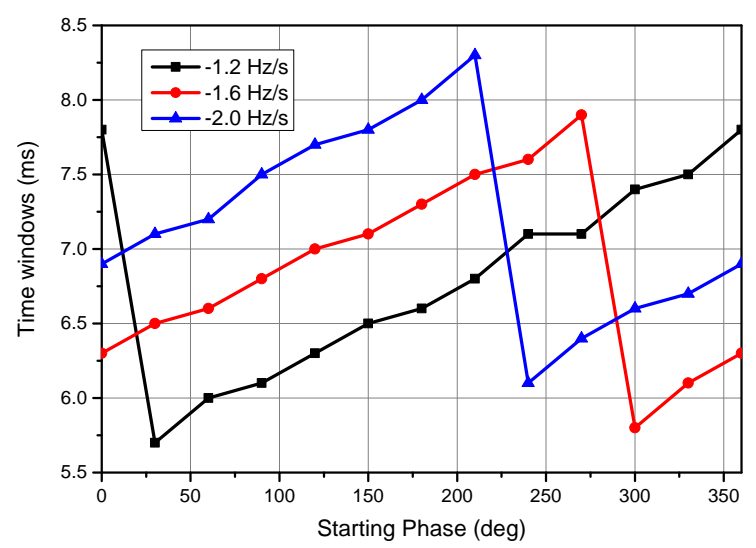

Figure 3.11: Time windows for the synchronisation moment with the range \pm $0.3 \mathrm{~Hz}$

However, in the ideal case shown above, this is assumed that connection to a power grid occurs instantaneously. In reality, delay times will be incurred through the use of mechanical contactors (typically $>100 \mathrm{~ms}$ ), and it is necessary for the hydraulic control to maintain synchronisation within the accepted window during this time. This information is vital for the depiction of the next plot, which represents the duration of these windows. They will help to understand how the control system should be modified (or not), in order to close a circuit breaker within the $\pm 0.3 \mathrm{~Hz}$ window. The plots are presented in Fig. 3.11

As it can be seen from the plot the longest time window is equal to 7.5 $8.5 \mathrm{~ms}$. This time frame is not enough for a circuit breaker to connect a syn- 


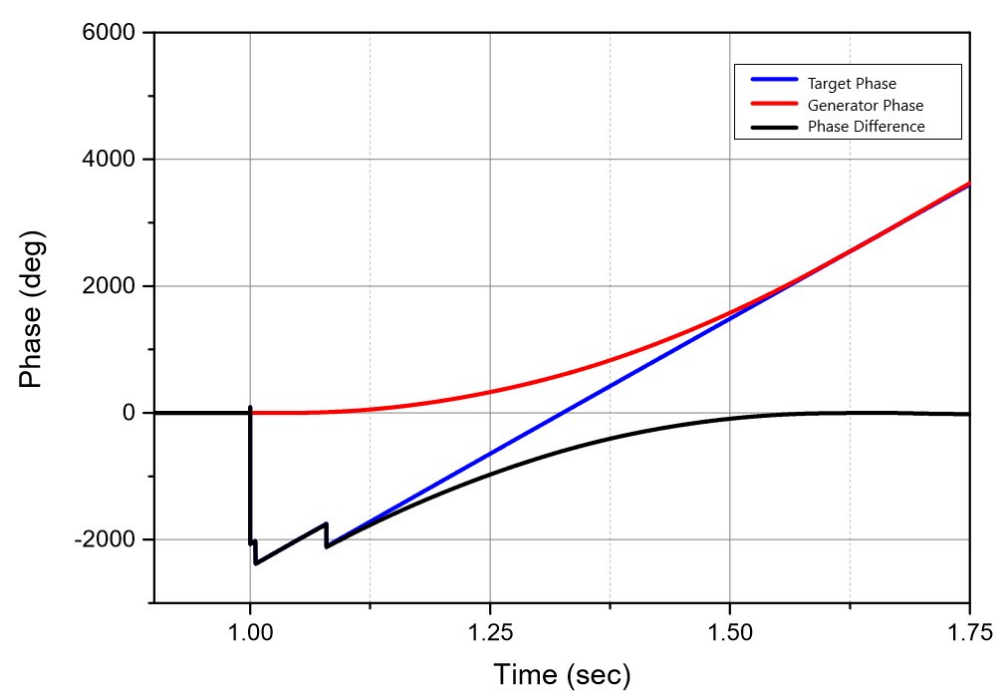

Figure 3.12: Simulink modelling results of the generator and the grid phases following an event to trigger rapid start-up and grid synchronisation by employing the modified computer model

chronous generator to a power grid. Meaning that it is imperative to modify the control system to extend the synchronisation windows to achieve stable synchronisation.

\subsubsection{Improved (2-stage) Ballistic Synchronisation approach}

In order to design a fully operational closed-loop system, which can be connected to the power grid during its acceleration, a 2-stage synchronisation approach was designed after the start of this $\mathrm{PhD}$ programme by the $\mathrm{PhD}$ author. Its phase profiles are depicted in Fig. 3.12 .

This mathematical model comprises a synchronous generator model, a standard Simulink excitation system, and a three-phase voltage source which is employed as an infinite bus for the connection to the synchronous generator.

Figure 3.12 depicts the modified (2-stage) ballistic synchronisation approach. It can be seen that the initial acceleration trajectory is the same as in Fig. 3.8. however at the synchronisation moment the novel approach enters a gridfollowing mode. It enables the generator to track the phase and the frequency (velocity) of the power grid to maintain the generator within the synchronisation boundaries.

By employing the modified model, it is possible to depict the graphs representing grid and generator voltage, since their behaviour is one of the requirements for the successful synchronisation of the generator with a power grid.

The plots are presented in Fig. 3.13 and depict the zoomed positive mag- 
nitudes of the sinusoidal voltage waveforms (only phase $\mathrm{C}$ is shown). The

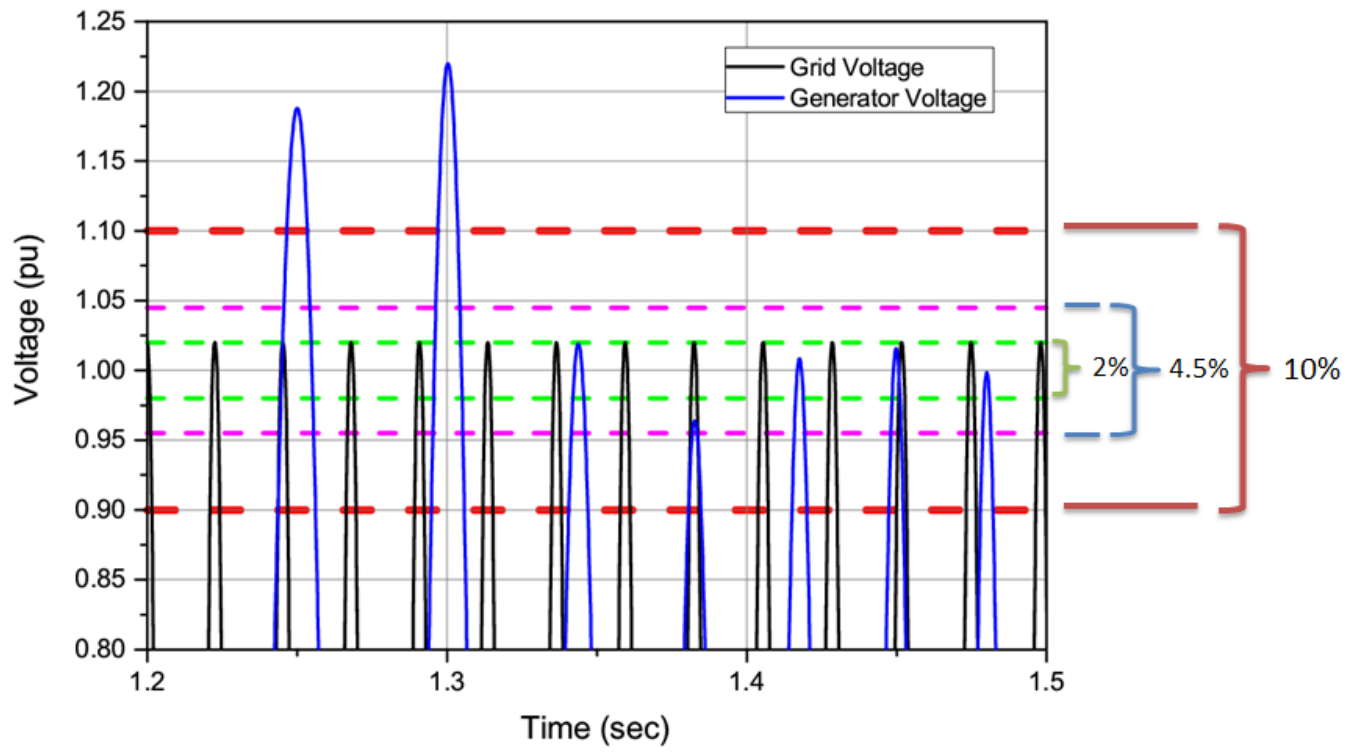

Figure 3.13: Simulation results of the generator and grid voltage with the acceptable boundaries for synchronisation (triggered at 1s).

horizontal lines represent different synchronisation boundaries for the voltage requirements based on the manufacturer's data for generator ABB AMG0250. The recommended voltage difference between the generator and the grid equals $2 \%$ (1.02 - $0.98 \mathrm{pu})$, whilst $4.5 \%$ (1.045 - $0.955 \mathrm{pu})$ is its maximum value. The voltage difference of $10 \%$ is shown in Fig. 3.13 according to the IEEE 1547 Standard [109].

It could be seen that even in the worst case scenario (where the frequency droop rate is $-2 \mathrm{~Hz} / \mathrm{s}$ ), the voltage error is within the acceptable range after $0.3-0.4 \mathrm{~s}$ from the moment when the system is triggered. This is the evidence that the voltage equality between the generator and the grid is reached before the frequency and phase angles match each other.

\subsection{Chapter Summary}

In this chapter, a mathematical description of the ballistic and novel proposed 2-stage ballistic synchronisation approach was given, supported by the presented simulation results for both of the methods. The chapter then presented an overview of the employed ss-CAES systems that were tested for the synchronisation approach verification. 


\section{Chapter 4}

\section{Hardware for ss-CAES Prototype Experimentation}

This chapter focuses on a description of the built hardware prototype and further steps for the experimental verification of the synchronisation method.

\subsection{Hardware for Experimental Testing of the pro- totype}

Chapter 3 verified the proposed synchronisation approach. However, in order to deliver a working hardware prototype, it is required to verify the main components of the hardware prototype, update the model, and then compare the simulated outputs with the experimental results.

The hardware prototype was built through research funding from the New Zealand Ministry of Business, Innovation, and Employment. It was designed to fit within a standard $20 \mathrm{ft}$ shipping container which provides both transport and a safety enclosure for the hydraulic machinery. The prototype was assembled before the start of the PhD program, and further design alterations have occurred since then. Its 3D model is shown in Fig. 4.1, its block diagram is depicted in Fig. 4.2 and the pictures of the assembled system are presented in Fig. 4.3.

The primary data acquisition set up was designed in the Labview environment with the use of the Signal Processing toolkit. However, for the implementation of the primary acceleration derivation code, another toolkit has been utilised - Mathscript RT. The main reason is that the acceleration code was written in MATLAB and then used in the main mathematical model in the Simulink/Simscape environment. Hence the use of the same MATLAB code could drastically reduce the time for the hardware implementation [71, 110]. In 


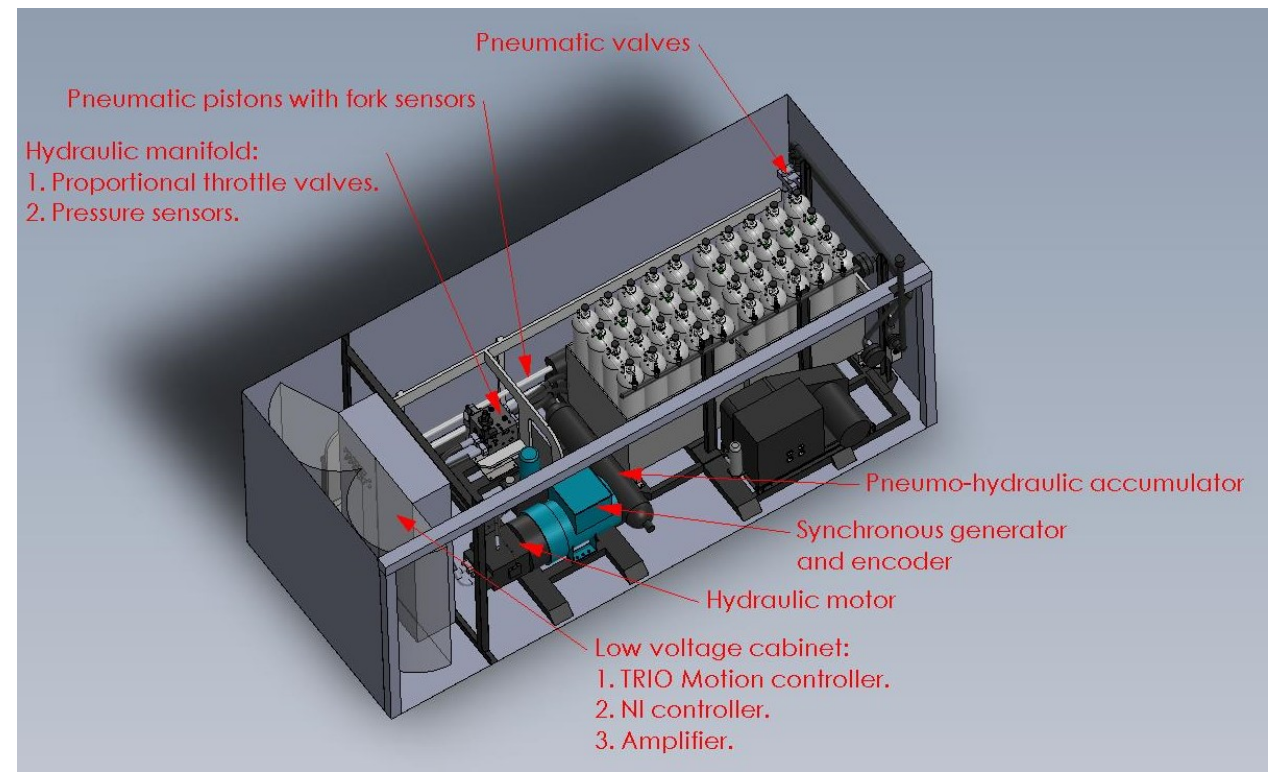

Figure 4.1: A three dimensional model of the ss-CAES prototype.

most cases, this toolkit is used with the Control and Simulation toolkit, which mimics Simulink. There are few Mathscript applications with the use of standard Labview libraries. Even though it is a powerful library that bridges mathematical modelling in MATLAB/Simulink and hardware verification in Labview, there are not many researchers who can use this tool effectively.

There are various limitations of this toolkit that can be found on the official National Instruments website. It should be noted that the main acceleration derivation code was implemented without any unsupported functions or libraries. However, it should be specified that the toolkit does not precisely like the MATLAB environment. During the preparation for the experimental testing, it was noticed that the main script with if-cycles does not output the same results as it does in MATLAB. Labview executes the whole script from line 1, even though the requirements for if-cycles have been fulfilled. Whereas MATLAB will stay in the if-cycle while the if condition is fulfilled. Hence it is required to change the scripts so their outputs will match with the corresponding MATLAB scripts.

\subsubsection{DAQ system}

The data acquisition system for the components testing was designed by the $\mathrm{PhD}$ author with the use of a National Instruments controller cRIO-9068 with the following input/output modules and components - Fig. 4.4 


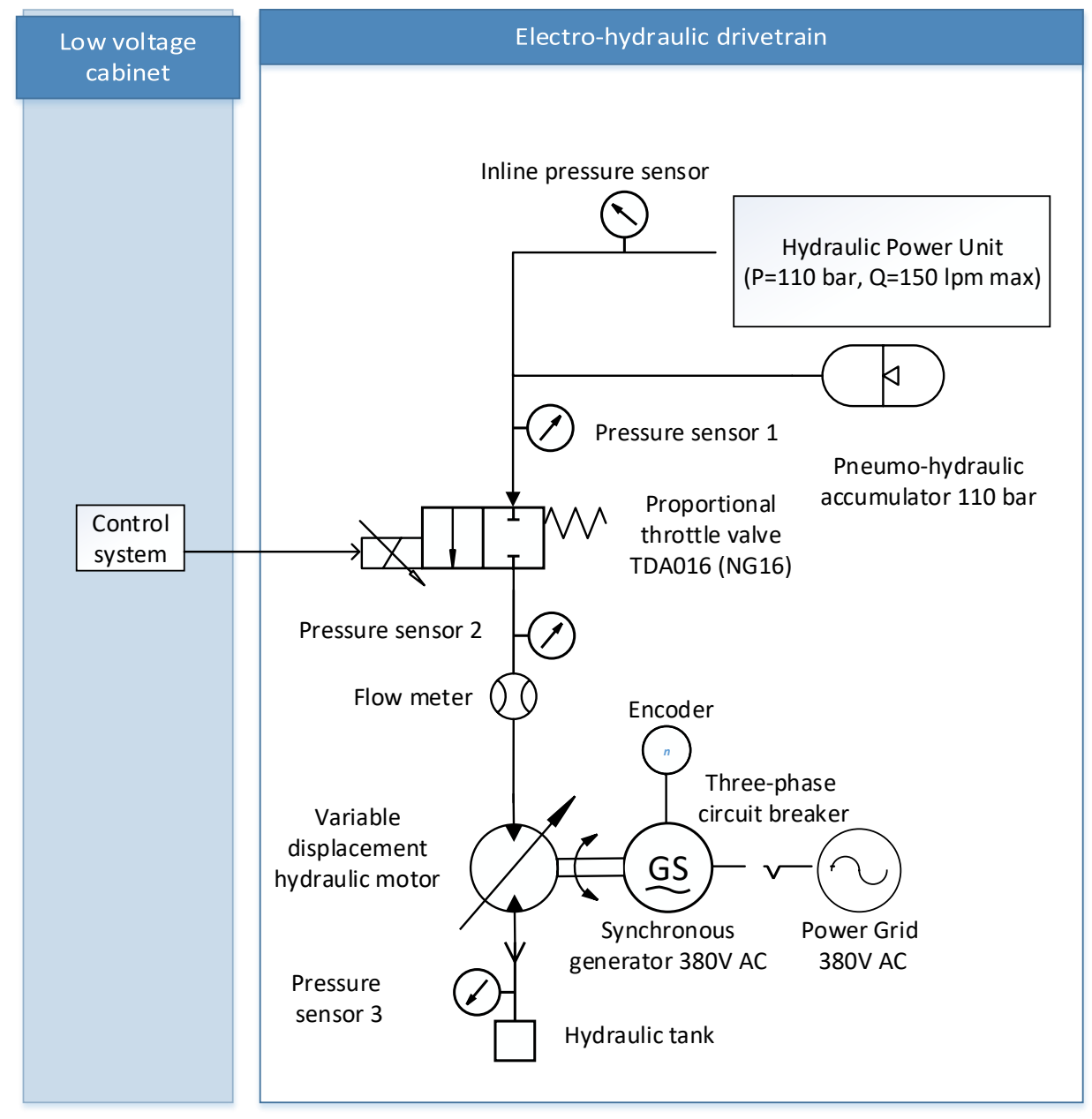

Figure 4.2: Schematic of the main components of the prototype. 


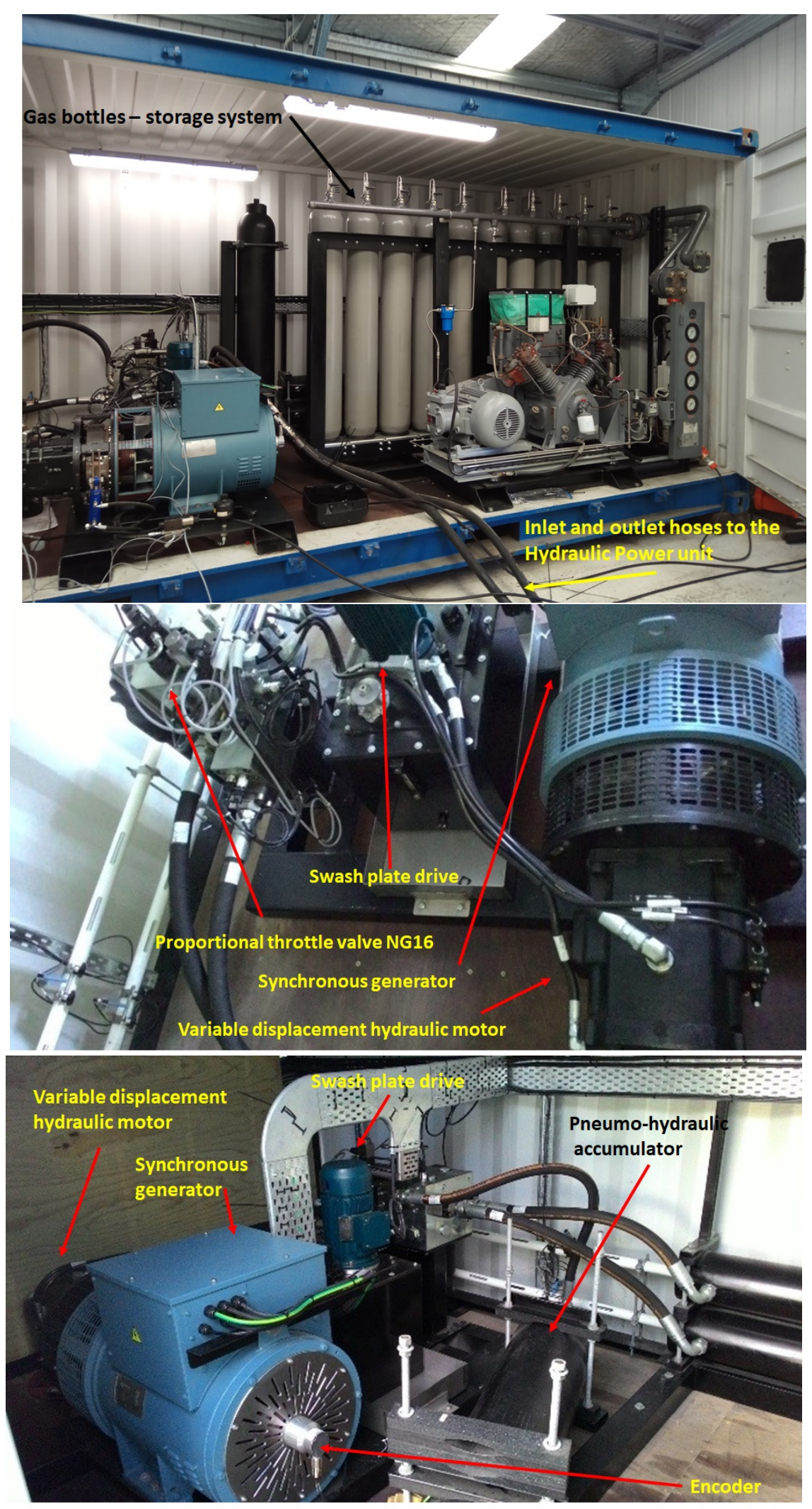

Figure 4.3: Small Scale Compressed Air Energy Storage system during the final stage of its assembling. 


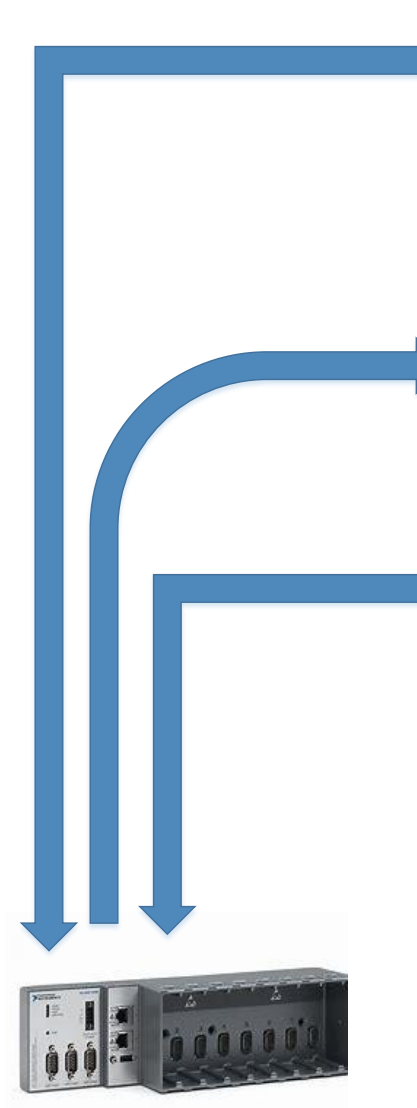

NI cRIO 9068

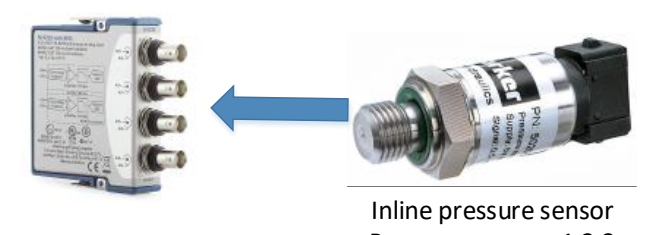

$\mathrm{N} 19229 \mathrm{Al}+/-60 \mathrm{~V}$

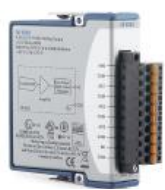

$\mathrm{N} 19263 \mathrm{AO}+/-$

$10 \mathrm{~V}$

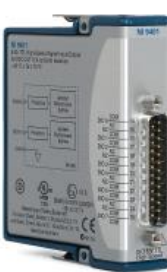

NI9401 DI

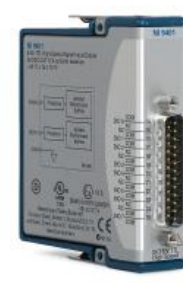

NI9401 D
Pressure sensors 1,2,3

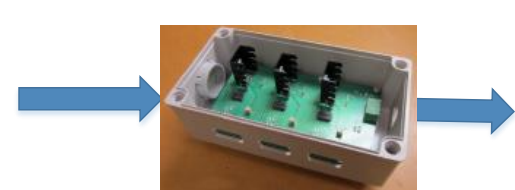

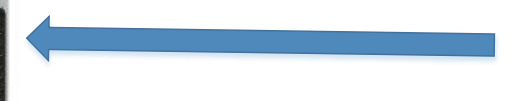

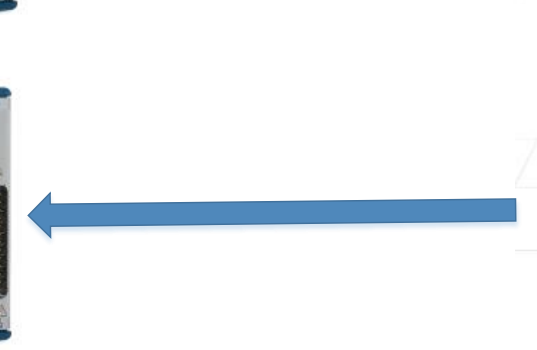

(1)

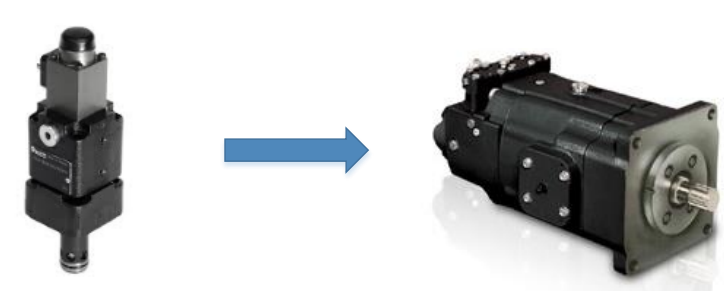

Control valve NG16

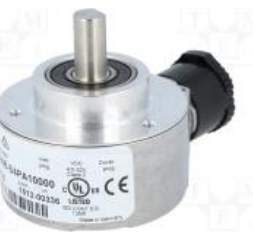

Quadrature optical encoder

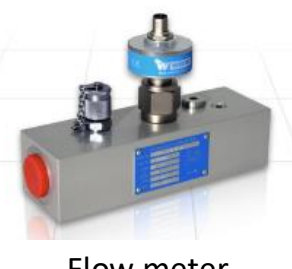

Flow meter hydraulic motor M14
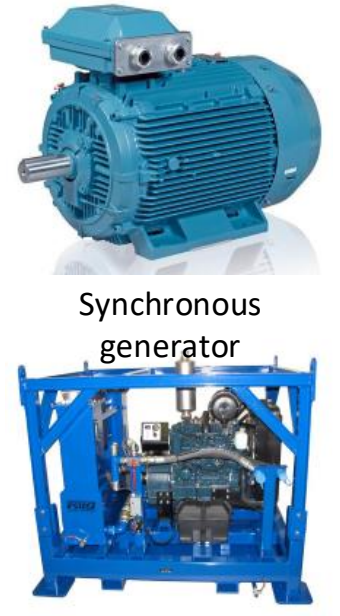

Figure 4.4: Data acquisition system for experimental validation of the described in the research prototype.

Hydraulic power unit 
With the fully assembled hardware prototype and the data acquisition system, it is possible to start the verification process of the main components of the system. It leads to the updated mathematical model that will verify the performance of the experimental setup. The prototype has two main parts - the low voltage cabinet and the electro-hydraulic drivetrain. They are discussed later in this section.

\subsubsection{Low voltage controller cabinet}

The prototype's low voltage controller cabinet is shown in Fig. 4.5. Its wiring was completed in the beginning of this PhD project by the PhD author. The following components are placed in the cabinet:

1. National Instruments controller NI-9068.

2. TRIO Motion controller MC464.

3. Amplifier.

1. National Instruments controller. A NI controller cRIO-9068 is the central controller of the ss-CAES prototype. This is a $667 \mathrm{MHz}$ Dual-Core CPU, 512 MB DRAM, 1 GB Storage, Zynq-7020 FPGA, Extended Temperature, 8-Slot CompactRIO Controller. It features an FPGA and a processor running NI Linux OS. To expand its functionality, the following analog and digital modules were utilised:

- NI 9063 Analog output module. It's a 100 kS/s/ch Simultaneous, $\pm 10 \mathrm{~V}$, 4-Channel C Series Voltage Output Module. This module controls the amplifier to drive the proportional throttle valves.

- NI 9225 Analog input module. It has a voltage range of $\pm 60 \mathrm{~V}$ with the maximum sample rate of $50 \mathrm{kS} / \mathrm{s} / \mathrm{ch}$. The module inputs the output signals from the pressure sensors.

- NI 9401 Digital module. It's a 5 V/TTL, 8 Bidirectional Channels, 100 ns C Series Digital Module that inputs the encoder and flowmeter output signals.

2. TRIO motion controller. The TRIO motion controller MC464 was initially implemented to monitor the system's pressure, temperature sensors, to control the hydraulic drum brake and the pneumatic valves at the cylinders by inputting the outputs from the fork sensors. The controller can 


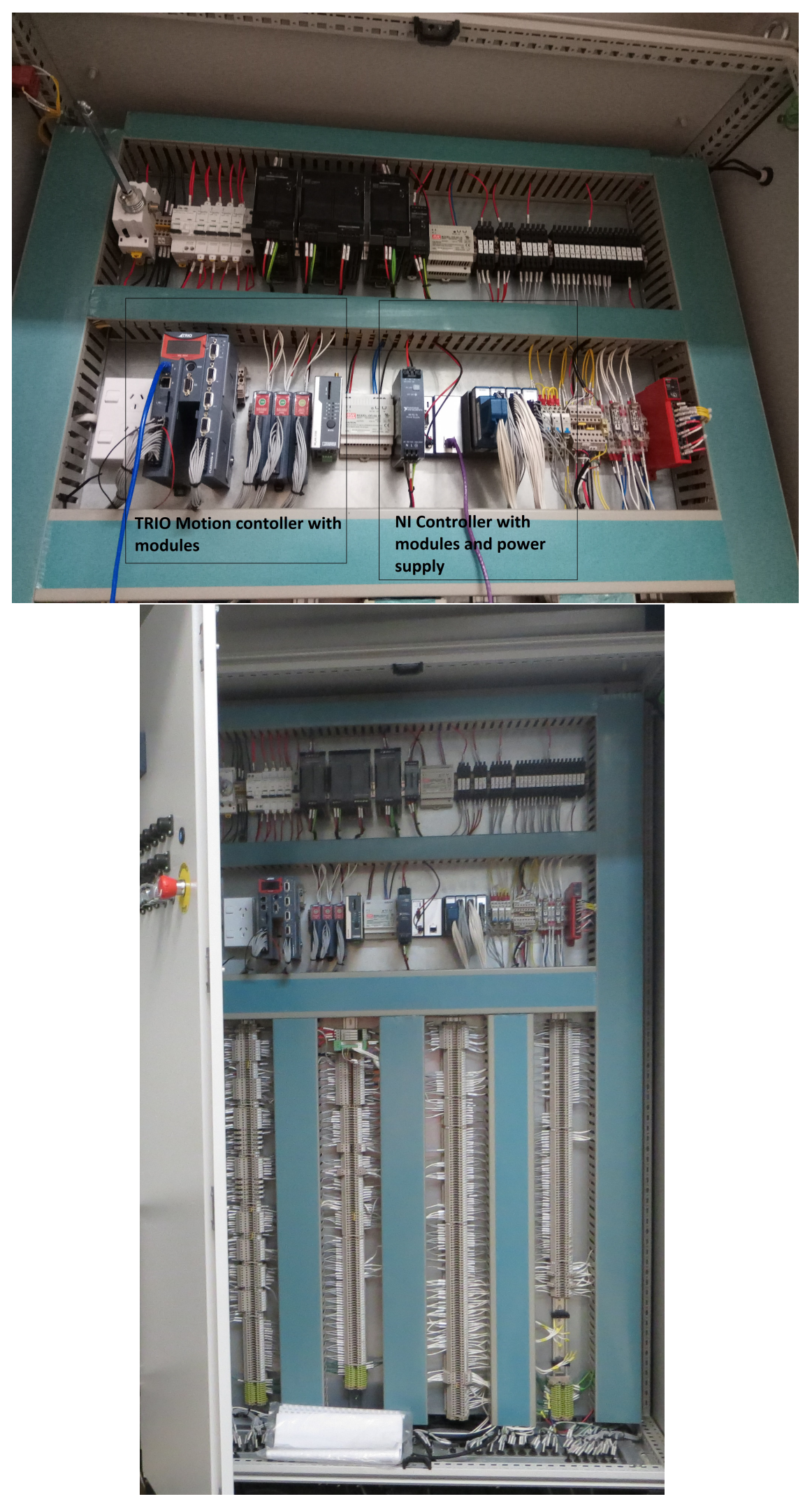

Figure 4.5: Low voltage cabinet of the ss-CAES prototype. 
be programmed with use of one of the five official IEC 61131-3 programming languages. For the synchronisation technique testing this controller did not operate due to the necessity to acquire all the input and output signals via one controller (NI controller). The controller with its modules is presented in Fig. 4.6 .

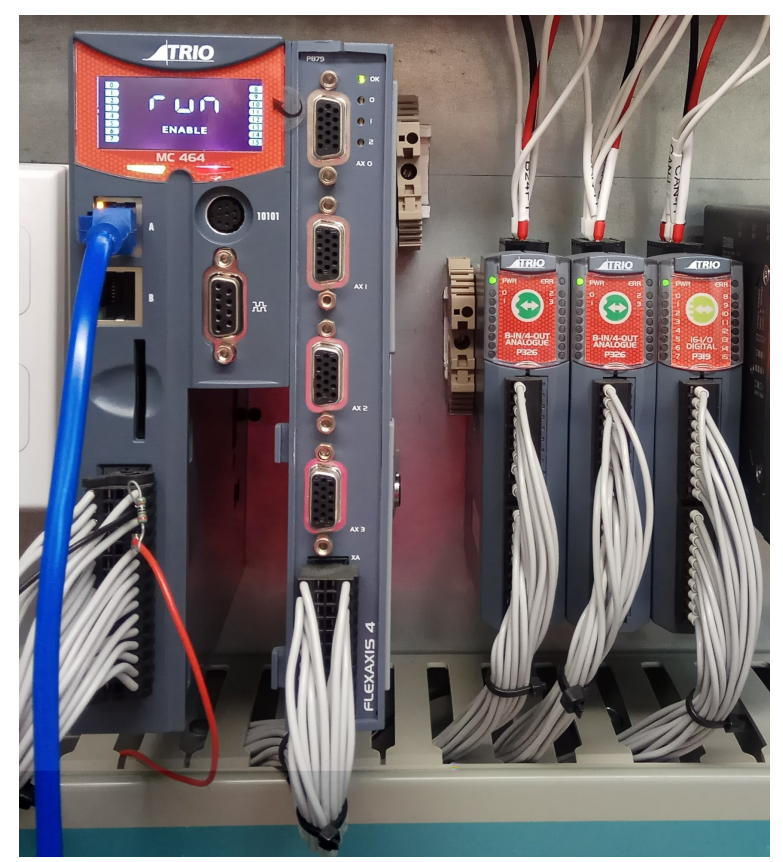

Figure 4.6: TRIO Motion controller MC464 with two analog modules P326 and a digital module P319 in the low voltage cabinet of the ss-CAES prototype.

3. Amplifier. To fully control the current driven proportional throttle valve (TDA016) employed in the prototype, it is required to deliver the maximum current of $1.05 \mathrm{~A}$ at $16 \mathrm{VDC}$. The controller utilised for the testing, NI cRIO-9068, with the output module NI9263 can deliver +/- 10 VDC and $20 \mathrm{~mA}$. Hence, to operate the valve, an amplifier has been designed to provide the required opening current (voltage) (shown in Fig. 4.7 and Fig. 4.8). A voltage output from the controller controls a current output from the amplifier, which creates a current loop through the valve's coil to energise it. It is a three channel amplifier and one of the channels is shown in Fig. 4.9. It should be noted that there are no current output National Instruments modules that can deliver the output current up to 1.05 A directly.

The amplifier works by using negative feedback. The current taken by the load is sensed by the current sensing resistor (R2), and the op-amp adjusts the current so that the output current is always Vin/R2. The output of the NI9263 is connected to the op-amp through a trimpot R1. It 


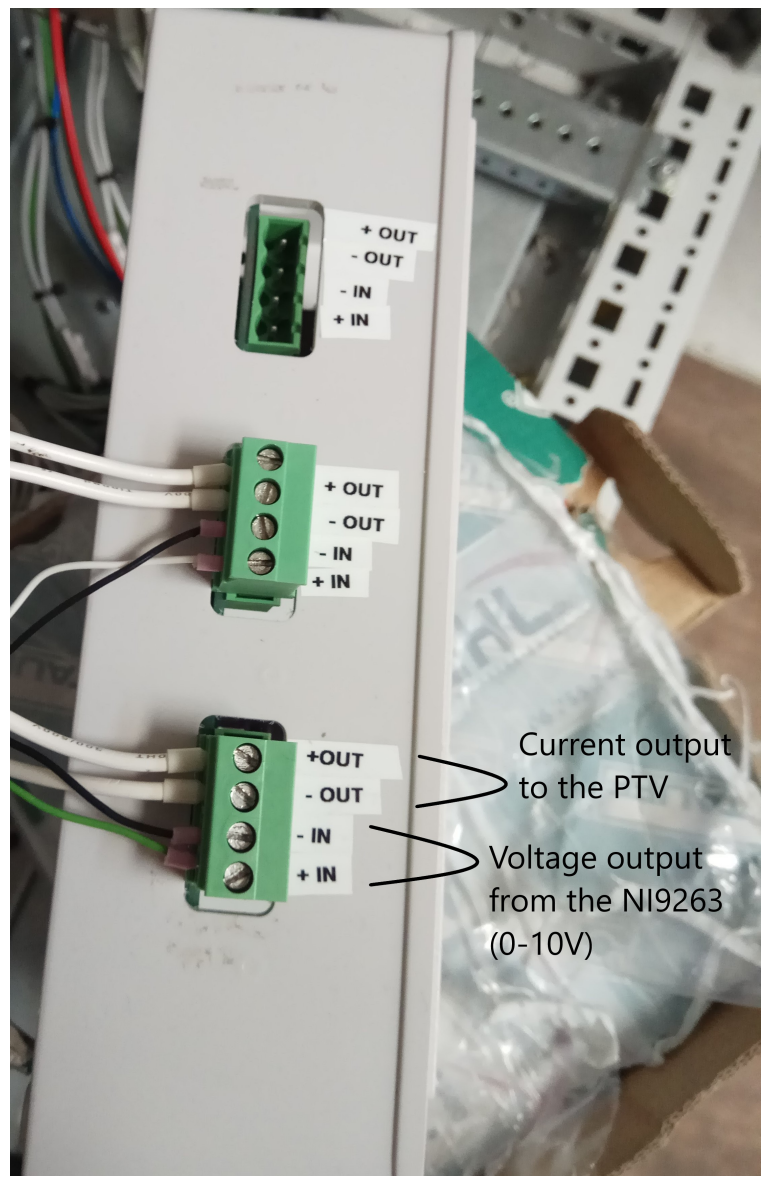

Figure 4.7: Input and output terminals of the amplifier.

allows adjusting the output current by changing the Vin. The value of the R1 needs to be high enough not to overload the output stage of the NI9263. In our case, it is $10 \mathrm{~K}$.

The current sensing resistor $\mathrm{R} 2$ is a $1 \mathrm{Ohm}, 2 \mathrm{~W}$ power metal strip resistor. The op-amp, OPA548, is a low-cost, high-voltage/high-current operational amplifier with thermal shutdown and adjustable current limit. It can deliver 3A continuous output current with supply range up to +/30V. The current limiting resistor R3 sets the current limit to approximately 3 Amps. The internal thermal shutdown will disconnect the output when the temperature of the die reaches 1600C. Additionally, the op-amp is protected with resettable PTC fuses (F1, F2,) in power supply lines. The op-amp is in the TO-220 package and attached to a heat sink with fan assisted cooling. In order to drive the amplifier, a $20 \mathrm{~V}$ power supply has been designed. It was required due to the necessity of having the amplifier's output current in the range from 0 to stable 1.05A.

For experimental testing in New Plymouth, the amplifier was connected as it is shown in Fig. 4.10, according to the following procedure: 


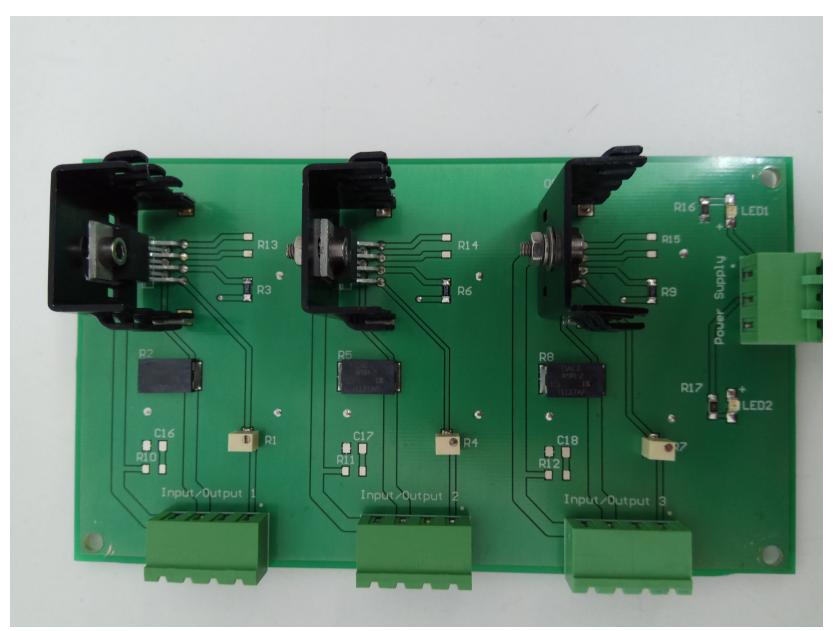

Figure 4.8: The PCB of the designed amplifier.

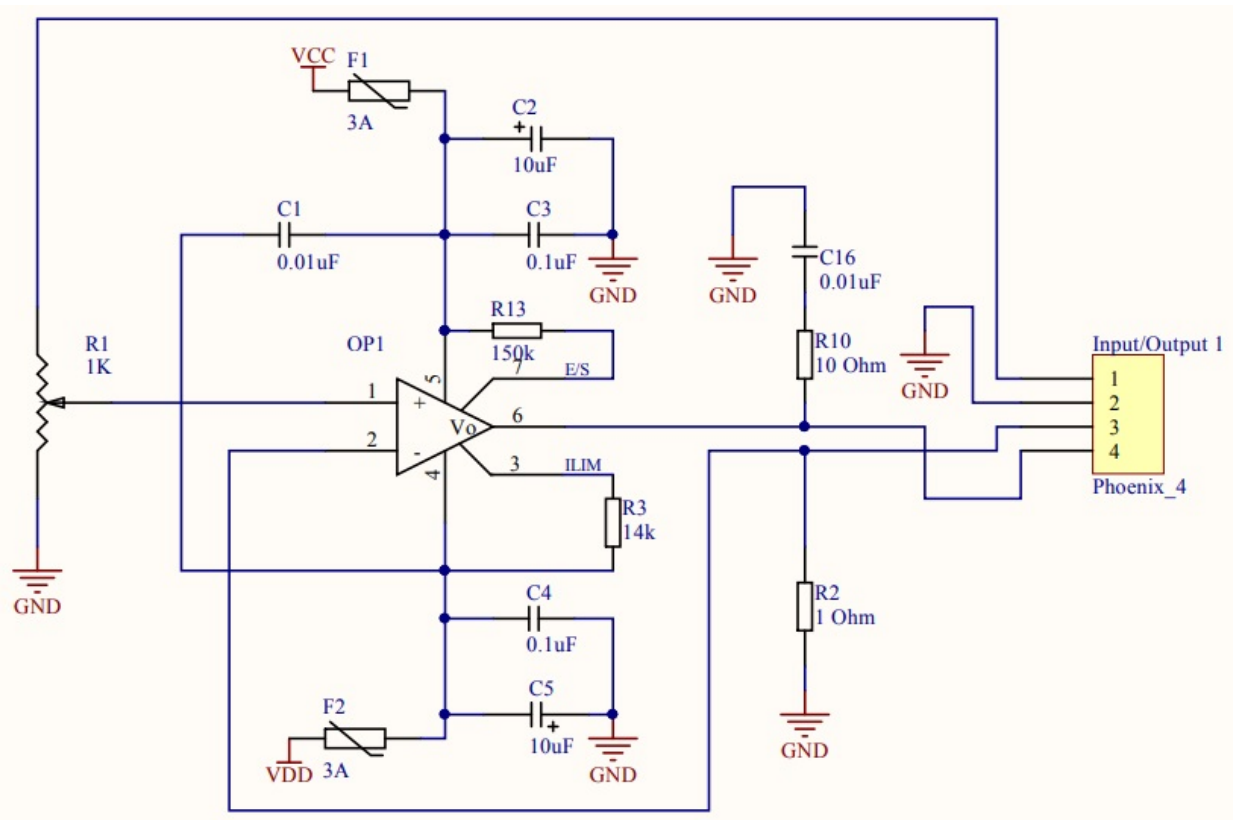

Figure 4.9: Output channel 1 of the designed amplifier.

(a) Connect two common wires (green and yellow) to the power supply inputs Output 1+ and Output 2-. In this case, -VCC is connected to the negative Output 1- and +VCC to the positive Output 2+. It will give the required positive and negative voltage for the amplifier.

(b) Set up the power supply's voltage to $16 \mathrm{~V}$.

(c) Connect IN- and IN+ to the NI9263 (AO0). OUT- and OUT+ are connected to the valve coil. By controlling the AO0 voltage via Labview, it is possible to control the opening of the valve. However, the main aspect is a control current flowing through the PTV coil. It is necessary to verify the current when the amplifier is connected to the PTV. 


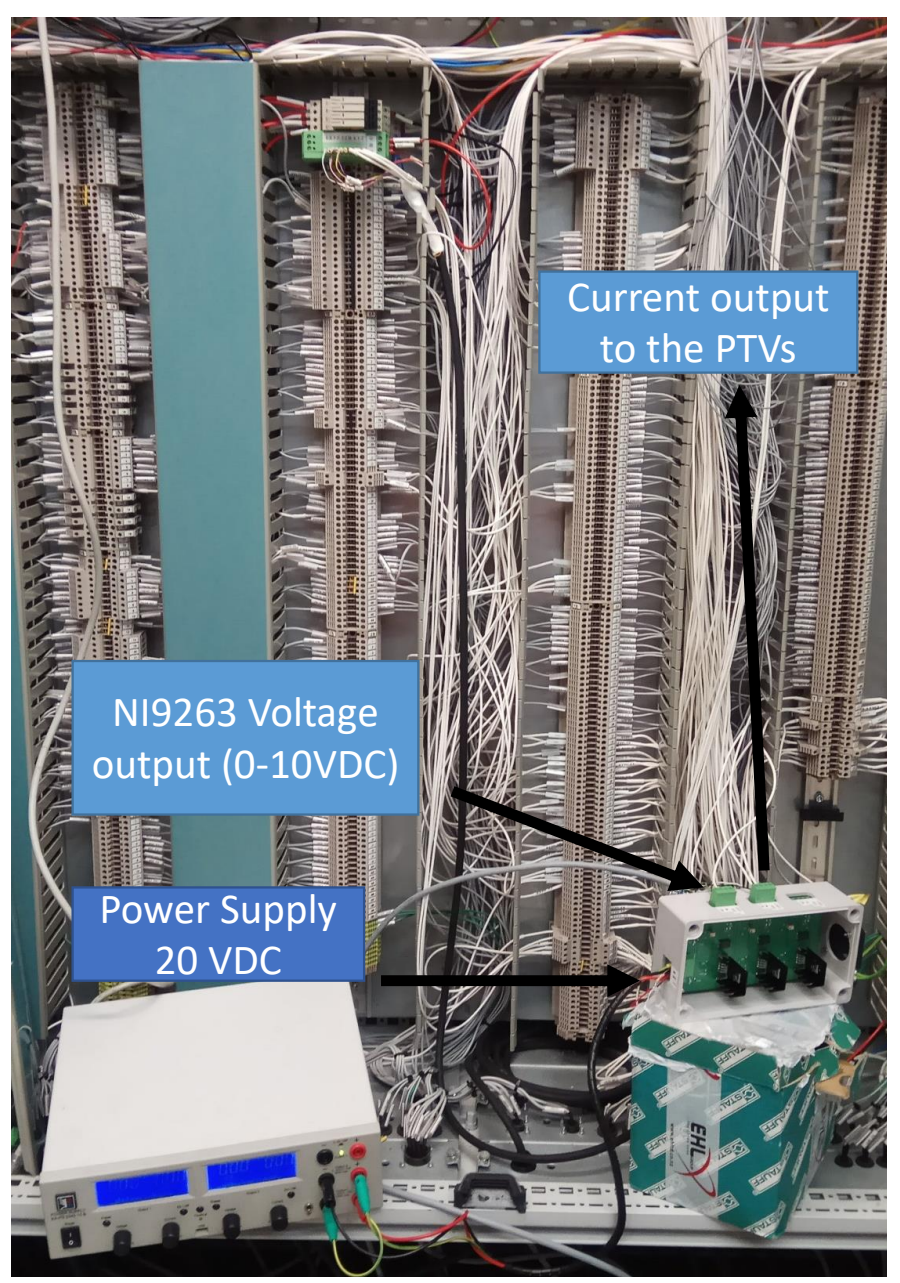

Figure 4.10: Amplifier is connected in the low voltage cabinet during experimental testing.

(d) By gradually increasing the control voltage (AO0) measure the current though $\mathrm{IN}+$ connection by using a multimeter.

(e) Record the voltage values with the corresponding current values. Ensure that the maximum value of the current is at least $1.05 \mathrm{~A}$. It is required to fully open an TDA016 valve.

The first runs of the programmed NI controller did not open or change the orifice areas (openings) of the valves. The troubleshooting showed that the resistance of the valve's current loops was too low. According to the technical specifications of the NI9263, the minimum resistance of a circuit connected to the module should be at least $10 \mathrm{kOhm}$. To accommodate this requirement, additional resistors were put into the amplifier's channels. The amplifier also got upgraded heat sinks to prolong the life of the device. Before retrofitting the amplifier, it was tested by recording its voltage-current relationship using a test rig at Robinson Re- 
Table 4.1: Voltage-current testing of the amplifier

\begin{tabular}{|l|l|l|l|l|l|l|l|l|l|l|l|l|l|l|l|l|l|}
\hline & \multicolumn{11}{|c|}{ Voltage (V) } \\
\hline & 2 & 2.5 & 3 & 3.5 & 4 & 4.5 & 5 & 5.5 & 6 & 6.5 & 7 & 7.5 & 8 & 8.5 & 9 & 9.5 & 10 \\
\hline Second Channel Current (A) & 0.18 & 0.24 & 0.29 & 0.35 & 0.4 & 0.46 & 0.51 & 0.56 & 0.62 & 0.67 & 0.73 & 0.78 & 0.84 & 0.89 & 0.95 & 1 & 1.05 \\
\hline Third Channel Current (A) & 0.19 & 0.24 & 0.3 & 0.35 & 0.4 & 0.46 & 0.52 & 0.57 & 0.63 & 0.68 & 0.73 & 0.79 & 0.84 & 0.9 & 0.95 & 1.01 & 1.05 \\
\hline
\end{tabular}

search Institute. This data is presented in Table. 3.3. It should be noted that the first channel of the amplifier was damaged due overheating at the first experimental run of the prototype in New Plymouth and was not used during further tests.

The valves open at the voltage of 3.4-3.5V regardless of the employed amplifier's channel. Channel Three was used as a primary channel for controlling the NG16, whereas the Channel Two was controlling the valve installed in the manifold (fully open all the time).

\subsubsection{Electro-hydraulic drivetrain}

The electro-hydraulic drivetrain controlled by the aforementioned control system has the following components:

- Synchronous generator. It is a 4-pole synchronous machine with a Baisler excitation system. The generator was chosen and installed before the start of this PhD project. In case of a contingent event in the power grid, the generator is accelerated and synchronised with the power grid. Manufactured by $\mathrm{ABB}$ with the power rating output of $100 \mathrm{~kW}, 400 \mathrm{~V} / 230 \mathrm{~V}$, $50 \mathrm{~Hz}$. The most financially feasible option is to use off-the-shelf components in the system's design. Taking into account that the main synchronisation algorithm controls the dynamics of the generator, it is imperative to highlight the difference between electrical and mechanical parameters of the generator.

Firstly it is required to show a relationship between the electrical frequency of the generator and its mechanical velocity (rotor velocity).

$$
f_{\text {elec }}=n_{\text {mech }} \cdot N_{p p}
$$

where $f_{\text {elec }}$ is the electrical frequency of the generator in $\mathrm{Hz}, n_{\text {mech }}$ is the mechanical velocity of the generator in rev/s and $N_{p p}$ is the number of the rotor pole pairs. In the same manner the generator position can be shown as:

$$
\theta_{\text {elec }}=\theta_{\text {mech }} \cdot N_{p p}
$$


where $\theta_{\text {elec }}$ and $\theta_{\text {mech }}$ are the electrical and mechanical phase (position) of the generator in radians. The electrical power output can be found as:

$$
P_{\text {elec }}=\tau \cdot \omega_{\text {mech }}
$$

where $P_{\text {elec }}$ is the electrical power generated by the generator $(\mathrm{W}), \tau$ is the mechanical torque produced by the generator in $N \cdot m$ and $\omega_{\text {mech }}$ is the mechanical speed in $\mathrm{rad} / \mathrm{s}$.

The maximum torque of the generator can be found as:

$$
\tau_{\text {max }}=\frac{P_{\text {elec }}}{\omega_{\text {mech }}}=\frac{100 \cdot 10^{3}}{25 \cdot 2 \pi}=636 \mathrm{Nm}
$$

- A pneumohydraulic accumulator is the primary source of energy in the presented hardware prototype. It was chosen and installed before the start of this PhD project. These devices store energy in the form of compressed gas and separating elements. In case of the ss-CAES, its accumulator is precharged with nitrogen (industrial standard) and uses a bladder for separation of the gas and the hydraulic fluid inside the device. It is pressurised up to 350 bar (at the capacity of 501 ) and capable of providing sufficient energy to the electro-hydraulic drivetrain. When the system is triggered, and the accumulator is being discharged, its pressure drops as well. However, for the acceleration stage of the generator studied here, the input pressure is assumed to be constant.

- A $134 \mathrm{HP}$ variable displacement hydraulic motor Gold Cup M14 is coupled to the generator to accelerate it. It was chosen and installed before the start of this PhD project. To control the torque transmitted to the generator, it is required to adjust the motor's displacement by utilising the swash-plate drive mechanism. During the system's operation the accumulator's output pressure decreases, as well as the flow rate. Since the motor's velocity can be calculated as:

$$
N=\frac{Q}{V_{D}}
$$

where $N$ is the velocity of the motor in RPM, $Q$ is the flow rate in the hydraulic circuit (lpm), and $V_{D}$ is the displacement of the motor $(1 / \mathrm{rev})$. When the flow rate decreases, the velocity of the motor can be kept constant only by changing the displacement of the motor.

Three efficiencies are employed to characterise a hydraulic motor named overall, mechanical and volumetric efficiency. The mechanical efficiency is a correlation between the theoretical output torque and the real torque. 
The volumetric efficiency at the same time describes the relationship between the theoretical and actual flow rate through the motor.

Since the main application of the hydraulic motor is a fast accelerated hydraulic drivetrain, it is imperative to calculate the maximum achievable acceleration of the motor:

$$
\begin{gathered}
\alpha_{\text {theor }}=\frac{\tau_{\text {nom }}}{J_{\text {motor }}+J_{\text {gen }}}=\frac{636.6}{1.45+0.0085}=414.7 \mathrm{rad} / \mathrm{s}^{2} \\
t_{\text {sync }}=\frac{\omega_{\text {sync }}}{\alpha_{\text {theor }}}=0.38 \mathrm{~s}
\end{gathered}
$$

where $\alpha_{\text {theor }}$ is the theoretical acceleration of the drivetrain $\left(\mathrm{rad} / \mathrm{s}^{2}\right), \tau_{\text {nim }}$ is the nominal torque of the hydraulic motor $(N \cdot m), J_{m o t o r}$ is the inertia of the motor $\left(\mathrm{kg} \cdot \mathrm{m}^{2}\right), J_{g e n}$ is the inertia of the synchronous generator $\left(\mathrm{kg} \cdot \mathrm{m}^{2}\right), t_{\text {sync }}$ is the synchronisation time (s), $\omega_{\text {sync }}$ is the synchronisation speed in $\mathrm{rad} / \mathrm{s}$. It should be mentioned that the derived synchronisation time is theoretical and is different from the real values due to the combined inertia of the electro hydraulic drivetrain.

- Proportional throttle valve TDA016. The valve was chosen and installed as part of the current $\mathrm{PhD}$ project. The central actuator in the hydraulic circuit is a proportional throttle valve TDA016, which controls a flow through the hydraulic motor. To achieve the maximum flexibility of the drive train dynamics, two proportional valves have been employed. During the system's testing one of theses valves was fully opened, due to the inadequate performance of the inline valve. This valve is depicted in Fig. 4.11 and it is mounted on top of the hydraulic manifold. It is a Parker TDA016 current driver valve with a 16 VDC coil. In the case of this valve it is required to achieve the coil current of $1.05 \mathrm{~A}$ to fully open it. Since the valve (valves) are controlled by the NI controller, it is necessary to output a current from it up to 1.05A. There are no current external modules (Cseries) that would be able to achieve such high values. To overcome this issue, an amplifier has been designed by the electrical engineering team of Robinson Research Institute.

A hysteresis effect in operation of the proportional throttle valve takes place when the control current increases and then decreases. In this case, due to the saturation of the coil, the flow rate at the current flowing down is higher than the current flowing up. Meaning that for stable control of the electro-hydraulic drivetrain, a hysteresis compensation algorithm should be implemented. 


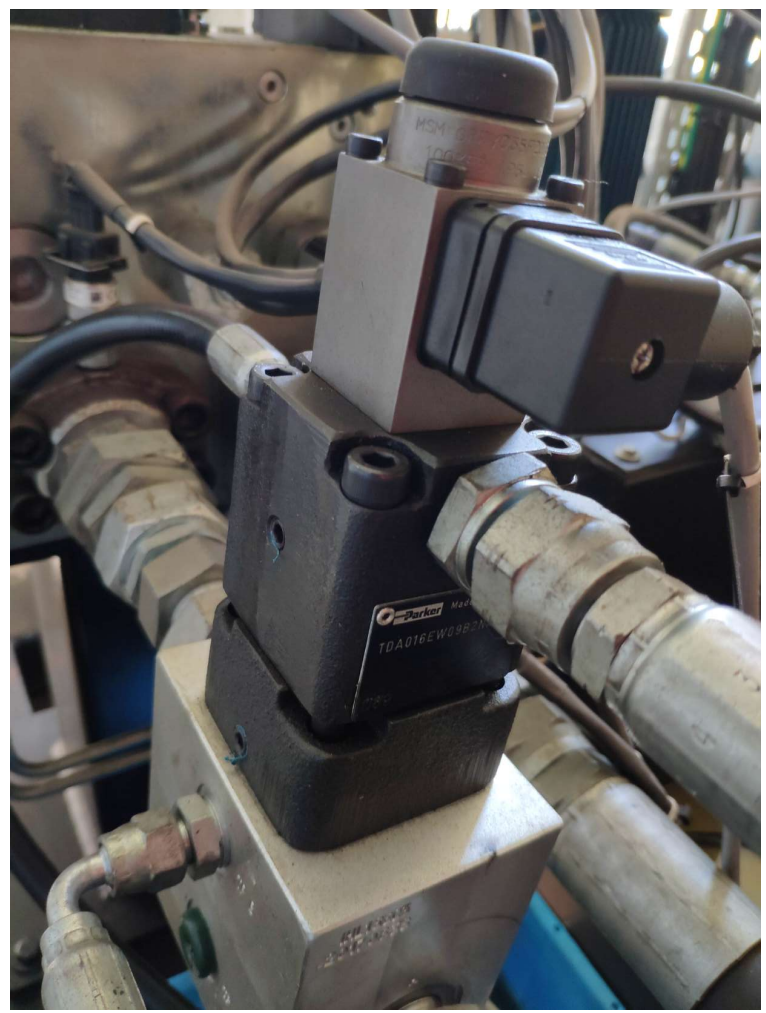

Figure 4.11: TDA016 Proportional throttle valve employed in the ss-CAES prototype.

- Pressure sensors. The sensors that are employed in the system are manufactured by Parker (IQAN-SP) (shown in Fig. 4.12) and have the following specifications:

- Pressure range 0 - 500 bar $(0.5-5 \mathrm{~V})$.

- Burst pressure 1500 bar.

- Type B with a G1/4 thread.

- Integral 3 pin connector is a sealed AMP Junior type.

All of the sensors are connected to the NI-9229 analogue input module installed on the cRIO board. They continually monitor the inline pressure (referring to Fig. 4.2) from the hydraulic power unit (inline pressure sensor) and the differential pressure profiles across the valve (pressure sensor 1 - pressure sensor 2) and the hydraulic motor (pressure sensor 2 - pressure sensor 3). In case of the testing with the input from the accumulator, the inline pressure sensor can be placed into the accumulator output circuit. It is an important safety feature to ensure that the pressure in the hydraulic circuit will not exceed the limit of 350bar. 


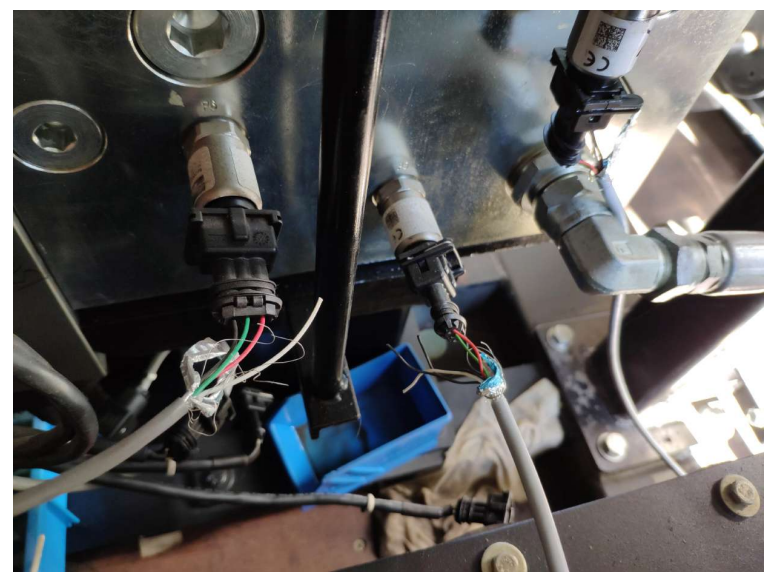

Figure 4.12: Pressure sensors IQAN-SP employed in the ss-CAES prototype (located on the manifold).

The pressure sensor measurements are saved via a logging loop utilising the standard Labview tdms tools. Meanwhile, the same pressure readings are also passed to a differential pressure loop. It is specifically designed to find a moment when the differential pressure reaches the required 50/70/100 bar (can be modified online via the central NI controller). The differential pressure loop compares the difference between the sensors readings and the specified differential pressure value. As soon as the required pressure is reached, the "Differential" indicator goes off on the front panel. This indicates the moment when the flow rate measurements can be recorded. All the sensors are connected to the NI-9229 Analogue input module installed in the low voltage cabinet.

- Incremental encoder. The incremental encoder installed on the generator's shaft monitors the position, velocity and acceleration profiles of the electro-hydraulic drivetrain. The quadrature encoder DFS60B-S4PA10000 - manufactured by Sick - can provide high-resolution measurements up to 16 bit (up to 10000 pulses per revolution) with the accuracy of \pm 0.008 . The output frequency is less than $600 \mathrm{kHz}$, and the signal from it is measured by the NI-9401 module installed on board the controller chassis. The installed in the prototype encoder is shown in Fig. 4.13

- Pulses per revolution - 10000 (40000 in X4 mode).

- 4.5...32 V, TTL interface.

- Output frequency - <600 kHz. 


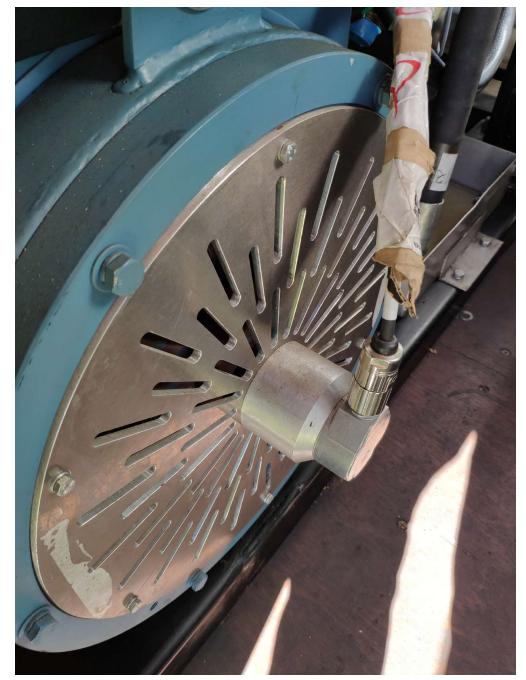

Figure 4.13: SICK DFS60 incremental encoder utilised for measuring the dynamics of the electro-hydraulic drivetrain.

Flow meter turbine type WEBTEC LT300-FM-B-B-6 with the measured flow rate range of 8-300 lpm at the maximum pressure of 420 bar. It outputs a digital frequency signal in the range between $20 \mathrm{~Hz}$ and $2000 \mathrm{~Hz}$ (from $30 \mathrm{mv}$ to $10 \mathrm{~V}$ sinusoidal wave output signal) and has the inductance of $1.55 \mathrm{H}$. By using a zero-detection mechanism; it is possible to compute the frequency of the output signal. It is measured by the use of the NI-9401 module installed on the cRIO-9068 chassis. The module has a special mode (STL) that can automatically track a period of a signal, hence used as a counter in the acquisition unit.

Initial tests of the flow meter showed that due to its high inductance, it outputs a high frequency signal (up to $700 \mathrm{lpm}$ ) that stabilises at a flow rate $>351 \mathrm{pm}$. It makes it impossible to use the device to measure the flow rates $<351 \mathrm{pm}$, hence test the valve at low voltage step tests $(<5 \mathrm{~V})$. This behaviour is shown in Fig. 4.14(a).

For real experimental testing, the flowmeter was placed into the hydraulic circuit (Fig. 4.14(b)). Its measurements were employed for the parameterisation of the proportional valves and hydraulic motor. The flowmeter has two outputs "Frequency + and -". The negative output is connected to the $0 \mathrm{~V}$ input of the Schmidt trigger. Whereas the positive output is connected to the Signal input of the trigger. Pin 17 of the 9401 module is wired in with the common (0V) output. Meanwhile Pin 16 is connected to the output A of the Schmidt circuit.

In order to power up the trigger, another power supply is used to gen- 
erate $+10 \mathrm{~V}$ and $-10 \mathrm{~V}$. The common wire from the power supply is connected to the common terminal of the circuit.

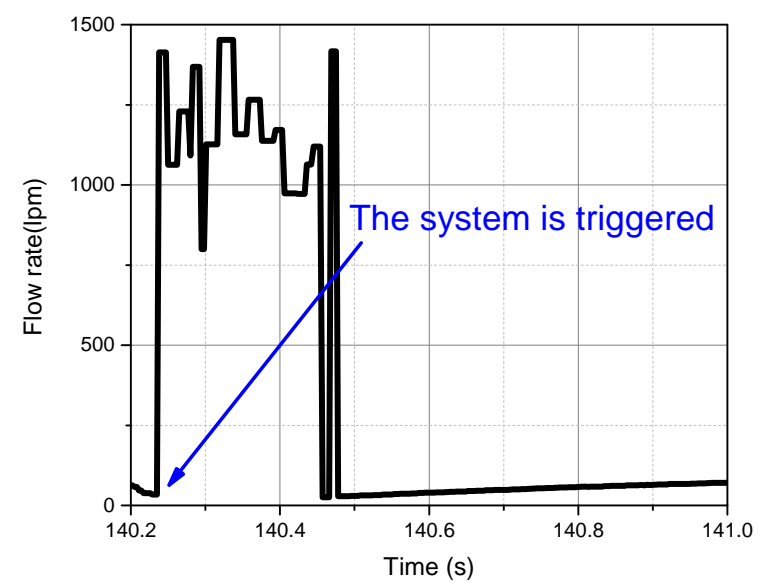

(a) Flow rate spikes during a start of the prototype.

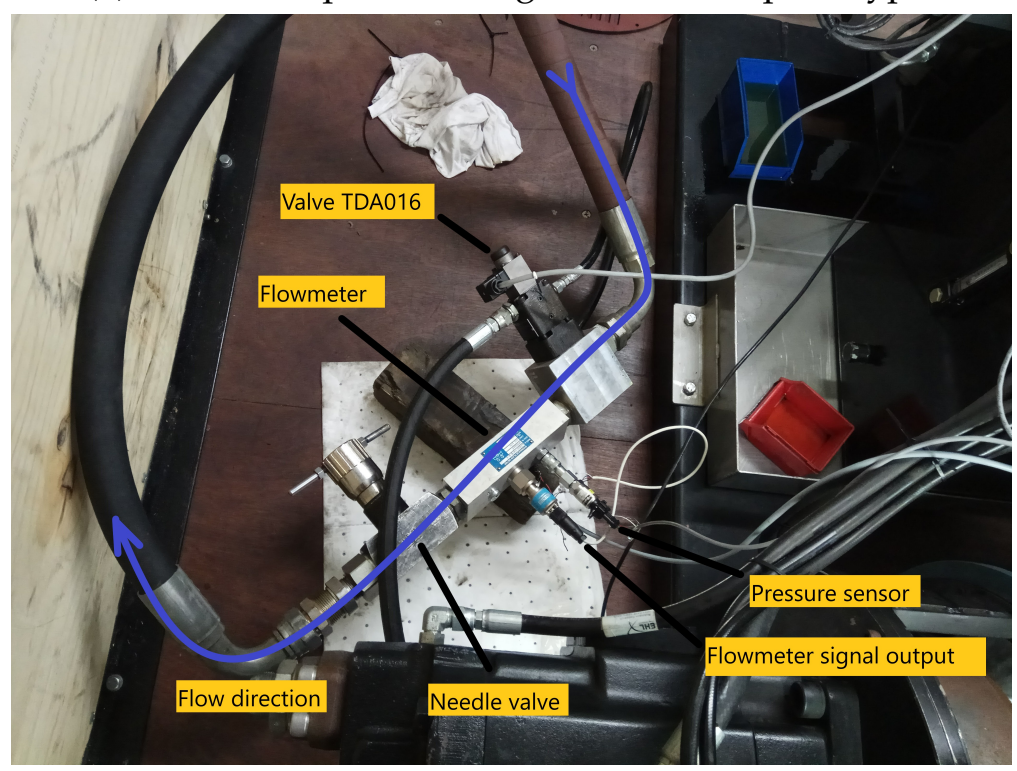

(b) Flowmeter in the hydraulic circuit during experimental testing.

Figure 4.14: Flowmeter testing.

The Schmitt trigger utilised in the project has the following characteristics:

- Input sine waveform from $10 \mathrm{~Hz}$ through to $2000 \mathrm{~Hz}$. Input level as designed and tested is a minimum of $30 \mathrm{mV}$ peak $=21 \mathrm{mV}$ RMS.

- Outputs available from three output terminals = A, B, C.

- Output 'A' provides standardised pulse width of about 200 microseconds (subject to comment below) independent of frequency or input level. 
- The pulses are negative going 5volt.

- Output 'B' provides pulses of varying width depending on frequency and are also negative ging 5volt.

- Output 'C' provides the raw output from the LM311 comparator.

Originally designed with a CA3140 op-amp given a gain of 380 that drove a comparator made with $\mathrm{CD} 74 \mathrm{HC} 14$. The schmitt trigger inverters also provided a standardised pulse width output, together with separate inverted and noninverted outputs. The LM311 produces a pulse for both positive and negative halves of the sine input waveform. The experimental testing of the trigger circuit and the flowmeter is shown in Fig. 4.15 and the circuit is shown in Fig. 4.16 . 


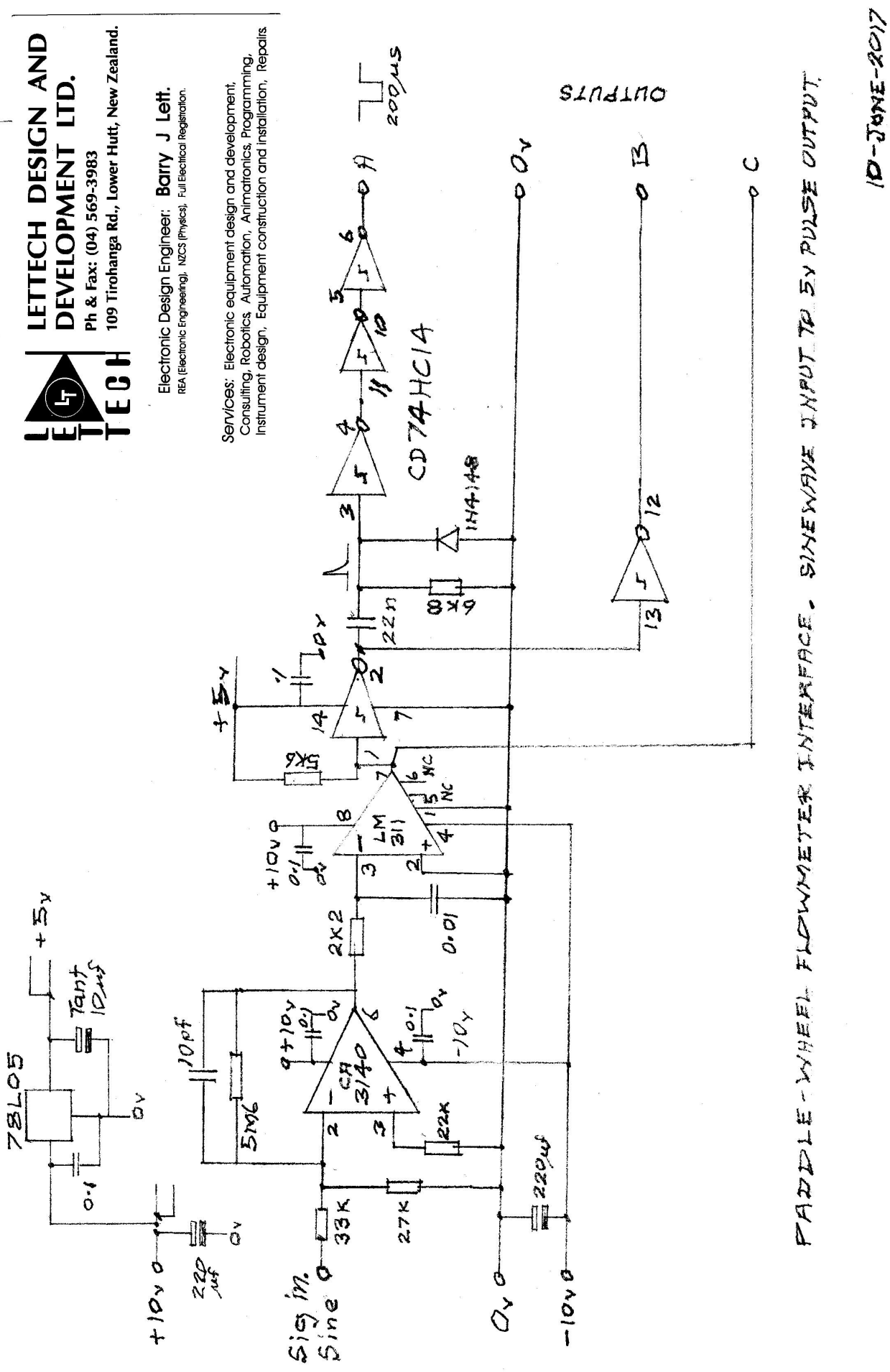

Figure 4.15: Schmitt triggerr circuit 

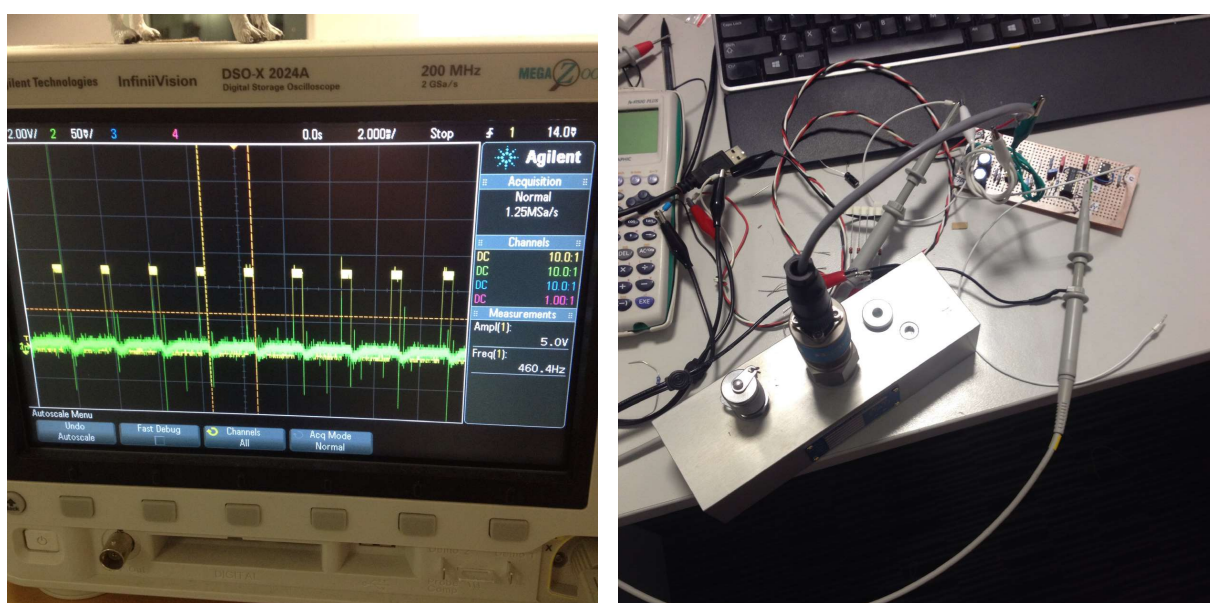

Figure 4.16: Flowmeter testing. Left: Zero detecting mechanism is shown on the oscilloscope. Right: Connected flowmeter and the schmitt trigger circuit.

- Fork sensors The fork sensors installed on the pistons of the system are WFM80-60P311 and WFM80-60P321. Their output signals control the pneumatic valves that control the operation of the pneumatic cylinders. The sensors are depicted in Fig. 4.17

The cylinders are designed to create a constant flow in the hydraulic circuit, acting like a two-stroke engine.

\subsubsection{Mechanical limitations of the designed drivetrain}

Since the generator accelerates due to the high transmitted torque from the hydraulic motor to the generator's shaft, it is required to verify that the torque will not exceed the shear stress of the stepped circular rotor. Firstly it is required to estimate this value for the coupling between the generator and the motor. Secondly, this calculation should be done for the smallest diameter in the generator's shaft [111, 112].

For the coupling the following values have been used:

- The diameter of a larger shaft section D - $80 \mathrm{~mm}$

- The diameter of a smaller shaft section $\mathrm{d}-50 \mathrm{~mm}$

- Radius of the couple bevel r - $30 \mathrm{~mm}$

- Transmitted torque T - $647 \mathrm{~N}^{*} \mathrm{~m}$

- Design factor - 2. This value is the ratio of failure stress to maximum allowable stress. It is usually defined by a specific application and policy (standard). 


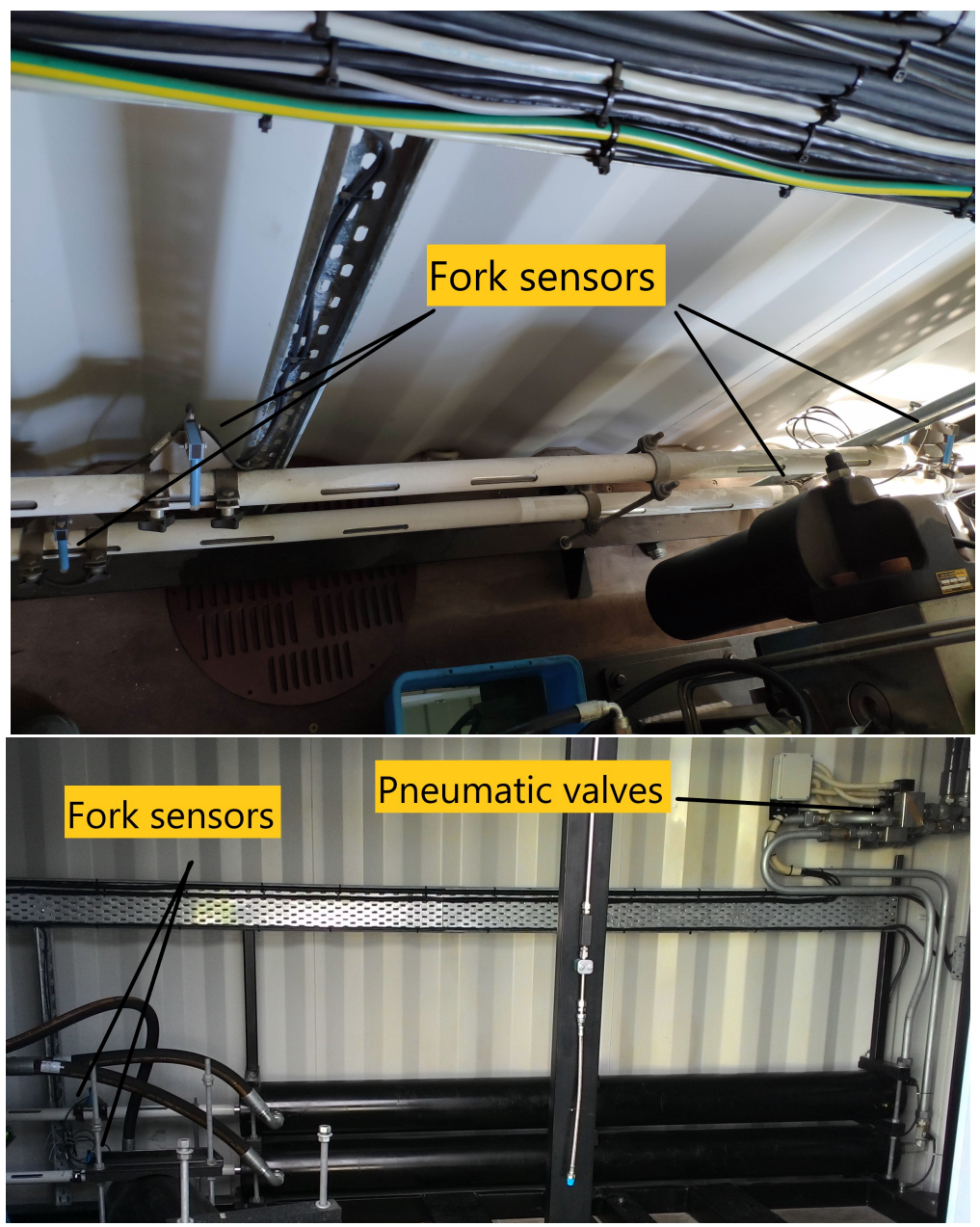

Figure 4.17: Fork sensors installed on the rods of the pneumatic pistons.

The following equations should be used

$$
\begin{gathered}
C_{1}=0.905+0.783 \sqrt{h / r}-0.075 h / r \\
C_{2}=-0.437-1.969 \sqrt{h / r}+0.5533 h / r \\
C_{3}=1.557+1.073 \sqrt{h / r}-0.578 h / r \\
C_{4}=-1.061+0.171 \sqrt{h / r}+0.086 h / r \\
K_{t}=C_{1}+C_{2}(2 h / D)+C_{3}(2 h / D)^{2}+C_{4}(2 h / D)^{2}=1.08 \\
\tau_{\text {nom }}=\frac{16 T}{\pi \cdot d^{3}}=26.36 M P a \\
\tau_{\text {max }}=K_{t} \cdot \tau_{\text {nom }}=28.38 M P a
\end{gathered}
$$


where $h$ is the depth of the groove in the rotor's shaft $(\mathrm{mm}), r$ is the radius of the groove $(\mathrm{mm}), D$ is the notch diameter $(\mathrm{mm}), \tau_{\text {nom }}$ and $\tau_{\max }$ are the nominal and maximum twisting stress respectively ( $\mathrm{MPa}), T$ is the applied torque $(\mathrm{N} \cdot \mathrm{m}), \mathrm{d}$ is the smaller diameter of the shaft $(\mathrm{mm}), K_{t}$ is the elastic torsional load.

After that, the principal stress and maximum shear stress should be calculated for plane stress case. The shear stress, in this case, will be $\tau_{x y}$ $=28.38 \mathrm{MPa}$. According to the maximum shear stress, this design and these conditions are acceptable for the shaft. The next step in the design of the system is the derivation of the maximum transmitted torque through the shaft. Since the maximum shear stress of rolled steel (the generator's shaft material) is $179 \mathrm{MPA}$ and based on the design factor of 2 , the maximum and minimum principal shear stress are $\pm 44.75 \mathrm{MPA}$ :

$$
\begin{gathered}
\tau_{\text {nom }}=\frac{\tau_{\max }}{1.08}=41.435 \mathrm{MPA} \\
T=\frac{\pi \cdot \tau_{\text {nom }} \cdot d^{3}}{16}=\frac{\pi \cdot 41.435 \cdot 10^{6} \cdot 0.05^{3}}{16}=1016 \mathrm{~N} \cdot \mathrm{m}
\end{gathered}
$$

This value of $\mathrm{T}$ indicates the maximum torque that can be transmitted to the shaft under the specified design factor conditions [113-115]. It is needed to verify if the hydraulic motor is able to achieve this torque thus imposing limitations on the system. Based on the nominal values of the hydraulic motor:

- Maximum displacement is $229.5 \mathrm{cc} / \mathrm{rev}$

- Maximum velocity is $2400 \mathrm{rpm}$

- Maximum differential pressure of 420bar

- Maximum torque of $1250 \mathrm{Nm}$

The maximum torque ( $T_{\max }$ that can be achieved in the studied prototype can be calculated taking into account the $\Delta P=350$ (pre-charged accumulator pressure) bar maximum:

$$
T_{\max }=\frac{\nu \cdot \Delta P \cdot Q}{N_{R P M}}=1068 N \cdot m
$$

where $\mathrm{Q}$ is the flow rate through the motor in lpm, $N_{R P M}$ is the speed of the motor in RPM, $\nu$ is the volumetric efficiency.

In the case of the design factor of 2, this torque fails to satisfy the maximum shear stress criteria (also known as the Tresca condition). Hence in the case of the full displacement of a motor of $229.5 \mathrm{cc} / \mathrm{rev}$, the motor 


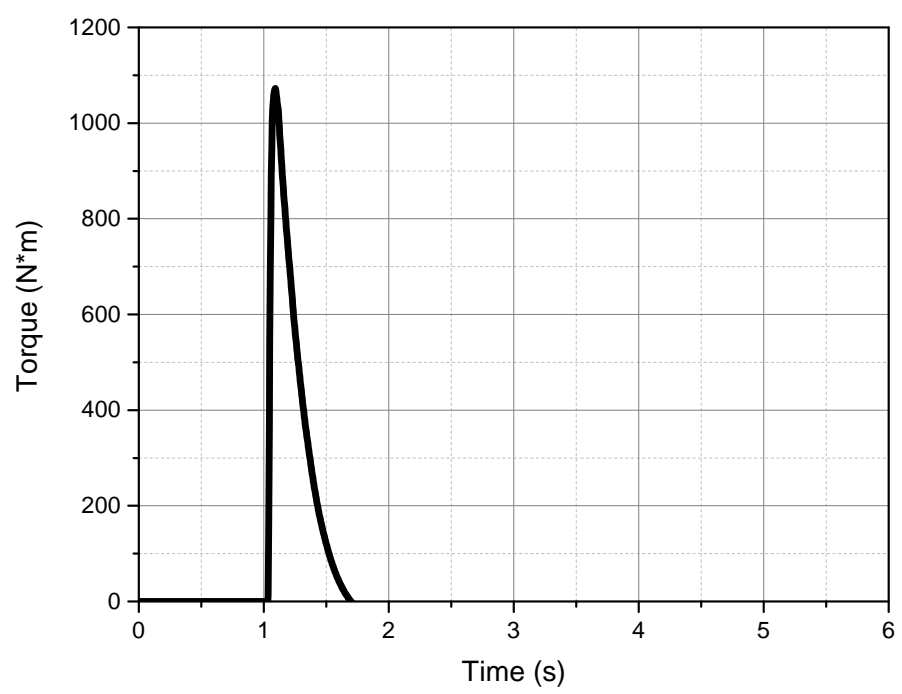

Figure 4.18: Simulated start up torque at the maximum control valve opening.

can achieve the torque that will be large enough to fail the MSS criteria. This displacement can be achieved by changing the inclination of the swash plate by using the swash plate drive. Hence a safety system (or a pressure limiter) should be employed in this isolated circuit to verify that the displacement will be less than the maximum value at the start of the system (when the pressure is 350 bar). The mathematical model also confirms the presented calculations - Fig. 4.18

The presented in this section calculations done by the $\mathrm{PhD}$ author have been confirmed in ANSYS software by running mechanical stress analysis, and the plots are presented in Fig. 4.19. 

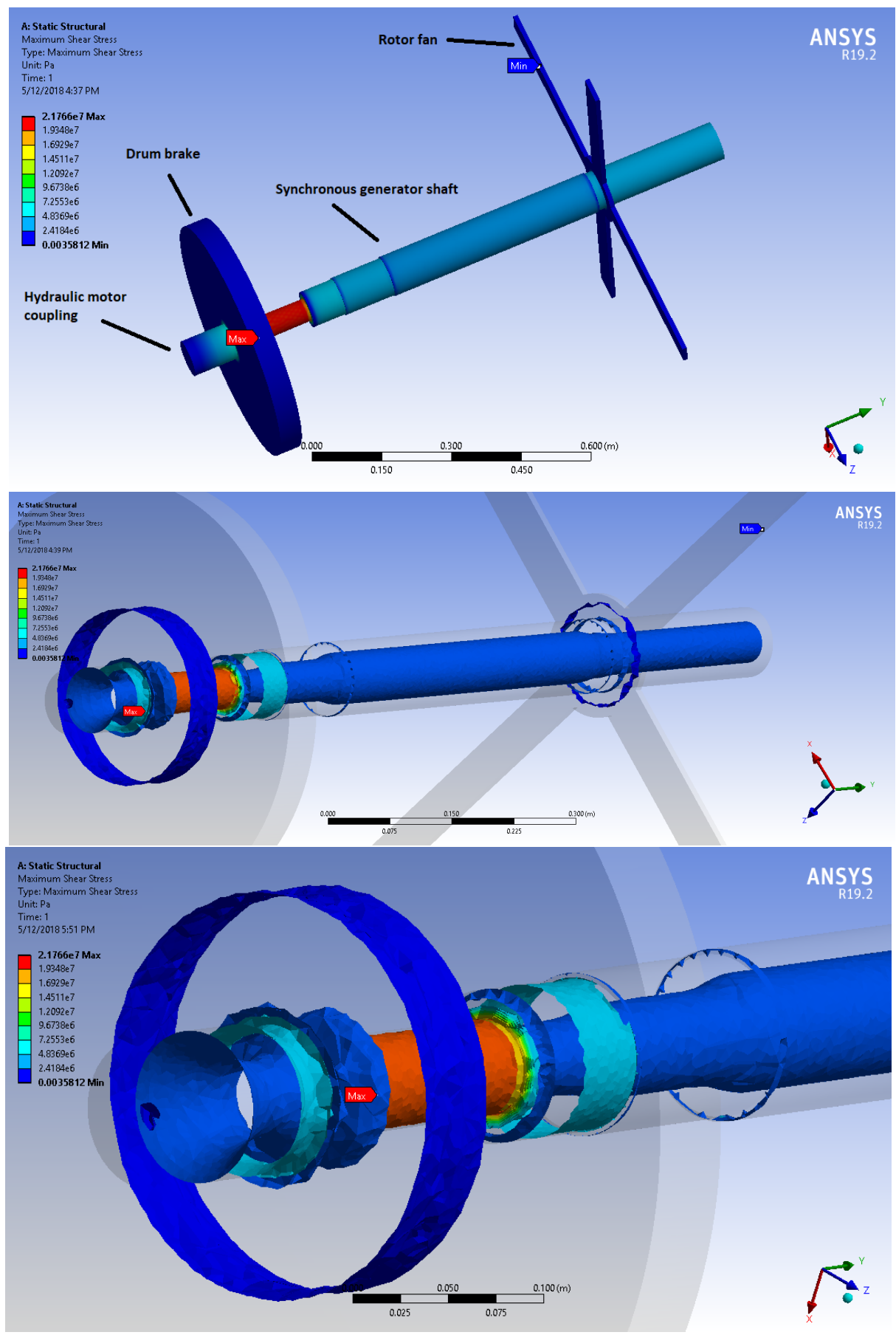

Figure 4.19: Mechanical stress analysis of the generator shaft. 


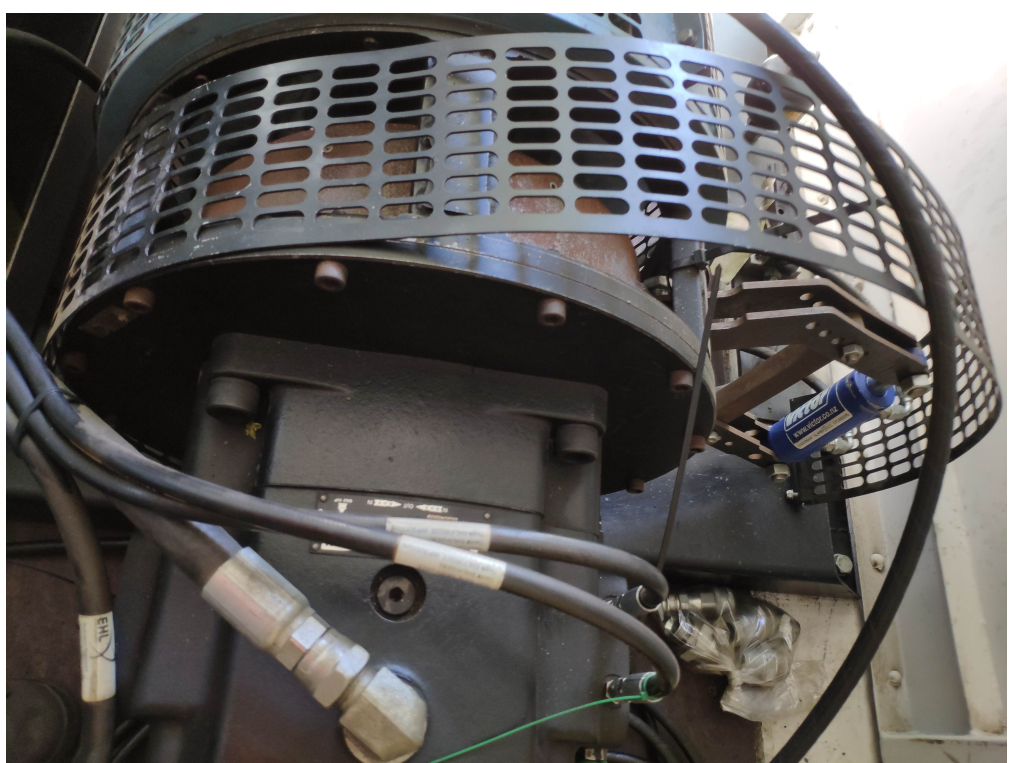

Figure 4.20: Hydraulic drum brake installed on the coupling between the hydraulic motor and the synchronous generator.

It can be seen from Fig. 4.18 and Fig. 4.19 that the derived shear stress is similar to the value calculated in ANSYS. The stress areas also show that the highest stress is experienced around the smallest diameter of the generator shaft.

\subsubsection{Design of the hydraulic drum brakes}

The overall safety of the system is achieved through the use of multiple control loops and also pneumatic and hydraulic safety components. However, in case of emergency when other systems fail to perform, it is feasible to have one safety system that is controlled via a separate controller. This device is a hydraulic drum brake that is placed on the shaft between the hydraulic motor and the generator (shown in Fig. 4.20. In the current prototype, the hydraulic brake was designed and assembled by the client - EHL Group (New Plymouth, Taranaki). However other calculations were performed by the PhD author using [69] to ensure that it is possible to use this type of brake to emergency stop the hydraulic drivetrain..

Since the space around the shaft's coupling is limited, the decision was made to use hydraulic, not pneumatic drum brakes (due to the higher power density). As was calculated earlier in the project, the maximum torque that can be achieved by the drivetrain is $1068 N \cdot m$. By using a 
safety factor of 2 , the braking torque $\left(T_{\text {brake }}\right)$ should be:

$$
T_{\text {brake }}=T_{\text {max }} \cdot 2=1068 \cdot 2=2134 N \cdot m
$$

Since the outer diameter of the coupling $D=0.3 \mathrm{~m}$, the brake force $F_{\max }$ $(N)$ can be derived as (with the roundup torque of $2200 \mathrm{~N} \cdot \mathrm{m}$ ):

$$
F_{\text {max }}=\frac{T_{\text {brake }}}{D}=6300 \mathrm{~N}
$$

The next step required is the calculation of the hydraulic piston dimensions that will be able to achieve the required force. Due to the compact space, it is required to have a small piston with a limited rod's travel distance. In order to follow the compliance standards ISO 3320, ISO 3321, and ISO 4393, the following rod and piston were chosen:

- Piston/Bore diameter $R=32 \mathrm{~mm}$;

- Rod diameter $r=16 \mathrm{~mm}$;

- Stroke of the $\operatorname{rod} L=100 \mathrm{~mm}$;

- Oil flow according to the brake's pressure source is $50 \mathrm{lpm}(Q)$

The bore and rod side areas can be calculated as:

$$
\begin{gathered}
A_{\text {bore }}=\pi \cdot R^{2}=804 \mathrm{~mm}^{2} \\
A_{\text {rodside }}=\pi \cdot r^{2}=603.18 \mathrm{~mm}^{2}
\end{gathered}
$$

The volumes on the piston/bore side can be found as:

$$
\begin{aligned}
& V_{\text {bore }}=\pi \cdot R^{2} \cdot L=0.0804 l \\
& V_{\text {rodside }}=\pi \cdot r^{2} \cdot L=0.06 l
\end{aligned}
$$

Since the required force is $6.6 \mathrm{kN} \cdot \mathrm{m}$, the required pressure to drive the rod is:

$$
P=\frac{F_{\max }}{A_{\text {bore }}}=109.424 b a r
$$

Time of the full stroke is:

$$
T=\frac{V_{\text {rodside }}}{Q}=0.072 \mathrm{~s}
$$

This is the time that can be considered as the time of the brakes clamping on the coupling. Since this is an emergency response system, the testing of it was not performed due to the lack of an auxillary pressure source to activate the brakes. It should be noted however, that it is possible to purchase and install commercially available hydraulic drum brakes (truck brakes and so on). 


\subsubsection{Heat sink thermal analysis}

During the first experimental testing, it was noticed that the amplifier was experiencing significant overheating making its operation unstable [116]. To extend the lifespan of the device and not jeopardise the system's operation, the decision was made to improve its cooling system by selecting new heat sinks.

When it comes to the design of electronic systems, it is vital to maintain their operating temperature below $125^{\circ} \mathrm{C}$. This value is usually used as the worst-case junction temperature (maximum of $150^{\circ} \mathrm{C}-25^{\circ} \mathrm{C}$ safety margin). It is important the components' temperature stay as low as possible (within reasonable boundaries) since the failure rate for semiconductor components doubles for each $10^{\circ} \mathrm{C}$ temperature increase above $55^{\circ} \mathrm{C}$.

There are mainly three ways of designing a heat sink cooling system; a system that can be cooled down by natural convection, by a fan, and by liquid cooling. Taking into account the current design of the amplifier, the decision was made to use one of the first two methods:

$$
R_{\theta j a}=\left(T_{j, \max }-T_{a, \max }\right) / P_{\text {Loss }}
$$

A practical approach for calculating the required heat sink size is presented in the official manual for the utilised op-amp OPA548 from Texas Instruments. The junction temperature of semiconductor chips can be derived according to:

$$
T_{J}=T_{A}+P_{D} \cdot R_{\theta J C} \cdot R_{\theta C H}+R_{\theta H A}
$$

where

1. $T_{J}$ - junction temperature $\left({ }^{\circ} \mathrm{C}\right)$, with a $25^{\circ} \mathrm{C}$ safety margin this temperature is $125^{\circ} \mathrm{C}$.

2. $T_{A}$ - ambient temperature $\left({ }^{\circ} \mathrm{C}\right)$. This temperature is assumed to be $30^{\circ} \mathrm{C}$.

3. $P_{D}$ - the dissipated power of the chip $(\mathrm{W})$. The maximum output current is $1.05 \mathrm{~A}$ at $10 \mathrm{~V}$, resulting in $10.5 \mathrm{~W}$ dissipated power.

4. $R_{\theta J C}$ - junction-to-case thermal resistance $\left({ }^{\circ} \mathrm{C} / \mathrm{W}\right)-2.5^{\circ} \mathrm{C} / \mathrm{W}(\mathrm{DC})$ according to the heat sink manufacturer.

5. $R_{\theta C H}$ - case-to-heat sink thermal resistance $\left({ }^{\circ} \mathrm{C} / \mathrm{W}\right)-1^{\circ} \mathrm{C} / \mathrm{W}$ for a $\mathrm{TO}-220$ mounted package.

6. $R_{\theta H A}$ - heat sink-to-ambient thermal resistance $\left({ }^{\circ} \mathrm{C} / \mathrm{W}\right)$

7. $R_{\theta J A}$ - junction-to-air thermal resistance $\left({ }^{\circ} \mathrm{C} / \mathrm{W}\right)$ 

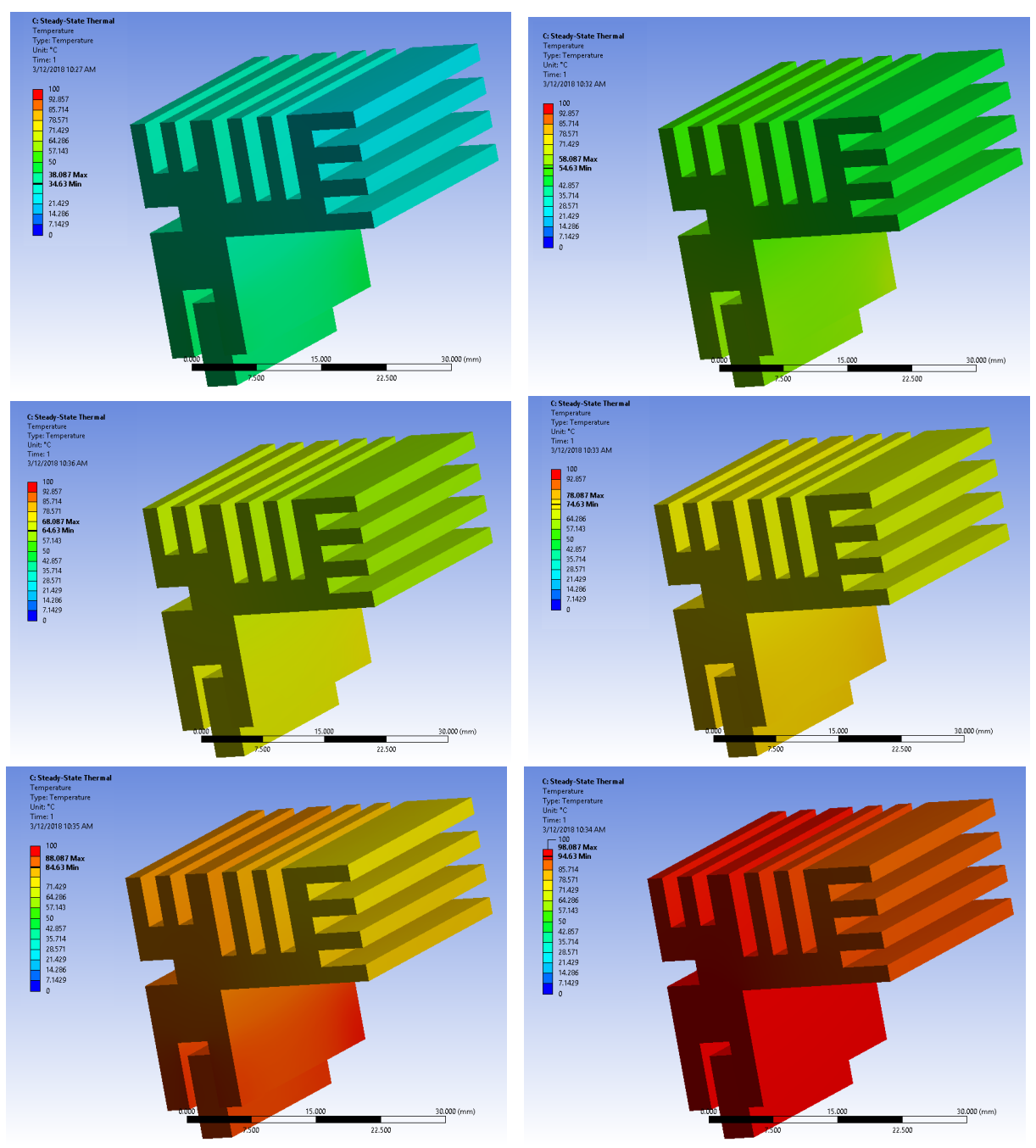

Figure 4.21: Temperature distribution of the aluminum heatsink in the amplifier at different ambient temperatures..

This can be solved for $R_{\theta H A}$ :

$$
R_{\theta H A}=\frac{125^{\circ} \mathrm{C}-30^{\circ} \mathrm{C}}{10.5 \mathrm{~W}}-\left(2.5^{\circ} \mathrm{C} / \mathrm{W}+1^{\circ} \mathrm{C} / \mathrm{W}\right)=5^{\circ} \mathrm{C} / \mathrm{W}
$$

It means that to keep the chip temperature below $125^{\circ} \mathrm{C}$ it is required to choose a heat sink with $R_{\theta H A}$ less than $5^{\circ} \mathrm{C} / \mathrm{W}$. Due to the limited space on and above the circuit board, an SK $12550 \mathrm{~mm}$ was chosen for the chip $\left(R_{\theta H A}=8^{\circ} \mathrm{C} / \mathrm{W}\right)$. To achieve the required cooling effect the decision was made to install a $12 \mathrm{Vdc}$ fan into the amplifier enclosure. According to the official heat sink manuals from Crydom show that a $40 \mathrm{~mm}$ fan introduces a correction factor of 0.439 , meaning that the effective heat sink thermal resistance is $8^{*} 0.439=3.5^{\circ} \mathrm{C} / \mathrm{W}$. This value satisfies the previously derived thermal resistance value. By running experiments, the operating temperature of the chip was confirmed to be below $100^{\circ} \mathrm{C}$ under different operation cycles.

To verify the previously presented calculations, it is possible to model the 
heatsinks at different ambient temperatures. For these purposes, the ANSYS software was utilised, and the results plots are depicted in Fig. 4.21

It can be seen that at the ambient temperature of $30^{\circ} \mathrm{C}$ the chip temperature reaches almost $100^{\circ} \mathrm{C}$ which can be considered the maximum safe operating temperature.

To avoid overheating of the components, a fan was installed into the amplifier enclosure. By creating constant flow in the enclosure and forcing heat dissipation, the temperature in the enclosure has never exceeded $18-20^{\circ} \mathrm{C}$. The solution proved to be reliable over all stages of the prototype's experimental testing. The final results of these alterations are presented in Fig. 4.22.

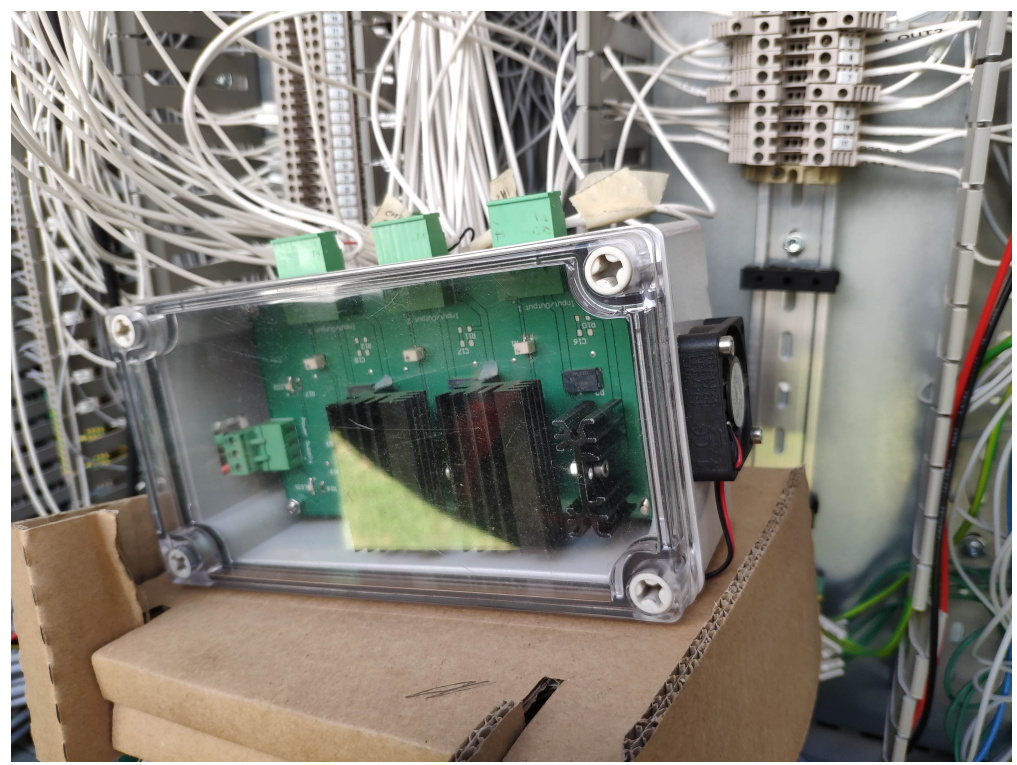

Figure 4.22: Final design of the amplifier for the ss-CAES prototype.

\subsection{Chapter Summary}

In this chapter, a description of the ss-CAES prototype and its main components was given. The remainder of the chapter discussed some of the mechanical modifications that had been done on the prototype. The implemented by the PhD author high-speed data acquisition system was presented in the chapter as well. 


\section{Chapter 5}

\section{Hydraulic valve characterisation}

The electro-hydraulic drivetrain is controlled by the employed proportional throttle valve (TDA016). This section elaborates on Step 2 in the prototype's validation process (see Fig. 5.1), focusing on the valve parameterisation. It took place in September 2017 in New Plymouth, Taranaki, New Zealand.

Traditionally a control valve (proportional throttle valve) is modelled with the use of one transfer function and a 2D look-up table. The first one describes the dynamics of the modelled control valve (control stage) and the second one focuses on the pressure-flow rate characteristics (power stage). The experimental results were used in the corresponding mathematical model to increase its accuracy and reliability. Firstly, the relevant methods and mathematical models for such parameterisation are described in the chapter. Secondly, the data acquisition system for these measurements is presented. Finally, the experimental results are shown in comparison to their mathematical simulation.

An overview of the components and sensors employed for this testing is presented in Fig. 5.2. The hydraulic motor was bypassed to connect the downstream hose from the proportional valve back to the HPU.

\subsection{Characterisation of the valve control stage}

The control stage of the valve depicts the correlation between the opening dynamics of the valve over time (measured flow rate as the function of step tests). There are a few limitations for the experimental testing that should be noted:

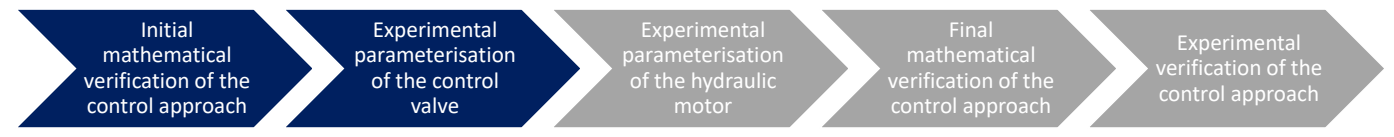

Figure 5.1: Experimental validation of the synchronisation approach. Current stage is the control valve parameterisation (step 2). 


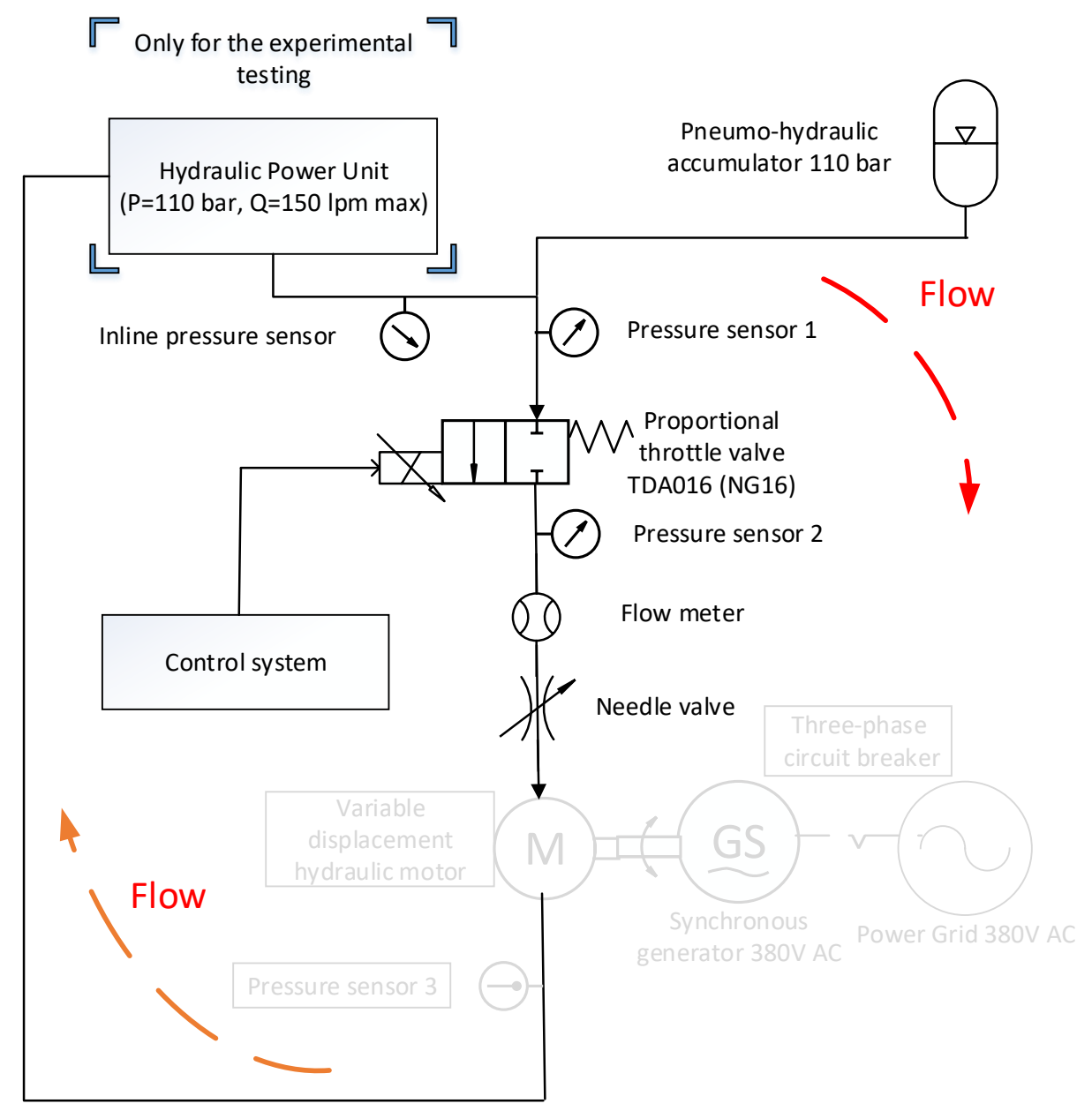

Figure 5.2: Schematic of the data acquisition system utilised for the experimental testing of the control valve NG16.

- The maximum flow rate is $150 \mathrm{lpm}$, which is the maximum flow output from the hydraulic power unit.

- The maximum inline pressure is $110 \mathrm{bar}$.

- The minimum measured flow rate is $>35-40 \mathrm{lpm}$ due to the limitation of the utilised flow meter.

Taking these points into account, the opening range for the testing lies between 40 and $80 \%$ (depending on the differential pressure). Remembering that the higher the differential pressure across the valve, the higher the flow rate through the orifice opening area, since [81].

$$
Q=C_{v} \sqrt{\frac{\Delta P}{S G}}
$$

where $\mathrm{Q}$ is the flow rate through the valve (lpm), $C_{v}$ is the flow coefficient, $\Delta P$ is the differential pressure (bar), $S G$ is the specific gravity. 
To verify the dynamics of the proportional throttle valve, it is required to perform a series of step tests at different openings, in the range of $5.5 \mathrm{~V}-8$ $\mathrm{V}$ with the responses depicted in Fig. 5.3 [78]. A close comparison of these transients depicts the complex behaviour of the valve's poppet since the flow rate decreases after the initial opening when the input voltage ranges from $6 \mathrm{~V}$ to $8 \mathrm{~V}$, due the minimum and maximum flow rate limitations.

It is followed by a flow rate rise to its steady-state value corresponding to the input voltage signal (opening). After a close look at the plot, it can be observed that a first order transfer function describes the low voltage step tests $(5.5 \mathrm{~V} \mathrm{~V})$ [117]:.

$$
W(s)=\frac{a_{0}}{b_{1} \cdot s+b_{0}}
$$

It contradicts the standard mathematical approach stating that the dynamics of a proportional throttle valve could be described as a standard second-order function [118]:

$$
H(s)=\frac{a_{0}}{b_{2} \cdot s^{2}+b_{1} \cdot s+b_{0}}
$$

However, at a voltage higher than $6 \mathrm{~V}$ a transfer function with three poles and two zeros describes the dynamics more closely.

$$
W(s)=\frac{a_{2} \cdot s^{2}+a_{1} \cdot s+a_{0}}{b_{3} \cdot s^{3}+b_{2} \cdot s^{2}+b_{1} \cdot s+b_{0}}
$$

To increase the accuracy of the designed mathematical model, it is required to increase the order of the valve's control stage transfer function and employ 3-pole transfer functions with their coefficients given in Table. 5.1. The step response curves of these transfer functions are depicted in Fig. 5.3 as the simulation curves.

\begin{tabular}{|c|c|c|c|c|c|c|c|}
\hline $\begin{array}{l}\text { Control voltage }(\mathrm{V}) \backslash \\
\text { Transfer function coefficients }\end{array}$ & $a_{2}$ & $a_{1}$ & $a_{0}$ & $b_{3}$ & $b_{2}$ & $b_{1}$ & $b_{0}$ \\
\hline 5.5 & 0 & 19480 & 434300 & 0 & 1 & 607 & 9398 \\
\hline 5.75 & 5700 & 18200 & 2826300 & 1 & 113 & 1110 & 51940 \\
\hline 6 & 9600 & 25800 & 4985600 & 1 & 154 & 1519 & 75256 \\
\hline 6.5 & 19300 & 68400 & 8226600 & 1 & 205 & 2508 & 90427 \\
\hline 7 & 13460 & 99900 & 4702100 & 1 & 121 & 2295 & 41150 \\
\hline 7.5 & 45000 & 416000 & 10845000 & 1 & 359 & 6638 & 81884 \\
\hline 8 & 44600 & 530100 & 9810000 & 1 & 321 & 7254 & 66984 \\
\hline
\end{tabular}

Table 5.1: Parameterisation of the valve's control stage. Coefficients for the second and third order transfer functions 


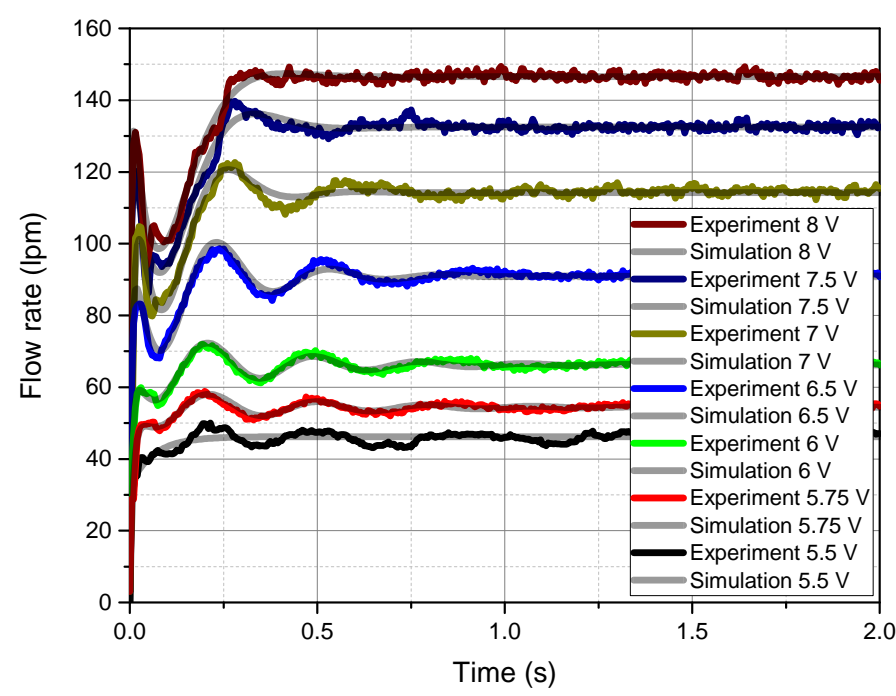

Figure 5.3: Flow rate through the valve TDA016 during the step response tests at different opening voltage (5.5-8 V). Comparison between the experimental curves and the first order $(5.5 \mathrm{~V})$ third-order transfer function fitted curves (5.75-8V).

Table 5.2: Pearson coefficient for the simulated transfer function curves fitted to the experimental step responses

\begin{tabular}{llllllll}
\hline \hline Valve opening (V) & 5.5 & 5.75 & 6 & 6.5 & 7 & 7.5 & 8 \\
\hline Pearson coefficient & 0.88981 & 0.96919 & 0.97938 & 0.97953 & 0.98184 & 0.98729 & 0.99183 \\
\hline
\end{tabular}

From the plotted curves it can be seen that the third order might be the highest possible order for the transfer functions, since there is a risk of overfitting the experimental curves with the fourth order transfer functions. To show the difference in the accuracy of the first and third order transfer functions, it is necessary to use the Pearson coefficients that are given in Table. 5.2 .

Different transfer functions can represent every single step test individually. It is not feasible for mathematical modelling, meaning that it is required to find the average dynamics curve amongst the presented step tests. Some literature sources use only two opening tests at $20 \%$, and $100 \%$ since valve manufacturers usually provide these curves [78]. Bearing in mind the flow meter limitation $(Q>35 \mathrm{lpm})$ and the HPU maximum flow rate $(\mathrm{Q}=150 \mathrm{lpm})$, these tests cannot be performed. Therefore, the best option is to use the whole experimental set of step tests to find one average experimental curve. Then a curve should be fitted to it, and its derived transfer function can be used later in the mathematical model.

As an example of this behaviour and approach, a normalised step test along 


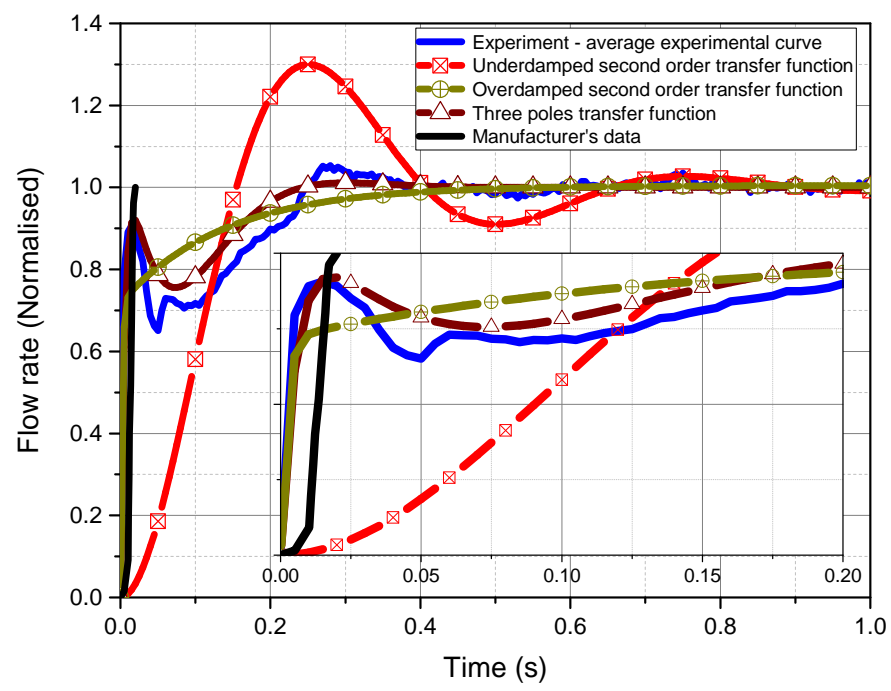

Figure 5.4: Normalised average experimental curve representing the control stage of the TDA016 valve. Fitted curves were used for the modelling of the control stage, with the best fit of the third order transfer function.

Table 5.3: Comparison between the second and third order transfer functions for the fitted normalised average experimental curve

\begin{tabular}{llllllll}
\hline \hline & $\mathrm{a} 2$ & $\mathrm{a} 1$ & $\mathrm{a} 0$ & $\mathrm{~b} 3$ & $\mathrm{~b} 2$ & $\mathrm{~b} 1$ & $\mathrm{~b} 0$ \\
\hline Third order transfer function & $0.0186 \mathrm{e} 4$ & $0.2865 \mathrm{e} 4$ & $6.2996 \mathrm{e} 4$ & $0.0001 \mathrm{e} 4$ & $0.0194 \mathrm{e} 4$ & $0.4958 \mathrm{e} 4$ & $6.2964 \mathrm{e} 4$ \\
Overdamped second order function & 0 & $0.3401 \mathrm{e} 3$ & $3.4258 \mathrm{e} 3$ & 0 & $0.0010 \mathrm{e} 3$ & $0.4779 \mathrm{e} 3$ & $3.4106 \mathrm{e} 3$ \\
Second order transfer function & 0 & 0 & 1 & 0 & $8.9 \mathrm{e}-4$ & 0.02135 & 1 \\
\hline
\end{tabular}

with the corresponding first, second, and third order transfer functions (obtained via Simulink) is presented in Fig. 5.4. It can be seen from the plot that the simulated curve (a second-order transfer function) is drastically different from the experimental results in the first 10-20 ms after the initial opening. Meaning that the transfer function's output is accurate when the valve is in its steady state; however, the initial dynamics of the function do not depict the real behaviour. It introduces an error into the modelling results, especially during rapid start-ups and step responses. The corresponding coefficients of the transfer functions from Fig. 5.4 are summarised in Table. 5.3.

For the modelling of the control stage subsystem, it is necessary to find the most accurately fitted curve amongst three different transfer function responses. It can be illustrated by the use of the Pearson coefficient - Table. 5.4.

The coefficients show that a 3-pole transfer function best fits the normalised experimental average curve and was therefore used in the final version of the mathematical model. 
Table 5.4: Pearson coefficients comparison between the second and third order transfer functions for the fitted normalised average experimental curve

\begin{tabular}{llll}
\hline \hline Transfer function & Underdamped & Overdamped & Three pole \\
\hline Pearson coefficient & 0.68947 & 0.88933 & 0.95419 \\
\hline
\end{tabular}

The next step in the verification of the derived transfer function is the comparison between the experimental step flow rate profiles and the average simulated flow rates, as it is shown in Fig. 5.5. The biggest error between the flow rates can be observed within the first $0.25-0.4 \mathrm{~s}$ from the start. The flow rate discrepancies are summarised in Fig. 5.6. This difference proves the statement that in case of accelerating the electro-hydraulic drivetrain as quickly as possible, this flow rate errors can make a significant difference in the tuning process of the control system. It also means that the behaviour of the drivetrain in the real ss-CAES slightly deviates from the model.

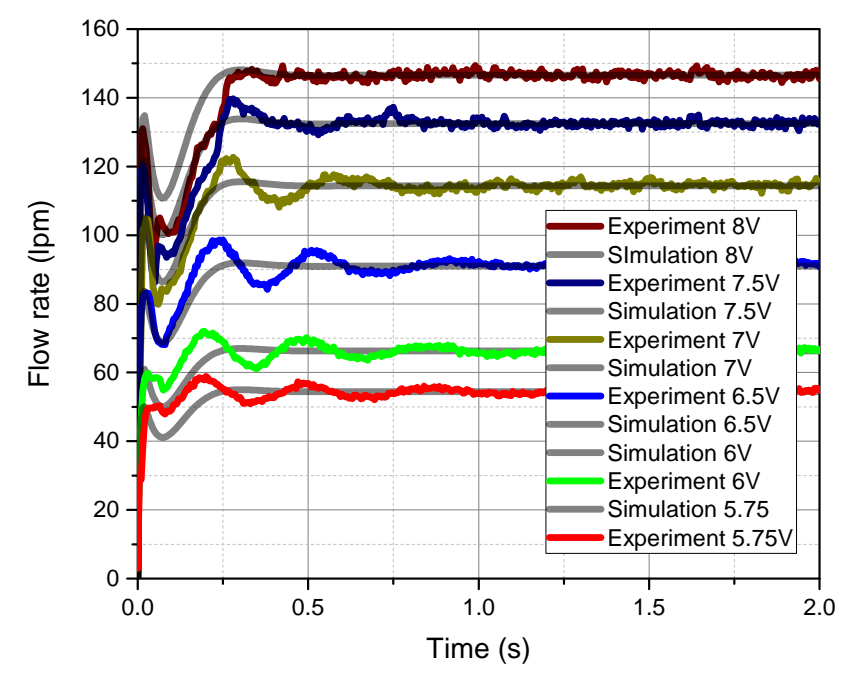

Figure 5.5: Comparison between the experimental step input flow rates and the simulated average transfer function.

This disadvantage can be eliminated by employing multiple transfer function of every experimental step test that has been performed. In Simulink it can be quickly implemented by using a Lookup table with the corresponding transfer functions parameters. However, due to the limited computational power and significant simulation times, this approach was not implemented in the final model.

It should be noted that there are other options of replicating the flow rate profiles apart from the use of transfer functions. By using the same Simulink 


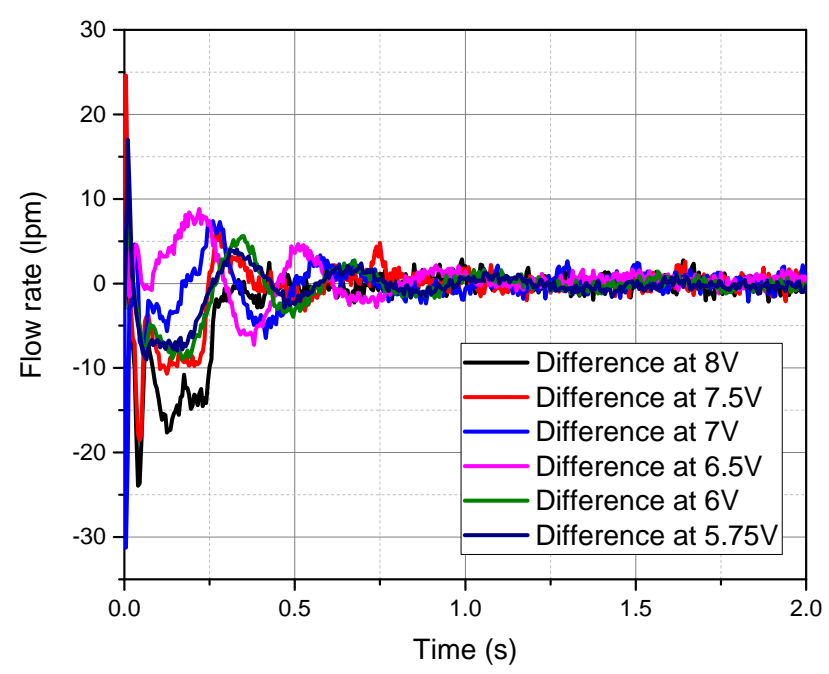

Figure 5.6: Flow rate difference between the experimental flow rates at different step inputs and the simulated average transfer function output.

System Identification toolbox it is possible to estimate any corresponding State Space models. However, these models don't work accurately with the used Simscape models for the electro-hydraulic drivetrain.

Polynomials models were never able to achieve the required fit and could not replicate that initial flow rate sag observed in the first $10-15 \mathrm{~ms}$ from the start. Nonlinear models either had a lower fit value, or were too complex for the model to simulate the behaviour of the drivetrain within a reasonable timeframe.

It should be noted that since the system starts from rest when it is triggered, the valve should be tested from zero voltage/zero flow rate. Non zero tests contradict the logic of the system and also contradicts the approach presented in [78].

\subsection{Characterisation of the valve power stage}

The second part of the modelling of the valve TDA016 is its power stage characterisation. It describes the relationship between the valve's opening (i.e., input control voltage) and the flow rate through it. To design an accurate mathematical model, it is necessary to perform a series of tests at various differential pressure profiles. In case of the presented system, the operating range lies between 10 bar and 100 bar. However, the DAQ system was implemented in the way that the inline pressure from the power unit cannot be changed during the experimental testing. Therefore, to achieve the required differential pres- 


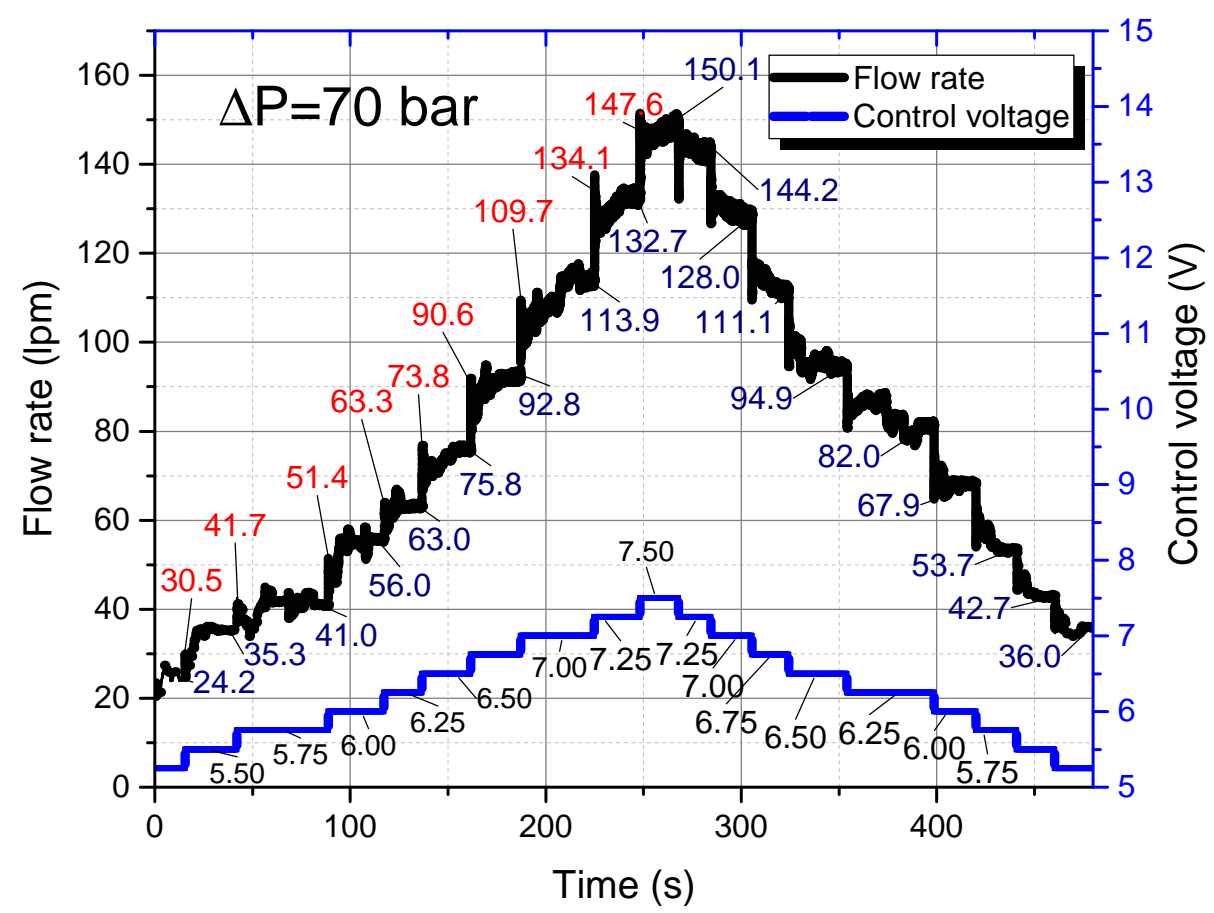

Figure 5.7: Quasi-state flow rate test at different openings (control voltage) at 70 bar differential pressure across the valve.

sure across the valve, a variable orifice area after the valve should be placed. This, in turn, gives the opportunity to change the differential pressure across the valve when it is open.

A needle valve was chosen for this purpose. Since during the testing the differential pressure across the valve changes depending on its opening, the needle valve opening should be altered to achieve the required constant pressure drop across the valve.

A recorded flow rate at this moment is used for the power stage parameterisation of the valve, as it is depicted in Fig. 5.7, for the pressure drop of 70 bar. Since it is necessary to open the valve by increasing the control voltage and then close it by decreasing the voltage, it is possible to record the upward and downward flow rate trends. It helps to verify the valve's operation in the whole operating range under different conditions. As an example of this testing, two flow rate curves are shown in Fig. 5.10. The same tests were repeated for $10 \mathrm{bar} / 20 \mathrm{bar} / 30 \mathrm{bar} / 50 \mathrm{bar} / 70 \mathrm{bar} / 100 \mathrm{bar}$ differential pressure profiles across the valve, and they are presented in Fig. 5.8. The TDA016 differential pressure profiles are depicted in Fig. 5.9. The lower a pressure drop across the valve, the more challenging is to achieve the required needle valve pressure. 

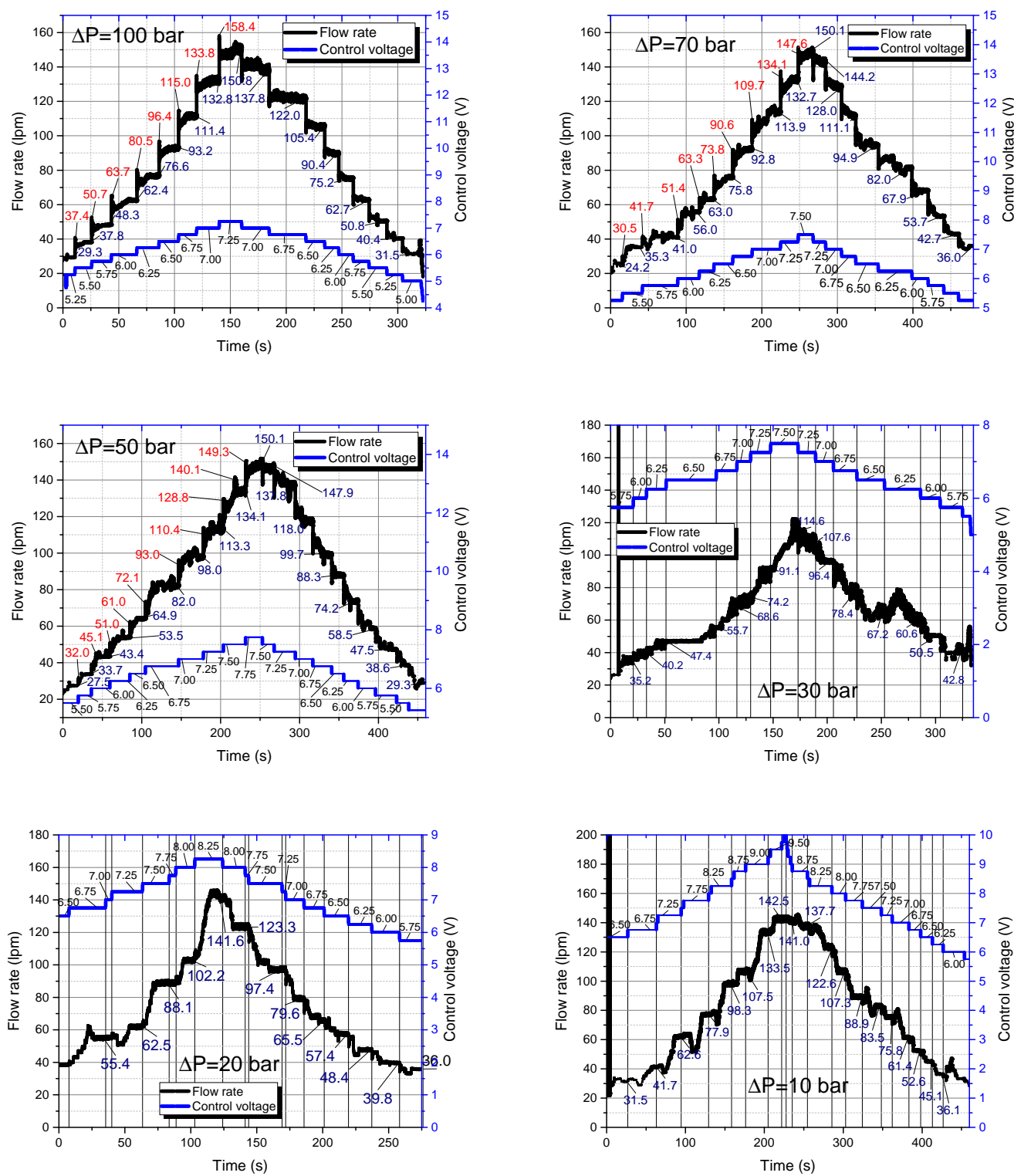

Figure 5.8: Power stage parameterisation - ptv differential pressure.

By summarising all steady-state flow rates at various differential pressure profiles across the valve, it is possible to design a 2D lookup table that later can be used in the final mathematical model of the valve.

\subsubsection{Hysteresis effect in hydraulic valves}

After the close analysis of the previously presented plot (Fig. 5.7), it can be noticed that the flow rates between the opening and closing of the valve are different. This is due to the hysteresis effect in the valve's coil, the magnetisation of which creates the opening force (the valve is spring loaded and normally closed) [76]. The travel distance of the poppet changes due to the residual 

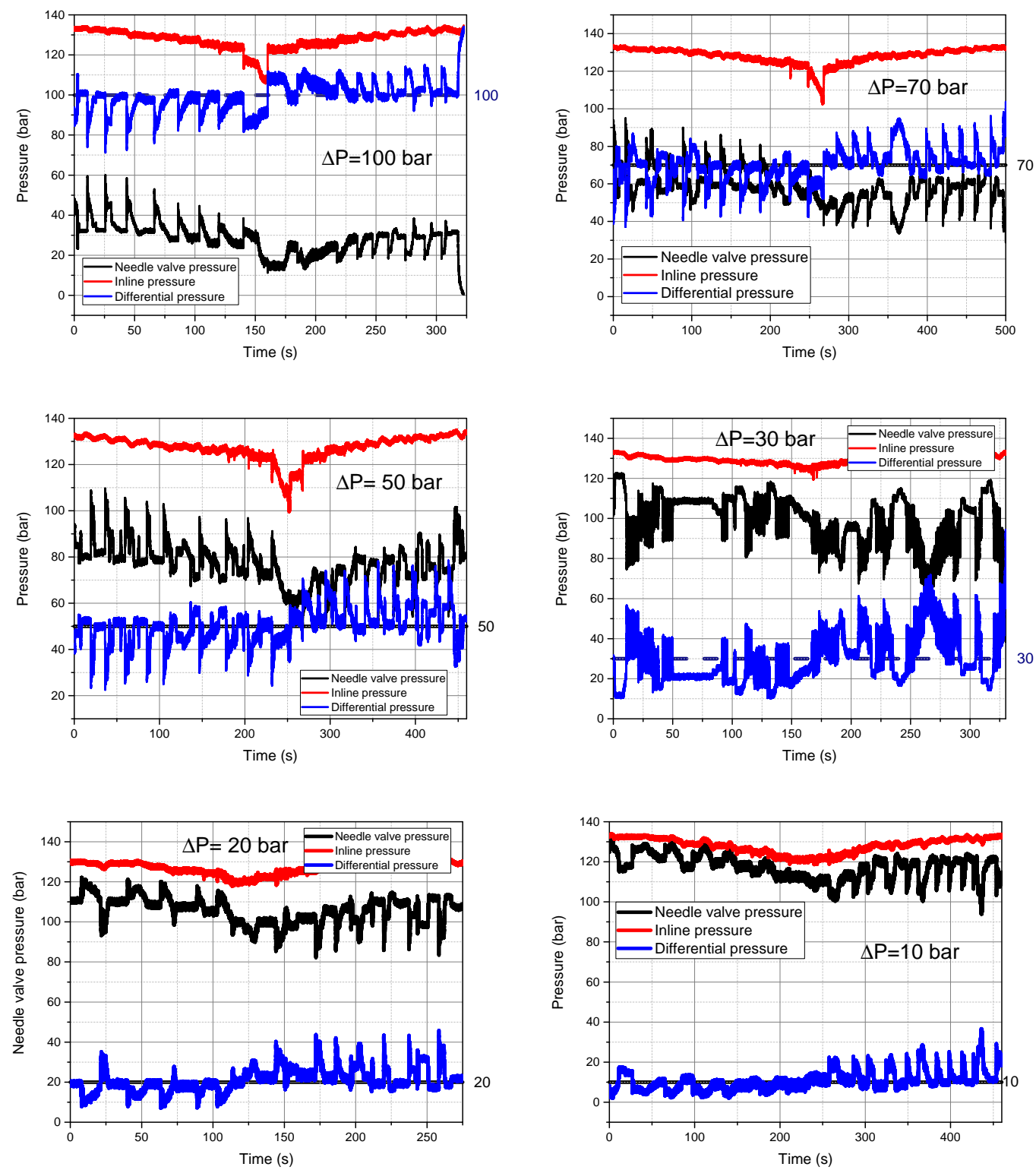

Figure 5.9: Power stage parameterisation - ptv differential pressure.

magnetic field in the coil (hysteresis) causing this flow rate difference, as is shown in Fig. 5.10 .

According to the manufacturer's data, the hysteresis error in this size of the valve (NG16) should be around 3\%, whereas the experimental testing shows the error up to $15 \%$. It becomes even more noticeable when the experimental results are plotted along the official data as it is presented in Fig. 5.11.

However, there is a big difference in the way the aforementioned experimental curves and manufacturer's profiles were acquired. Valve manufacturers (like Parker) test their valves using highly precise and accurate pressure pumps and short tubing connecting them to the tested valves. Whereas, the current experimental setup comprises a standard industrial HPU and a 


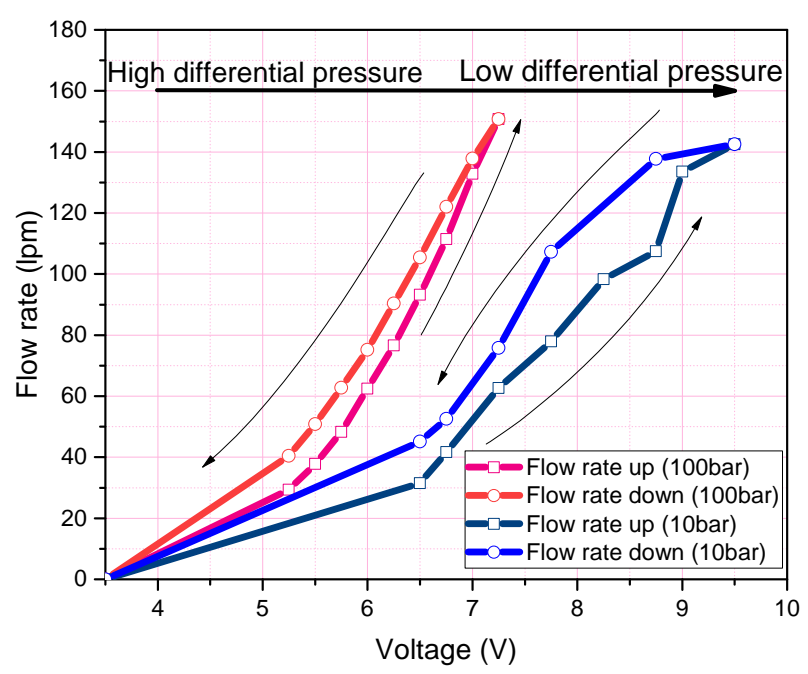

Figure 5.10: Power stage verification of the valve TDA016. The hysteresis effect within the experimental opening range at two differential pressure profiles.

few meters of pipe and hose that are connected to the central manifold. Even though it is different from the "ideal" testing environment, this particular testing helps to verify the realistic behaviour of the valve in the current system.

The next important step in verifying the operation of the valve and the hydraulic drive train is the comparison between the experimental and ideal flow rate curves. Regarding the studied hysteresis effect, the most feasible solution is to compare the flow rate difference between the opening and closing of the hardware and ideal TDA016 valves. At this stage, there is the experimental data obtained for the differential pressure profiles from 10 bar to 100 bar at the opening between $50 \%-80 \%$. To find the flow rates for the opening below $50 \%$ and up to $100 \%$ it is required to extrapolate the gathered experimental measurements. However, it should be done with the use of the valve flow coefficient that can be derived from the experimental data, taking into account that:

$$
C_{V}=Q / \sqrt{\frac{\Delta P}{S G}}
$$

where $C_{V}$ is the flow coefficient, $Q$ is the flow rate in lpm, $\Delta P$ is the differential pressure across the valve (bar), $S G$ is the specific gravity. The derived coefficients are presented in Fig. 5.12 


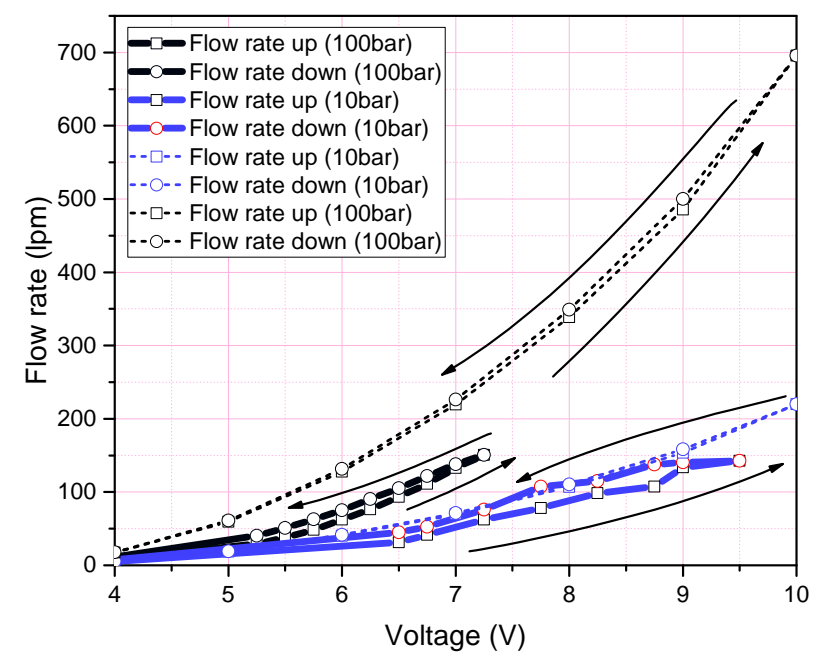

Figure 5.11: Power stage verification of the control valve NG16 with the hysteresis effect.

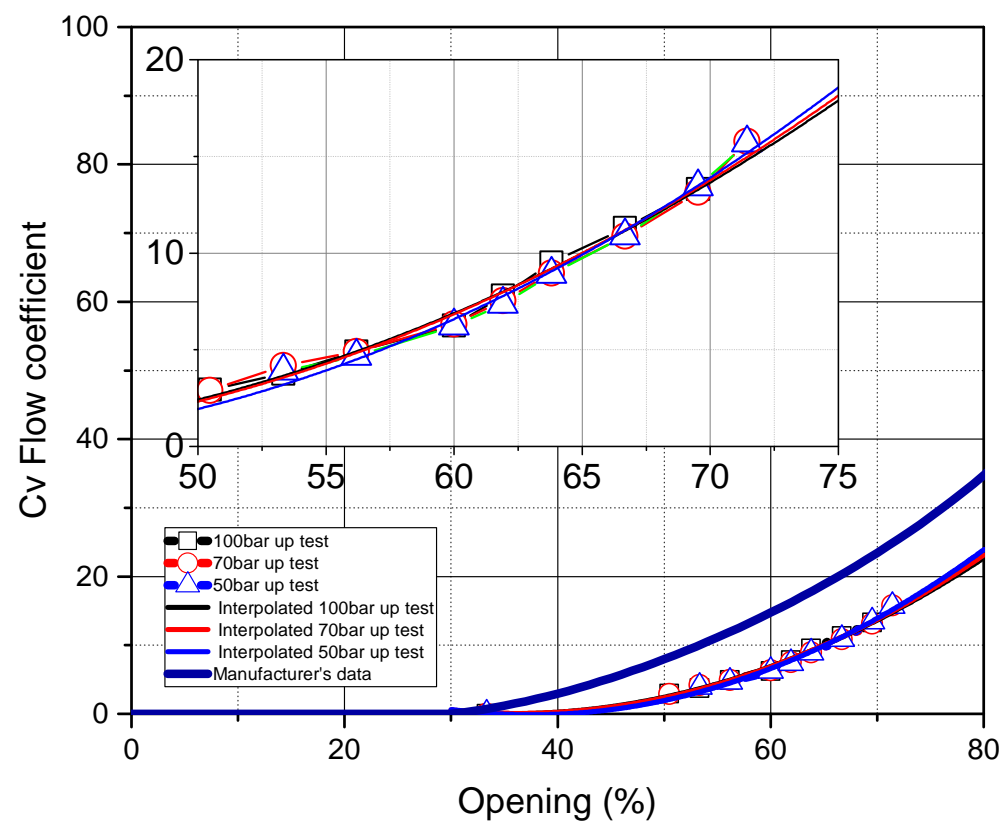

Figure 5.12: Derived flow coefficients of the control valve NG16 from the experimental results during the power stage verification.

After this, it is possible to use this equation and the extrapolated flow discharge coefficients to derive the flow rate profiles in the range of $35-100 \%$ opening of the valve at various differential pressure. The same principle can be used for deriving the ideal valve discharge coefficient, that can be used for 


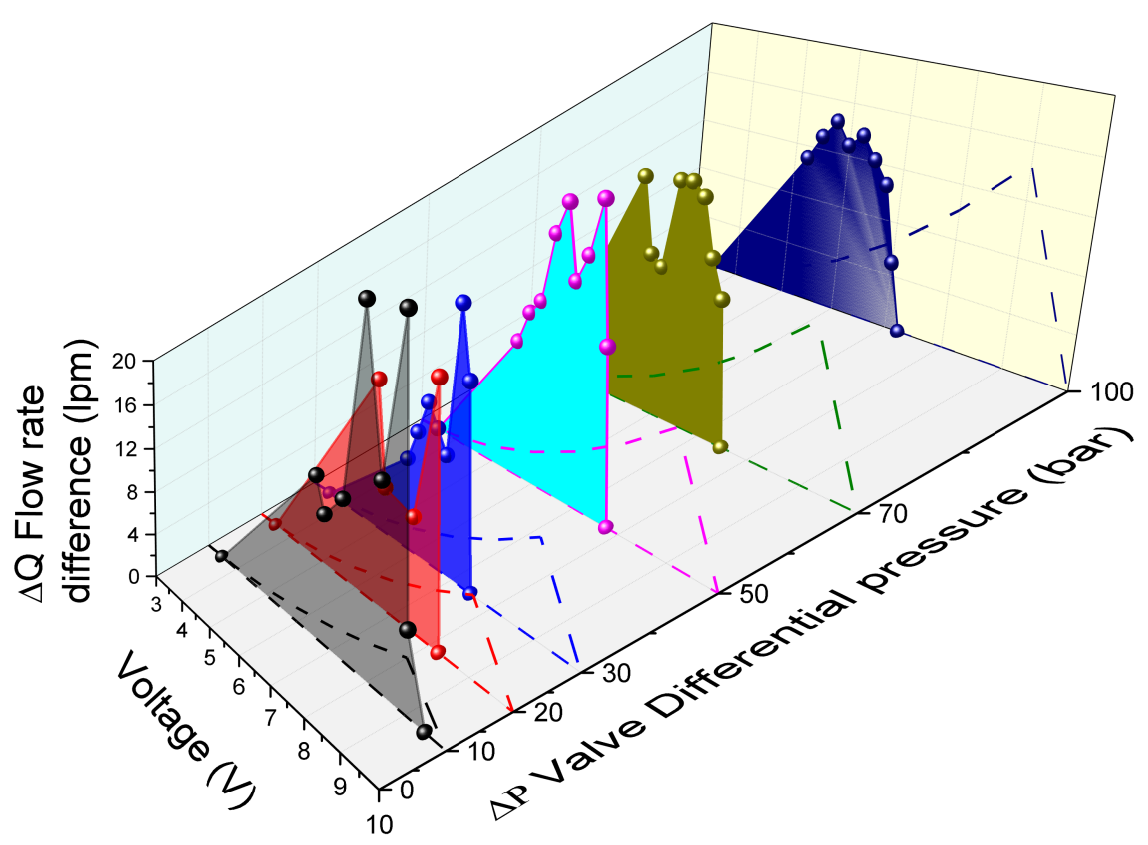

Figure 5.13: Flow rate difference due to hysteresis at 10/20/30/50/70/100 bar differential pressure. Dash lines represent manufacturer's data and the solid lines show experimental results.

plotting multiple flow rate curves at various differential pressure profiles.

When the flow rate profiles for the opening trend are derived, it is necessary to derive the downward trend taking into account that the manufacturer's data indicates the hysteresis of $3 \%$. During this calculation, it should be noted that the maximum opening (flow rate) for both of the trends is the same. Hence the maximum flow rate difference between the trends can be observed in the opening of $80-90 \%$. The easiest way to show the influence of the hysteresis on the valve output is to calculate the flow rate difference between the trends. Based on this calculated difference (manufacturer's data) it can be plotted against the experimental flow rate difference - Fig. 5.13 .

The experimental and manufacturer's data discrepancies show that they are significantly different from each other. The HPU flow rate output limitation is $145-150 \mathrm{lpm}$ which means that the maximum opening of the valve shifts from $10 \mathrm{~V}(100 \%)$ down to $6-9 \mathrm{~V}$ (depending on the differential pressure across the valve). Even though it was not possible to achieve $100 \%$ valve opening experimentally, the plot still shows a significant difference in the flow rate. It also proves the point that the hysteresis effect in this tested valve is stronger than it was predicted with the use of the manufacturer's data.

The hysteresis effect means that a motor (and any actuator) controlled by 
the valve, will not behave in the same manner during opening and closing. Therefore, it is necessary to implement a hysteresis compensation algorithm into its control system. Meaning that the valve should be able to achieve the same flow rate (hence the actuator output) regardless of the residual magnetisation of the valve's coil. In the case of the system presented in this research, it is feasible to use the motor's velocity as the reference that should be achieved regardless of the hysteresis. This means that the system should be able to decrease the control voltage output to the valve, to reduce its orifice area, resulting in lowering the flow rate, to achieve the required output from the drive train.

\subsubsection{Hydraulic effects in the hydraulic circuit}

The dynamics of the hydraulic fluid also depend not only on its chemical but physical properties. In case of rapidly accelerated systems, it means that laminar and turbulent realms might affect the behaviour of the hydraulic drivetrain. It should be noted that the use of different fluids might change the system outputs as well. Fluids are mainly categorised into Newtonian and nonNewtonian fluids. Mineral oil and water-glycol blend are both non-Newtonian fluids that can be classified as non-compressible fluids [71, 119].

One of the most critical parameters that can categorise the liquid flow realm (hence the flow behaviour) is Reynold's number. The number is calculated differently for pipes, valves, and different geometric orifices. The geometry also can influence the flow coefficient of the valve (valve sizing coefficient). The experimental testing of the valve showed a classic example of a differential pressure dip across the valve. The magnitude of the "vena contracta" dip is determined by the poppet's geometry. In our particular case, this can potentially change the acceleration behaviour of the system [10] - Fig. 5.14. This corresponds to $F_{L}$ coefficient and determines the likelihood of cavitation after the valve.

Valve Reynold's number can be calculated as:

$$
N_{R E V}=\frac{N_{4} \cdot F_{d} \cdot Q \cdot S G}{N_{o} \cdot \mu \sqrt{F_{L} \cdot C_{V}}}
$$

where $F_{d}$ is the valve style modifier, $\mathrm{Q}$ is the volumetric flow rate in $m^{3} / h r$, $\mu$ is the absolute viscosity, $F_{L}$ is the liquid pressure recovery factor, $C_{V}$ is the flow coefficient of the valve, and $N_{4}$ and $N_{o}$ are the units correlation constants.

For Reynold numbers smaller than 56, the flow realm is considered laminar and for values larger than 40,000 - turbulent. It should be noted that the majority of valve operations lie in the turbulent realm. 


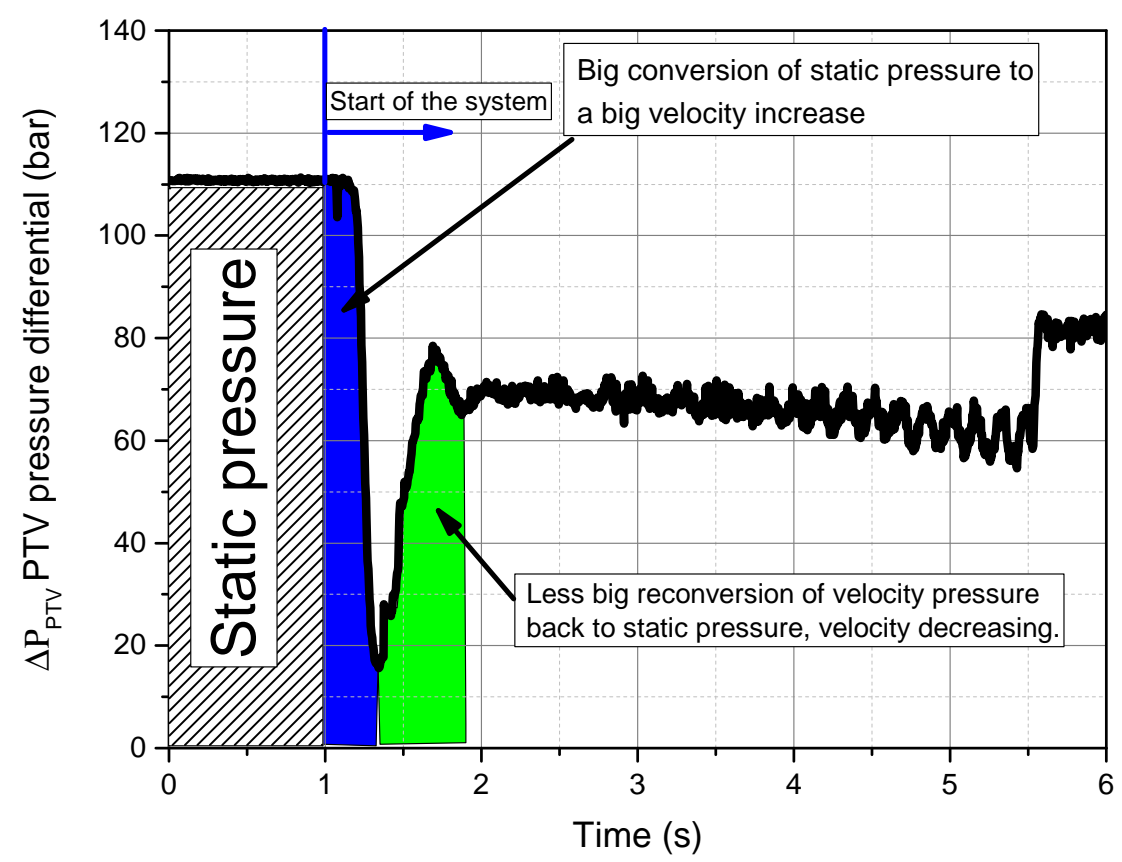

Figure 5.14: "Vena contracta" curve of the proportional valve TDA016 valve during the acceleration testing at the opening of $6.6 \mathrm{~V}$.

One critical factor that should be taken into account when a hydraulic system is designed is "cavitation". Cavitation is the process when the flow rate through the valve is not proportional to the square root of the pressure drop. When the flow rate is constant, it is described as a "choked flow". Cavitation is caused by pressure conditions, not the flow rate.

\subsubsection{Hydraulic nonlinearities in the drive train}

Taking into account that the manufacturer, Parker, provides only a 10 bar flow rate characteristic for the studied valve, it is necessary to calculate and plot the flow rate profiles at different differential pressure profiles. It is achievable through the calculation of a flow coefficient of the valve, remembering that:

$$
\begin{aligned}
& Q=f(i, \Delta P) \\
& Q=C_{v} \sqrt{\frac{\Delta P}{S G}}
\end{aligned}
$$

where $Q$ is the flow rate in lpm, $C_{n u}$ is the flow coefficient, $\Delta P$ is the differential pressure (bar), SG is the specific gravity.

With the derived flow coefficient, it is possible to calculate and plot the required flow rate profiles of the valve by using the same equation [120]. By 


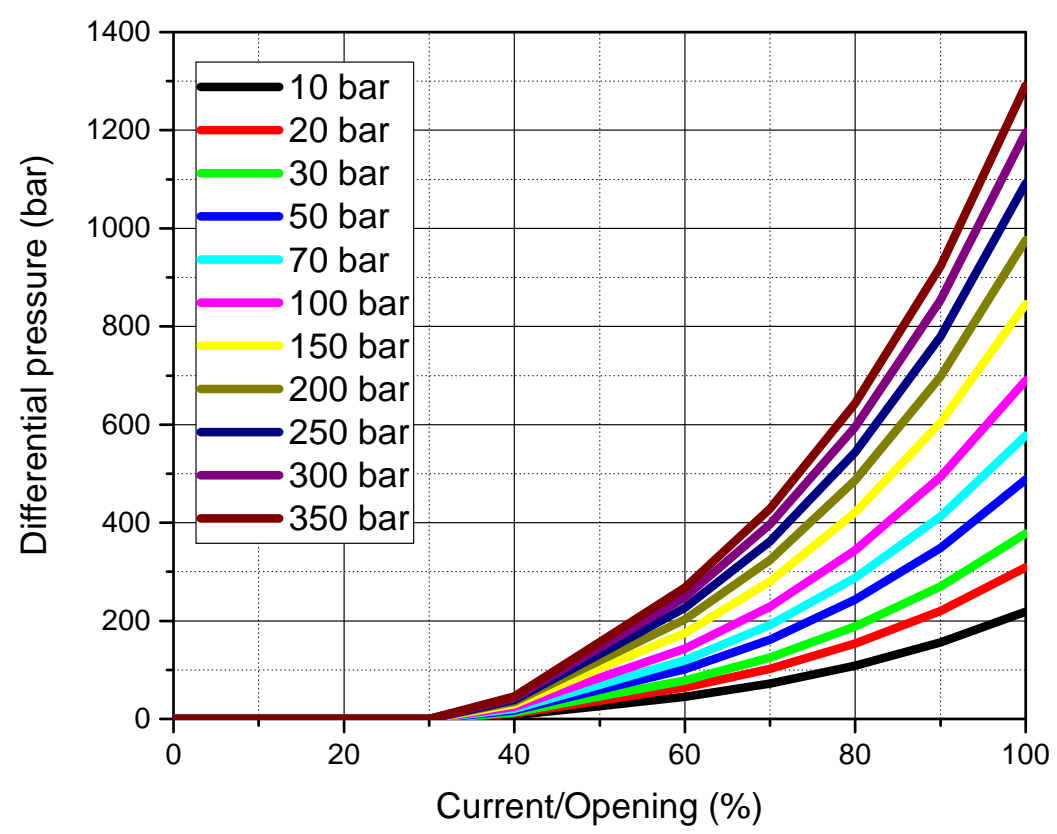

Figure 5.15: Derived flow rate profiles through the valve at different differential pressure profiles.

utilising the plotted curves, it is possible to calculate polynomials to implement this look-up table in its mathematical model. To achieve the highest accuracy, third order polynomials were utilised.

$$
Q=A \cdot x^{3}+B \cdot x^{2}+C \cdot x+D
$$

This relationship describes a single flow rate profile, showing the correlation between the opening and the flow rate. To make this system dependable on the differential pressure, it is necessary to find a mathematical relationship between the coefficients of the flow rate polynomials. It can be done by plotting all the values of A, B, C, and D, as it is presented in Fig. 5.16.

By utilising this correlation, it is possible to find the relationship between these values and the differential pressure across the valve.

$$
\left\{\begin{array}{l}
A \\
B \\
C \\
D
\end{array}\right\}=\left\{\begin{array}{c}
-5 \cdot 10^{-9} \cdot \Delta p^{2}+5 \cdot 10^{-6} \cdot \Delta p+0.0002 \\
-1 \cdot 10^{-7} \cdot \Delta p^{2}+9 \cdot 10^{-5} \cdot \Delta p+0.004 \\
7 \cdot 10^{-6} \cdot \Delta p^{2}-0.0056 \cdot \Delta p-0.2414 \\
-2 \cdot 10^{-5} \cdot \Delta p^{2}+0.013 \cdot \Delta p+0.5574
\end{array}\right\}
$$

By putting these polynomials into the original flow rate equations, it is possible to establish the relationship with the flow rate, differential pressure across the valve, and the opening of the valve. 


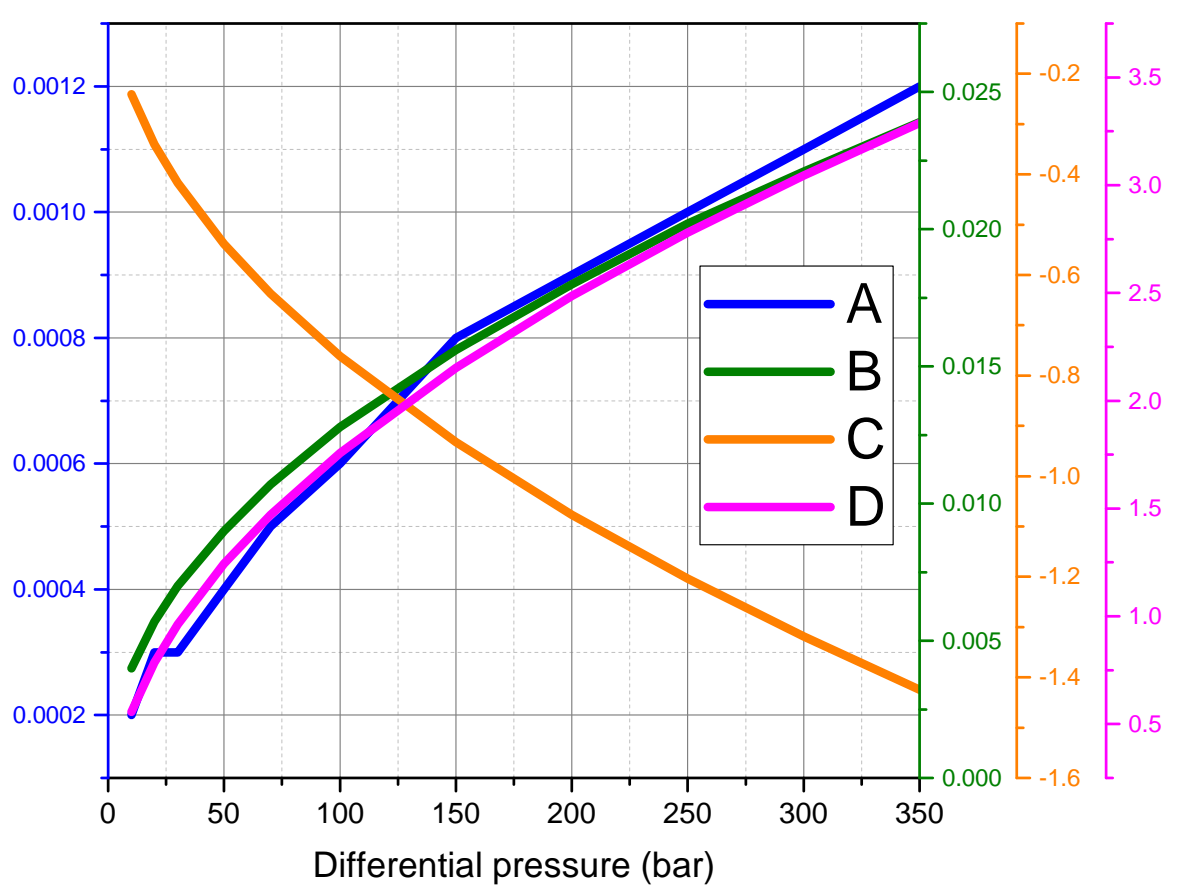

Figure 5.16: Coefficients of the flow rate polynomial

From the aforementioned correlations, it can be speculated that the flow rate calculation and its prediction is a straightforward process. However, in real life, there are a few variables that affect the flow rate profiles.

Referring to the flow coefficient equation, we can specify that

$$
Q=f\left(C_{v}, \Delta P, S G, x\right)
$$

where $Q$ is the flow rate in lpm, $C_{n u}$ is the flow coefficient, $\Delta P$ is the differential pressure (bar), SG is the specific gravity.

Remembering our power stage experimental testing, it is reasonable to assume that the differential pressure across the valve during the testing is constant, meaning that it does not change the tested flow rate curve (for instance, $\Delta \mathrm{P}=70$ bar). However, within this flow rate:

$$
Q=f\left(C_{v}, S G, x\right)
$$

where $Q$ is the flow rate in lpm, $C_{n u}$ is the flow coefficient, $\Delta P$ is the differential pressure (bar), SG is the specific gravity.

As we mentioned and confirmed above, the flow coefficient $\left(C_{v}\right)$ of the valve depends on the opening of the valve $x$. The next variable in the function expression the specific gravity (SG) of the mineral oil in the main hydraulic 
circuit. It should be noticed that the SG can be calculated as:

$$
S G=\frac{\rho_{\text {mineraloil }}}{\rho_{\text {reference }}}
$$

where $\rho_{\text {mineraloil }}$ and $\rho_{\text {reference }}$ are the density of mineral oil and reference in $\mathrm{kg} / \mathrm{m}^{3}$ and SG is the specific gravity of the fluid.

$$
S G=\frac{\rho_{\text {oil }}}{\rho_{\text {water }}}=\frac{f(T, P)}{f(T, P)}=f(T, P)
$$

Both of the densities ( $\rho_{\text {mineraloil }}$ and $\rho_{\text {water }}$ ) depend on the temperature and pressure. Meaning that the fluid's temperature affects the flow rate in the hydraulic circuit as well:

$$
Q=f(T, x)
$$

The temperature of the fluid heavily depends on the ambient temperature and the system's operation, since fluid and mechanical friction affect the temperature as well.

With regards to the opening of the valve, this parameter only seems to be linear and constant in case of quasi-state tests. The valve opening depends on the current flowing through the valve's coil. The current from the central NI controller, NI-9068, is amplified by an amplifier making from $10 \mathrm{Vdc} / 20 \mathrm{~mA}$ the required $16 \mathrm{Vdc} / 1.05 \mathrm{~A}$. One of the main components of the amplifier is a trimpot. It was tuned to deliver stable $1.05 \mathrm{~A}$ corresponding to a $10 \mathrm{~V}$ output from the controller in laboratory conditions. However, it was noted that the maximum output current is not stable over time since the potentiometer is subject to temperature changes; hence its resistance varies. It results in:

$$
i=\frac{u}{r} \longrightarrow r=f(T, t)
$$

where $i$ is the coil's current (A), $u$ is the applied voltage to the coil (V), $r$ is the resistance of the coil (Ohm).

Meaning that:

$$
x=f(T, t) \Longrightarrow Q=f(T, t)
$$

This temperature-time relationship is exceptionally challenging to calculate. Even though the ambient temperature changes during the system operation, the fluid temperature changes depending on the current operation. This makes the prediction process of the temperature correlation and the flow rate in the hydraulic circuit extremely difficult to establish.

To further verify the non-linearity and complexity of the presented hydraulic circuit, a hydraulic analysis was performed in the software ANSYS 


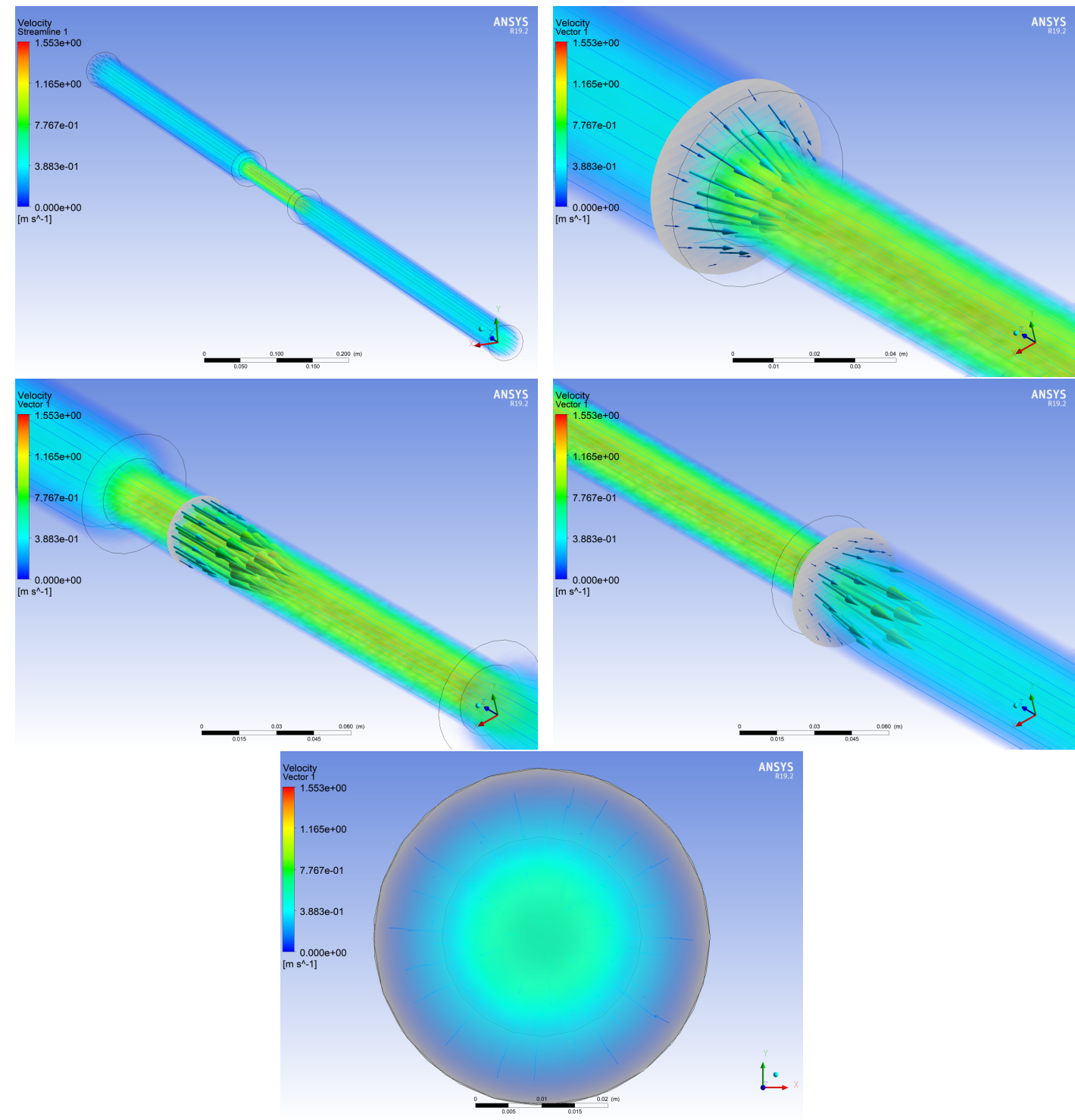

Figure 5.17: Fluid velocity distribution in the pipe connected to the control hydraulic valve TDA016.

19.2. Since a detailed hydraulic analysis of the main components of the system is out of the scope of this thesis, only a tube coming from the manifold to the control valve was simulated. According to the inlet pressure of 110 bar resulting in the fluid velocity of $0.3 \mathrm{~m} / \mathrm{s}$. The simulation plots are combined in Fig. 5.17. The presented simulations show how fluid flow is non-uniform, making it is difficult to predict and estimate the behaviour of the main hydraulic components in the circuit. 


\subsection{Chapter Summary}

This chapter presents the mathematical modelling and experimental verification tests of the proportional throttle valve (TDA016) employed in the prototype. This is achieved by utilising two different mathematical subsystems (control stage and power stage) along with the corresponding experiments.

The control stage third-order transfer function has been derived from the performed step response experimental testing at seven different voltage inputs. This process helped to ensure that the valve is modelled as realistically and accurately as possible within the utilised experimental setup. The power stage of the valve was modelled and experimentally characterised at six different pressure drops across the valve (limited by the maximum inline pressure from the hydraulic power unit).

This chapter discusses the hysteresis effect that is presented in the studied TDA016. This study showed that the experimental hysteresis is significantly larger than the nominal value (11-35\% and 3\% respectively). An overview of hydraulic effects in the central hydraulic circuit finalises the chapter. 


\title{
Chapter 6
}

\section{Hydraulic motor parameterisation}

\begin{abstract}
A hydraulic motor is the most commonly used hydraulic actuator, and is utilised in the current system for accelerating the synchronous generator. Its speed, torque, and acceleration are determined by the flow rate through the motor, which is determined by the opening of the TDA016. The main parameter that determines these characteristics is the displacement of the motor, which can be changed with the use of the employed swash plate drive. The main dynamic characteristics of the motor and the drivetrain, in general, are determined by the combined inertia of the drive. This aspect of the motor (drive) parameterisation is the most complicated due to the presence of the hydraulic brakes and the coupling on the shaft between the motor and the generator. To ensure the accurate mathematical representation of the inertia, it is required to perform the dynamic acceleration testing. The motor's displacement also can be verified only experimentally due to the inability to verify the current angle of inclination of the swash plate in the motor. The logical progression through the project steps is shown in Fig. 6.1.
\end{abstract}

\subsection{Signal processing and instrumentation}

In case of the presented research, the drivetrain's parameterisation starts with the displacement verification (it determines the maximum speed), and the combined inertia derivation, which determines the train's acceleration time [52]. For the verification of the displacement of the motor, the following equa-

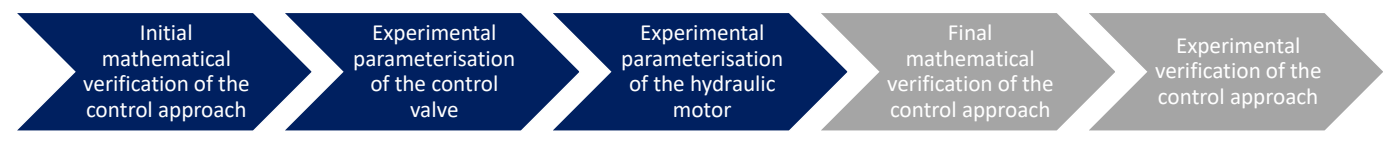

Figure 6.1: Steps for the system feasibility, focusing on the hydraulic motor characterisation. 


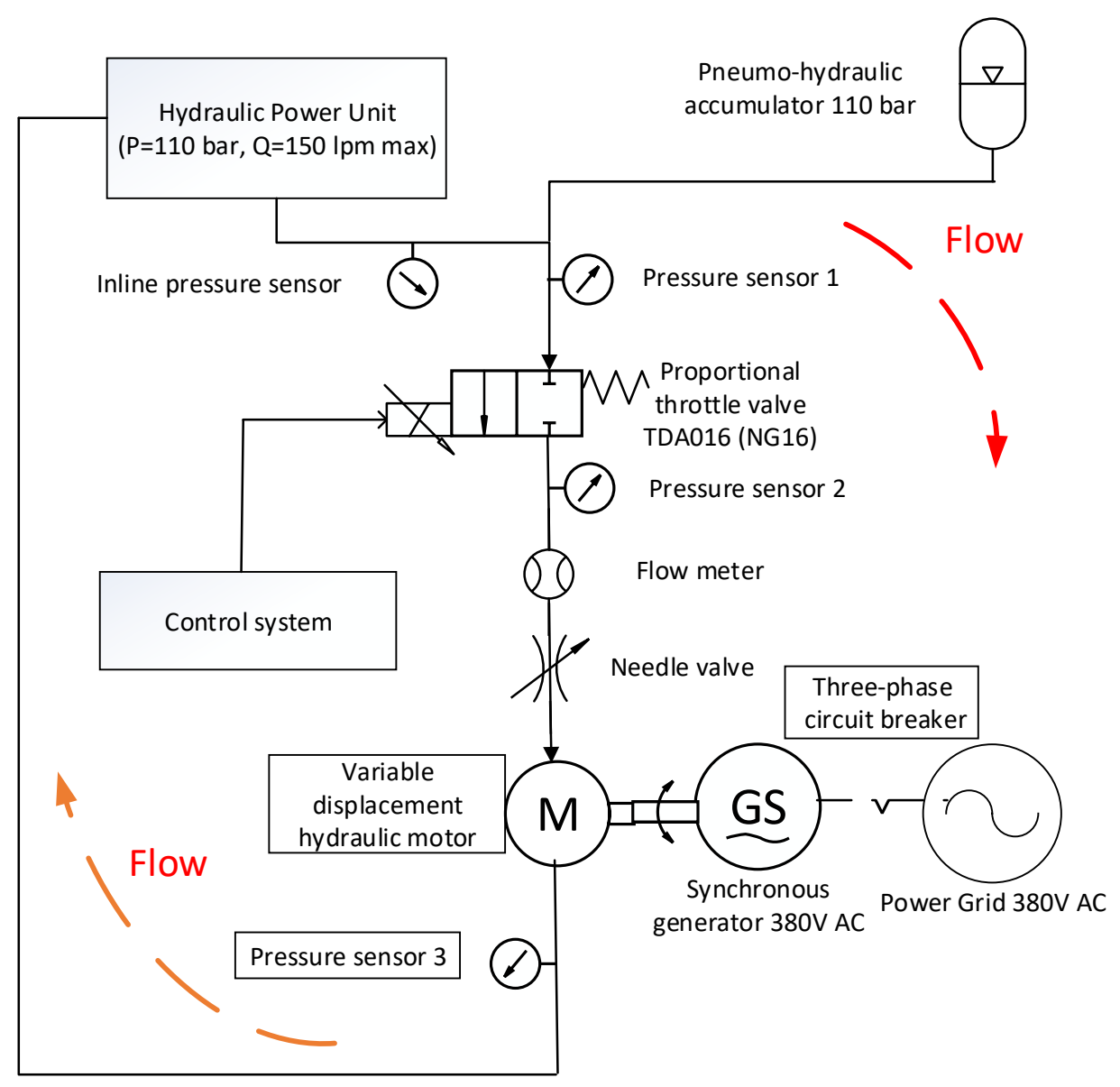

Figure 6.2: Schematic of the data acquisition system utilised for the experimental testing of the hydraulic motor (drivetrain).

tion can be employed:

$$
V_{D}=\frac{Q}{N_{R P M}}
$$

where $N_{R P M}$ is the motor's velocity in $R P M, Q$ is the flow rate through the motor in $l p m$, and $V_{D}$ is the motor's displacement in $l / r e v$.

To measure the position and velocity of the generator, the quadrature encoder installed on the rotor's shaft was utilised. Its output was measured by the digital input module NI-9401 on the controller chassis. An overview of the utilised DAQ system for this testing is presented in Fig. 6.2.

Some of the NI digital input modules (including NI-9401) have speciality digital configuration - quadrature input mode. In this regime, the module outputs the position and velocity channels based on the values of three channels 0,1 , and 2 which correspond to the A, B, and index channels of the quadrature encoder. The position measurement units are counts and velocity is measured in counts per second. The next step is to convert them into a position in radi- 
ans:

$$
\theta=\frac{K_{\text {count }} \cdot 2 \pi}{\text { Encoder }_{C P R}}
$$

where $K_{\text {count }}$ is the current position of the encoder and Encoder ${ }_{C P R}$ is the encoder count per revolution (10000 according to the manufacturer's data with the counting mode $\mathrm{X} 4$, it gives 40000 counts per revolution - the current resolution of the encoder).

Velocity measurements are carried out by the programmable function interface counter on the cRIO board - the same principle as with the position measurements. The output is in counts/time intervals and to convert it into $\mathrm{rad} / \mathrm{s}(\omega)$ or rev/s $\left(N_{R P S}\right)$ the following equation should be used:

$$
\omega=\frac{K_{\text {countPerInt }} \cdot 2 \pi}{\text { Encoder }_{C P R} \cdot x \cdot 10^{-6}}
$$

The maximum output speed is:

$$
N_{R P S}^{\max }=\frac{\text { Freq }_{\max }}{\text { Encoder }_{\text {resolution }}}=\frac{600000}{10000}=60 \mathrm{rev} / \mathrm{s}
$$

where Freq $_{\max }$ is the maximum output frequency $(\mathrm{Hz})$.

Taking into account that the maximum velocity of the generator is $25 \mathrm{rev} / \mathrm{s}$ (1500 RPM - $50 \mathrm{~Hz}$ ) or $30 \mathrm{rev} / \mathrm{s}(1800 \mathrm{RPM}-60 \mathrm{~Hz})$, this digital input module is suitable for the current measurements.

To measure the displacement of the motor, its velocity should be obtained via the encoder that is mounted on the drivetrain's shaft, and the flow rate can be acquired by the flow meter. The first run of the drive showed that the displacement was at the value of $149.5 \mathrm{cc} / \mathrm{rev}$. This is a critical factor for hydraulic drives with limited inline flow rate. In the case of the presented system, the electro-hydraulic drivetrain should be accelerated up to 1500 RPM $(50 \mathrm{~Hz})$ or $1800 \mathrm{RPM}(60 \mathrm{~Hz})$ to synchronise the generator with a power grid. Meaning that the displacement of the motor should be adjusted in the way, that the system can be accelerated to the required velocity (frequency in our case) even at the flow rates $<150 \mathrm{lpm}$. To achieve this, the motor's displacement was decreased to $71.5 \mathrm{cc} / \mathrm{rev}$.

Another unknown variable of the electro-hydraulic drivetrain is its combined inertia. It is a crucial factor that determines how quickly the drive can accelerate to the specified speed.

$$
J=T_{m e c h} /\left(\frac{d \omega}{d t}\right)
$$

where $\mathrm{J}\left(\mathrm{kg} / \mathrm{m}^{2}\right)$ is the combined inertia of the motor and the generator, $d \omega / d t$ $\left(\mathrm{rad} / \mathrm{s}^{2}\right)$ is the motor's acceleration, and $T_{\text {mech }}$ is the drive train's torque in $N \cdot m$. 
Overall, it is possible to use torque sensors to measure the drivetrain's inertia. The drivetrain discussed in this research was not equipped with them; hence it is required to estimate the drivetrain's torque mathematically, since:

$$
T_{\text {mech }}=\frac{\eta_{o} \cdot \Delta P \cdot Q}{N_{R P M}}=\frac{\eta_{o} \cdot \Delta P \cdot V_{D}^{c c}}{20 \pi}=\frac{\eta_{o} \cdot \Delta P \cdot V_{D}^{c c}}{62.83}
$$

where $\eta_{o}$ is the overall efficiency, $\Delta P$ is the differential pressure across the motor in bar, $V_{D}$ is the volumetric displacement of the motor in $c c / r e v$. The efficiency of the motor depends on its speed and the pressure across it, so the exact efficiency can be found by using the corresponding manufacturer's data.

The last unknown variable in the inertia calculation $(6.5)$ is the acceleration of the electro-hydraulic drive. However, it is possible to measure this with the installed encoder.

\subsection{Drive train acceleration measurement}

In order to find the motor/generator's acceleration a difference between the previous velocity measurement value and the current value for the time interval should be used:

$$
\alpha_{g e n}=\frac{d \omega_{g e n}}{d t}
$$

where $\alpha_{\text {gen }}$ is the acceleration in $\mathrm{rad} / \mathrm{s}^{2}$ and $\omega_{\text {gen }}$ is the generator speed ( $\mathrm{rad} / \mathrm{s}$ ).

To find this speed difference over a period, a structure with shift registers can be used. The dynamic measurements (speed and position of the generator) are obtained under high resolution $(4 \mathrm{MHz})$ and processed through the main (1 KHz) controller loop [69].

The acceleration channel is measured directly in this loop, which means that the high-resolution velocity channel is downsampled and divided by the loop execution time. This results in digitising of the acceleration measurement channel, as it can be seen in Fig. 6.3.

To get feasible measurements for the inertia calculations (and logging) and also to use this signal for the main controller operation, it is necessary to filter the measurement channel. During the testing of the prototype, there were two tested techniques - the mean and Savitzky-Golay filtering [102], as both of them are presented in Fig. 6.3. Other filtering techniques available in Labview did not improve the acceleration output and were too slow to perform in the scan mode. It can be noticed the magnitude of the filtered signal is lower than the original measurements. Hence, to have trustworthy results for the filtered acceleration measurement channel, it is required to calibrate the filter's settings and related coefficients. Another significant point that should be considered is 


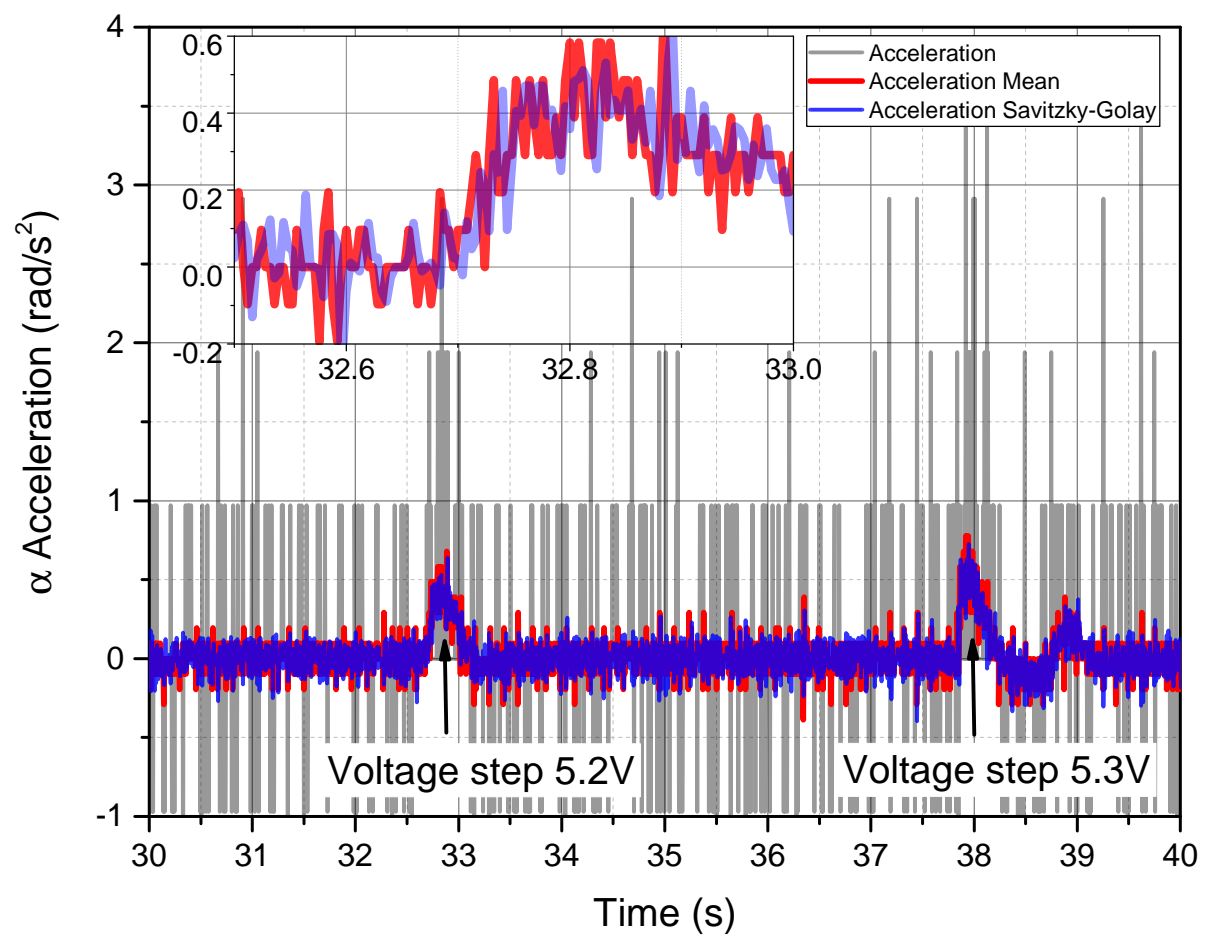

Figure 6.3: Acceleration channel measurement with two step responses - experimental results.

the sample window length that should be used for the chosen filtering technique. Due to the computational burden on the main controller, the decision was made to use Savitzky-Golay filtering over the mean option. Comparison of this technique with different window lengths is presented in Fig. 6.4.

It is imperative to model the above-described filtering techniques by employing a mathematical model. As an example of the filtering techniques, Savitzky - Golay filtering has been chosen with the following sample windows - Fig. 6.5. It helps to verify that the time delays in the experimental setup and the model are the same. From this perspective, there is a minor difference in the magnitude and delay of the filtered outputs. It should be noted that the same behaviour of the filtered signals can be observed with the use of the Simulink model with added moving average filters. The Savitzky-Golay filtering technique has been implemented in the hardware with a window length of 10.

The next step in the verification of the acceleration measurement channels is the calibration of the signal to achieve the realistic magnitudes of the filtered signal. The initial magnitude of the acceleration output does not drastically affect the synchronisation time. It's mainly determined by the quasi state ac- 


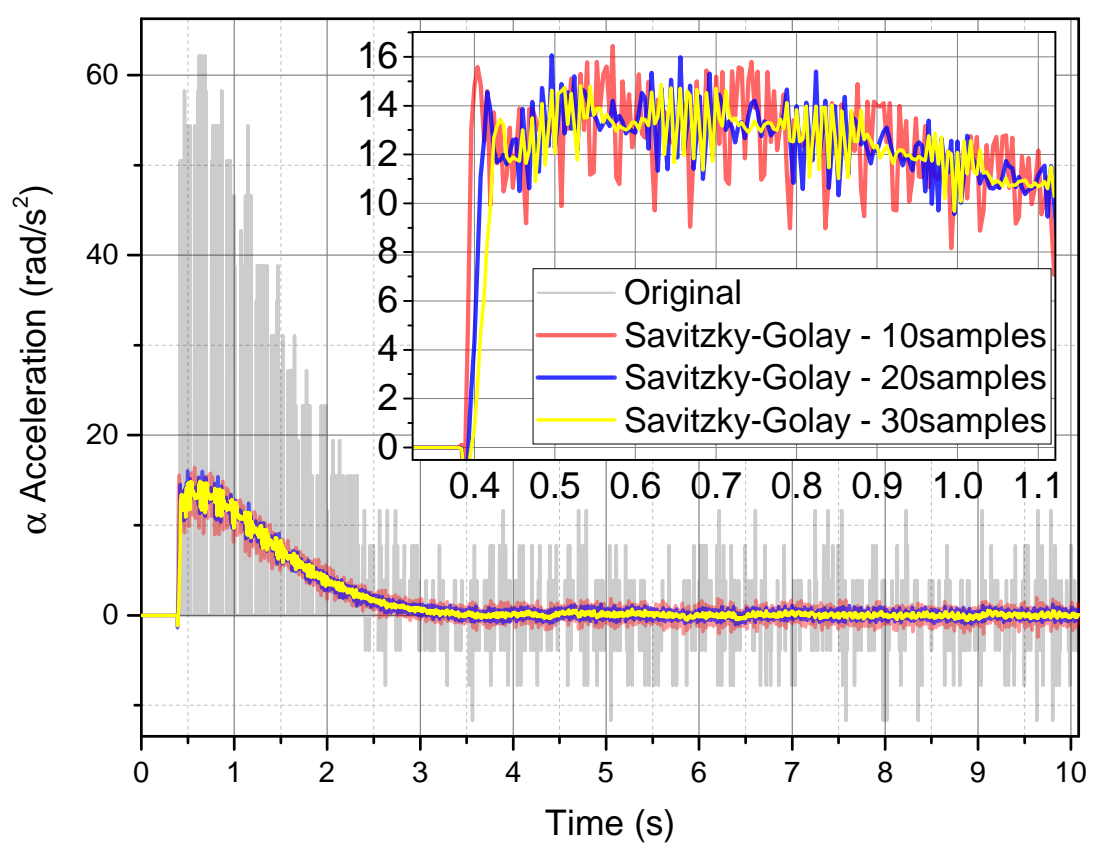

Figure 6.4: Acceleration comparison of the original and filtered signals with different sample length windows - Experimental results.

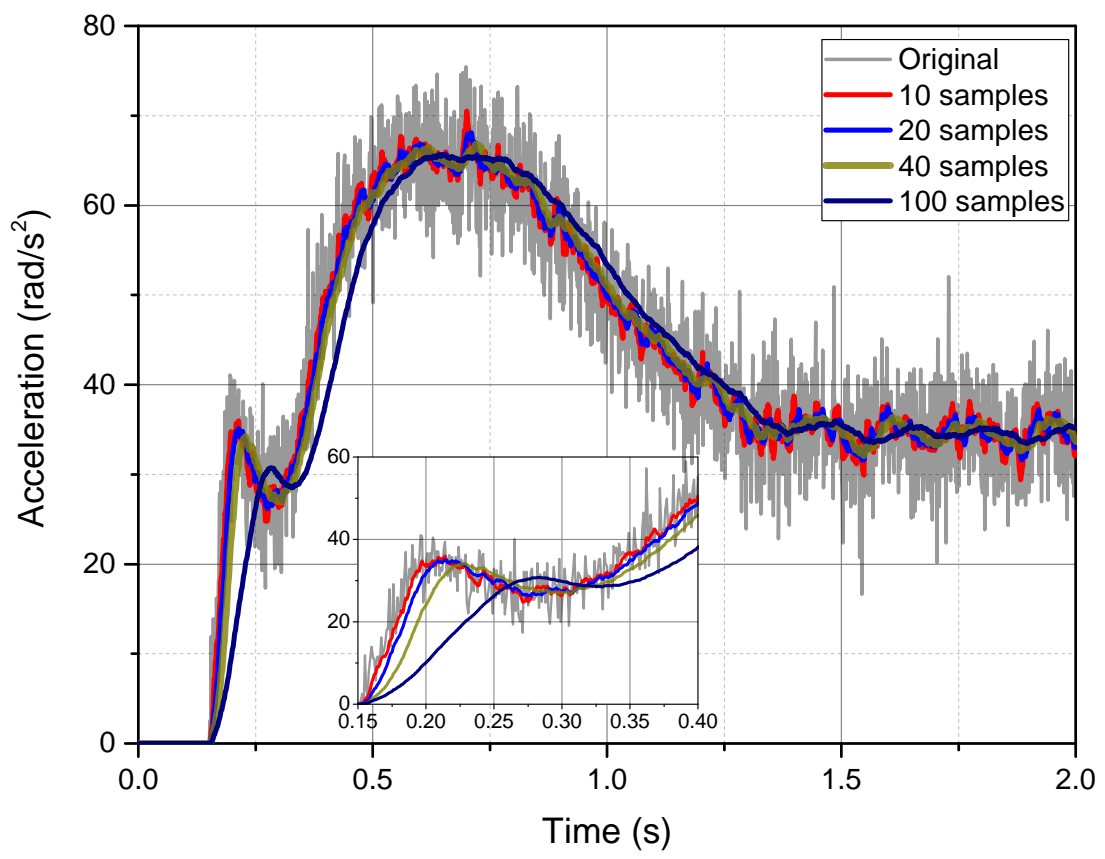

Figure 6.5: Acceleration comparison with different Savitzky - Golay filters in Labview with the logged data from the Simulink model. 


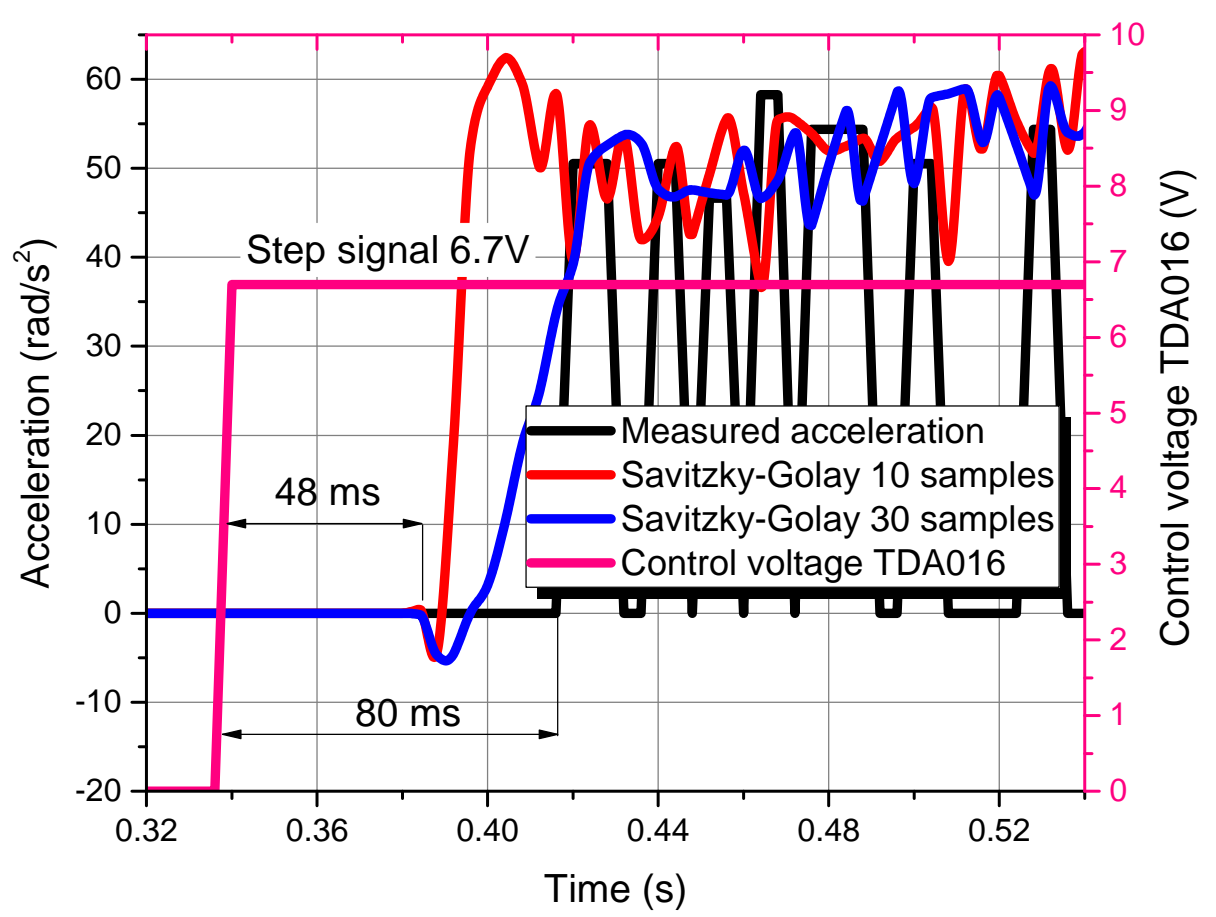

Figure 6.6: Experimental acceleration channel measurement - calibration of the signal with Savitzky-Golay filtering.

celeration measurement. To achieve the realistic values of the filtered acceleration output, a gain function was used. This helped eliminate the difference between the actual acceleration values and filtered output. Thus, it is possible to achieve the following plotted results (plotted only for the Savitzky-Golay filtering technique) - Fig. 6.6. It should be noted that in this case, it is possible to verify the accuracy of the presented approach. This is a direct comparison of the magnitude of the original aliased acceleration signal with the calibrated filtered signal. Even though the original signal is digitised, the magnitude of it is still realistic.

After the calibration process finished, this measurement channel was used for the final verification of the drive's inertia, according to:

$$
J=T_{m e c h} /\left(\frac{d \omega}{d t}\right)
$$

where $J$ is the inertia of the drivetrain $\left(\mathrm{kg} / \mathrm{m}^{2}\right), T_{\text {mech }}$ is the torque output of the drivetrain $(N \cdot m)$ and $d \omega / d t$ is the drivetrain's acceleration $\left(\mathrm{rad} / \mathrm{s}^{2}\right)$.

The experimentally derived inertia is $1.6 \mathrm{~kg} / \mathrm{m}^{2}$.The inertia value was implemented in the MATLAB/Simulink model for further testing of the prototype. 


\subsection{Inertia calculation of the drivetrain}

With the calculated torque values and measured filtered acceleration it becomes possible to estimate the overall inertia of the electro-hydraulic drivetrain:

$$
J=T /\left(\frac{d \omega}{d t}\right)
$$

where $J$ is the inertia of the drivetrain $\left(\mathrm{kg} / \mathrm{m}^{2}\right), T_{\text {mech }}$ is the torque output of the drivetrain $(N \cdot m)$ and $d \omega / d t$ is the drivetrain's acceleration $\left(\mathrm{rad} / \mathrm{s}^{2}\right)$.

This equation was implemented in the main Labview code; however, there are other options for the aforementioned inertia verification. With the use of the logged acceleration data, and knowing the differential pressure profile (from the installed pressure sensors), the inertia can be calculated manually. It can be reasonable to do so if it is required to reduce the overall number of mathematical calculations in the main code (scan mode operation). Hence it is possible to use only two logged profiles to estimate the inertia of the system.

The inertia value has been implemented in the MATLAB/Simulink model for the further testing of the prototype. A step response test up to $6.6 \mathrm{~V}$ was performed to verify the train's dynamics with the output depicted in Fig. 6.7 .

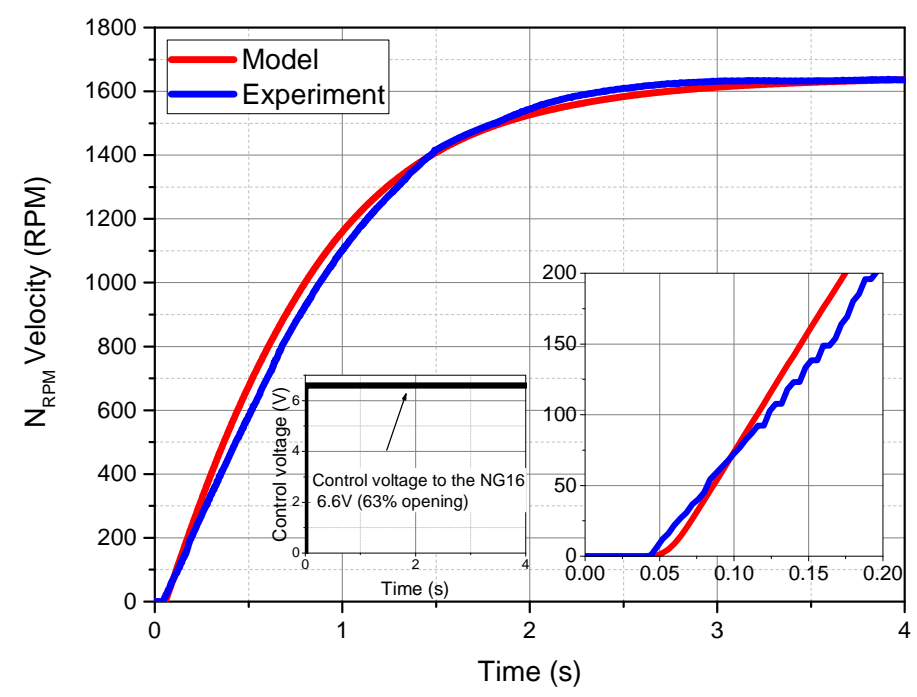

Figure 6.7: Velocity comparison of the motor - step test at 6.6V. Experimental set up and the mathematical model.

It can be seen that the initial delay of the motor and its model is the same, which proves the statement that the inertia of the drive has been verified correctly. During the stable region after $3 \mathrm{~s}$, the velocities are the same, which 


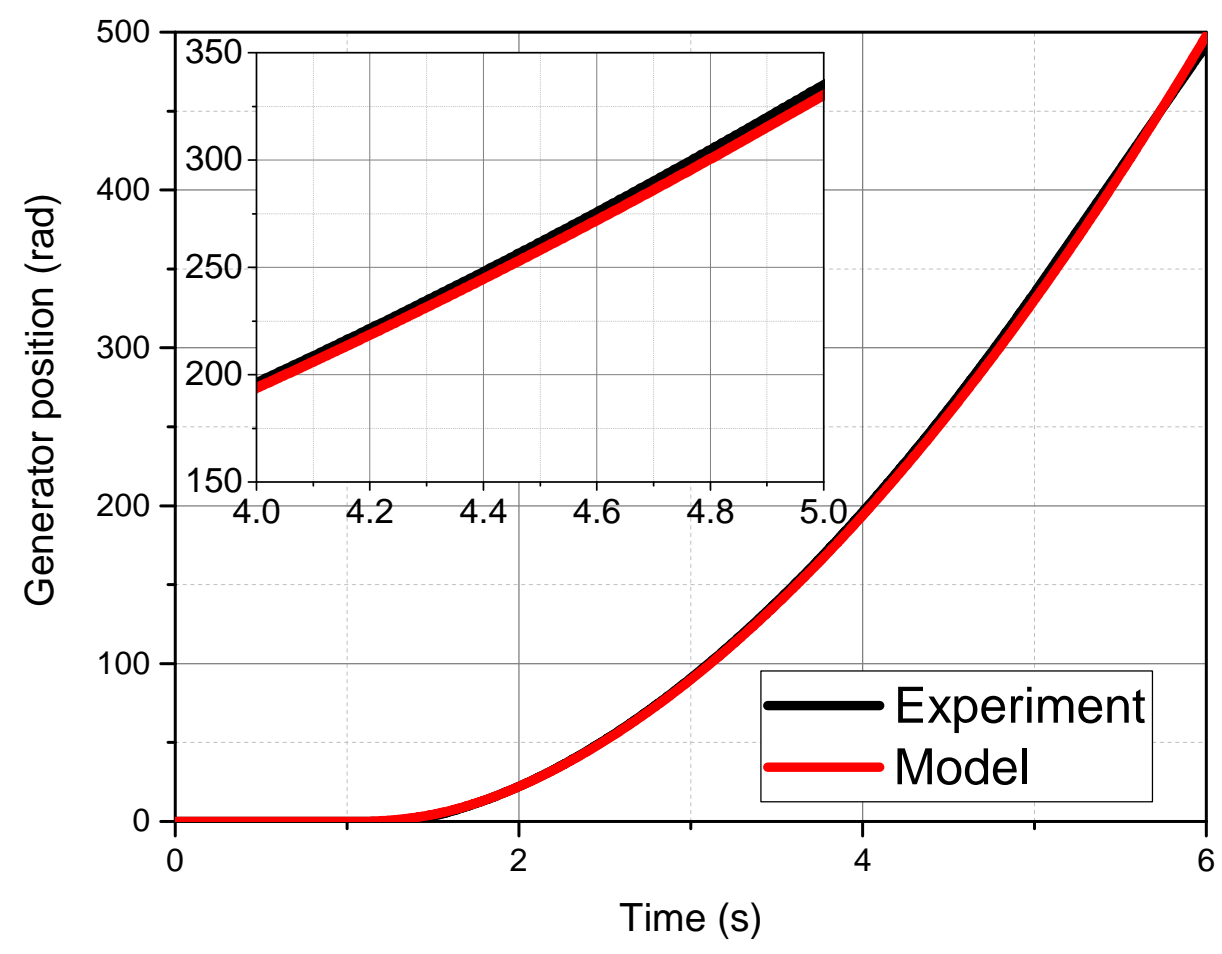

Figure 6.8: Comparison of the dynamics of the synchronous generator between the model and the hardware. Comparison of the generator position - model and experiment.

confirms the accurate derivation of the displacement. The only difference between these two velocity profiles is the initial acceleration stage at $0-1.5 \mathrm{~s}$. This difference is caused by the model of the control valve, mainly the control stage transfer function.

In this case, the generator phase profiles can be compared in order to establish a difference between the simulated results and the prototype that is shown in Fig. 6.8. These plots are close to each other depicting that the dynamics of the motor in the model are simulated quite accurately.

To summarise, the engineered mathematical model is proven to be acceptably accurate, meaning that it can be used for the system's stability testing. These tests are too dangerous to perform experimentally since the prototype is pressurised. Another way to employ this model is to test the relationship between the hydraulic fluid's temperature and the dynamics of the hydraulic drivetrain. This testing is difficult to perform since the temperature of the fluid and the components of the main hydraulic circuit are changing during the testing. Since it is difficult to create such an environment for these tests, the mathematical model can be used to study this correlation. 
Taking into account that the dynamics of the drivetrain might change due to temperature changes, the primary control system has to adjust its outputs to keep the train within its stability boundaries and perform accurately. By and large, any changes in the behaviour of the drivetrain impose an extra burden on its control system and/or the central controller. The following chapter can help to establish the significance of the temperature on the components of the electro-hydraulic drivetrain.

After the parameterisation of the main components of the system and calibration of all inputs and outputs in the Labview code, it is possible to perform synchronisation tests on the real system.

\subsection{Chapter Summary}

This chapter gives an overview of the mathematical modelling and experimental characterisation of the variable-displacement hydraulic motor utilised in the system. The process starts with the explanation of the dynamic's measurements (position and velocity) via the incremental encoder mounted on the drivetrain's shaft. The first motor parameter that was verified with the use of the implemented DAQ systems is the motor displacement. Taking into account the limited inline flow rate from the hydraulic power unit, the displacement was decreased from $141.5 \mathrm{cc} / \mathrm{rev}$ to $71.5 \mathrm{cc} / \mathrm{rev}$. It allowed the generator to accelerate to the required synchronisation speed of 1500 RPM at the flow rate $<150$ lpm.

The acceleration measurements of the drivetrain were employed to derive the combined inertia of the setup. Since the acceleration channel is digitised, the mean and Savitzky-Golay filtering techniques were employed to get realistic acceleration values. A step response test was performed to compare the simulation and experimental dynamics of the train and verified the derived displacement and inertia values. 


\section{Chapter 7}

\section{Demonstration and Evaluation of the Synchronisation Approach}

The mathematical model was updated after the experimental verification of the primary system components. It helped to bring the simulation results to the experimental system outputs as close as possible, before the first experimental testing of the synchronisation approach took place (April 2018).

Since the primary application of the proposed system is a FIR unit, its synchronisation time should be within 1-6 s after the unit is triggered. However, for the experimental control system verification, the decision was made to more gradually accelerate the generator and synchronise it within 4-5 s. The slower acceleration gives more time for a machine operator to manually decrease the generator speed by using the emergency hydraulic brakes and/or the main NI controller. The prototype was tested according to the procedure highlighted in Fig. 7.1 .

Bearing in mind the proposed slow acceleration experimental testing, this chapter is structured as follows:

1. Calibration of the electrical components of the hardware built.

2. Tuning of the central controller of the prototype for the slow acceleration testing.
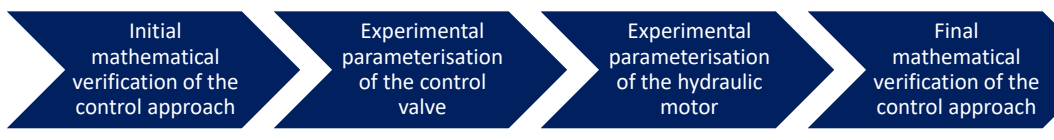

Figure 7.1: Step by step verification of the synchronisation approach of the hardware built with the focus on the control approach experimental verification. 
Table 7.1: NI9263 Accuracy

\begin{tabular}{|l|l|l|l|}
\hline \multicolumn{2}{|l|}{ Measurement Conditions } & $\begin{array}{l}\text { Percent of Reading } \\
\text { (Gain Error) }\end{array}$ & $\begin{array}{l}\text { Percent of Range } \\
\text { (Offset Error) }\end{array}$ \\
\hline \multirow{2}{*}{ Calibrated } & Maximum (-40 C to 70 C) & $0.35 \%$ & $0.75 \%$ \\
\cline { 2 - 4 } & Typical (25 C, \pm 5 C) & $0.03 \%$ & $0.1 \%$ \\
\hline \multirow{2}{*}{ Uncalibrated } & Maximum (-40 C to 70 C) & $2.2 \%$ & $1.7 \%$ \\
\cline { 2 - 4 } & Typical (25 C, \pm 5 C) & $0.3 \%$ & $0.25 \%$ \\
\hline
\end{tabular}

3. Experimental validation of the slow acceleration scenario.

4. Comparison of the experimental data and the simulated outputs for the verification of the model's accuracy.

5. Modelling of the fast acceleration scenario employing the realistic mathematical model.

6. Stability studies utilising the accurate mathematical model of the prototype.

\subsection{Calibration of the electrical components}

A complex multi-domain system (like the presented ss-CAES) is prone to various disturbances and instabilities. Hence, to assure the maximum accuracy of the system (bringing it as close to the mathematical model as possible), it is required to calibrate most of the components of the prototype to minimise any possible errors. Since the TDA016 is controlled via the NI9263 module, this module should be calibrated to be as accurate as possible.

It can be done through following the official procedure issued by National Instruments specifically for the 9263 modules (Table. 7.1). To ensure that the module meets the required specifications, it is necessary to use a $61 / 2$ digit DMM with voltage accuracy measurement of $\pm 40 \mathrm{ppm}$. By applying a voltage in the range of $\pm 10 \mathrm{~V}$, the measured voltage through the module should be recorded and then compared with the corresponding test values, with the results presented in Table.7.2 and Fig. 7.4(a).

Overall, the voltage module's measurements are standard for a calibrated module $( \pm 0.1 \%)$ and can't be improved.

A simulation run shows that this error introduced into the mathematical model as a constant error does not affect the synchronisation process of the system significantly as it is presented in Fig. 7.4 
Table 7.2: NI9263 Analogue Output calibration results

\begin{tabular}{|l|l|l|l|l|l|l|l|l|l|l|l|}
\hline \multicolumn{10}{|c|}{ NI9263 Calibration test } \\
\hline Control Voltage (V) & 0 & 0.25 & 0.5 & 0.75 & 1 & 1.25 & 1.5 & 1.75 & 2 & 2.25 & 2.5 \\
\hline Actual Voltage (V) & 0.00005 & 0.249211 & 0.499484 & 0.748996 & 0.999420 & 1.24911 & 1.49920 & 1.74915 & 1.99935 & 2.24925 & 2.49933 \\
\hline Control Voltage (V) & 2.75 & 3 & 3.25 & 3.5 & 3.75 & 4 & 4.25 & 4.5 & 4.75 & 5 & 5.25 \\
\hline Actual Voltage (V) & 2.74927 & 2.99949 & 3.24933 & 3.49968 & 3.74921 & 3.99998 & 4.24948 & 4.49997 & 4.74948 & 4.99985 & 5.24901 \\
\hline Control Voltage (V) & 5.5 & 5.75 & 6 & 6.25 & 6.5 & 6.75 & 7 & 7.25 & 7.5 & 7.75 & 8 \\
\hline Actual Voltage (V) & 5.49901 & 5.7492 & 5.99922 & 6.24911 & 6.49911 & 6.74914 & 6.99921 & 7.24918 & 7.49961 & 7.74915 & 7.99971 \\
\hline Control Voltage (V) & 8.25 & 8.5 & 8.75 & 9 & 9.25 & 9.5 & 9.75 & 10 & & & \\
\hline Actual Voltage (V) & 8.24941 & 8.50001 & 8.74961 & 9.00005 & 9.24997 & 9.50011 & 9.75012 & 10.00034 & & & \\
\hline
\end{tabular}

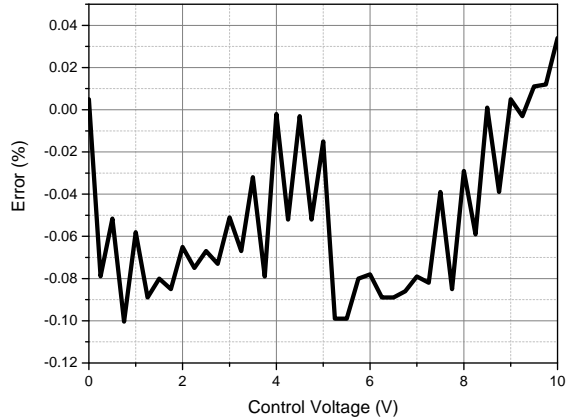

(a) Maximum voltage error.

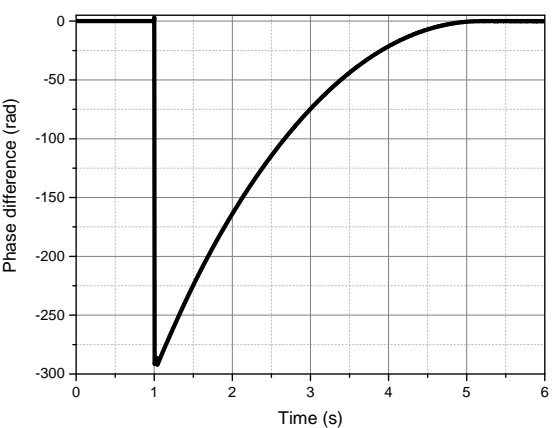

(b) Simulated phase difference.

Figure 7.2: Calibration process of the NI9263 Analogue Output module. a) Maximum recorded voltage error within the tested voltage range 0-10 V. (b) Simulated phase difference between the generator and the grid with the introduced voltage error $( \pm 0.1 \%)$ and at slow acceleration of the drive train.

Other potential disturbances in the system are not significant and will be studied later after the synchronisation approach is verified. The research presented in this section proves that despite having a constant error in the system (due to the NI9263 module voltage output), mathematically it is still possible to achieve synchronisation. Remembering this, the synchronisation approach should be verified by tuning the central controller. This study is presented further in the chapter.

\subsubsection{Sensitivity analysis of the control system}

One of the most complicated questions about the presented control system is the influence of the controller parameters on the system outputs, also known as sensitivity analysis. Two main factors determine successful synchronisation of the generator:

- The synchronisation time of the system, i.e., how fast the phase difference of zero is achieved.

- The value of the phase difference at the moment of synchronisation $\left( \pm 20^{\circ}\right)$. 


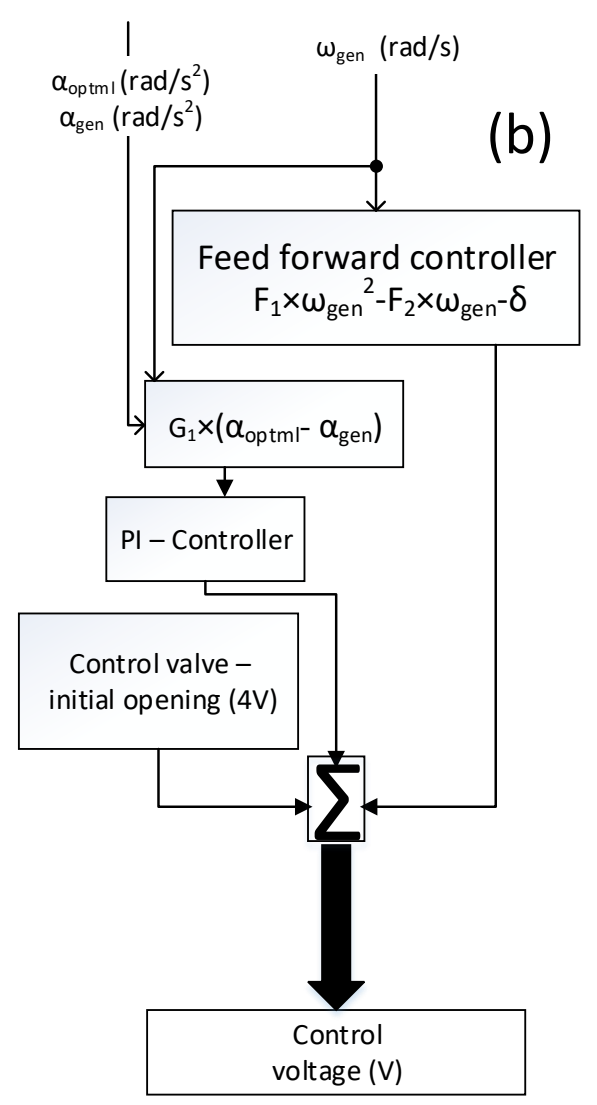

Figure 7.3: PI-controller block diagram in the main controller shown in Fig. 3.6.

There are two ways of analysing these dependencies. It is possible to change only the first parameter of the feedforward controller polynomial $\left(F_{1}\right)$. Another one is to change the ratio $F_{1} / F_{2}$ of the feedforward polynomial (see Fig. 7.3). The first option was performed during the experimental testing of the control system. Whereas the second option can be tested with the use of the realistic mathematical model. The results are depicted in Fig. 7.4.

It can be seen that regardless of which of the polynomial coefficients has been changed, the behaviour of the control system is the same. With the increased parameter, the synchronisation time increases, whilst the phase difference decreases. It is imperative to find the most suitable value that will maintain the phase difference within the synchronisation boundaries and at the same time, its synchronisation time is acceptable according to the current design of the system. It should be noted that it is possible to achieve the same synchronisation conditions with different values of $F_{1}$ and $F_{2}$. For which the output of the polynomial with new values should match the original output profile.

The main PI-controller has many different coefficients that can be tuned 


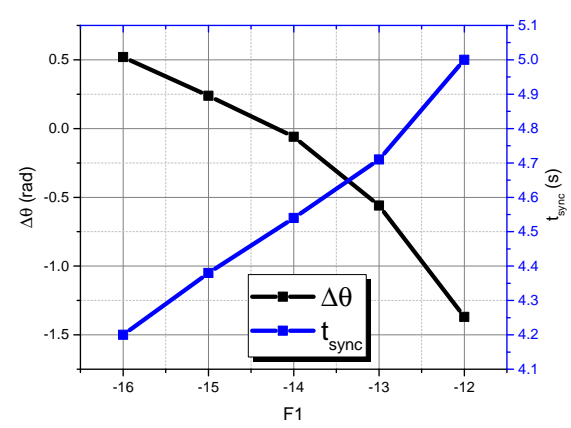

(a) Sensitivity analysis for F1 coefficient, F2=0.0034.

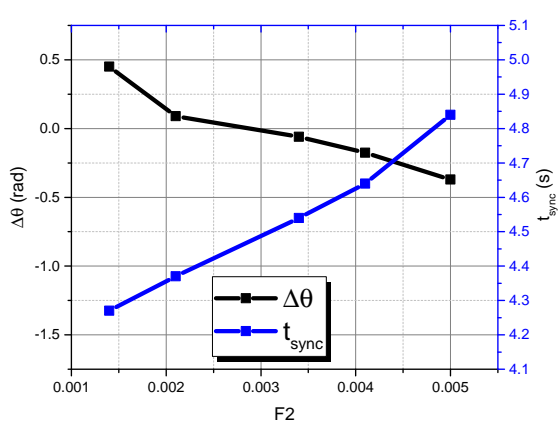

(b) Sensitivity analysis for F2 coefficient, $\mathrm{F} 1=-11.5^{-6}$.

Figure 7.4: Simulated sensitivity analysis based on two parameters of the controller - F1 and F2 in the feed forward system.

to reach synchronisation. Hence, if some of the coefficient's values are hard coded, it is still possible to reach a zero phase difference by tuning other controller parameters. It means that the control system is flexible concerning the tuning of the controller.

\subsection{Tuning of the hardware controller}

After modelling the slow acceleration scenario, the simulated coefficients of the controller were implemented in the hardware prototype.

The experimental set up follows the same acceleration algorithm and workflow as the model (Fig. 3.7), with some exemptions:

- Since there is no power grid, its phase and frequency were replaced with the corresponding signals from the mathematical model.

- The main controller code does not send a control signal to the circuit breaker, remembering that there is no power grid and the circuit breaker is not included in the tested circuit.

- The prototype is triggered at $1 \mathrm{~s}$ by inputting a trigger signal to the main acceleration derivation script.

The tuning process of the central controller is imperative for finding the most feasible operational boundaries. This can be done with the use of the implemented PI-controller, which is presented in Fig. 7.3.

When the system is undergoing calibration, it is required to set up the maximum allowable acceleration to the desired level (required synchronisation 
time). After specifying these parameters, the fine-tuning of the main controller can be performed by using one of three controller channels:

- Opening voltage of the valve. This is an offset of the valve control voltage which does not change and hence is constant at all times. This value should be sufficient to open the valve beyond the initial opening where the valve characteristics are highly non-linear. Through the valve's experimental verification, a value of $4 \mathrm{~V}$ was implemented into the control system.

- A PI-controller is used to provide feedback (zero-error) control of the acceleration channel (Fig. 7.3). It keeps the generator within the synchronisation boundaries in case of a sudden change of the grid's parameters and the generator dynamics (velocity and acceleration). The PI-controller was tuned by employing the mathematical model. Stable synchronisation and performance was achieved and then these coefficients were implemented in the hardware setup.

- Feed forward channel. When the valve is open, the differential pressure across it drops allowing the hydraulic fluid to flow through its ports [20]. It accelerates the electro-hydraulic drivetrain until it reaches the reference (grid) speed (frequency) for synchronisation. It results in a bigger pressure drop across the valve, however, according to the proposed approach, the generator's acceleration should be constant for synchronisation. It can be achieved at a constant pressure drop across the valve that should be constant regardless of the changes in the flow rate. Since there is a direct correlation between the flow rate in the system and the generator's speed, it is possible to use the velocity channel as the input for the pressure drop compensation system. Based on this assumption a feedforward subsystem was designed to keep the pressure drop across the valve constant while the motor accelerates - Fig. 7.3

It should be noted that the PI - controller was implemented in the hardware controller without any changes in comparison to the model. Even though it had been tuned in the mathematical model, it was still required to tune the control system in the hardware prototype. After the system is stabilised during acceleration tests, the related parameters were not changed. However, it is important to tune the control system to reach the required phase and frequency difference. Since it is not feasible to tune the PI - controller as it possibly can jeopardise the stability of the system, the decision was made to change the feedforward polynomial's coefficients. Since the feedforward polynomial 


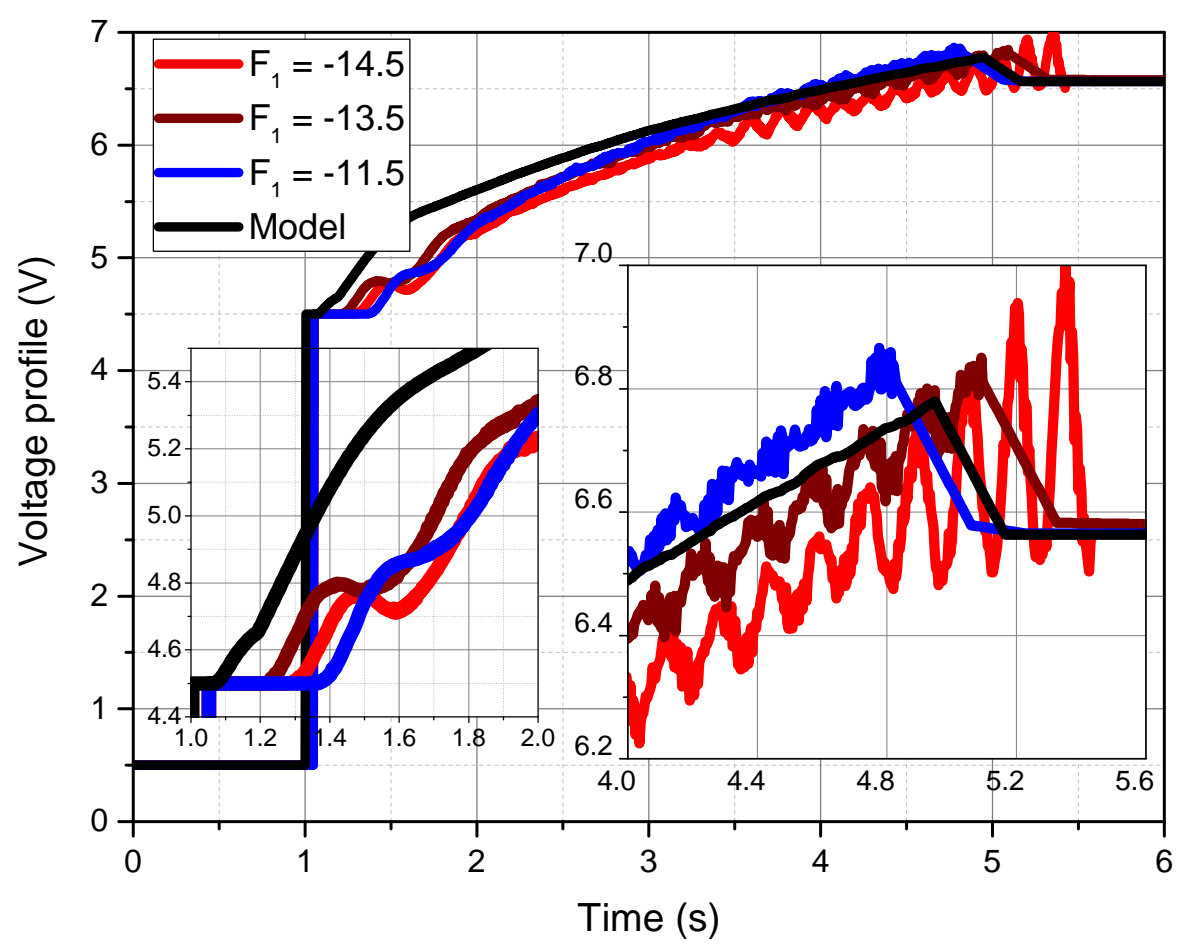

Figure 7.5: Experimental controller and modelled voltage profiles during the feed forward controller tuning at 35 bar inline pressure parameter.

is quadratic, the parameter $F_{1}$ has the biggest influence on the output of the controller. The acquired experimental controller voltage outputs are presented in Fig. 7.5 .

Sensitivity analysis could not be performed using standard Simulink Control optimisation toolboxes since the system comprises of Simscape components. Hence the analysis was performed by manually changing the controller parameters (feedforward, PI and so on) and checking the system's dynamics. Even though it was possible to tune the PID controller in the model, it was not feasible and safe to do in the real prototype. This is the main reason why the feedforward controller was chosen for it.

In this case, the simulated profile was acquired at $F_{1}=-12.5$ to show how different experimental voltage profiles correspond to the reference. The left inset shows the first $1 \mathrm{~s}$ after the valve opening, and according to the mathematical model of the valve, this stage is the most non-linear. It can be observed that the model performs faster than the original valve, reaching $5.4 \mathrm{~V}$ within $0.6 \mathrm{~s}$ in comparison to experimental 4.8-4.9 V. However, within the time of the period of 3-5 s, the simulated voltage profile and the prototype is almost the same. This supports the statement that the steady states of the valve in the hardware 


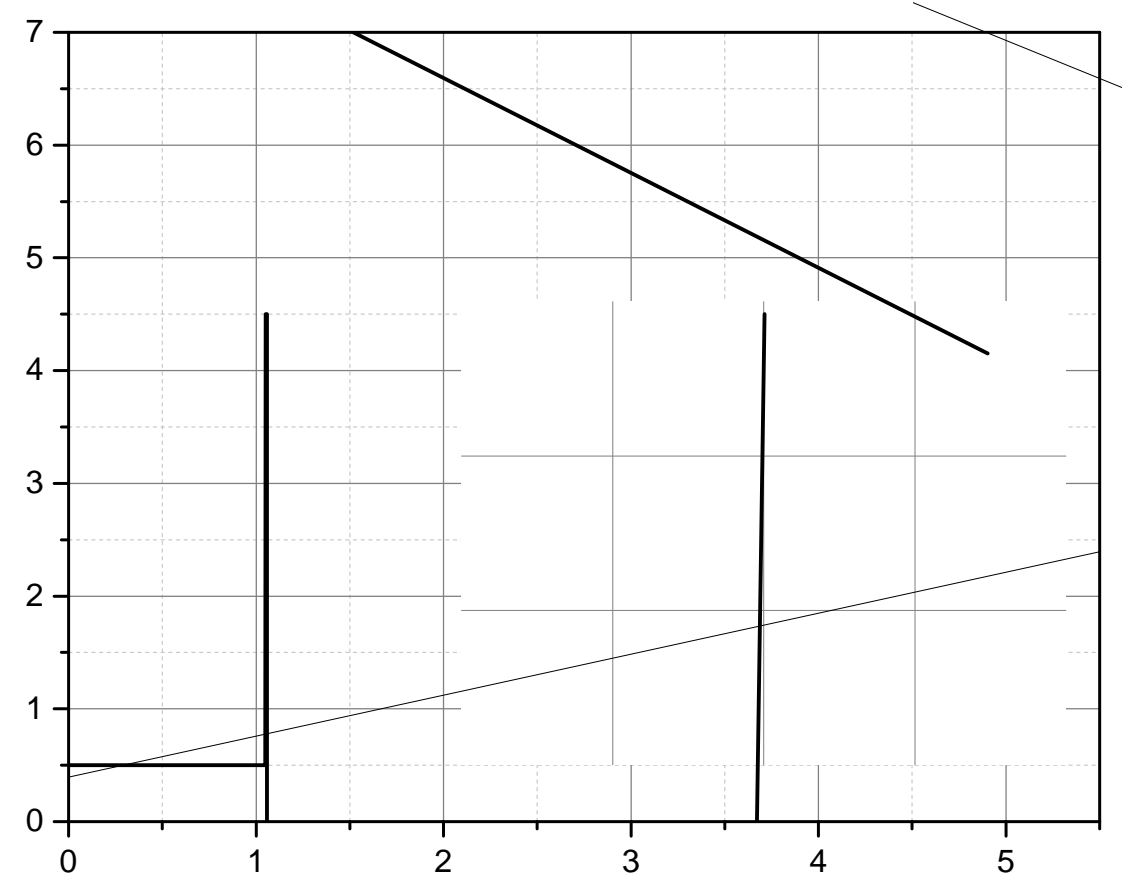

Figure 7.6: Simulated voltage profiles of the control valve with various feed forward controller parameters at 40 bar inline pressure parameter.

and the model are the same. A difference in the controller performance can be easily observed in details in the right inset (Fig. 7.5).

The larger the coefficient $F_{1}$, the more unstable the system becomes. It also lowers the voltage output profile. This instability can be compensated with the tuning of other coefficients of the controller (primarily the PI-controller). However, for the sake of understanding the controller operation, it is imperative to verify the behaviour of the system with only one parameter being changed. It is also possible to compare the voltage profiles at higher acceleration with the range of the $F_{1}$ coefficient from -10.5 to -15.5 , as it is presented in Fig. 7.6. It helps to identify how the controller coefficients influence the voltage output in the prototype.

Overall, the oscillations in the voltage profile do not necessarily lead to presence of oscillations in the velocity or phase signals of the generator. This is due to the hydraulic fluid inertia in the circuit and the combined inertia of the electro-hydraulic drivetrain. Therefore, sudden changes in the valve voltage will not always affect the dynamics of the motor and the generator, as was found during the experimental testing. This behaviour is shown in Fig. 7.7.

It shows a difference in the velocity profiles during the start-up of the sys- 


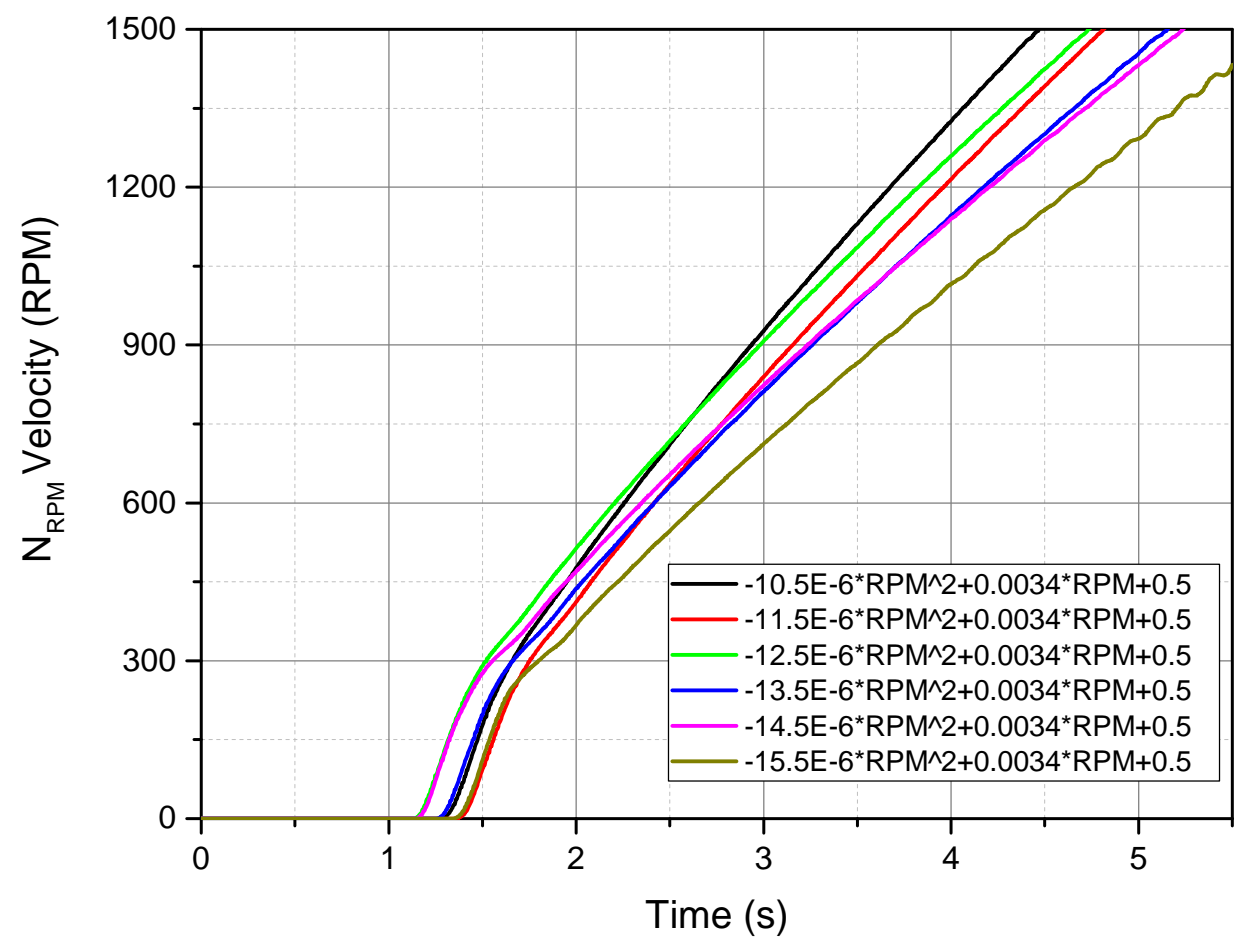

Figure 7.7: Simulated velocity profiles of the generator with various feed forward controller parameters.

tem, and also how these signals are different during the generator's acceleration. Moreover, the signal acquired with $F_{1}=-14.5$ depicts how insignificant the velocity transients in comparison to the significant voltage oscillations from Fig. 7.6.

The final step in the controller parameters verification is the phase difference correlation during the synchronisation process, which is depicted in Fig. 7.8. It shows slower acceleration of the generator, followed by failed synchronisation (for most values of $F_{1}$ ). The plot shows that the value of 11.5 for $F_{1}$ was the best option, which led to the system's synchronisation. It is also possible that another set of controller parameters will lead to another synchronisation moment. It should be noted that the phase difference plots are not fully presented, due to oscillations that were presented in the hardware system during this testing. This can be explained by complex dynamics and processes happening in the system (not measured and not accounted for in the model) and their influence on the controller. 

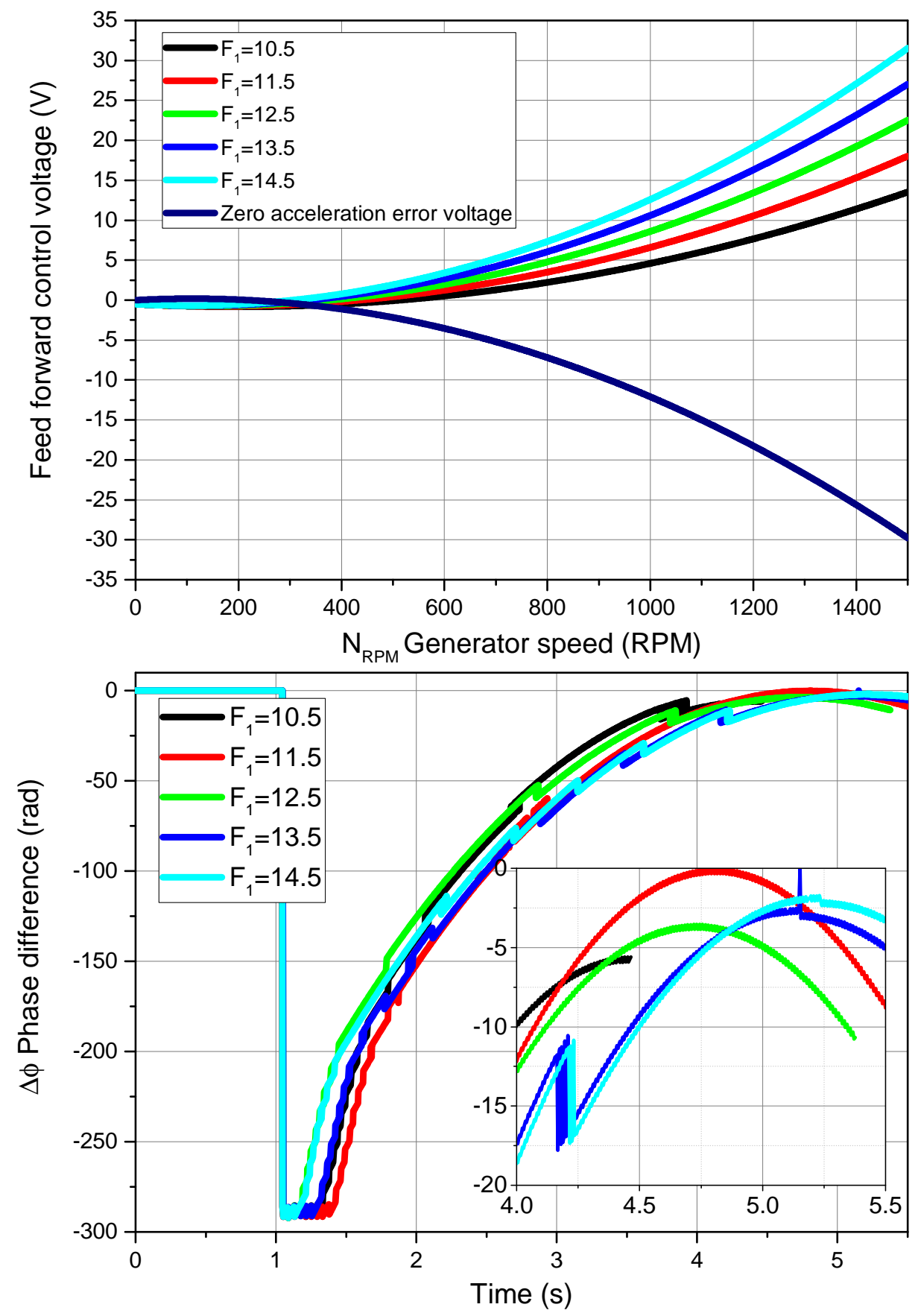

Figure 7.8: Simulated tuning of the controller. Top: influence of the feed forward parameters on the control voltage output; Bottom: Phase difference at different feed forward coefficients. 


\subsection{Demonstrated slow synchronisation scenario}

Example, experimental data from a successful synchronisation run, is shown in Fig. 7.9. The system was triggered at $1 \mathrm{~s}$, and a ballistic synchronisation trajectory was followed which led to synchronisation at $5 \mathrm{~s}$. After that time the generator's phase and frequency match, and follow the target grid cycle meeting all criteria required to allow the physical connection to the power grid.

A comparison between the Simulink model and our experimental hardware is provided by examining the control voltage output to the NG16 valve (Fig. 7.9(c)). This shows that during most of the acceleration period (e.g. $>2$ s) the experimental and simulated values closely agree, however small but significant differences can be observed within the first second after the system is triggered. This is due to the different dynamic behaviour of the experimental and simulated valves (remembering Fig. 5.12). This explanation is confirmed by Fig. 7.9 (d) which shows that the measured initial spike in acceleration in the real system is greater than the value in the equivalent simulation, due to the non-ideal transient behaviour of the valve during the rapid poppet motion which occurs at start-up. However, the PI-controller compensates for this deviation from simulated behaviour so that the experimental and simulated acceleration values converge to the same value for the period $2-5 \mathrm{~s}$. In both experiment and simulation, the controller switches from ballistic mode to gridfollowing mode at $5 \mathrm{~s}$, as shown by a sharp peak in the control voltage in Fig. 7.9 (c). This denotes that synchronisation has been achieved in both cases. In fact, the experimental system synchronises slightly later than the simulation (by one grid cycle, $20 \mathrm{~ms}$ ), which is due to the necessity to skip an extra grid cycle to correct the non-ideal behaviour followed during the first moments after the valve is opened.

A further set of experimental runs showed that the system is capable of synchronising within 4-5 s after being triggered. Even though the maximum allowable acceleration was limited to and the same during the runs, a difference in the drivetrain's dynamics resulted in slightly different synchronisation time. However, the control system was capable of reaching a synchronisation moment every time. Overall, the experimental results presented here prove the synchronisation concept in hardware, which was the most challenging objective of this research. The system was studied further including the comparison between the model and the hardware under different conditions.

A further set of experimental runs showed that the system is capable of synchronising within 4-5 s after being triggered. Even though the maximum allowable acceleration was limited to and the same during the runs, a differ- 

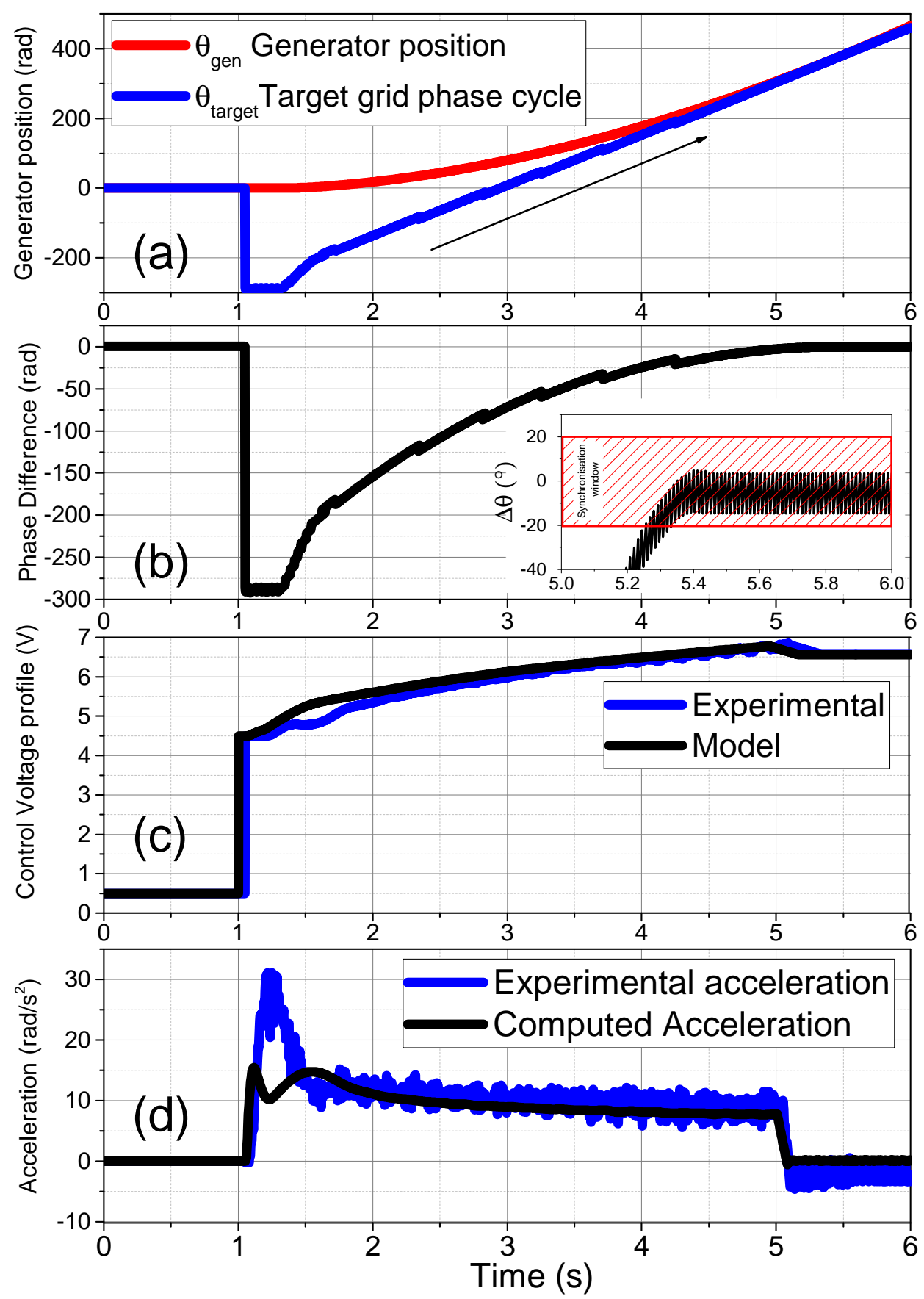

Figure 7.9: Experimental data from hardware verification of our ballistic synchronisation approach. plots show key parameters plotted against time (system is triggered at $t=1 \mathrm{~s}$ ). From top to bottom: (a) Phase angle of generator, $\theta_{\text {gen }}$, and target grid phase $\theta_{\text {target }} ;(\mathrm{b})$ Phase difference between generator and grid $\Delta \theta=\theta_{\text {gen }}-\theta_{\text {target }}$, within the synchronisation boundaries of $\pm 20^{\circ}$; (c) Control voltage fed to the proportional throttle valve; (d) Acceleration of the generator rotor (experimental and computed values). 


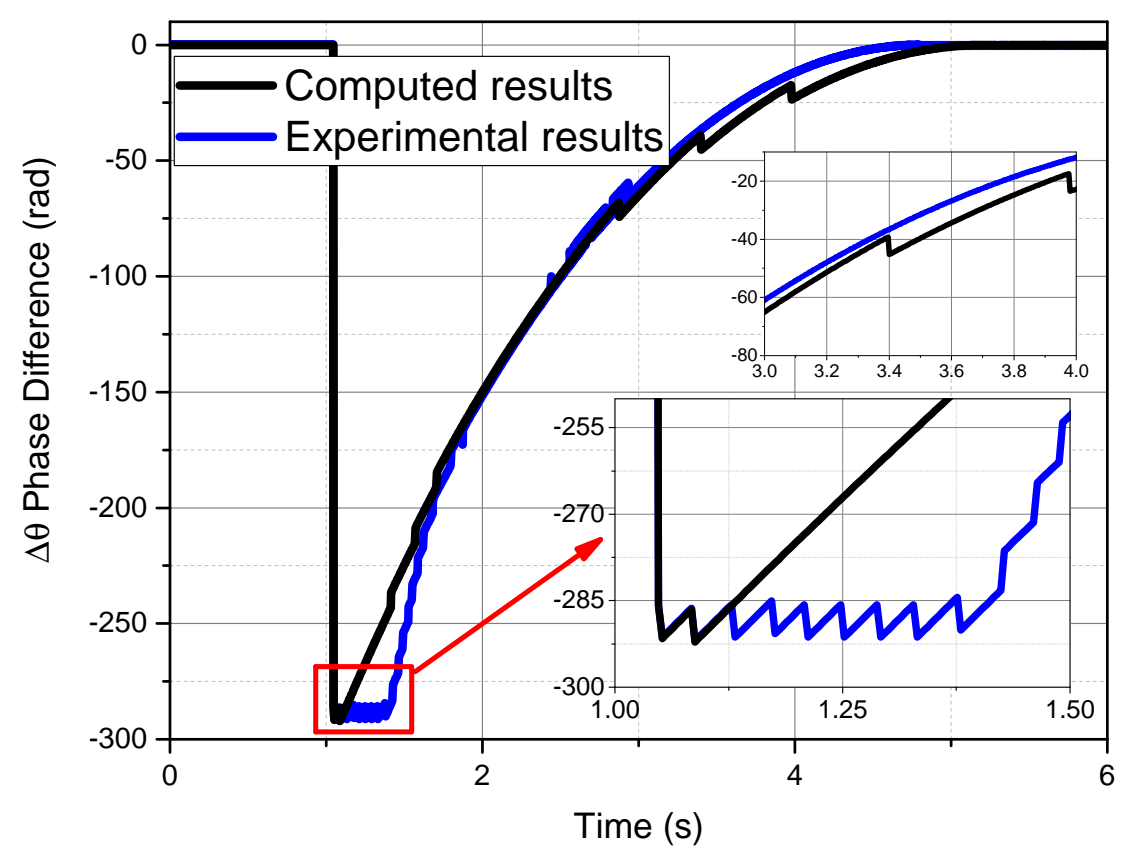

Figure 7.10: Phase difference between the generator and the grid - model and experiment.

ence in the drivetrain's dynamics resulted in slightly different synchronisation time. However, the control system was capable of reaching a synchronisation moment every time. Overall, the experimental results presented here prove the synchronisation concept in hardware, which was the most challenging objective of this research. The system was studied further including the comparison between the model and the hardware under different conditions.

\subsubsection{Comparison of the experimental and simulated results}

Even though the comparison between the mathematical model and the experimental results was partially presented in the discussion of the experimental results, it is imperative to discuss another system output, i.e., the phase difference during the synchronisation process. For instance, the phase difference plot (Fig. 7.10) depicts that the main difference between the model and the hardware is the number of the skipped grid cycles (lower inset).

It should be reminded that the phase difference is:

$$
\Delta \theta=\theta_{\text {gen }}-\theta_{\text {target }}
$$

Where the grid phase $\theta_{\text {grid }}$ is the same in the model and the hardware (as a generated frequency signal). Hence, the phase difference can be explained 


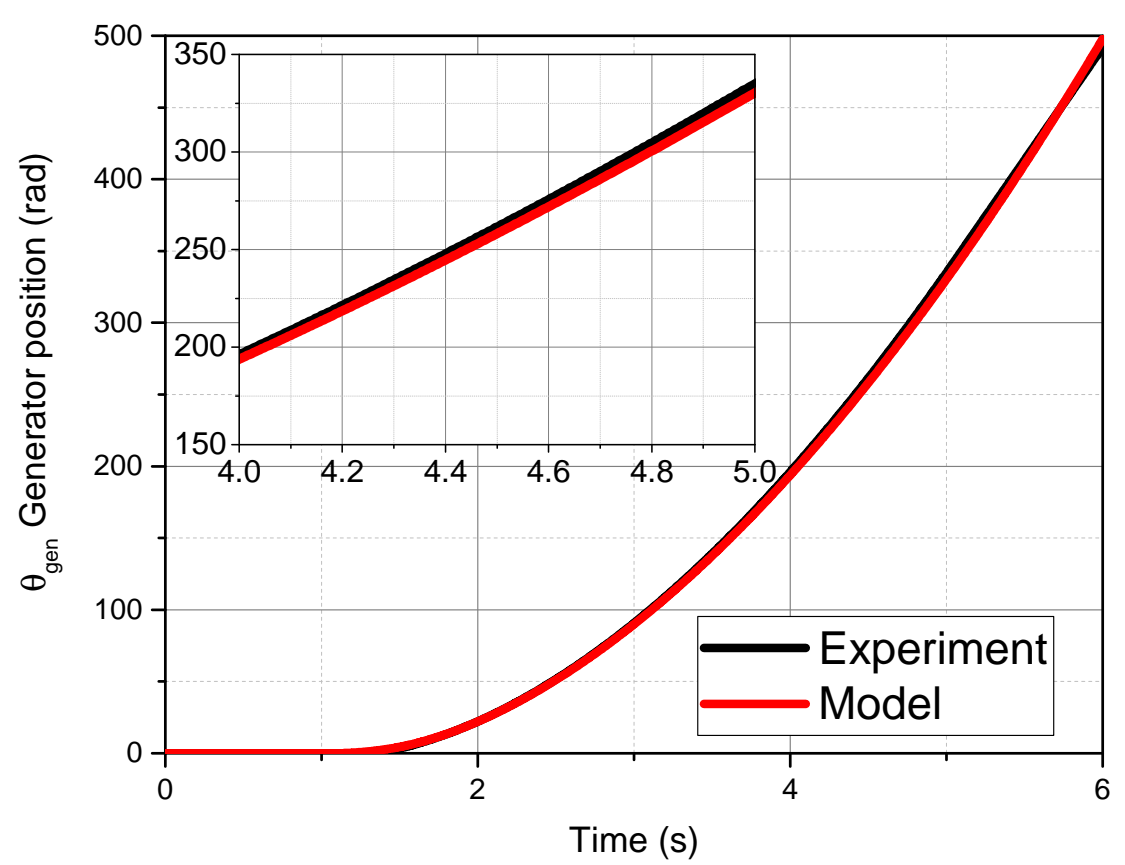

Figure 7.11: Comparison of the generator and the grid position - model and experiment.

only by the difference between the model and the real system concerning the generator phase.

The generator phase profiles can be compared to establish a difference between the simulated results and the prototype as it is presented in Fig. 7.11. It can be seen that the plots are close to each other, depicting that the dynamics of the motor in the model are simulated quite accurately.

Taking into account that the phase profiles are the same, the main reason behind the different number of the skipped grid cycles should be investigated further. Hence, the synchronisation acceleration equation should be analysed:

$$
\alpha_{\text {optml }}=\frac{1}{2} \frac{\omega_{\text {gen }}^{2}}{\theta_{\text {gen }}-\left(\theta_{\text {grid }}-2 n \pi\right)}
$$

where $\alpha_{\text {optml }}$ is the acceleration of the drive train $\left(\mathrm{rad} / \mathrm{s}^{2}\right), \omega_{\text {gen }}$ is the velocity of the drive train (rad/s), $\theta_{\text {gen }}$ is the generator phase (position) (rad) and $\omega_{\text {gen }}$ is the angular frequency of the generator ( $\mathrm{rad} / \mathrm{s}), 2 \pi n$ is a grid cycle ( $\mathrm{n}$ is an integer).

Since the grid phases are the same (synthetic grid phase signal $\theta_{\text {grid }}$ ) and the generator phase $\left(\theta_{\text {gen }}\right)$ is almost the same, the only parameter that can cause a difference in the number of the grid cycles is the velocity of the generator with the experimental and simulated comparison shown in Fig. 7.12. 


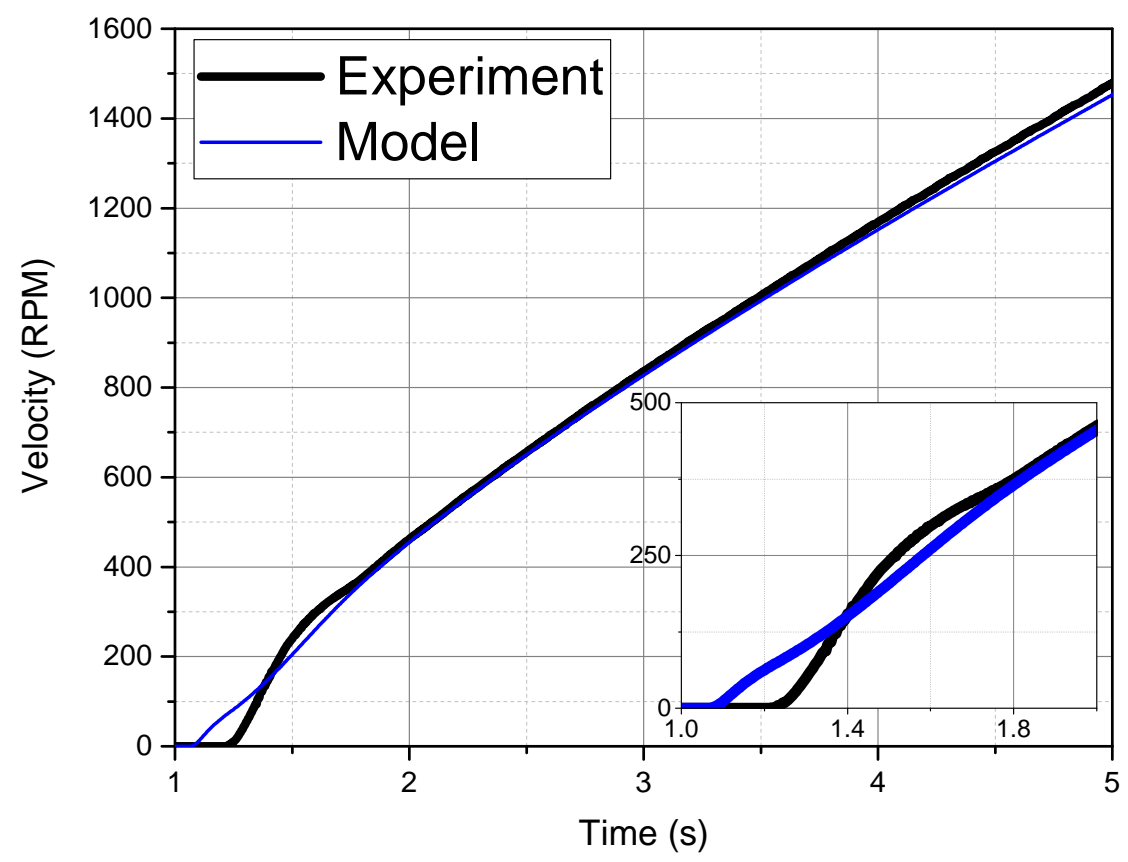

Figure 7.12: Comparison of the generator velocity - model and experiment.

From the plot, it can be seen that the main difference in the velocity profiles is observed during the generator start-up. It can be mainly explained by the difference in the valve's dynamics and also the difference in the controller output that tries to find the synchronisation moment. Hence, the most accurate comparison of the velocity of the generator and its model can be made with the use of a step voltage signal, as presented in Fig. 6.7.

The similarity of the velocity profiles proves that the inertia and the displacement of the electro-hydraulic drivetrain were measured and derived correctly. Hence, the difference in Fig. 7.12 can be explained by the behaviour of the control system due to the changeable temperature of the hydraulic fluid, hence the drivetrain's dynamics.

In summary, the behaviour of the generator is quite similar in the model and the prototype. There are a few fundamental differences between them, for instance, the influence of the temperature and viscosity on the acceleration of the motor. Even the main proportional throttle valve (TDA016) is prone to high non-linearity during its opening. All the processes that happened in the prototype were acquired during the experimental testing, but not necessarily that the system will be subject to them during the next run. A highly complex hydraulic system inherently introduces uncertainties into the system. From this perspective, it is required to conduct further research on the system's stability and the boundaries within which the controller and the system can operate 


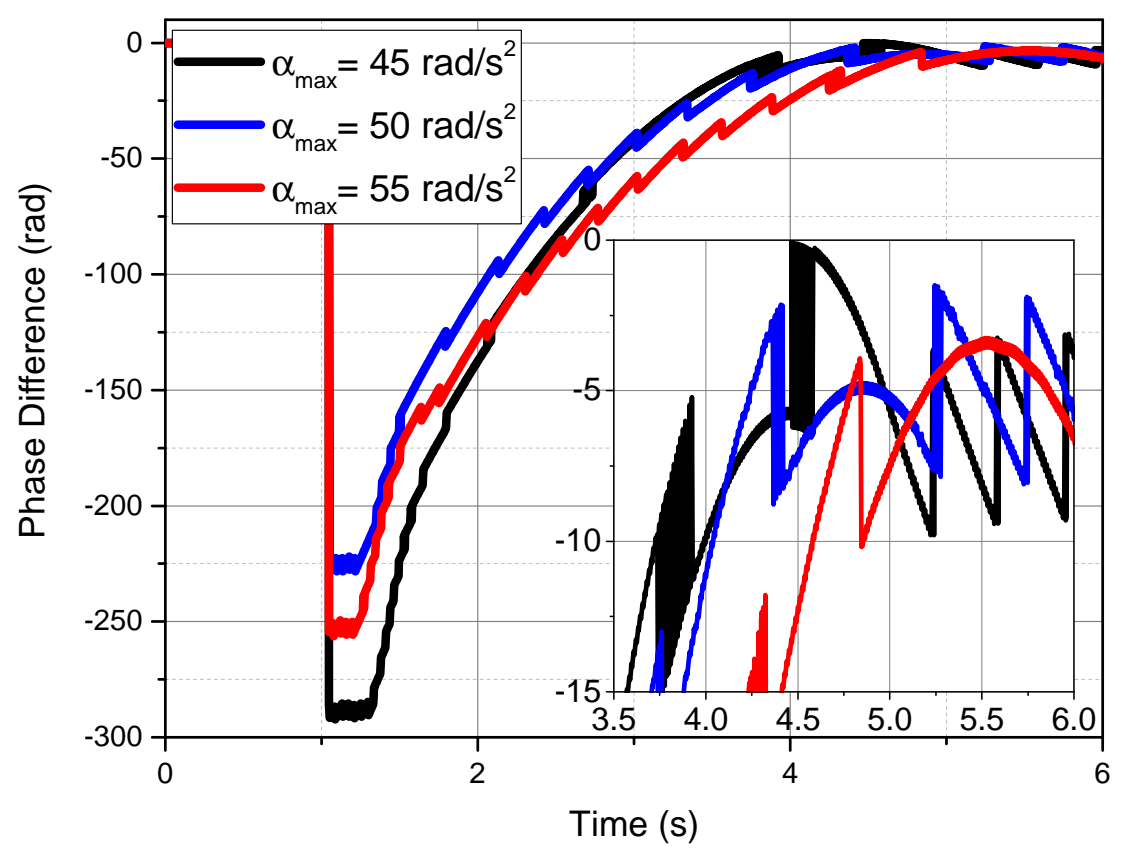

Figure 7.13: Performance of the system at different maximum acceleration values.

effectively (or at least can cope with).

\subsection{Modelled fast synchronisation scenario}

The experimental testing of the system was terminated after the successful verification of the main synchronisation control algorithm. The main reason why the testing was not performed at a faster acceleration is the instability of the central controller. The synchronisation algorithm was implemented in the cRIO - 9068 controller in the scan mode executing at a $1 \mathrm{~ms}$ sample rate. Due to the computational burden on the controller, jitters occurred around the zero phase difference moment. This behaviour is depicted in Fig. 7.13. The control system is employed to limit the maximum acceleration of the drive train. By changing this parameter it is possible to reduce or increase the synchronisation time of the generator. Overall, the faster allowable acceleration of the system, the faster synchronisation moment. However, in case of the presented system, the controller skips another grid cycle a moment before the zero phase difference is achieved. This is an indication that the controller cannot handle the computation meaning that the main loop executes slower than expected $(>1$ $\mathrm{ms})$.

In the case of the P-controller loop for the velocity and phase tracking these 
Figure 7.14: Prototype life cycle.

jitters could likely cause a delay in the operation of the code. This is because the valve's voltage decreases by the voltage decrement every iteration of the loop. If the loop executes slower that it was designed for, it will not slow down the generator to the reference synchronisation speed in a time efficient manner. Hence, it is likely that the generator will continue to accelerate to its maximum speed damaging the drivetrain. In case of the slow acceleration, it is possible to manually (by changing the control voltage in Labview) decelerate the generator, even if the P-controller loop fails to perform. Whereas at a fast acceleration, the same issue does not leave any time for the operator to stop the generator without using the emergency brakes manually. Based on the data collected from over 40 experimental runs, the decision was made not to perform the fast acceleration tests and instead use the mathematical model to verify this regime of operation as it is shown in Fig. 7.14.

These experimental runs showed that the system was able to achieve the required synchronisation windows, but the system was unstable. Almost $30 \%$ of the runs failed to reach synchronisation due to jitters within a synchronisation grid cycle.

Section 7.3 fully described and discussed the experimental results of the synchronisation testing, including the comparison between the model and the measured data. This proves the accuracy of the designed mathematical model, meaning that it can be employed for other related studies. This section focuses on the use of the mathematical model for the verification of the fast synchronisation scenario. Firstly, the initial mathematical model (based on the manufacturer data) was utilised for this study (in 2015 - 2016). According to the 
simulation results, the minimum synchronisation time was around $0.6-0.7 \mathrm{~s}$. However, with the use of the improved mathematical model, this time may be different.

To achieve the minimum synchronisation time, it is firstly required to change the acceleration algorithm, allowing the maximum generator acceleration. Secondly, the pressure source output pressure should be increased to 350 bar.

Based on Fig. 7.15 it can be seen that the synchronisation occurs at 0.85 - $0.9 \mathrm{~s}$ after the start-up of the system. It is slightly slower than the initially modelled 0.7 s synchronisation; however, this system still can be employed as a FIR ( $<6$ s.). Since the acceleration time also depends on the controller parameters, it is possible that with some sets of the parameters this time can be reduced. Taking into account the improved model it can be stated that with a suitable pressure source the real hardware can be accelerated almost as fast as the mathematical model depicts.

Initial dynamics of the drivetrain play a significant role during fast acceleration. In this case any difference between the model and the real hardware setup would result in tuning of the control system in order to reach stable and fast acceleration. As shown in Chapter 5, the real valve behaves differently from the hardware valve mainly in the first $20-25 \mathrm{~ms}$. In this case the control system would have to compensate this difference by changing the number of the skipped grid cycles, taking into account any differences in the drivetrain's dynamics. Meanwhile, the corresponding quasi states are the same, meaning that the behaviour of the system after the initial start is predictable by using the mathematical model. If the system does not synchronise within the modelled time frames, the mathematical model should be improved. It is mainly done by implementing hydraulic components (new orifce areas, bends and elbows, and so on) and by researhing a correlation between the dynamics of the train and the temperature of the hydraulic fluid.

This section verified that the proposed system could be potentially used as a FIR system when it comes to its response time. However, another requirement should be investigated, i.e., how the system will behave during contingent events in the power grid.

\subsubsection{Operation of the prototype at different frequency droop rates}

Every under-frequency event is followed by a frequency sag in the power grid. To verify the system's feasibility under these conditions, it is necessary to check the system response. According to some reports provided by the NZ System 

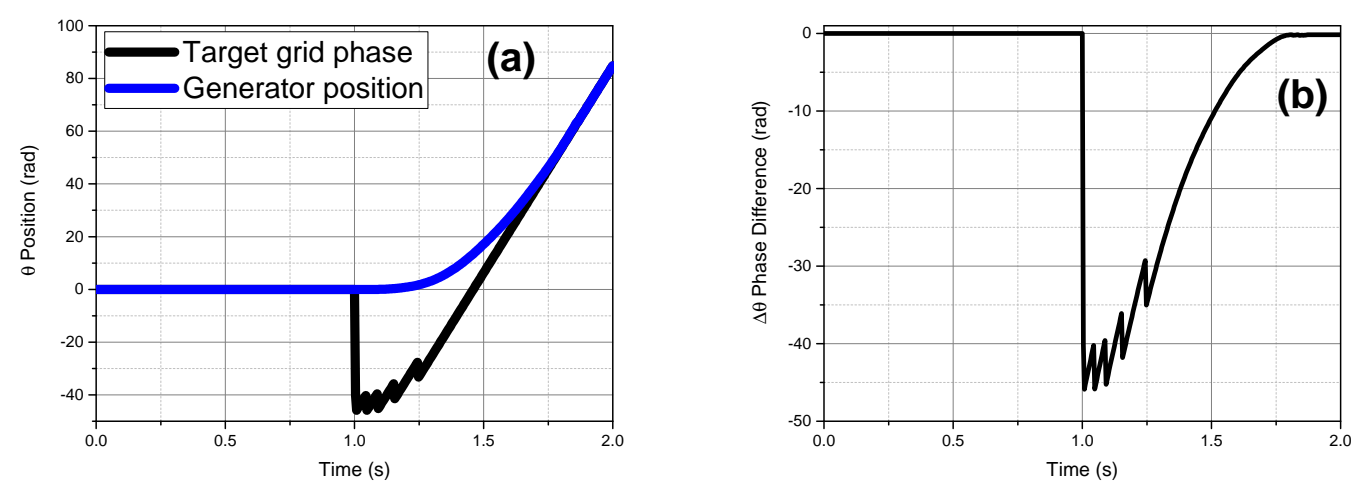

Figure 7.15: Simulated improved ballistic synchronisation approach during fast acceleration at 350 bar inline pressure and maximum acceleration. (a) Phase trajectories of the generator and the grid during acceleration. (b) Phase difference between the generator and the grid indicating successful synchronisation.
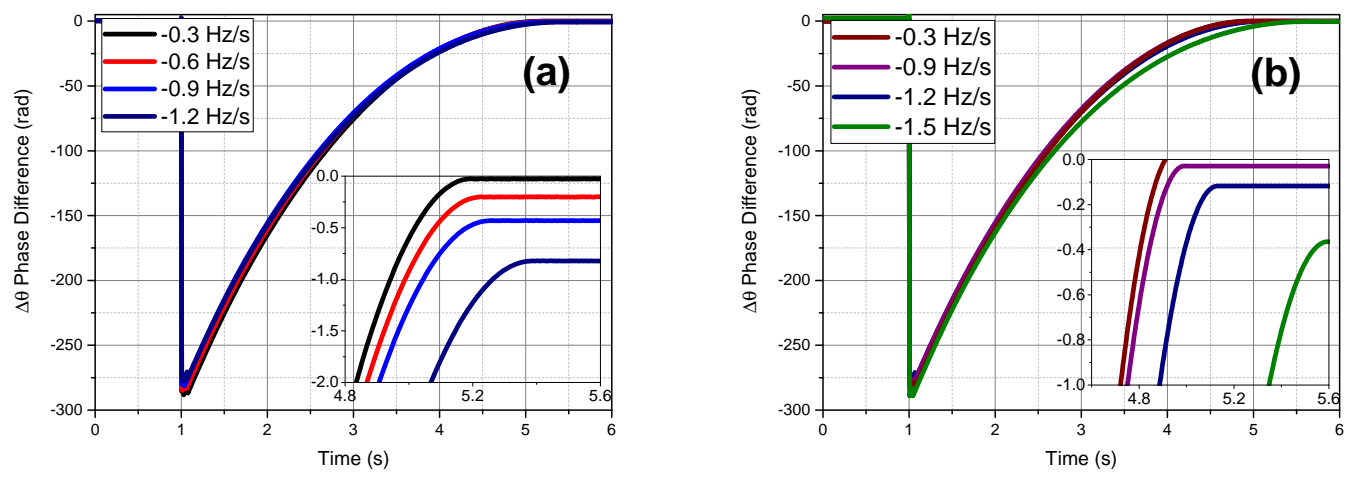

Figure 7.16: Simulated phase difference between the generator and the grid at different frequency droop rates with $F_{1}=14 \cdot 10^{-6}(\mathrm{a})$ and retuned $F_{1}=16 \cdot 10^{-6}$ (b).

Operator Transpower, the largest RoCoF recorded in New Zealand was 1.2 $\mathrm{Hz} / \mathrm{s}$, recorded in 2011. This value can be used for the verification of the acceleration approach and the system's stability as the potential worst case scenario. To cover a variety of frequency sags (hence under frequency events), the system was simulated at $0.3 / 0.6 / 0.9 / 1.2 \mathrm{~Hz} / \mathrm{s}$ as is shown in Fig.7.16 (a).

The phase difference requirement for synchronisation according to the IEEE 1547 standard [121] is $20^{\circ}\left(15^{\circ}\right.$ recommended according to the manufacturer $\mathrm{ABB})$. It can be seen that at the frequency droop rates higher than $0.6 \mathrm{~Hz} / \mathrm{s}$, the phase difference between the grid and the generator reaches almost 0.5 $\mathrm{rad}$. To ensure that the system can operate under severe frequency droops it is required to tune the controller parameters. It should help to achieve the phase 
difference of $0.26 \mathrm{rad}$ maximum at the frequency droop rates up to $-1.2 \mathrm{~Hz} / \mathrm{s}$. As an example of this tuning, the following plot Fig. 7.16 (b) can be depicted.

Feed forward parameters can change a voltage output of the controller at a certain generator's speed. This affects the acceleration and position of the rotor during a synchronisation process. Meaning that different feed forward parameters contribute towards different phase differences at the moment of synchronisation.

The difference between both of these plots is easily noticeable and relates to the phase difference at the frequency droop rate of $-1.2 \mathrm{~Hz} / \mathrm{s}$. At this sag, the phase difference achieves $0.11 \mathrm{rad}$, which is significantly lower than the phase requirement of $0.26 \mathrm{rad}$. Moreover, it means that the controller is capable of generating the required control voltage to the valve at the frequency droop rates higher than $-1.2 \mathrm{~Hz} / \mathrm{s}$, meaning that the system can operate during severe contingent events.

It should be noted that there are a few controller parameters that can be tuned to achieve the required phase difference. However, regarding the feedforward controller, these changes can be quickly and mathematically implemented, taking into account the frequency droop rate and the corresponding value of $F_{1}$. With the use of these two parameters, it is possible to find a mathematical relationship that helps to achieve a zero phase difference regardless of the frequency droop rate and hard-wired controller parameters.

This section summarises the research on the feasibility analysis of the proposed system as a FIR system. It was proved that the system could be synchronised in the time efficient manner (within $1-6 \mathrm{~s}$ ) and also cope with severe frequency droop rates up to $(-1.2 \mathrm{~Hz} / \mathrm{s})$ and even step frequency sags. Further research will focus on the robustness of the system and its stability during malfunctions in the system.

\subsubsection{Robustness of the designed control system}

Apart from the unstable and polluted frequency from the grid, the prototype also can introduce some instabilities into the system's operation:

- The inline pressure sensor that measures the transmitted pressure from the accumulator to the electro-hydraulic drivetrain. Regarding the safety of the operation this malfunction does not cause any serious issues; however, will affect the operation of the swash plate drive mechanism. The prototype was designed in the way that it can run up to 15 minutes depending on the amount of the stored gas in the bottles. When the accumulator pressure drops (as well as the flow rate), the displacement of the 
motor should be decreased in order to be able to run at the synchronisation speed (grid frequency). In case of the faulty inline pressure sensor, the swash plate displacement compensation algorithm will not operate; hence the synchronisation speed will not be achieved.

- Pressure sensors across the valve and the motor are important for the initial verification of these devices, but not crucial when it comes to the normal operation of the prototype.

- Flowmeter was primarily used for the verification of the main hydraulic components in the drivetrain. This measuring device can mainly be used for the swash plate mechanism. Any possible malfunctions of this mechanism will cause the same issues as the faulty inline pressure sensor for displacement regulation.

- Temperature sensors in the system are important to avoid the valve and the motor overheating. It is a significant factor during the experimental testing of the system, but their use during normal operation of the system is not required. Hence, the faulty sensors and readings will not cause any serious disruptions to the prototype operation.

- The only electronic component that was specifically designed for this prototype is the amplifier that inputs the valve opening voltage signals from the controller. It is needed to amplify the current from the controller $(20 \mathrm{~mA})$ to the maximum of $1.05 \mathrm{~A}$, which is the full opening current for the NG16 valves. Since this device controls the opening of the control valve NG16 and also other valves in the hydraulic circuit, its stable operation is required for the system stability and performance. Any malfunctions of the amplifier will cause unstable behaviour from the motor and the generator that can potentially cause damage. The only solution to this issue is the use of the installed safety relief relay. If the amplifier starts to send its maximum opening voltage (meaning the maximum motor's acceleration), the installed encoder can trigger a safety mechanism that will turn off the amplifier's power input.

- The last safety mechanism is the hydraulic drum brake that is fitted on the coupling between the motor's shaft and the generator's shaft. The brake is controlled from another controller (TRIO Motion) to ensure its zero fault operation, even in case of the unstable NI cRIO controller behaviour. This brake was designed specifically for this prototype and is capable of stopping the electro-hydraulic drivetrain at its maximum acceleration and torque. 
To complete the stability studies, it is necessary to test the mathematical model of the prototype under different conditions, representing different mechanical and / or electrical failures in the hardware built.

\subsubsection{Stability analysis of the system employing the mathe- matical model}

The studied prototype is subject to a large number of different non-linearities and disturbances. Some of them have mechanical nature (hydraulic fluid viscosity, stickiness of the valve's poppet, worn bearings), whereas some are related to the electrical field (inaccurate controller/module, the different inductance of the circuit's elements). As was mentioned above, the system was not stable all the time during the experimental testing. Therefore, the safest and the most feasible way to verify the system's stability boundaries is to use its realistic mathematical model. For this study, the same mathematical model of the prototype was used, with some subtle modifications in order to generate an error signal affecting the controller's voltage output; hence, the dynamics of the system as it is depicted in Fig. 7.17 (a). The error signal is modelled as a step signal overlapping the valve voltage input. It's amplitude is taken as a percent from the ideal voltage signal. From the voltage profile presented it can be seen that the larger the disturbance, the slower voltage increases in the beginning (1-2 s). Even though the profile's final voltage value can be the highest at the largest disturbance. 


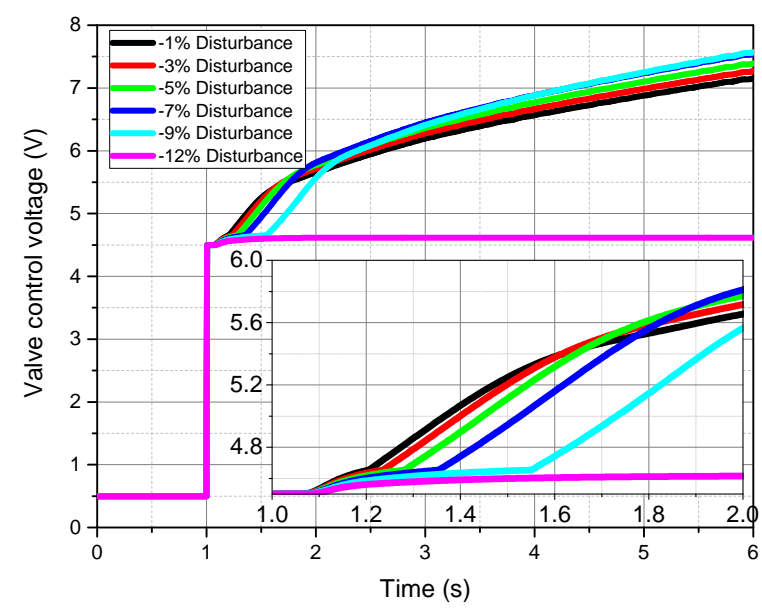

(a) Voltage profiles for the presented stability analysis.

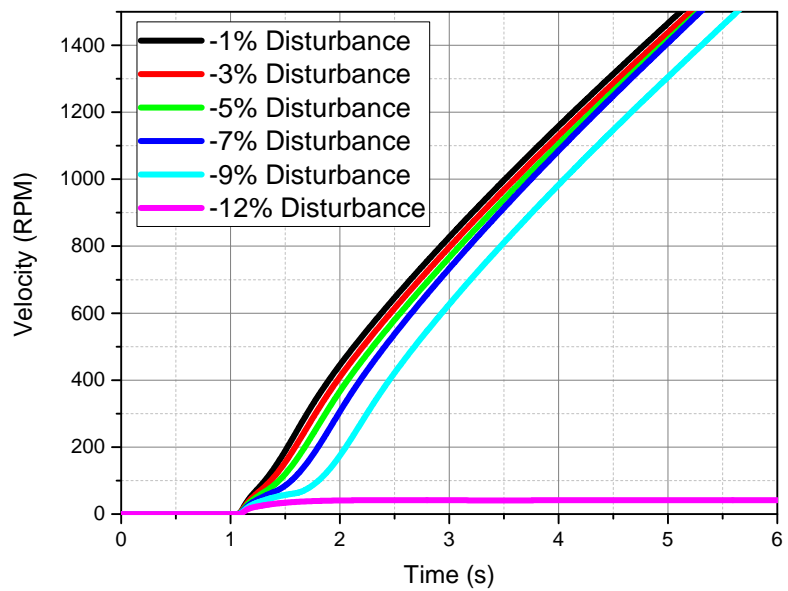

(b) Velocity profiles for the presented stability analysis.

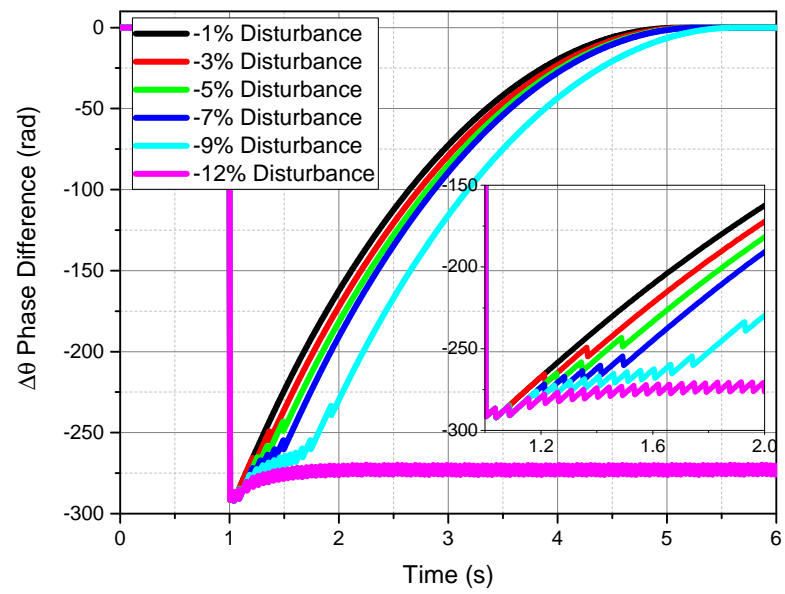

(c) Phase difference profiles for the presented stability analysis.

Figure 7.17: Stability analysis of the proposed control system employing the realistic mathematical model - malfunctioned TDA016. 


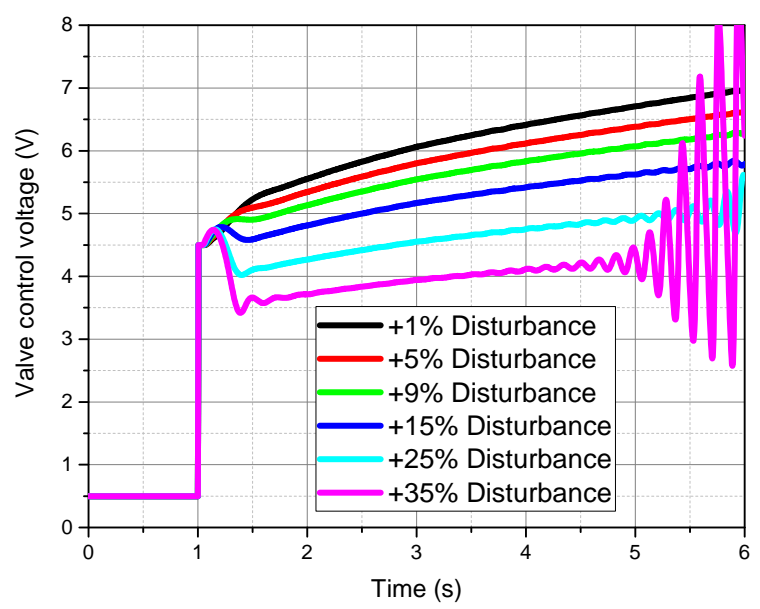

(a) Voltage profiles for the presented stability analysis.

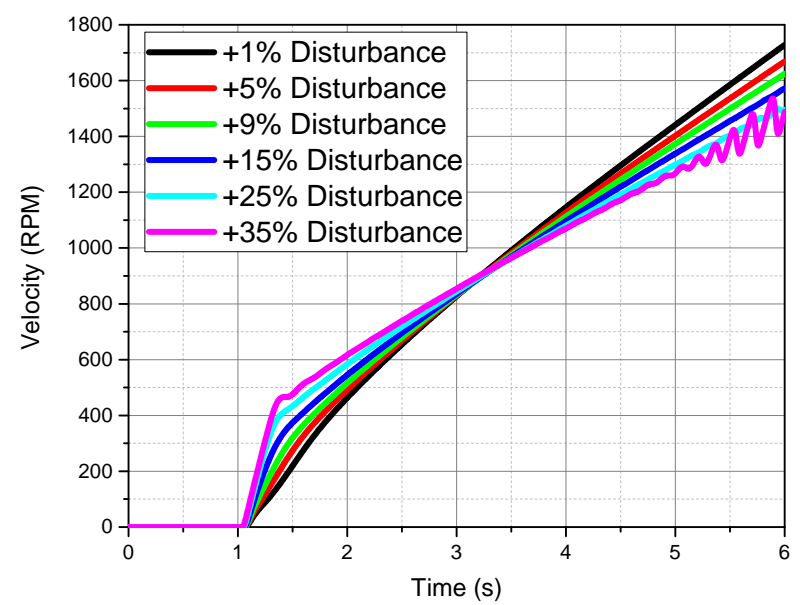

(b) Velocity profiles for the presented stability analysis.

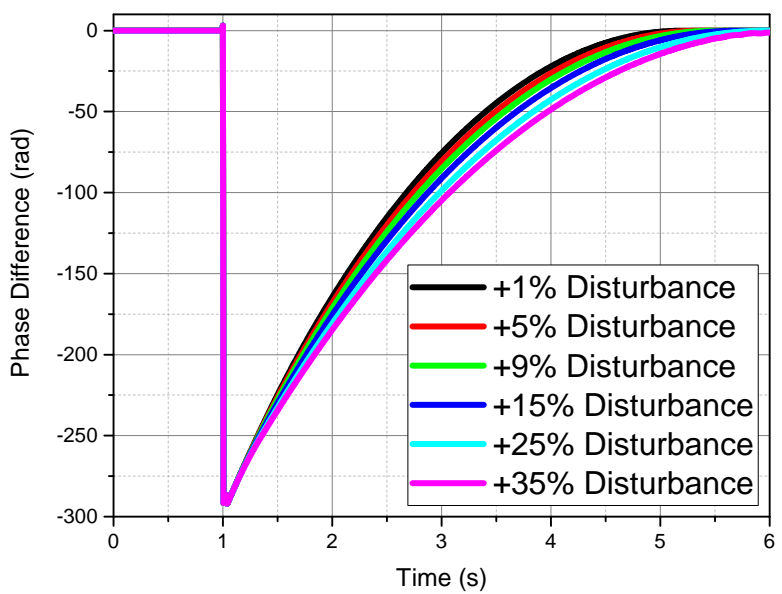

(c) Phase difference profiles for the presented stability analysis.

Figure 7.18: Stability analysis of the proposed control system employing the realistic mathematical model - malfunctioned TDA016. 
It can be observed that the control system cannot cope with the disturbances up to $-12 \%$. This kind of malfunction can indicate a serious fault in the control valve's poppet or a damaged bearing in the electro-hydraulic drivetrain. Dynamic dependencies between the voltage variations and the generator's velocity are depicted in Fig. 7.17 (b). It can be seen, that during the system's start-up the velocity profiles mimic the voltage behaviour, i.e. the larger the disturbance, the slower the generator accelerates.

To finalise the stability analysis of the presented control system, it is necessary to study the phase difference correlations during the generator's synchronisation - Fig. 7.17 (c). Even though the disturbances up to $0.9 \%$ change the acceleration profile and increase the synchronisation time, the controller is still able to perform correctly. The controller fails to perform at $12 \%$ disturbances.

The same stability analysis with a positive disturbance should be studied also, with the corresponding plots presented in Fig. 7.18. It can be seen from the phase difference plot (Fig. 7.18 (c)) that the control system can easily cope with disturbances up to 30-35\%. Both of the disturbance simulations show the worst-case scenario of the malfunctioned TDA016. In this case, it represents the poppet's malfunction if it is stuck in the position that does not directly correlate with the applied control voltage to the coil. Therefore, instead of the expected flow rate (hence the dynamics of the drivetrain), the control system experiences this unpredictable behaviour of the system and adjusts the control voltage to compensate these changes in the dynamics of the generator and the motor. This disturbance can be considered as constant since the poppet of the valve does not oscillate around its current position. Hence, the next step is to verify the behaviour of the system when it is subject to a changeable disturbance. This process represents a failed bearing or noise in the controller board and its modules ans presented in Fig. 7.19. 


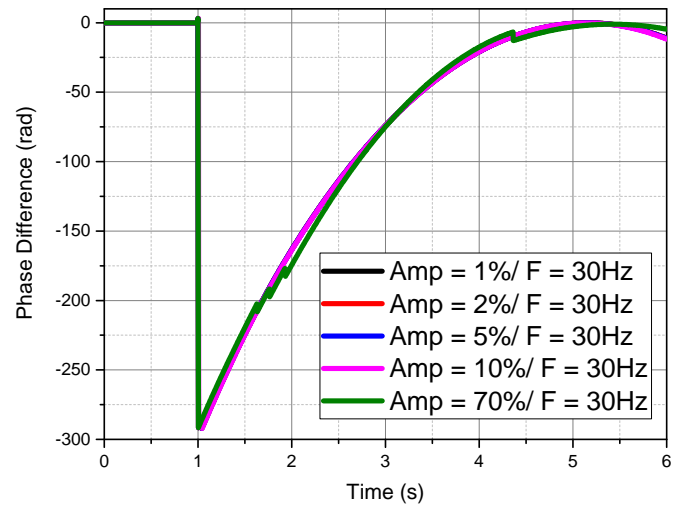

(a) Phase difference profiles at different error signal's amplitudes at 30Hz.

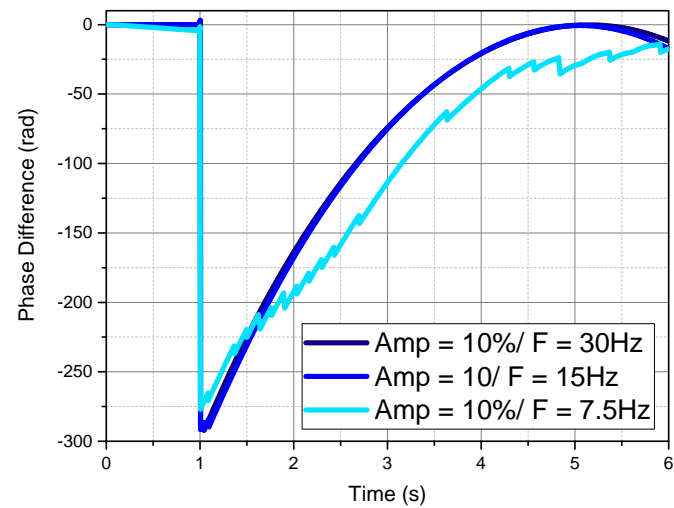

(b) Phase difference profiles at different frequencies of the error signal with the same amplitude.

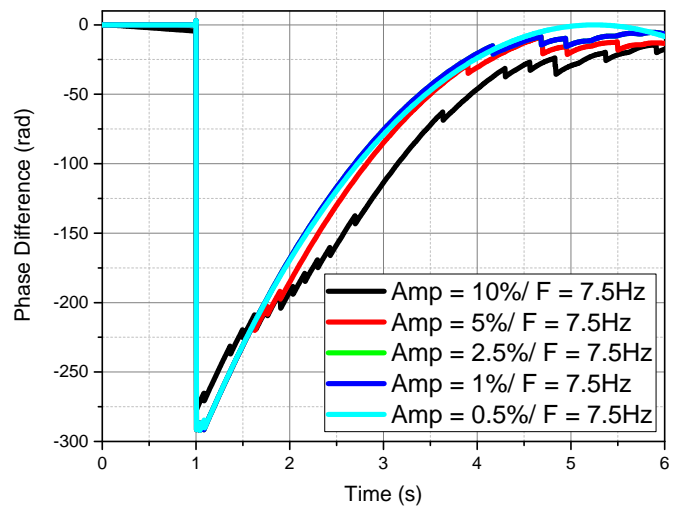

(c) Phase difference profiles at different amplitudes of the error signal at the same frequency of $7.5 \mathrm{~Hz}$.

Figure 7.19: Stability analysis of the proposed control system employing the realistic mathematical model - failed bearing simulation. 
The research starts with the testing of a noise signal at the frequency of $30 \mathrm{~Hz}$ and different amplitudes - Fig. 7.19 (a). This frequency was chosen to represent a possible malfunction of mechanical components in the hydraulic circuit. It can be observed that at the disturbance of $70 \%$ the control system fails to perform, coping with the lower levels of disturbances. It can be speculated that this is due to the robustness of the system; however, this is mainly due to the frequency of the noise. Since it is a sine wave signal that is overlapped with the control voltage signal, its average (mean) value is almost the same as its original voltage profile. Due to the overall inertia of the electrohydraulic drivetrain and the hydraulic inertia of the mineral oil, the voltage changes too fast to affect the drivetrain dynamics. Thus, the phase difference during synchronisation is almost unaffected, unless the amplitude of the disturbance achieves the values that can't be handled by the main controller in the system.

Assuming that $10 \%$ disturbance is the maximum reasonable level that can be observed in the system, the next step in the stability analysis would be a search for the frequency when this $10 \%$ affects the synchronisation mechanism. In this case, Fig. 7.19 (b) depicts that the frequency of $7.5 \mathrm{~Hz}$ was low enough to make the controller fail under the same level of disturbances. Lastly, it is possible to find the lowest level of disturbance that does not affect the controller in the prototype, as is summarised in Fig. 7.19 (c). At low frequencies, the tolerated level of disturbance is around $0.5 \%$, proving that the lower the frequency of the disturbances, the lower the amplitude of the disturbance that can be coped with by the controller.

\subsubsection{Influence of the temperature and viscosity of the fluid on the synchronisation process}

The dynamics of hydraulic systems are nonlinear and depend on a large number of factors. One of the biggest is the temperature (hence viscosity) of hydraulic fluid. In the case of the studied system, this influence was studied in [122, 123]. However, these studies focused on water-glycol blends, which was a technical solution that was proposed during the design of the prototype. Due to some cost restrictions imposed by the client (associated with the fluid change), the decision was made to continue to use the current hydraulic fluid - mineral oil UNIVIS 46. Oils are less prone to significant changes in viscosity in comparison to water-glycol solutions; however, it is still feasible to check this correlation. In order to calculate this correlation, it is necessary to derive the fluid's viscosity at different temperatures according to the procedure from 
[124].

The oil density can be derived as:

$$
\rho_{t P}=\rho_{15} \cdot K_{t} \cdot K_{p}
$$

where $\rho_{15}$ is the oil density at $15{ }^{\circ} \mathrm{C}$ and zero overpressure, $K_{t}$ is the temperature correction coefficient, which is calculated according to:

$$
K_{t}=e^{-\alpha_{15} \cdot(t-15)\left(1+0.8 \cdot \alpha_{15}(t-15)\right)}
$$

$K_{P}$ is the overpressure correction coefficient

$$
K_{P}=\frac{1}{1-\gamma_{t} P 10^{-3}}
$$

Coefficient of volumetric expansion at $15^{\circ} \mathrm{C}$ is calculated according to the formula:

$$
\alpha_{15}=\frac{K_{0}+K_{1} \rho_{15}}{\rho_{15}^{2}}
$$

where $K_{0}=613.97226$ and $K_{1}=0$. Overall the coefficient of volumetric expansion at given temperature can be derived as:

$$
\alpha_{t}=\alpha_{15}+1.6 \cdot \alpha_{15}^{2}(t-15)
$$

Compression coefficient is calculated according to the equation:

$$
\gamma_{t}=10^{-3} \cdot e^{-1.62080+0.00021592 \cdot t+\frac{0.87096 \cdot 10^{6}}{\rho_{15}^{2}}+\frac{4.2092 \cdot t \cdot 10^{3}}{\rho_{15}^{2}}}
$$

By summarising the derived densities and viscosities, they can be presented in Table. 7.3 ,

Based on the calculated values, it is possible to run the mathematical model to verify the dynamics of the electrohydraulic drivetrain as it is presented in Fig. 7.20 (a). Step response is the easiest way to verify a difference in the electro-hydraulic drivetrain's dynamics, without any influence from the control system. In the case of the generator's velocity, it can be seen that the dynamics of the drivetrain are different during its acceleration. It results in different maximum speed that can be achieved within the same period (6s in this case). 
Table 7.3: Physical properties of the hydraulic fluid under different temperature conditions

\begin{tabular}{llll}
\hline \hline $\mathrm{T}\left(\mathrm{C}^{\circ}\right)$ & $\mu$ (Pas) & $\rho\left(\mathrm{kg} / \mathrm{m}^{3}\right)$ & $\nu\left(\mathrm{mm}^{2} / \mathrm{s}\right)$ \\
\hline-20 & 7.613798368 & 907.1 & 8393.560101 \\
-10 & 1.929556775 & 900.3 & 2143.237559 \\
0 & 0.652433557 & 893.4 & 730.2815732 \\
10 & 0.271101731 & 886.5 & 305.8113155 \\
20 & 0.131200806 & 879.5 & 149.1765849 \\
30 & 0.071299429 & 872.5 & 81.71854369 \\
40 & 0.042404383 & 865.5 & 48.99408829 \\
50 & 0.027090518 & 858.5 & 31.55564156 \\
60 & 0.018335728 & 851.4 & 21.53597418 \\
70 & 0.013010687 & 844.3 & 15.41002895 \\
80 & 0.009600855 & 837.2 & 11.46781518 \\
90 & 0.007321033 & 830 & 8.820521255 \\
100 & 0.005739806 & 822.9 & 6.975095526 \\
\hline
\end{tabular}




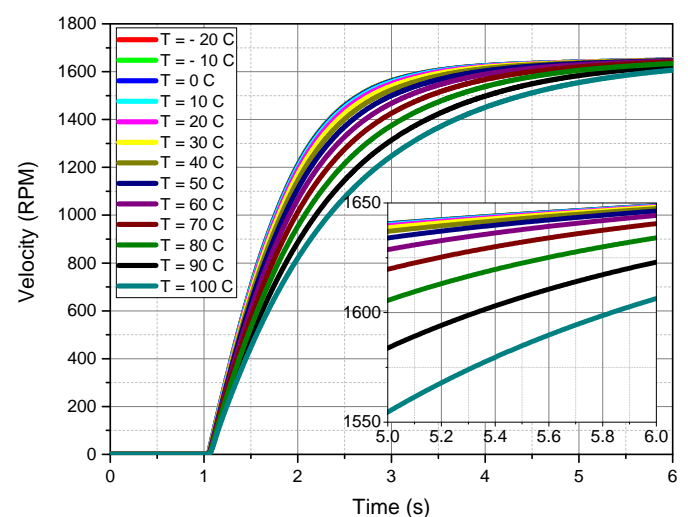

(a) Velocity profiles of the hydraulic motor at different hydraulic fluid temperatures.

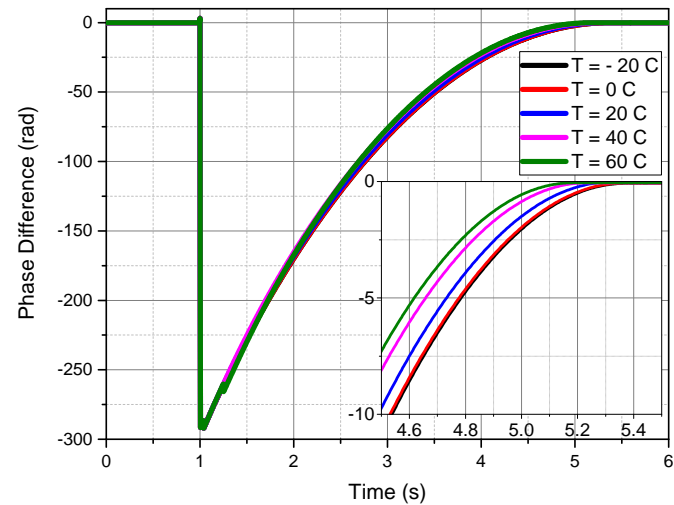

(b) Phase difference profiles of the hydraulic motor at different hydraulic fluid temperatures

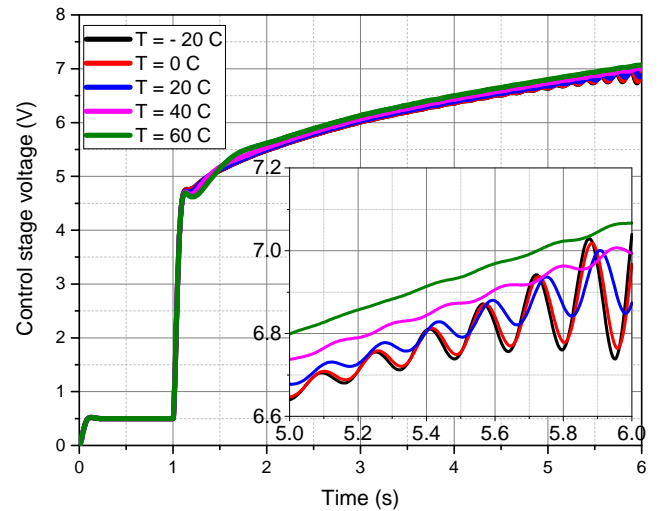

(c) Control voltage profiles of the hydraulic motor at different hydraulic fluid temperatures

Figure 7.20: Dynamics of the system at different temperatures and viscosities of the hydraulic fluid (UNIVIS 46 mineral oil). 


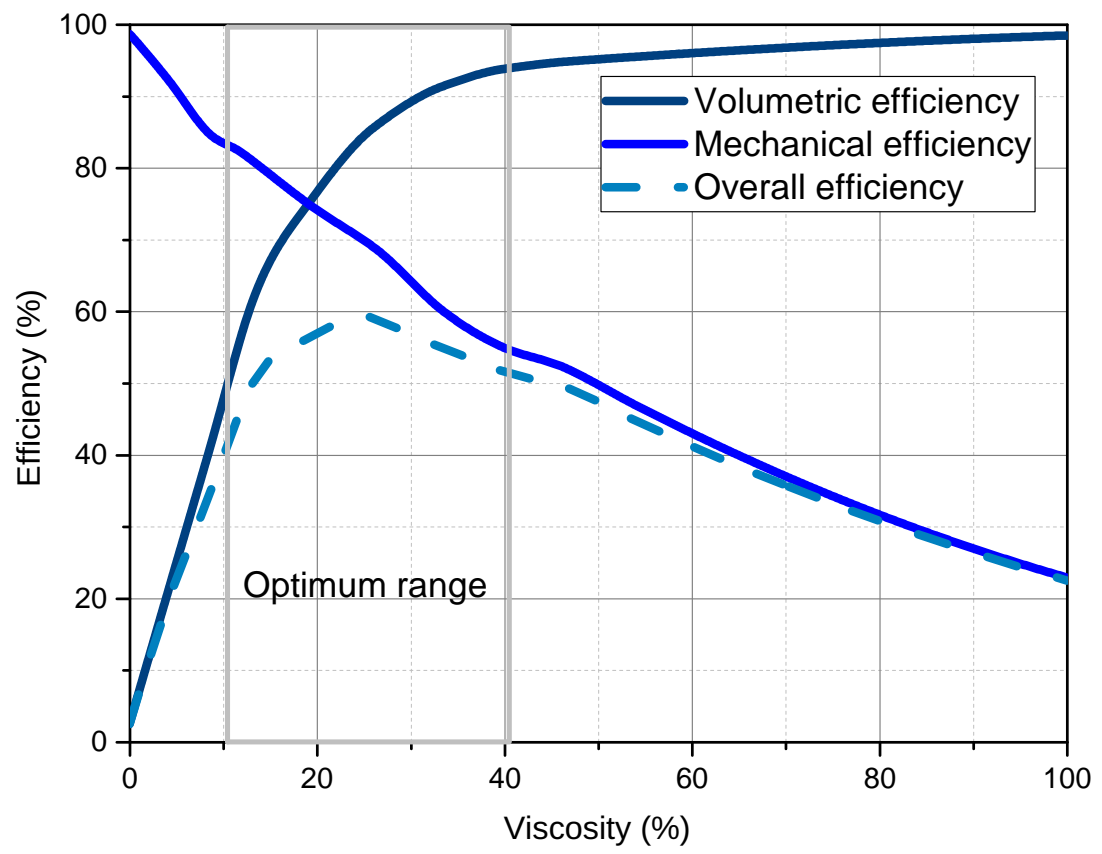

Figure 7.21: The optimum operating range of a hydraulic system depends heavily on fluid viscosity [125].

The lower the fluid density and viscosity, the lower maximum velocity can be achieved at the same voltage input. Which contradicts the common assumption that since it is easier for hydraulic fluid to flow through a motor, its velocity should be higher. However, in real life, it can be explained by a viscosity efficiency plot, like the one depicted in Fig. 7.21. A challenging question is the choice of hydraulic fluid since the oil viscosity affects the overall efficiency of a hydraulic system. With the lower viscosity of a fluid, its volumetric efficiency decreases and the hydraulic system becomes less efficient and responsive. On the other hand, high fluid viscosity results in low mechanical efficiency, which leads to friction, cavitation, and mechanical failures. Kinematic viscosity is also known as a "viscosity density"; therefore it determines a momentum that hydraulic fluid can transfer. Hence the higher the viscosity, the more momentum can be transferred; it also refers to the term "momentum diffusion". It results in the following plot that summarises both mechanical and volumetric efficiency in correlation to the fluid's viscosity [125].

The rule of thumb is that a hydraulic fluid should be chosen to ensure that within the operational temperature range, the overall efficiency of the system should be at its maximum. It should be noted that there are some chemical solutions and additives available on the market to address this challenge; however, in the scope of the project, we focus on pure mineral oil and water-glycol 
blends.

Regarding the synchronisation process, different viscosities mean different phase difference profiles (due to different dynamics) - Fig. 7.20 (b). Regardless of the temperature of the hydraulic fluid, the generation unit can still achieve synchronisation. The phase difference profiles are different before the synchronisation moment due to the different generator phase's profiles. The successful synchronisation means that the controller changes the output voltage to achieve synchronisation regardless of the viscosity of the fluid. This process can be observed in Fig. 7.20 (c). This results in different control voltage profiles being required to overcome changeable dynamics and reach a zero-phase difference between the generator and the grid.

This subsection highlights the influence of temperature on the dynamics of the system, assuming that this temperature is constant throughout the system's operation. However, in real life, the temperature of the hydraulic fluid increases while the system operates. Even though it is possible to model this process, for the dynamics comparison, it is more feasible to have a constant and stable temperature profile. The friction of the mechanical components, as well as the hydraulic power unit, produce heat increasing the fluid temperature. Due to the nature of the testing, when the control valve has been opened multiple times, the temperature profile increases drastically from the ambient temperature to $50-80{ }^{\circ} \mathrm{C}$ (depending on the number of open/close cycles). During the frequency support operation, this behaviour will not be observed since the valve is opened once and continues to stay almost with the same opening throughout the whole synchronisation and generation cycle.

Overall, this mentioned temperature increase also depends on the fluid in the primary hydraulic circuit, since their thermoconductivities are different. This is one of the reasons why the proposed fluid change to a water-glycol solution should be analysed from the perspective mentioned here.

\subsection{Chapter Summary}

This chapter presents the final step in the synchronisation approach verification. Since it is required to achieve the highest level of precision of the NI controller's operation, it should be calibrated along with its analogue output module (NI9263). It was performed with the use of the standard calibration procedure provided by National Instruments.

The central PI-controller in the system should was tuned in order to reach stable synchronisation of the generator with the power grid. This process is presented in the chapter along with the sensitivity analysis for the feedfor- 
ward controller subsystem. The most suitable controller parameters had been verified through the use of the mathematical model and then were retuned accordingly to the experimental dynamic outputs.

The chapter depicts the experimental testing of the novel 2-stage synchronisation approach at slow acceleration, achieving synchronisation within $4 \mathrm{~s}$ after the system is triggered. The slow acceleration scenario was chosen for the tests to have more control over the system and have the option to stop the drivetrain acceleration manually. The same slow acceleration scenario was modelled in order to compare the simulated results with the experimental data. The comparison of the position, velocity, and phase difference profiles shows that the mathematical model was designed and characterised with a high level of precision.

Since the model is proven to be accurate and reliable, the fast synchronisation scenario was tested through the use of the model, not the experimental setup. This testing showed that the system is capable of synchronising the generator with a power grid in less than $1 \mathrm{~s}$ (the main requirement for FIR systems). Since the system was designed to provide frequency support in power grids, it is also important to verify its behaviour at different frequency droop rates. This has been done with the use of the model along with the robustness analysis. The chapter is finalised with the study on the influence of the hydraulic fluid temperature on the dynamics of the drivetrain. 


\section{Chapter 8}

\section{Conclusions}

The primary goal of this project was to develop a rapidly accelerated synchronous generation unit operating as an energy storage system. This was achieved through the use of a new acceleration and synchronisation approach for the synchronous generator. In the thesis, the focus was on the mathematical simulation and hardware implementation of the proposed synchronisation method. This was achieved through the use of a unique mathematical model and a ubiquitous set of sensors, actuators, and a high-speed industrial controller.

The research presented in the literature surrounding energy storage systems is primarily focused on the systems that can be used in combination with PV systems for household use, for instance for peak power reduction. Grid frequency stabilisation is typically presented in the literature by discussing Spinning Reserve systems, which are drastically different from Energy Storage systems based on their operating principles. When it comes to the use of EES systems in the power grids, the primary focus shifts towards mega CAES projects, whereas ss-CAES are more feasible and versatile. The research presented here can bridge these two mainly interconnected fields with each other and with the ss-CAES system for frequency support.

The designed simulation model helped to establish the stability boundaries of the system and also to verify the synchronisation approach. It led to the derivation of the parameter values of the controller that were later used in the hardware controller setup. By and large, the model can simulate the regimes of the system operation (fast acceleration within $1 \mathrm{~s}$ ) that are too dangerous to test experimentally.

The original ballistic synchronisation approach has been modified throughout the project and finally became a feasible synchronisation option (via the extended synchronisation windows). It is achieved with the use of extra proportional control loops for tracking the position and velocity measurements 
of the generator. Since the research into the use of unorthodox synchronisation methods is limited in the literature, the current research creates a strong knowledge base for other approaches in this field.

Hardware wise the current research proves the feasibility of hydraulic drivetrains for the rapid acceleration of synchronous machines (electrical machines in general) to allow their synchronisation with the power grid. Remembering the scale of the system, the research in this area is minimal and mainly focused on smaller technical solutions $(<10 \mathrm{~kW})$. Moreover, the studied system proved that a proportional valve could be accurate enough (in comparison to a servo valve) to control the hydraulic drivetrain to achieve its synchronisation with the electric network.

To achieve the ambitious task of utilising the proposed ss-CAES as an EES for frequency support, three objectives were set; The first, to verify the proposed improved ballistic synchronisation approach with the use of simulation tools. The second, to experimentally characterise the main components of the hydraulic circuit to make the mathematical model more realistic. It helps to tune the central control system in the way that this knowledge can be employed in the hardware setup. Lastly, to experimentally verify the proposed synchronisation approach with the use of the experimental build. A summary of these accomplishments is as follows:

1. Improved ballistic synchronisation approach. The initially designed ballistic approach was not able to provide enough time to connect a generator to a power grid (with the synchronisation windows up to 5-8 ms). Whereas, the current research shows that it is possible to employ the presented modified ballistic approach to achieve possible synchronisation conditions for more extended periods, up to at least 500-800 ms. This time is sufficient for any type of circuit breakers to connect a synchronous generator to an electric network.

This contribution has been published in Efim Sturov, Chris W. Bumby, Ramesh Rayudu, Rodney A. Badcock and Alan R. Wood, "Demonstrated rapid ballistic synchronisation from rest of a hydraulically-driven synchronous generator," IEEE Access, DOI:10.1109 / ACCESS.2018.2883110

2. Analysis of control valves (as an example - the proportional throttle valve TDA016). There are studies and manuals on the valve's parameterisation that are based on their manufacturer's data. However, there is no evidence of in-depth experimental analysis and verification of proportional throttle valves. The current research presents a unique hardware setup for the experimental characterisation of control valves. New knowledge 
on the valve's dynamics has been gathered with the use of the DAQ system and the valve's mathematical model. The collected experimental data showed that the traditional approach with the use of a second order transfer function is inadequate in some hydraulic setups. Hence, a 3-pole transfer function should be utilised for the modelling of valve dynamics.

This contribution is going to be published in Efim Sturov, Ramesh Rayudu and Chris W. Bumby, Modelling and experimental validation of an electrohydraulic drivetrain for synchronous generators in power engineering, ISA Transactions (under review).

3. Novel approach for the verification of the combined inertia of hydraulic drivetrains. No literature sources are showing the process of the inertia verification of complex hydraulic (electro-hydraulic) drivetrains. The presented study shows how low-resolution data from the installed encoder can be utilised for the accurate and feasible measurement of the train's inertia.

This contribution has been published in Efim Sturov, Chris W. Bumby, Ramesh Rayudu and Rodney A. Badcock, "Performance and stability analysis of hydraulically driven synchronous generators in power engineering," 2018 IEEE International Conference on Power System Technology (POWERCON), Guanzhou, China, 2018. doi: 10.1109/POWERCON.2018.8602006.

4. Fully studied hysteresis effect (experimentally verified) in the proportional valve and its influence on the electro-hydraulic drivetrain's dynamics. Contribution towards a newly designed hysteresis compensation algorithm in order to track a specified velocity of the motor.

This contribution has been published in Efim Sturov, Chris W. Bumby, Ramesh Rayudu and Rodney A. Badcock, "Rapid synchronisation procedure for a pneumohydraulically driven synchronous generator," 2017 IEEE Innovative Smart Grid Technologies - Asia (ISGT-Asia), Auckland, New Zealand, 2017. doi: 10.1109/ISGT-Asia.2017.8378373.

5. The above described experimental characterisation contributes towards an accurate mathematical model of the electro-hydraulic drivetrain. The presented research depicts a fully experimentally parameterised mathematical model simulating the ss-CAES system employed for rapid acceleration of synchronous generation units. The valve's model is combined 
with the experimentally verified motor's dynamics and the overall inertia of the hydraulic drivetrain. The drivetrain of the prototype has been verified with the use of the built DAQ system, ensuring its safe operation under different conditions and the synchronisation testing.

This contribution has been published in Efim Sturov, Chris W. Bumby, Ramesh Rayudu and Rodney A. Badcock, "Influence of fluid selection on synchronous generators power output in compressed air energy storage systems," 2017 IEEE Innovative Smart Grid Technologies - Asia (ISGTAsia), Auckland, New Zealand, 2017. doi: 10.1109/ISGT-Asia.2017.8378471

6. Implementation in hardware of the improved ballistic synchronisation approach with the use of high-speed industrial controllers. The central control system was developed around a National Instruments controller with the use of multiple toolkits and libraries to fully replicate the mathematical model of the controller and the synchronisation algorithms.

This contribution has been published in Efim Sturov, Ramesh Rayudu, Rodney A. Badcock, Yi Yu (Patrick) Chen, Alan R. Wood and Chris W. Bumby, "Rapid synchronization procedure for a synchronous generator employing ballistic trajectory control," 2016 IEEE Innovative Smart Grid Technologies - Asia (ISGT-Asia), Melbourne, VIC, 2016. doi:10.1109/ISGTAsia.2016.7796438.

\subsection{Future Work}

During the presented research, some questions have arisen that can be explored in the future. These are as follows:

- Programming of the Basler excitation system utilised in the prototype for energising the excitation windings of the synchronous generator. This results in generating the output voltage on the stator side of the electrical machine. It helps to fulfil all synchronisation requirements, making it possible to test the built-in hardware connection to a real power grid.

- Implementation of the proposed synchronisation approach by programming in the FPGA environment. The implementation of the DAQ algorithms and the primary synchronisation control strategy in the scan mode in Labview resulted in the increased computational burden on the controller. The latter mode executes codes at the frequency of $1 \mathrm{kHz}$, with some options to increase it up to $1 \mathrm{MHz}$. Since the main acceleration algorithm was coded in the Mathscript environment in Labview, the 
only option for running the controller loop was at $1 \mathrm{kHz}$, resulting in the sample rate of $1 \mathrm{~ms}$. The use of this algorithm also caused the increased computational burden on the controller causing jitters during the trajectory's calculations.

The FPGA mode enables the use of the FPGA chip in the controller running at $40 \mathrm{MHz}$, resulting in the sample rate of $25 \mathrm{~ns}$. The use of this mode also decreases the computational burden, making it possible to avoid jitters during the controller operation. This, in turn, makes the operation of the system more stable and accurate.

- The experimental testing of the proposed synchronisation approach in connection to a real electric network. The actual connection of the ssCAES to the power grid is complicated and can only be done after the fulfilment of many requirements imposed by the system operator (Transpower) and the IEEE and IEC standards and regulations. Since it is technically a commissioning process of a final product, the prototype should undertake all required tests and inspections, before it can be connected to the real grid. Taking into account that it is an unconventional generation unit, the inspection process can (and probably will) take much time, making the testing of the system impossible for other purposes. On top of this, the system is subject to exemptions from the standard testing procedures, making the commissioning process even more complicated. To synchronise the system with the power grid, the controller should be re-programmed. The described in Section 3 Phase Locked Loop system has to be implemented in the controller's code. Taking into account the related costs for the commissioning tests, as well as time constraints, the decision was made not to proceed with this testing.

- Measuring the temperature profile of the main hydraulic components of the hydraulic circuit. This knowledge can help to understand how the viscosity of the hydraulic fluid changes in real time and how it affects the system outputs. Due to the limitation on the number of analogue input modules on the cRIO board, it was not possible to achieve the simultaneous measurement of the pressure and temperature in the hydraulic circuit.

- Safety standards of the prototype should be improved to make it safer for its operators. The main observations, along with the proposed solutions are given in the next subsection "Health and safety concerns and improvements". 


\subsubsection{Health and safety concerns and improvements}

In order to improve the overall safety of the current prototype, some proposals have been put forward as follows.

A standard mobile pressure test bay should have full-width $6 \mathrm{~mm}$ doubleleaf swinging steel doors at one end (side) - they are designed to avoid fragment penetration. The doors should be equipped with a magnetic reed switch that will be interlocked with the pump used to pressurise the system. The roof design calls for a $6 \mathrm{~mm}$ thick steel plate roof.

The optional control room/office may be of conventional construction. Protection is provided by the bay walls, and there is no direct connection between the control room and the bay. Although the use of pan and zoom cameras (minimum two) is recommended, a viewing port may be provided between the control room and the bay. It should be a 3-inch thick piece of Lexan (or equivalent such as UL-5 or NIJ-III rated glazing $38 \mathrm{~mm}$ thick) to provide fragment protection.

Safety Interlocks - Safety Interlocks should be provided in the design to ensure that personnel are not exposed to the potential hazards associated with the pressurised system. Specifically:

- The door latching mechanism should be interlocked with pump pressure sensors to ensure that the doors to the test bay cannot be opened (without an alarm) when the system is pressurised, or the pump is running.

- The power to the pump should be turned off when a door is open. The warning light on the outside of the test bay will be activated whenever the system is pressurised.

- The schematic logic is shown in Table 8.1

Cameras - Cameras located within the bay could be wall mounted near the ceiling but should be below any overhead crane location. A minimum of two cameras (four are recommended - one in each corner) with pan and zoom features are required. The cameras are intended to provide the operators with a view of the test and are not used for detailed data recording; therefore conventional closed circuit television (CCTV) cameras and monitors are adequate. The cameras are protected from dirt, moisture, and debris by plastic covers. The covers are not intended to protect the cameras from fragments.

It should be noted that a failure of the electro-hydraulic drivetrain will have a significant impact on the cell design. Taking into account that a failure during pneumatic tests will result in both blast and fragment loadings on the cell 
Table 8.1: Door control schematic

\begin{tabular}{|l|l|l|l|l|}
\hline Door Position & Pressure sensor & Warning Lights & Alarm & Pressure Cutoff \\
\hline Closed & Off & Off & Off & Inactive \\
\hline Open & Off & Off & Off & Inactive \\
\hline Closed & On & On & Off & Inactive \\
\hline Open & On & Blinking & On & Activated \\
\hline
\end{tabular}

structure, while a failure during a hydrostatic test would only result in fragment loadings.

\section{Potential Fragments and Perforation Protection}

The fragments that may result from a tool failure may be categorised as either on-axis or off-axis. Off-axis fragments are typically smaller, are somewhat random in their orientation, and may have higher velocities than on-axis fragments. On-axis fragments are typically plugs, end caps, or other fittings. The velocity of on-axis fragments is highly dependent on the engagement length of the threads holding the plug or cap in place. The higher the engagement length, the higher the potential fragment velocity will be since the fragment is essentially "pushed" by the expanding fluid for a more extended period. BakerRisk conducted a series of tests in 2006 on behalf of the High-Pressure Testing Safety Joint Industry Program to determine potential fragment velocities as a function of pressure. These tests are representative of some of the offand on-axis fragments that may result from a pressurised tool failure. The tests consisted of over-pressurising a fitting connection so that the fitting would be "fired" at a steel plate and other various targets. High-speed cameras measured the fitting velocities. A $6 \mathrm{~mm}$ thick steel plate was effective in preventing perforation by the off-axis fittings tested at pressures at or below 22,500 psi (1550 bar). 


\section{Bibliography}

[1] I. N. Moghaddam and B. Chowdhury, "Battery energy storage sizing with respect to pv-induced power ramping concerns in distribution networks," in Power \& Energy Society General Meeting, pp. 1-5, July 2017.

[2] C. Klabunde, N. Moskalenko, P. Lombardi, P. Komarnicki, and Z. Styczynski, "Optimal onshore wind power integration supported by local energy storages," in Power \& Energy Society General Meeting, pp. 1-5, July 2015.

[3] J. Feng, B. Zeng, D. Zhao, G. Wu, Z. Liu, and J. Zhang, "Evaluating demand response impacts on capacity credit of renewable distributed generation in smart distribution systems," IEEE Access, vol. 6, pp. 1430714317, September 2018.

[4] Y. Jiang, G. Wang, S. Roy, and C.-C. Liu, "Power system severe contingency screening considering renewable energy," in Power and Energy Society General Meeting (PESGM), 2016, pp. 1-5, November 2016.

[5] System Operator, "Under frequency event report," Tech. Rep. , Transpower New Zealand Limited, 2016.

[6] E. Serban, M. Ordonez, and C. Pondiche, "Voltage and frequency grid support strategies beyond standards," IEEE Transactions on Power Electronics, vol. 32, pp. 298-309, March 2017.

[7] System Operator, "Asset owner obligations (aopo)," Tech. Rep. , Transpower New Zealand Limited, 2011.

[8] A. Susperregui, J. Jugo, I. Lizarraga, and G. Tapia, "Automated control of doubly fed induction generator integrating sensorless parameter estimation and grid synchronisation," IET Renewable Power Generation, vol. 8, pp. 76-89, January 2014.

[9] AEA, "Energy storage and management study," Tech. Rep. , Scottish Government ELL/000/077, 2010. 
[10] B. Dunn, H. Kamath, and J.-M. Tarascon, "Electrical Energy Storage for the Grid: A Battery of Choices," Science, vol. 334, pp. 928-935, November 2011.

[11] Electricity Commission, "Current arrangements for frequency , voltage , reliability and security," Tech. Rep. , EC FK, 2007.

[12] L. Langston, G. Opdyke, and E. Dykewood, "Introduction to Gas Turbines for Non-engineers," Global Gas Turbine News, vol. 37, no. 2, 1997.

[13] D. Rastler, “Electricity Energy Storage Technology Options,” Tech. Rep. , Electric Power Research Institute, Palo Alto, 2010.

[14] J. Eyer and G. Corey, "Energy Storage for the Electricity Grid : Benefits and Market Potential Assessment Guide," Tech. Rep. , Sandia National Laboratories, Albuquerque, 2010.

[15] J. Baker, "New technology and possible advances in energy storage," Energy Policy, vol. 36, pp. 4368-4373, December 2008.

[16] P. J. Hall and E. J. Bain, "Energy-storage technologies and electricity generation," Energy Policy, vol. 36, pp. 4352-4355, December 2008.

[17] Anon, "Fire Resistant Hydraulic Fluids," Lubrication, vol. 48, no. 11, pp. 161-180, 1962.

[18] C. Cargo, A. Hillis, and A. Plummer, "Strategies for active tuning of wave energy converter hydraulic power take-off mechanisms," Renewable Energy, vol. 94, pp. 32-47, August 2016.

[19] P. Y. Li and M. Saadat, "An approach to reduce the flow requirement for a liquid piston near-isothermal air compressor / expander in a compressed air energy storage system," IET Renewable Power Generation, vol. 10, pp. 1506-1514, November 2016.

[20] Y.-Y. P. Chen, “Rapid Synchronization Control of Compressed Gas Energy Storage Generator During Power Grid Contingent and Extended Contingent Events," M.S. thesis, University of Canterbury, 2015.

[21] Y. Y. Chen (Patrick), A. R. Wood, C. W. Bumby, and R. A. Badcock, "Rapid synchronisation of fast instantaneous reserves CAES generator," International Journal of Electrical Power and Energy Systems, vol. 94, pp. 203-212, January 2018. 
[22] "EHL Group." http://www.ehltd.co.nz, Online; accessed July 2019.

[23] S. Westphalia, A. Sciences, D. Soest, and L. Ring, "Challenges in Integrating Distributed Energy Storage Systems into Future Smart Grid," pp. 1627-1632, November 2008.

[24] A. Akele-sita and S. Chowdhury, "Optimal allocation of energy storage in a future congolese power system," in 50th International Universities Power Engineering Conference (UPEC), pp. 1-6, December 2015.

[25] T. R. Ayodele and A. S. O. Ogunjuyigbe, "Mitigation of wind power intermittency: Storage technology approach," Renewable and Sustainable Energy Reviews, vol. 44, pp. 447-456, April 2015.

[26] A. Etxeberria, I. Vechiu, J. Vinassa, and H. Camblong, "Hybrid Energy Storage Systems for Renewable Energy Sources Integration in Microgrids : A Review," IPEC, 2010 Conference Proceedings, pp. 532-537, January 2010.

[27] H. Ibrahim, K. Belmokhtar, and M. Ghandour, "Investigation of usage of compressed air energy storage for power generation system improving - Application in a microgrid integrating wind energy," Energy Procedia, vol. 73, pp. 305-316, June 2015.

[28] The International Renewable Energy Agency, "Renewables and electricity storage a technology roadmap for remap 2030," Tech. Rep. May, 2015.

[29] S. Vazquez, S. Lukic, E. Galvan, L. G. Franquelo, J. M. Carrasco, and J. I. Leon, "Recent advances on Energy Storage Systems," in IECON 2011 37th Annual Conference of the IEEE Industrial Electronics Society, pp. 46364640, June 2011.

[30] F. Díaz-González, A. Sumper, O. Gomis-Bellmunt, and R. VillafáfilaRobles, "A review of energy storage technologies for wind power applications," Renewable and Sustainable Energy Reviews, vol. 16, pp. 2154-2171, May 2012.

[31] I. Hadjipaschalis, A. Poullikkas, and V. Efthimiou, "Overview of current and future energy storage technologies for electric power applications," Renewable and Sustainable Energy Reviews, vol. 13, pp. 1513-1522, August - September 2009. 
[32] T. Christen and M. W. Carlen, "Theory of Ragone plots," Journal of Power Sources, vol. 91, pp. 210-216, December 2000.

[33] D. O. Akinyele and R. K. Rayudu, "Review of energy storage technologies for sustainable power networks," Sustainable Energy Technologies and Assessments, vol. 8, pp. 74-91, December 2014.

[34] H. Chen, T. Ngoc, W. Yang, C. Tan, and Y. Li, "Progress in electrical energy storage system : A critical review," Progress in Natural Science, vol. 19, pp. 291-312, March 2009.

[35] D. Pavković, M. Hoić, J. Deur, and J. Petrić, “Energy storage systems sizing study for a high-altitude wind energy application," Energy, vol. 76, pp. 91-103, November 2014.

[36] H. Ibrahim, A. Ilinca, and J. Perron, "Energy storage systems. Characteristics and comparisons," Renewable and Sustainable Energy Reviews, vol. 12, pp. 1221-1250, June 2008.

[37] S. Vazquez, S. M. Lukic, E. Galvan, L. G. Franquelo, and J. M. Carrasco, “Energy Storage Systems for Transport and Grid Applications," IEEE Transactions on Industrial Electronics, vol. 57, pp. 3881-3895, September 2010.

[38] S. Lemofouet and A. Rufer, "Hybrid energy storage systems based on compressed air and supercapacitors with maximum efficiency point tracking," in 2005 European Conference on Power Electronics and Applications, pp. 10 pp.-P.10, July 2005.

[39] A. Rufer and S. Lemofouet, "Efficiency considerations and measurements of a hybrid energy storage system based on compressed air and super capacitors," EPE-PEMC 2006: 12th International Power Electronics and Motion Control Conference, Proceedings, pp. 2077-2081, June 2007.

[40] G. Wang, M. Ciobotaru, and V. G. Agelidis, "Power Smoothing of Large Solar PV Plant Using Hybrid Energy Storage," Sustainable Energy, IEEE Transactions on, vol. 5, pp. 834-842, March 2014.

[41] M. Bhaskarnaik, P. Kumar, and S. Majhi, "Analysis of city-Capabus transportation system failures and solutions with FLC," IEEE Conference on Intelligent Transportation Systems, Proceedings, ITSC, pp. 1900-1905, January 2013. 
[42] Y. Ye, R. Sharma, and P. Garg, "An integrated power management strategy of hybrid energy storage for renewable application," Proceedings, IECON 2014 - 40th Annual Conference of the IEEE Industrial Electronics Society, pp. 3088-3093, February 2015.

[43] Y. M. Kim, D. G. Shin, S. Y. Lee, and D. Favrat, "Isothermal transcritical $\mathrm{CO} 2$ cycles with TES (thermal energy storage) for electricity storage," Energy, vol. 49, pp. 484-501, January 2013.

[44] H. Sun, X. Luo, and J. Wang, "Simulation study of energy efficiency for a hybrid wind turbine system," Industrial Technology (ICIT), 2013 IEEE International Conference, pp. 781-786, April 2013.

[45] H. Sedighnejad, T. Iqbal, and J. Quaicoe, "Performance evaluation of a hybrid wind-diesel-compressed air energy storage system," in 2011 24th Canadian Conference on Electrical and Computer Engineering(CCECE), pp. 000270-000273, September 2011.

[46] M. Budt, D. Wolf, R. Span, and J. Yan, "A review on compressed air energy storage: Basic principles, past milestones and recent developments," Applied Energy, vol. 170, pp. 250-268, May 2016.

[47] G. B. Liu, Q. C. Yang, Z. G. Qian, Y. Y. Zhao, L. Wang, L. S. Li, and B. Tang, "Study on Release Process of Micro-CAES Used Screw Expander," Applied Mechanics and Materials, vol. 457-458, pp. 379-383, October 2013.

[48] J. Kim, E. Yim, C. Jeon, C. Jung, and B. Han, "Cold performance of various biodiesel fuel blends at low temperature," International Journal of Automotive Technology, vol. 13, no. 2, pp. 293-300, 2012.

[49] V. Kokaew, S. M. Sharkh, M. Moshrefi-Torbati, V. Kokaew, and M. Moshrefi-Torbati, "Maximum Power Point Tracking of a Small-Scale Compressed Air Energy Storage System Step size in a coarse-tuning area. $\Delta \omega \mathrm{f}$ Step size in a fine-tuning area," IEEE Transactions on Industrial Electronics, vol. 63, pp. 985-994, September 2016.

[50] J. J. Proczka, K. Muralidharan, D. Villela, J. H. Simmons, and G. Frantziskonis, "Guidelines for the pressure and efficient sizing of pressure vessels for compressed air energy storage," Energy Conversion and Management, vol. 65, pp. 597-605, January 2013.

[51] B. Elmegaard and W. Brix, "Efficiency of Compressed Air Energy Storage," The 24th International Conference on Efficiency, Cost, Optimization, 
Simulation and Environmental Impact of Energy Systems, pp. 1-12, July 2011.

[52] Tian Xia, Lei He, Ning An, Muyi Li, and Xin Li, “Electromechanical transient modeling research of energy storage system based on power system security and stability analysis," 2014 International Conference on Power System Technology (POWERCON 2014), pp. 20-22, December 2014.

[53] H. Guo, Y. Xu, H. Chen, and X. Zhou, "Thermodynamic characteristics of a novel supercritical compressed air energy storage system," Energy Conversion and Management, vol. 115, pp. 167-177, May 2016.

[54] T. L. Brown, V. P. Atluri, and J. P. Schmiedeler, "A low-cost hybrid drivetrain concept based on compressed air energy storage," Applied Energy, vol. 134, pp. 477-489, December 2014.

[55] J. Regehr and U. Duongsaa, "Deriving abstract transfer functions for analyzing embedded software," ACM SIGPLAN Notices, vol. 41, p. 34, July 2006.

[56] B. Zhang, "Modeling and Analysis of Hydraulic Energy Storage System for Hybrid Locomotives," M.S. thesis, The University of Toledo, 2010.

[57] S. S. Raza, I. Janajreh, and C. Ghenai, “Sustainability index approach as a selection criteria for energy storage system of an intermittent renewable energy source," Applied Energy, vol. 136, pp. 909-920, December 2014.

[58] L. Wang, H. Cheung, A. Hamlyn, and R. Cheung, "Model prediction adaptive control of inter-area oscillations in multi-generators power systems," 2009 IEEE Power and Energy Society General Meeting, PES '09, pp. 1-7, October 2009.

[59] N. A. Belyaev, Y. V. Khrushchev, S. V. Svechkarev, A. V. Prokhorov, and L. Wang, "Generator to grid adaptive synchronization technique based on reference model," 2015 IEEE Eindhoven PowerTech, September 2015.

[60] F. U. Yongling, Q. I. Haitao, L. U. Yueliang, G. U. O. Rongsheng, L. I. Zhufeng, and X. U. E. Jing, "A Novel Electrical Servo Variable Displacement Hydraulic Pump Used for Integrated Actruator in MEA," in 28th International Congress of the Aeronautical Sciences, (Brisbane), pp. 1-6, 2012. 
[61] I. Cristian, "An Analysis of the Dynamic Behaviour of the Linear Hydraulic Motor - Non-linear / Liniarized Cutting Force System," RECENT, vol. 1, no. 11, pp. 188-191, 2010.

[62] A. Abdul and M. Hassan, "Design and Analysis of Electro-Hydraulic Servo System for Speed Control of Hydraulic Motor," Journal of Engineering, vol. 19, no. 5, pp. 562-573, 2013.

[63] K. Sailan, K. D. Kuhnert, and S. Sadege, "Development of ElectroHydraulic Servo Drive Train System for DORIS Robot," in Scientific Cooperations International Workshops on Electrical and Computer Engineering Subfields, (Istanbul), pp. 212-218, December 2014.

[64] A. A. Aly, "Modeling and Control of an Electro-Hydraulic Servo Motor Applying Velocity Feedback Control Strategy," in International Mechanical Engineering Conference, (Kuwait), pp. 334-342, December 2004.

[65] S. Madanipour, "Modeling and Control of a Servo Hydraulic Motor," Indian J.Sci.Res., vol. 1, no. 2, pp. 770-774, 2014.

[66] F. Lin, "The Design and Simulation of Electro-Hydraulic Velocity Control System," IFIP Advances in Information and Communication Technology, vol. 347, pp. 568-574, 2011.

[67] MOOG, "Transfer Functions for MOOG Servovalves," Tech. Rep, Aug. 1965.

[68] E. Toraman, "Modeling, simulation and experimental verification of hydraulic fan drive system," M.S. thesis, Middle East Technical University, 2013.

[69] Lauvli, Per-Willy, "Modeling, Simulation and Experimentation of a Hydrostatic Transmission," M.S. thesis, University of Agder, 2010.

[70] Mathworks, "Simscape TM Fluids TM User 's Guide." http://au.mathworks.com/help/pdfdoc/physmod/hydro/hydroug.pdf, 2016. Online; accessed August 2019.

[71] J. Watton, Modelling, Monioring \& Diagnostic Techniques for Fluid Power Systems. Cardiff: Springer, 2007. ISBN 978-0874216561.

[72] P. Puddu and M. Paderi, "Hydro-pneumatic accumulators for vehicles kinetic energy storage: Influence of gas compressibility and thermal losses on storage capability," Energy, vol. 57, pp. 326-335, August 2013. 
[73] V. S. Tenali, "Simulation of Electro-Hydraulic Servo Actuator," M.S. thesis, National Institute of Technology, 2007.

[74] D. Maneetham and N. Afzulpurkar, "Modeling, simulation and control of high speed nonlinear hydraulic servo system," World Journal of Modelling and Simulation, vol. 6, no. 1, pp. 27-39, 2010.

[75] A. Dell'Amico and P. Krus, "Modelling and experimental validation of a nonlinear proportional solenoid pressure control valve," International Journal of Fluid Power, vol. 17, pp. 90-101, February 2016.

[76] Y. Zhu and B. Jin, "Analysis and modeling of a proportional directional valve with nonlinear solenoid," Journal of the Brazilian Society of Mechanical Sciences and Engineering, vol. 38, no. 2, pp. 507-514, 2016.

[77] A. Milecki and D. Rybarczyk, "Modelling of an electrohydraulic proportional valve with a synchronous motor," 2015. ISSN 00392480.

[78] V. Tchkalov and S. Miller, "Parameterization of Directional and proportional valves in Simhydraulics." http://www.mathworks.com/SimHydraulics.pdf, $2014 . \quad$ online; accessed August 2019.

[79] A. Barakat, S. Tnani, G. Champenois, and E. Mouni, "Analysis of synchronous machine modeling for simulation and industrial applications," Simulation Modelling Practice and Theory, vol. 18, pp. 1382-1396, October 2010.

[80] R. Amirante, P. G. Moscatelli, and L. A. Catalano, "Evaluation of the flow forces on a direct (single stage) proportional valve by means of a computational fluid dynamic analysis," Energy Conversion and Management, vol. 48, pp. 942-953, March 2007.

[81] F. Norvelle, Elecrohydraulic Control Systems. New Jersey: Prentice Hall, 2000. ISBN 978=0137163595.

[82] H. Machinery, "Dynamic model of the rotative hydraulic motor under constant pressure," Simulation, pp. 1-5, October 2004.

[83] R. H. Park, "Two-reaction theory of synchronous machines generalized method of analysis-part I," Transactions of the American Institute of Electrical Engineers, vol. 48, pp. 716-727, July 1929. 
[84] R. H. Park, "Two Reaction Theory of Synchronous Machines Generalized Method of Analysis-Part II," in Winter Convention of A.I.E.E., (New Y), pp. 352-354, July 1933.

[85] F. Demello and C. Concordia, "Concepts of Synchronous Machine Stability as Affected by Excitation Control," IEEE Transactions on Power Apparatus and Systems, vol. PAS-88, pp. 316-329, April 1969.

[86] M. Saidy, "Block diagram transfer function model of a generator including damper windings," IEE Proceedings - Generation, Transmission and Distribution, vol. 141, p. 599, February 1994.

[87] M. Saidy and F. M. Hughes, "An extended block diagram transfer function model of a synchronous machine," Science, vol. 18, pp. 139-142, February 1996.

[88] P. Dandeno, P. Kundur, S. Umans, I. Kamwa, H. Karmaker, S. Salon, M. Shah, and A. El-Serafi, "IEEE Guide for Synchronous Generator Modeling Practices and Applications in Power System Stability Analyses," 2003.

[89] D. C. Aliprantis, S. D. Sudhoff, S. Member, and B. T. Kuhn, "A synchronous machine model with saturation and arbitrary rotor network representation," Energy, vol. 20, pp. 584-594, August 2005.

[90] P. Aree, "Investigation of local load effect on damping characteristics of synchronous generator using transfer-function block-diagram model," Songklanakarin J. Sci. Technol., vol. 27, pp. 827-838, November 2005.

[91] Y. Najafi Sarem, J. Poshtan, M. Ghomi, and M. Poshtan, "Synchronous generator parameters estimation," in 2007 International Conference on Intelligent and Advanced Systems, pp. 870-875, IEEE, October 2007.

[92] D.-P. Molenaar, O. H. Bosgra, and M. J. Hoeijmakers, "Time-Domain Identification of Synchronous Generator Transfer Functions," Journal of Solar Energy Engineering, vol. 124, p. 419, November 2002.

[93] A. B. Chowdhury, A. Kulhare, and G. Raina, "A study of the SMIB power system model with delayed feedback," 2011 International Conference on Power and Energy Systems, ICPS 2011, pp. 1-6, February 2011.

[94] S. Kalyani, M. Prakash, and G. A. Ezhilarasi, "Transient stability studies in SMIB system with detailed machine models," 2011 International 
Conference on Recent Advancements in Electrical, Electronics and Control Engineering, IConRAEeCE'11 - Proceedings, pp. 459-464, January 2011.

[95] M. Ladjavardi, M. A. S. Masoum, and S. M. Islam, "Impact of SG damper windings on small-signal stability of A distorted SMIB including time and space harmonics," 40th North American Power Symposium, NAPS2008, pp. 1-7, November 2008.

[96] T. Roy, M. Mahmud, W. Shen, and A. Oo, “Nonlinear excitation control of synchronous generators based on adaptive backstepping method," Proceedings of the 2015 10th IEEE Conference on Industrial Electronics and Applications, ICIEA 2015, pp. 11-16, November 2015.

[97] J. Morsali, A. Parhizkar, H. Morsali, and A. Zeinali, "Recommendation of equipping steam unit of khoy combined cycle power plant with novel PID-PSS2B industrial stabilizer to decrease risk of sudden trips," 2014 5th Conference on Thermal Power Plants, CTPP 2014, pp. 89-94, February 2015.

[98] T. Hammons, "Erratum: Comparisons of synchronous-machine models in the study of the transient behaviour of electrical power systems," Proceedings of the Institution of Electrical Engineers, vol. 119, p. 1337, September 1972.

[99] Y. Zimmerman, Y. Oshman, and A. Brandes, "Improving the accuracy of analog encoders via Kalman filtering," Control Engineering Practice, vol. 14, pp. 337-350, April 2006.

[100] S. Wang and S. Wan, "Velocity and acceleration computations by singledimensional Kalman filter with adaptive noise variance," Przeglad Elektrotechniczny, vol. 88, no. 2, pp. 283-287, 2012.

[101] M. Hilairet, F. Auger, and E. Berthelot, "Speed and rotor flux estimation of induction machines using a two-stage extended Kalman filter," Automatica, vol. 45, pp. 1819-1827, August 2009.

[102] W. Ames and C. Brezinski, Numerical recipes in Fortran (The art of scientific computing), vol. 35. 1993. ISBN 052143064X.

[103] Á. Valera, M. Vallés, L. Marín, and P. Albertos, "Design and implementation of Kalman filters applied to LEGO NXT based robots," IFAC Proceedings Volumes (IFAC-PapersOnline), vol. 18, pp. 9830-9835, January 2011. 
[104] P. Kundur, G. J. Rogers, D. Y. Wong, L. Wang, and M. G. Lauby, “A comprehensive computer program package for small signal stability analysis of power systems," Power Systems, IEEE Transactions on, vol. 5, pp. 10761083, November 1990.

[105] E. Sturov, C. W. Bumby, R. Rayudu, and R. A.Badcock, “Rapid synchronisation procedure for a pneumo-hydraulically driven synchronous generator," in 2017 IEEE Innovative Smart Grid Technologies - Asia (ISGT-Asia), (Auckland), pp. 1-5, IEEE, June 2017.

[106] P. Kundur, Power System Stability and Control. McGraw-Hill, 1994. ISBN 9780070359581.

[107] B. Dunn, H. Kamath, and J.-M. Tarascon, "Electrical Energy Storage for the Grid: A Battery of Choices," Science, vol. 334, pp. 928-935, November 2011.

[108] E. Sturov, Y.-Y. P. Chen, A. Wood, R. Rayudu, R. Badcock, and C. Bumby, "Rapid synchronization procedure for a synchronous generator employing ballistic trajectory control," in ISGT PES 2016 Asia, (Melbourne), December 2016.

[109] IEEE Standards Association and others, "IEEE 1547 Standard for Interconnecting Distributed Resources with Electric Power Systems," IEEE Standards Association, Piscataway, 2003.

[110] R. Amirante, A. Innone, and L. A. Catalano, "Boosted PWM open loop control of hydraulic proportional valves," Energy Conversion and Management, vol. 49, pp. 2225-2236, August 2008.

[111] W. D. Pilkey and D. F. Pilkey, Peterson's stress concentration factors. John Wiley \& Sons, 2008. ISBN 9780471538493.

[112] J. E. Shigley, Shigley's mechanical engineering design. Tata McGraw-Hill Education, 2011. ISBN 9780071311137.

[113] F. P. Beer, R. Johnston, J. Dewolf, and D. Mazurek, Mechanics of Materials. McGraw-Hill Boston, 2006. ISBN 0073398233.

[114] W. D. Pilkey and W. D. Pilkey, Formulas for stress, strain, and structural matrices. Wiley New York, 1994. ISBN 9780471527466.

[115] W. C. Young and R. G. Budynas, Roark's formulas for stress and strain, vol. 7. McGraw-Hill New York, 2002. ISBN 007072542X. 
[116] Texas Instruments, “Opa548 high-voltage, high-current operational amplifier," http: //www.ti.com/lit/ds/symlink/opa548.pdf, Online; accessed December 2018.

[117] Cashco, "Fluid Flow Basics of Throttling Valves." https://www . controlglobal.com/assets/RefBookCashco_Fluid.pdf, 2011. Online; accessed August 2019.

[118] N. S. Nise, CONTROL SYSTEMS ENGINEERING, (With CD). John Wiley \& Sons, 2007. ISBN 978-1118170519.

[119] H. W. Wu and C. B. Lee, "Influence of a relief valve on the performance of a pump/inverter controlled hydraulic motor system," Mechatronics, vol. 6, pp. 1-19, February 1996.

[120] B. R. Munson, T. H. Okiishi, W. W. Huebsch, and A. P. Rothmayer, Fundamentals of Fluid Mechanics. Wiley Singapore, 2013. ISBN 9780471469254.

[121] IEEE Standard 421.5-2005, IEEE Recommended Practice for Excitation System Models for Power System Stability Studies. New York: IEEE, 2005. ISBN 0738147869.

[122] E. Sturov, C. Bumby, R. Rayudu, and R. Badcock, "Rapid-start generators in an Energy Storage System: Influence of fluid selection on the components of a hydraulic drive-train," in EEA Conference and Exhibition, 21-23 June, 2017.

[123] E. Sturov, C. W.Bumby, R. Rayudu, and R. A.Badcock, "Influence of fluid selection on synchronous generators power output in compressed air energy storage systems," in 2017 IEEE Innovative Smart Grid Technologies - Asia (ISGT-Asia), (Auckland), pp. 1-6, IEEE, December 2017.

[124] Standards GOST, "Online calculators." https://planetcalc.com/ 2834/, 2003. Online; accessed August 2019.

[125] B. E. Rapp, Microfluidics: Modeling, Mechanics and Mathematics. William Andrew, 2016. ISBN 9781455731311. 